\title{
Trim Solutions of Multirotor Vehicles using A Fast Performance Prediction Method
}

\author{
by \\ Julia D. Tsaltas \\ Bachelor of Engineering, The University of Western Ontario (2015)
}

\author{
A thesis \\ presented to Ryerson University \\ in partial fulfilment of the \\ requirements for the degree of \\ Master of Applied Science \\ in the program of \\ Aerospace Engineering
}

Toronto, Ontario, Canada, 2018

(c) Julia D. Tsaltas, 2018 


\section{AUTHOR'S DECLARATION FOR ELECTRONIC SUBMISSION OF A THESIS}

I hereby declare that I am the sole author of this thesis. This is a true copy of the thesis, including any required final revisions, as accepted by my examiners.

I authorize Ryerson University to lend this thesis to other institutions or individuals for the purpose of scholarly research.

I further authorize Ryerson University to reproduce this thesis by photocopying or by other means, in total or in part, at the request of other institutions or individuals for the purpose of scholarly research.

I understand that my dissertation may be made electronically available to the public. 


\title{
Trim Solutions of Multirotor Vehicles using a Fast Performance Prediction Method Julia D. Tsaltas
}

Master of Applied Science, Aerospace Engineering, Ryerson University (2018)

\begin{abstract}
A fast multirotor performance prediction method is presented. The method uses an algorithm to determine the flight performance and trim solutions of multirotor vehicles in steady, level flight. The method considers parasitic drag, force trim, fuselage interference, rotor interference, moment trim, and power prediction. In order to validate the method, vehicle lift, drag, and pitching moment predictions are compared to experimental data from NASA Ames for the 3DR Solo, a commercially available vehicle. The performance comparison with wind tunnel data show similar lift, drag and pitching moment trends when using estimated rotor and vehicle geometries. In addition, the predicted rotor speeds, vehicle power, and vehicle pitch are compared to flight test data of the Aeryon SkyRanger. The lead and rear rotor speed results show that the application of moment trim into the performance model provides rotor speed estimates that reflect the differential rotor speeds the flight test. An orientation study is conducted to explore the effects of rotor and fuselage interference velocities on rotor performance and the performance differences of a four-rotor vehicle flying in diamond and square configurations. Finally, a mass offset study is presented to predict the changes in rotor speed distribution of a SkyRanger vehicle when a $100 \mathrm{~g}$ mass is added to the support arm, which simulates asymmetry in centre of gravity location. The predicted performance results show overlapping results with flight testing with and without the mass offset at airspeeds below $5 \mathrm{~m} / \mathrm{s}$. At higher airspeeds, the rotor speed predictions that are established by moment trim requirements reflect the rotor speed trends shown from flight test data.
\end{abstract}




\section{TABLE OF CONTENTS}

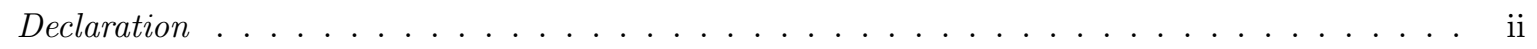

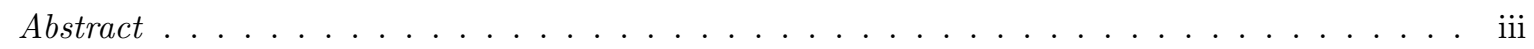

List of Tables . . . . . . . . . . . . . . . . . . . . . vii

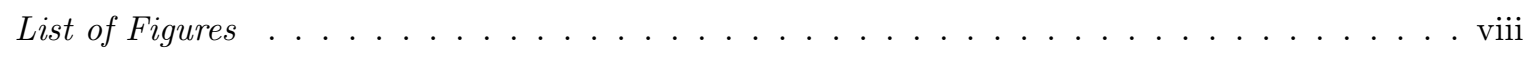

List of Symbols . . . . . . . . . . . . . . . . . . . . . . . . xi

\begin{tabular}{lll}
\hline & INTRODUCTION & 1
\end{tabular}

1.1 Small Multirotor Aerial Systems $\ldots \ldots \ldots \ldots \ldots \ldots \ldots$

2 MULTIROTOR AERODYNAMICS AND FLIGHT DYNAMICS $\quad 6$

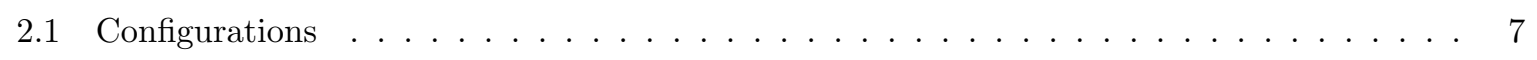

2.2 Roll, Pitch, and Yaw Control $\ldots \ldots \ldots \ldots \ldots \ldots \ldots$

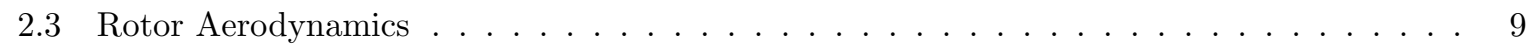

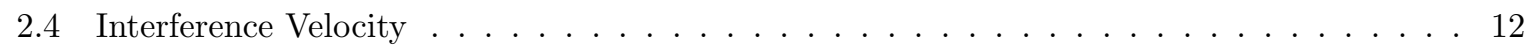

2.5 Stability, Control, and Trim $\ldots \ldots \ldots \ldots \ldots \ldots \ldots \ldots$

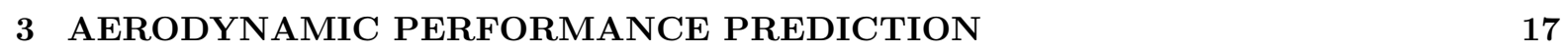

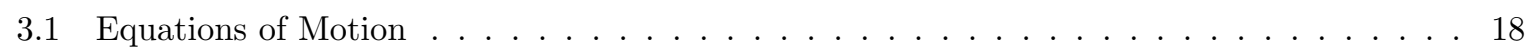

3.2 Drag and Lift Prediction $\ldots \ldots \ldots \ldots \ldots \ldots \ldots \ldots$

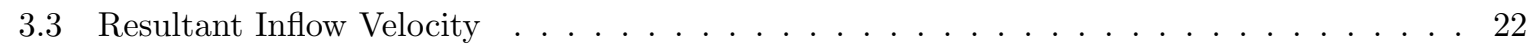

3.3 .1 Rotor Interference $\ldots \ldots \ldots \ldots \ldots \ldots \ldots$

3.3 .2 Wake Interference Model Limitations . . . . . . . . . . . . . . . . . . . . 26

3.3 .3 Fuselage Interference $\ldots \ldots \ldots \ldots \ldots \ldots$ 
3.4 Interference Model $\ldots \ldots \ldots \ldots \ldots \ldots$

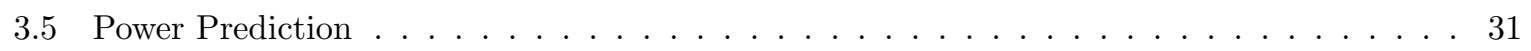

3.6 Moment Trim . . . . . . . . . . . . . . . . . . . . . . . . 32

3.7 Prediction Model Algorithm $\ldots \ldots \ldots \ldots$

4 COMPARISON OF PREDICTION AND EXPERIMENTAL WIND TUNNEL AND $\begin{array}{ll}\text { FLIGHT TEST RESULTS } & 37\end{array}$

4.13 DR Solo Performance $\ldots \ldots \ldots \ldots \ldots$

4.1 .1 Revised Prediction Method . . . . . . . . . . . . . . . . . . . . . . . . 38

$4.1 .2 \quad$ Rotor Lift and $\mathrm{Drag} \ldots \ldots \ldots \ldots \ldots \ldots \ldots$

$4.1 .3 \quad$ Vehicle Lift and $\operatorname{Drag} \ldots \ldots \ldots \ldots \ldots \ldots \ldots$

$4.1 .4 \quad$ Full Vehicle $\ldots \ldots \ldots \ldots$. . . . . . . . . . . . . . . . . . . . . . . . . . . . 43

$4.2 \quad$ Flight Test Results $\ldots \ldots \ldots \ldots \ldots \ldots \ldots \ldots \ldots \ldots \ldots$

\begin{tabular}{|lll}
\hline 5 & ORIENTATION STUDY & 48
\end{tabular}

5.1 Vehicle Performance $\ldots \ldots \ldots \ldots \ldots$

5.2 Interference Velocities $\ldots \ldots \ldots \ldots \ldots \ldots \ldots \ldots \ldots$

$5.2 .1 \quad$ Rotor Interference Velocity $\ldots \ldots \ldots \ldots \ldots \ldots$. . . . . . . . . . . . . 55

5.2 .2 Fuselage Interference $\ldots \ldots \ldots \ldots \ldots \ldots \ldots$

5.2 .3 Inflow Velocity and Inflow Angle $\ldots \ldots \ldots \ldots$. . . . . . . . . . . . 61

5.3 Moment Trim . . . . . . . . . . . . . . . . . . . . . . 65

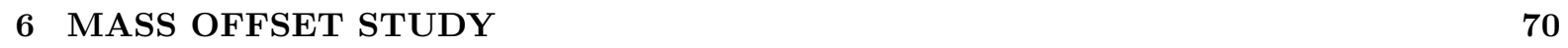

6.1 Performance Comparison with Flight Test Results $\ldots \ldots \ldots \ldots$. . . . . . . . . 70

\begin{tabular}{lll}
\hline 7 & CONCLUSIONS & 75
\end{tabular}

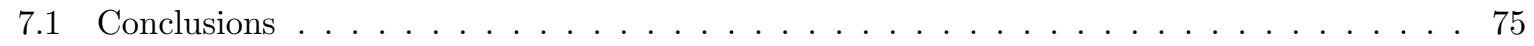

$7.2 \quad$ Future Development $\ldots \ldots \ldots \ldots \ldots \ldots \ldots \ldots$

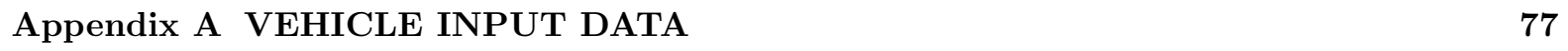

A.1 3DR Solo Input Parameters . . . . . . . . . . . . . . . . . . . . . . . 77

A.2 GRCSP 10x8 Rotor Geometry Input . . . . . . . . . . . . . . . . . . . 78 
A.3 SkyRanger Input Parameters $\ldots \ldots \ldots \ldots$

A.4 T-Motor Rotor Geometry Input . . . . . . . . . . . . . . . . . . . . . . . . 80

Appendix B MANUAL: FAST MULTIROTOR PERFORMANCE PREDICTION PRO-

$\begin{array}{ll}\text { GRAM } & 81\end{array}$

B.1 FMPP Startup . . . . . . . . . . . . . . . . . . . . . . . . 82

B.1.1 File and Folder Startup . . . . . . . . . . . . . . . . . . . . . 82

B.1.2 Input File Selection $\ldots \ldots \ldots \ldots \ldots \ldots \ldots$

B.1.3 Input File Setup . . . . . . . . . . . . . . . . . . . . . . 84

B.1.4 Rotor Folder Setup . . . . . . . . . . . . . . . . . . . . . . . 89

B.1.5 $\quad$ Setting Up Lookup Tables Using BEMT Rotor Analysis Code. . . . . . . . . . . . 92

B.1.6 Analysis Flags $\ldots \ldots \ldots \ldots \ldots$

B.1.7 Adding a Mass Offset $\ldots \ldots \ldots \ldots$. . . . . . . . . . . . . 96

B.2 Running the FMPP Program $\ldots \ldots \ldots \ldots \ldots \ldots$

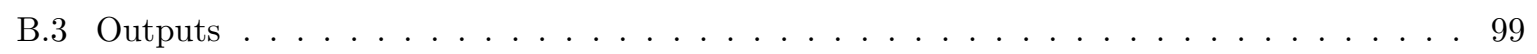

B.3.1 Rotor Forces and Moments . . . . . . . . . . . . . . . . . . . 100

B.3.2 Drag and Body Lift Forces $[\mathrm{N}]] \ldots \ldots \ldots \ldots$. . . . . . . . . . . . . . . . . . .

B.3.3 Moments of Components $[\mathrm{Nm}]$. . . . . . . . . . . . . . . . . . 102

B.3.4 Interference Velocity . . . . . . . . . . . . . . . . . . . . . . . . 103

B.3.5 Power and Pitch Output Variables . . . . . . . . . . . . . . . . . . . . . . . 104

\begin{tabular}{|l|l|}
\hline Appendix C ROTOR PERFORMANCE PREDICTION DATA & 105
\end{tabular}

C.1 3DR Solo Rotor Performance Tables . . . . . . . . . . . . . . . . . . . . . . . . 105

C.2 T-Motor Rotor Performance Tables . . . . . . . . . . . . . . . . . . . . . 115

\begin{tabular}{lr}
\hline References & 128
\end{tabular} 


\section{List of Tables}

3.1 Wake interference model test input variables. . . . . . . . . . . . . . . . . . 27

$4.1 \quad$ Atmospheric conditions during SkyRanger flight testing . . . . . . . . . . . . . . . 44

4.2 Steady state criteria for flight test data $\ldots \ldots \ldots \ldots \ldots \ldots$ 


\section{List of Figures}

$1.1 \quad$ Examples of commercial multirotor unmanned aerial vehicles. . . . . . . . . . . . . . . 2

2.1 Forward flight configuration of quadrotor vehicles. $\ldots \ldots \ldots \ldots \ldots \ldots$

$2.2 \quad$ Hover, roll, pitch and yaw control about longitudinal, lateral, and vertical axes. . . . . . . 8

2.3 Comparison between prediction and experimental thrust coefficient for a T-Motor 18x6.1

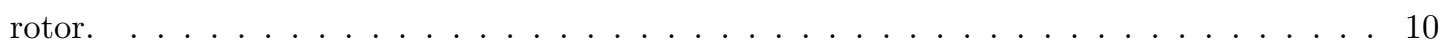

2.4 Comparison between prediction and experimental power coefficient for a T-Motor 18x6.1

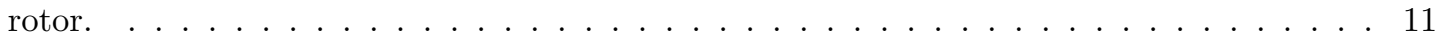

2.5 Coordinate system of the tip path rotor plane and the azimuth station positions relative to the freestream velocity, $V_{\infty}$. . . . . . . . . . . . . . . . . . . . . . . . . . . . . . 12

$2.6 \quad$ Flow field around a hovering rotor generated using wake model $[14$.$] . . . . . . . . 13$

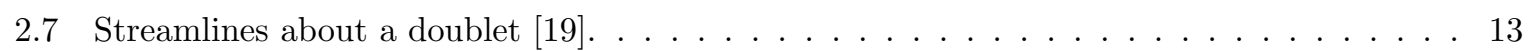

2.8 Fuselage interference velocity components, $q_{t}$ and $q_{n}$, applied to lead and rear rotor hubs in diamond configuration. . . . . . . . . . . . . . . . . . . . . 14

2.9 Example of negative moment applied to multirotor vehicle due to the moment generated by the parasitic drag force of the payload. . . . . . . . . . . . . . . . 15

2.10 Comparison of moment contributions between a non-trimmed and trimmed quadrotor. . . 15

$3.1 \quad$ Free-body diagram of aerodynamic forces on a multirotor vehicle. $\ldots \ldots \ldots \ldots$

3.2 Force trim algorithm. . . . . . . . . . . . . . . . . . . . . . 20

3.3 Standard multirotor components A) rotor, B) motor, C) rotor arm, D) landing gear (leg),

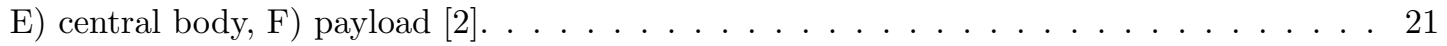


3.4 Resultant velocity vector, $V_{R}$, as a summation of freestream, body interference, and mutual interference velocity vectors. . . . . . . . . . . . . . . . . . . 23

3.5 Example of vortex rings of forward travelling rotor. . . . . . . . . . . . . . . . 24

$3.6 \quad$ Normal components of induced velocity through seven lateral sections of the rotor plane

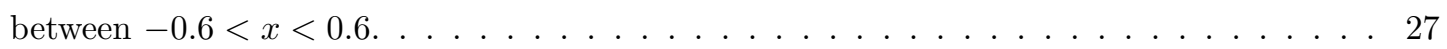

3.7 Normal components of induced velocity through seven lateral sections of the rotor plane between $-0.6<x<0.6 . \ldots \ldots \ldots \ldots \ldots \ldots \ldots \ldots \ldots \ldots \ldots$

3.8 Radial and tangential flow components applied to points $P_{1}$ and $P_{3} . \ldots \ldots \ldots$

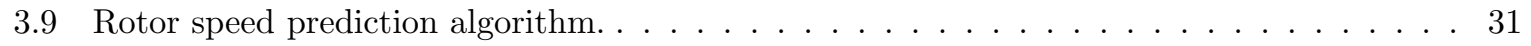

3.10 Implementation of multirotor vehicle performance model. $\ldots \ldots \ldots$. . . . . . . . . 36

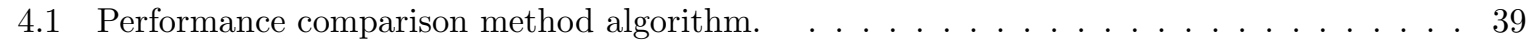

4.2 Reference rotor hub radius and height for 3DR Solo vehicle. . . . . . . . . . . . . . . 40

4.3 Rotor types compared in rotor performance comparison. . . . . . . . . . . . . . 40

4.4 Rotor performance comparison between 3DR Solo 10x4.5 wind tunnel results and GRCSP

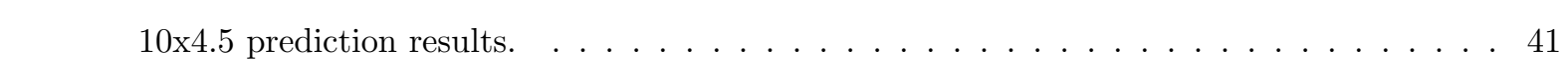

4.5 3DR Solo vehicle experimental and prediction lift and drag results with equal rotor speeds. 42

4.6 Experimental [20 and prediction of pitching moment of 3DR Solo vehicle. . . . . . . . . 43

4.7 Rotor speed comparison between flight test and prediction data of a SkyRanger vehicle. . 45

4.8 Vehicle power comparison between flight test and prediction data of a SkyRanger vehicle. 46

4.9 Vehicle pitch comparison between flight test and prediction data of a SkyRanger vehicle. . 47

5.1 Rotor thrust, speed, and power required for straight and steady longitudinal flight over a range of flight speeds in diamond and square configurations. . . . . . . . . . . . 50

5.2 Rotor thrust, speed, and power required for straight and steady longitudinal flight over a range of flight speeds in diamond and square configurations. . . . . . . . . . . . . 53

5.3 Total vehicle power in diamond and square configurations. . . . . . . . . . . . 54

5.4 Rotor interference velocities applied normal to the rotor plane of each rotor in diamond

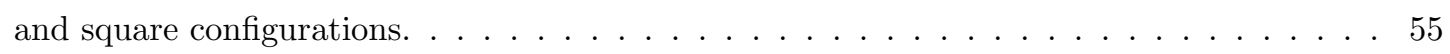

5.5 Induced velocities at each rotor of a vehicle in diamond and square configuration. . . . . 56 
$5.6 \quad$ Fuselage interference velocities at rotor hubs of the diamond and square configurations. Velocities are in rotor reference frame. $\ldots \ldots \ldots \ldots \ldots \ldots$. . . . . . . . . . 59

5.7 Body interference velocity applied to point of interest at distance, $r$, and angle, $\theta$, about a sphere with radius, $R$. Velocities in horizontal reference frame. . . . . . . . . . . . 60

5.8 Inflow velocity components tangent and normal to rotor plane. Vehicle in diamond con-

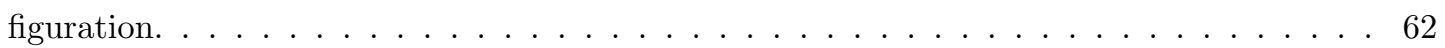

5.9 Inflow velocity components tangent and normal to rotor plane. Vehicle in square config-

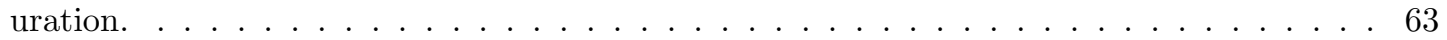

5.10 Change in inflow angle due to flow interference effects compared to vehicle pitch angle. . . 64

5.11 Residual moment components of the SkyRanger vehicle over a range of forward flight speeds. 66

5.12 Difference between diamond and square pitching moment results. . . . . . . . . . . . 67

5.13 Example of negative yawing moment, $M_{z}$, applied to multirotor vehicle due to residual yawing moments generated by the inplane rotor forces, $F_{y}$. . . . . . . . . . . . . . . 69

6.1 Quadrotor in diamond flight with mass, $\mathrm{m}$, attached to lead rotor support arm. . . . . . . 71

6.2 Rotor speed comparison between prediction and flight test results of a SkyRanger vehicle with a mass offset. . . . . . . . . . . . . . . . . . . . . . . . . . . . . . . . . 72

6.3 Comparison between flight test and prediction total vehicle power results for SkyRanger with a mass offset. . . . . . . . . . . . . . . . . . . . . . . . . . . . . 73

6.4 Comparison between flight test and prediction pitch results for a SkyRanger with a mass

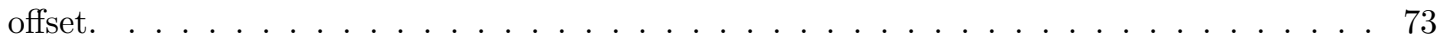




\section{List of Symbols}

\begin{tabular}{|c|c|}
\hline$A$ & rotor disc area, $\mathrm{m}^{2}$ \\
\hline$C_{P}$ & power coefficient \\
\hline$C_{T}$ & thrust coefficient \\
\hline$C_{F x}$ & normal force coefficient \\
\hline$C_{F y}$ & side force coefficient \\
\hline$C_{M x}$ & roll moment coefficient \\
\hline$C_{M y}$ & pitch moment coefficient \\
\hline$C_{Q}$ & torque coefficient \\
\hline$d_{\text {rotor }}$ & rotor diameter, $\mathrm{m}$ \\
\hline$D_{\text {par }}$ & parasitic drag, $\mathrm{N}$ \\
\hline$D_{\text {ind }}$ & induced drag, $\mathrm{N}$ \\
\hline$\vec{F}_{i}$ & force component, $\mathrm{N}$ \\
\hline$F_{x}, F_{y}, F_{z}$ & Total force vector components, $\mathrm{N}$ \\
\hline$l$ & length, m \\
\hline$L$ & lift, $\mathrm{N}$ \\
\hline$m$ & mass, $\mathrm{kg}$ \\
\hline$q$ & dynamic pressure, $\mathrm{N} / \mathrm{m}^{2}$ \\
\hline$r$ & radius, $\mathrm{m}$ \\
\hline$\vec{r}_{i}$ & moment arm component, m \\
\hline$\Re_{i}$ & component residual moment, $\mathrm{Nm}$ \\
\hline rpm & rotor speed, rev/min \\
\hline$n$ & rotor speed, rev/sec \\
\hline$T$ & thrust, $\mathrm{N}$ \\
\hline \multicolumn{2}{|c|}{$v_{b o d y, x}, v_{b o d y, y}, v_{b o d y} f_{y}$ uselage interference velocity components, $\mathrm{m} / \mathrm{s}$} \\
\hline$v_{i n t, x}, v_{i n t, y}, v_{i n t, z}$ & rotor interference velocity components, $\mathrm{m} / \mathrm{s}$ \\
\hline$v_{i}$ & rotor self-induced velocity, m/s \\
\hline$V_{\infty}$ & freestream velocity, $\mathrm{m} / \mathrm{s}$ \\
\hline$V_{R}$ & resultant velocity, $\mathrm{m} / \mathrm{s}$ \\
\hline
\end{tabular}




$\begin{array}{ll}W & \text { weight, } \mathrm{N} \\ x_{B}, y_{B}, z_{B} & \text { body coordinates } \\ x_{S}, y_{S}, z_{S} & \text { flight path coordinates } \\ x_{H}, y_{H}, z_{H} & \text { local-horizontal coordinates } \\ \alpha & \text { angle of attack, deg } \\ \alpha_{i n t} & \text { change in angle of attack due to interference velocity, deg } \\ \alpha_{R} & \text { resultant angle of attack, deg } \\ \gamma & \text { flight path angle } \\ \lambda & \text { rotor inflow ratio } \\ \mu_{\infty} & \text { advance ratio } \\ \theta, \phi, \psi & \text { pitch, roll and yaw angles, deg } \\ \rho & \text { air density at altitude, } \mathrm{kg} / \mathrm{m}^{3} \\ \Psi & \text { azimuth angle, deg } \\ & \text { rotor angular velocity, rad } / \mathrm{s} \\ & \end{array}$




\section{CHAPTER 1}

\section{INTRODUCTION}

\subsection{Small Multirotor Aerial Systems}

Small multirotor unmanned aerial vehicles are vertical take off and landing (VTOL) aircraft that use multiple rotors and typically have four-, six- or eight- rotors. Four-, six- and eight- rotor vehicles are also respectively referred to as quad-, hexi-, and octo- rotors or -copters in industry. Figure 1.1 shows examples of quadrotor and octocopter style multirotor vehicles. Rotors about a central body spin in clockwise and counter-clockwise directions at varying rotational speeds in order to control the flight speed, altitude, and attitude of the vehicle. The central body houses the flight guidance, navigation, control, and power systems of the vehicle and provides a mounting surface for an attachable payload. Payloads are for example, cameras or package handling devices depending on customer needs of the multirotor vehicle.

Example applications of camera payloads are photography, surveillance for law enforcement and the military, crop yield analysis in agriculture, structural analysis and surveying for civil engineering, as well as aerial photography used for humanitarian relief efforts [1]. Logistics and delivery companies are currently developing drone door-to-door delivery services that include delivering customer packages using multirotor vehicles. Delivery multirotor vehicles require the vehicle design to be able to operate with package payloads of different weights and sizes. Multirotor designers may also want to offer a customers the option to choose between different camera or package handling device types that can attach to the same base vehicle. Adding interchangeable payload capabilities to a multirotor vehicle creates 
performance variability due to changing vehicle weight, centre of gravity location, and aerodynamic drag due to the payload shape and size. Changes in drag and weight ultimately change thrust and pitch requirements in order to maintain trimmed flight.

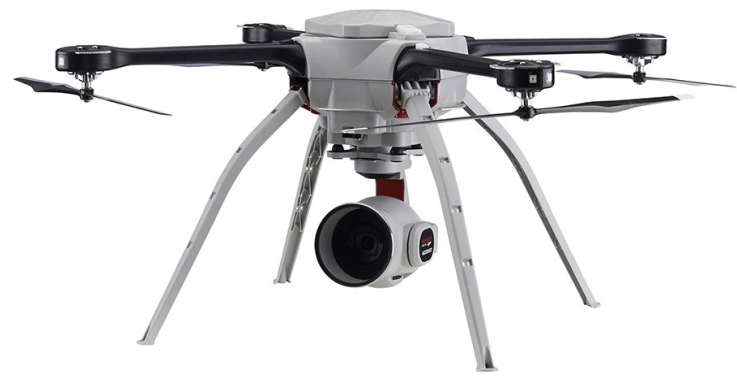

(a) Aeryon SkyRanger quadcopter [2].

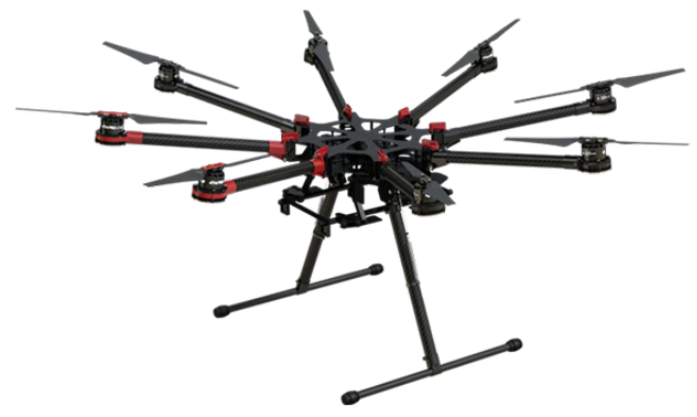

(b) DJI Spreading Wing S1000 octocopter [3].

Figure 1.1: Examples of commercial multirotor unmanned aerial vehicles.

For multirotor vehicles, only rotational speeds of each rotor can be modified in order to trim the vehicle. Rotor speeds are controlled by the control system of the vehicle. Thrust for control, however, also depends on the inflow conditions. Inflow conditions are the inflow velocity applied to the rotor at a specific inflow angle. Inflow conditions are based on the freestream velocity, crosswind components, interactions due to the aerodynamics of the other rotors, and due to the fuselage and freestream conditions. Using aerodynamic models that predict the changing inflow conditions improves the reliability of the multirotor control systems.

The development of the control systems for multirotor vehicles traditionally rely on relatively simplistic aerodynamic models. Examples of the simplistic aerodynamic models include simple quadratic relationships between thrust and rotor-rotational speeds that neglect effects such as advance ratio, inflow conditions, and the impact of the other rotors and fuselage whose presence alter the local flow field 44. All of these results lead to highly nonlinear aerodynamic responses of multirotor vehicles that are difficult to fully capture using the simplistic approaches. Hoffmann et al. noted in their quadrotor flight testing that the precision of trajectory control was directly dependent on the quality of the aerodynamic models [5. This was found to be particularly true with increasing flight speed.

Rather than using more comprehensive aerodynamic models, the control laws are often developed 
using extensive flight testing, which is relatively resource intensive. Powers et al. conducted flight testing of a kQuadNano quadrotor in order to determine the errors in vehicle orientation, forward velocity, and angular velocity when comparing thrust of a baseline propeller tests in still air and thrust for a vehicle in forward flight [4]. The authors identified that the main control model of a multirotor vehicle is a complex function of motor speeds and environmental conditions. The errors determined through flight testing became constants in the thrust prediction models. Incorporating comprehensive aerodynamic models in the control laws of autonomous multirotor vehicles can reduce the flight testing time that is required to determine the model errors. In addition, a comprehensive aerodynamic model can also assist in the rotor speed prediction for multirotors in any configuration prior to initial flight testing.

Recent multirotor vehicle performance research uses more sophisticated aerodynamic models for the rotors, for example Refs. 6, 7, 8, 9, 10. These references use a first principles approach and implement rotor performanace models including blade-element momentum theory, vortex lattice, and computational fluid dynamics (CFD) methods to predict the performance of multirotor vehicles rather than relying on flight testing. For example, Ref. 7 explored using blade-element momentum theory and vortex lattice method to predict forces and moments for small propellers exposed to nonaxial flow conditions. The paper compared rotor force and moment predictions with experimental data generated from wind tunnel testing of small propellers. The axial force predictions captured the experimental axial force results well and slightly overpredicted rotor side forces and rolling moments. By further developing these more complex aerodynamic models, such as the models used in Ref. 7, performance traits of different small rotor designs can be assessed prior to manufacturing or testing efforts. Nevertheless, most multirotor performance prediction methods still lack the ability to fully capture the complex aerodynamics that result from the rotor wake interactions of several rotors that operate in close proximity and the impact of different payload types.

The study of rotor wakes of rotary blades goes back to the early 1950s. Early rotor wake research explored analytically helicopter rotor wakes and wake interactions of coaxial and tandem rotors. In 1954, Dingeldein showed that helicopter rotors in coaxial and tandem rotors have greater power requirements compared to single rotor power measurement of the same thrust coefficients [1]. Particularly, the rear rotor of a tandem configured helicopter in level flight required $50 \%$ or more power than the front rotor in order to maintain the same thrust coefficient. In contrast, the front rotor showed power requirements similar to the single rotor. Castles and De Leeuw in 1954 and 1956 developed analytical methods to 
calculate the normal component of induced velocities at the rotor plane [12. Their method used a Biot-Savart approach using an integral solution of wakes in the form of a uniform cylinder. Castles and De Leeuw also noted that the interference velocity applied to the rear rotor of a tandem-rotor helicopter should be considered when calculating the longitudinal stability characteristics of a vehicle. By the end of the 1950s, Heyson provided a summary of the research relating to induced flow of helicopter rotors 13. Methods compared spiral, vortex-ring, and cylindrical wake methods and the assumptions that are made for these methods. Using rotor wake prediction methods aim to advance the performance prediction capabilities of aerodynamic models used in the controls systems of todays multirotor vehicles.

Recent studies of rotor wake interactions of multirotor vehilce performance have been explored. The multirotor rotor vehicle performance model from Ref. 14 used a wake interference model that is based on the method adapted from Castles and DeLeeuw in Refs. 12 and 15 to estimate the cumulative wake interference on each rotor. The rotor wake interaction model predicted the changes in inflow conditions of the rotors in a multirotor configuration by using a ring wake model to solve for the induced velocities from one rotor on the surrounding rotors. The model used a discretized method of the cylindrical wake approach aimed to be computationally fast when solving for the induced velocities. Another attempt to look into the mutual interference of rotor wakes on a multirotor vehicle was done by Luo et al. in Ref. 9 using a circular fixed-wing analogy to predict rotor induced velocities. This approach, however, showed to have limitations when comparing subsequent thrust coefficient predictions to computational fluid dynamics predictions. The fixed-wing analogy stems from the equation for the lift of a circular wing being the same as the Glauert's high speed approximation method to predict thrust of a rotor [16. Diaz and Yoon studied the aerodynamics of two commercially available quadrotors using highfidelity CFD models to fully account for the aerodynamic interactions of several rotors [10]. The authors reasoned that using high-fidelity CFD models is preferable over using low-fidelity models, such as Euler solvers, when investing flow interactions and their impact on multirotor vehicle performance. However, even using the processing power of two of NASA's supercomputers, Pleidas and Electra, required two days to converge quasi-steady solutions for selected airspeed, vehicle pitch and rotor speed. Although CFD is a useful tool to determine accurately the effects of rotor wake and body interactions on the overall vehicle performance, the designer of a multirotor vehicles requires a fast performance prediction method in order to quickly determine the changes in vehicle performance characteristics due to vehicle configurations changes. 
In this thesis, a fast multirotor vehicle flight performance prediction method is presented that provides rotor speed trim solutions over a range of airspeeds in steady level flight. The steady state solutions include vehicle pitch, rotor thrust, rotor speed, rotor power, and inflow velocities and inflow angles influenced by mutual rotor interference and central body interference. The multirotor vehicle performance prediction is an expansion of the model presented in Ref.14. The method determines rotor speeds required to trim the aircraft for forces and moments in the longitudinal plane. Overall, the method is computationally fast and gives designers insight about the aerodynamics and stability characteristics of various multirotor vehicle designs and payload configurations.

This thesis describes the aerodynamic and the flight dynamics principles of multirotor vehicles that control the movement and trim conditions of the vehicle. The program algorithms that these principles are to predict vehicle trim are discussed. Performance comparisons are made between the prediction method, wind tunnel data of a 3DR Solo vehicle presented by NASA AMES, and flight test data from the Aeryon SkyRanger. To show the impact of rotor and fuselage interference velocities on flight performance and the trim solutions of quadrotor vehicles, an orientation study is conducted comparing the flight characteristics between vehicles flying in diamond and square configurations. Finally, a flight test study was conducted where a mass was attached to the lead arm of the SkyRanger vehicle, where the prediction capabilities of the fast multirotor performance prediction method are tested for a vehicle with an asymmetric weight distribution. 


\section{CHAPTER 2}

\section{MULTIROTOR AERODYNAMICS}

\section{AND FLIGHT DYNAMICS}

The performance of multirotor vehicles is influenced by the aerodynamics of their rotors and fuselage. Understanding their aerodynamics is essential for predicting the rotor speeds and power required for the multirotor vehicle to fly in any configuration. The method used in this thesis to predict multirotor vehicle performances consists of a series of models that predict the vehicle and rotor forces and moments, and rotor speed requirements that are needed to maintain steady level flight. The models include a force trim, fuselage interference, rotor speed, moment trim, and power prediction models.

This chapter discusses the aerodynamic principles behind rotor performance, rotor interference, fuselage interference, power, and vehicle trim used in the multirotor vehicle performance prediction method. This chapter first defines the flight configurations and the flight dynamics of quadrotor vehicles, the vehicle type analysed in this thesis. 


\subsection{Configurations}

The most common configuration of a multirotor vehicle has four rotors, also referred to as a quadrotors. Figure 2.1 shows the two types of orientations and rotor numbering on a quadrotor relative to the freestream velocity. Quadrotors have two main configurations, square and diamond, and anything in between. These configurations can also be referred to as "X" and "+" configurations and refer to the number of leading rotors of a quadrotor [1. Square, or "X" configuration, has two leading rotors and diamond, or "+" configuration, has one leading rotor.

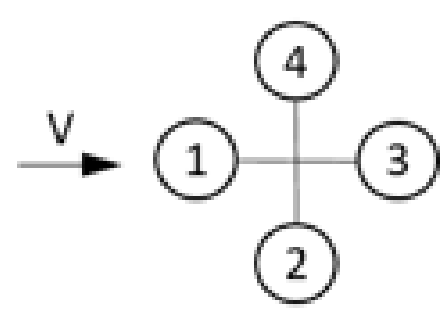

(a) Diamond, '+', configuration.

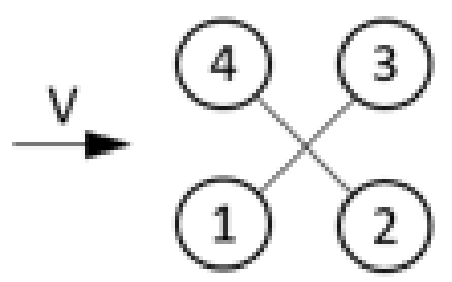

(b) Square, 'x', configuration.

Figure 2.1: Forward flight configuration of quadrotor vehicles.

The operator's preference to fly in square or diamond configurations is generally dependent on if the on board camera is recording or not. When filming, it is undesirable for the rotor blades to be present in the video footage. Therefore, cameras are placed in a position where the video is captured between two rotors and the rotors are outside the video frame. When the cameras are off, it can be advantageous to flying in a diamond configuration.

\subsection{Roll, Pitch, and Yaw Control}

The vehicle speeds and yaw angle are controlled by the onboard control system of a multirotor vehicle. Roll and pitch angles are applied by varying the rotor speeds to change the force and torque balances of the vehicle. Figure 2.2 shows the change in roll, $\phi$, pitch, $\theta$, and yaw, $\psi$, angles about the three axes, $(x, y, z)$, of a standard quadrotor vehicle by varying the thrust, $T$, and torque, $Q$, of each of the fours rotors. In general, the vehicle is symmetrical along the longitudinal plane and the centre of gravity is located within the body somewhere along the vertical axis of the vehicle. 
Figure 2.2a shows four rotors of equal rotor thrusts and torque of equal magnitude but opposite direction for opposing rotors. To increase pitch in forward flight for a quadrotor travelling with two leading rotors, the two trailing rotors increase rotor speed equally resulting in a rotation about the lateral axis, as shown in Fig. 2.2c. Positive pitch is the angle between the freestream velocity vector and the rotor disk plane if the freestream vector is above the rotor disk plane. The pitch is negative if the freestream vector is below the rotor disk plane. Increasing or decrease the rotational speed of the two side rotors in forward flight will result in a roll about the longitudinal axis, as shown in Fig. $2.2 \mathrm{~b}$ Changing the rotor speed of diagonally opposed rotors will rotate the vehicle about the vertical axis, as shown in Fig. 2.2d, In addition, if all rotors increase or decrease their rotor speeds equally, the vehicle will climb and descend, respectively. The onboard control systems adjusts the rotational speeds of the rotors and uses feedback controllers to keep the vehicle travelling along a desired trajectory.

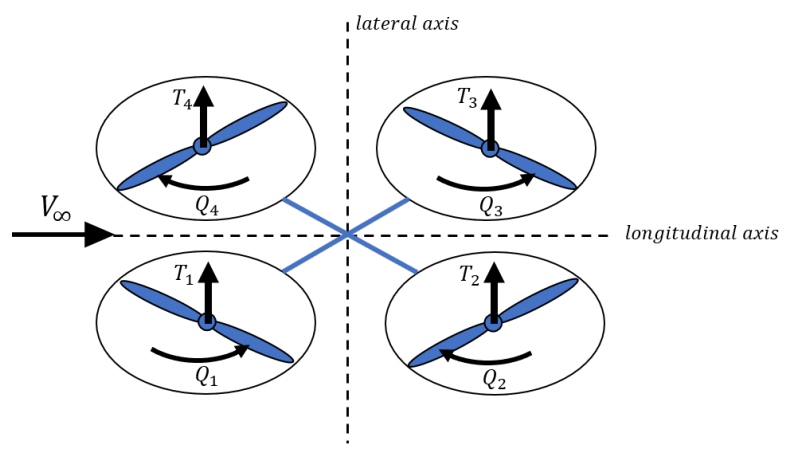

(a) Neutral

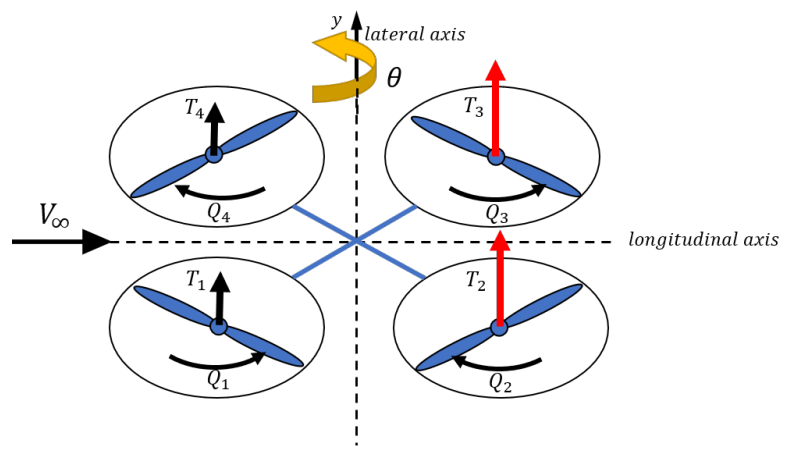

(c) Pitch

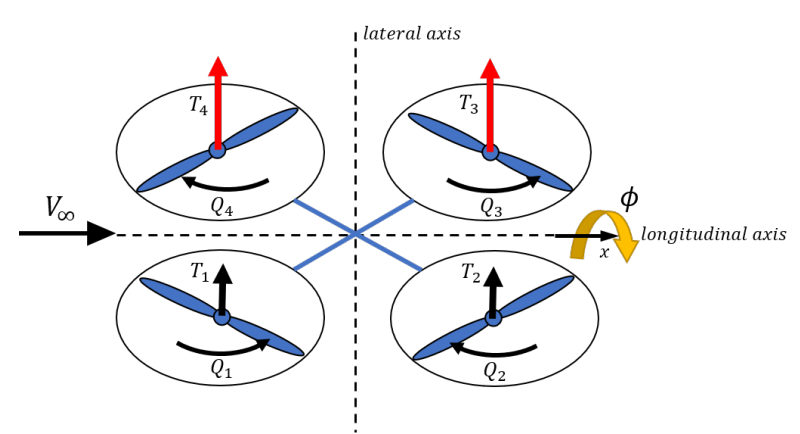

(b) Roll

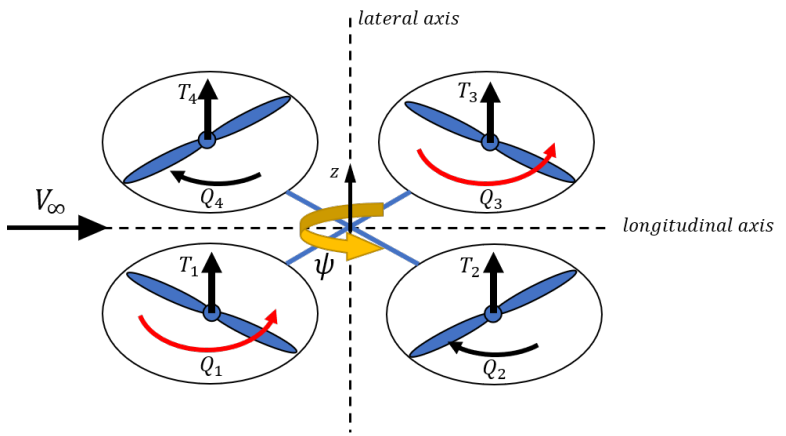

(d) Yaw

Figure 2.2: Hover, roll, pitch and yaw control about longitudinal, lateral, and vertical axes. 


\subsection{Rotor Aerodynamics}

The performance prediction method uses rotor performance lookup tables that were pre-generated using a blade element momentum theory-based model for small fixed pitched rotors [17. The rotor performance prediction combines blade element theory, momentum theory, and linear inflow models to predict rotor forces, moments, and power in hover and forward flight. Rotor thrust, $T$, rotor normal forces, $F_{x}$ and $F_{y}$, rotor moments, $M_{x}$ and $M_{y}$, and rotor torque, $Q$, are calculated using the following rotor convention:

$$
\begin{gathered}
T=F_{z}=C_{T} \rho A(\Omega R)^{2} \\
F_{x}=C_{F_{x}} \rho A(\Omega R)^{2} \\
F_{y}=C_{F_{y}} \rho A(\Omega R)^{2} \\
Q=M_{z}=C_{Q} \rho A \Omega^{2} R^{3} \\
M_{x}=C_{M_{x}} \rho A \Omega^{2} R^{3} \\
M_{y}=C_{M_{y}} \rho A \Omega^{2} R^{3}
\end{gathered}
$$

Rotor power, $P$ is calculated as:

$$
P=C_{P} \rho A(\Omega R)^{3}
$$

where $\rho$ is the density, $A$ is the rotor disk area, $R$ is the rotor radius, and $\Omega$ is the rotor speed in radians per second. Force, moment, and power coefficients are tabulated and organized within the lookup table based on advance ratio, $\mu$, angle of attack, and rotor speed. A linear interpolation is used to interpolate between points in the lookup tables. Advance ratio, $\mu$, is the ratio of freestream velocity, $V_{\infty}$, and tip 
speed, $\Omega R$, calculated as:

$$
\mu=\frac{V_{\infty}}{\Omega R}
$$

Examples of rotor lookup tables are located in Appendix C.

To show the relationship between rotor performance and advance ratio, Figs. 2.3 and 2.4 show experimental and prediction thrust coefficient, $C_{T}$, and power coefficient, $C_{P}$, of a T-Motor $18 \times 6.1$ rotor. The T-Motor 18x6.1 rotor is used as a sample rotor in the orientation study discussed in Chapter 5. Each trend is a function of advance ratio, angle of attack, and rotor speed. The rotor performance at four positive angles of attack and two rotor speeds are compared. The $C_{T}$ results show overlapping experimental and prediction results at $+90^{\circ}$ at advance ratios over $\mu=0.05$ and similar trends for lower angles of attack for a T-Motor $18 \times 6.1$ rotor [17. A rotor at $0^{\circ}$ angle of attack experiences a freestream that is fully parallel to the rotor plane, otherwise referred to as fully edgewise flight. A rotor with a positive angle of attack travels with a freestream flow angled above the rotor plane. Rotors with a significant amount of edgewise freestream component experience increasingly positive forces and moments with increasing advance ratio. The increase in rotor forces and moments is attributed to advancing and retreating blade effects.

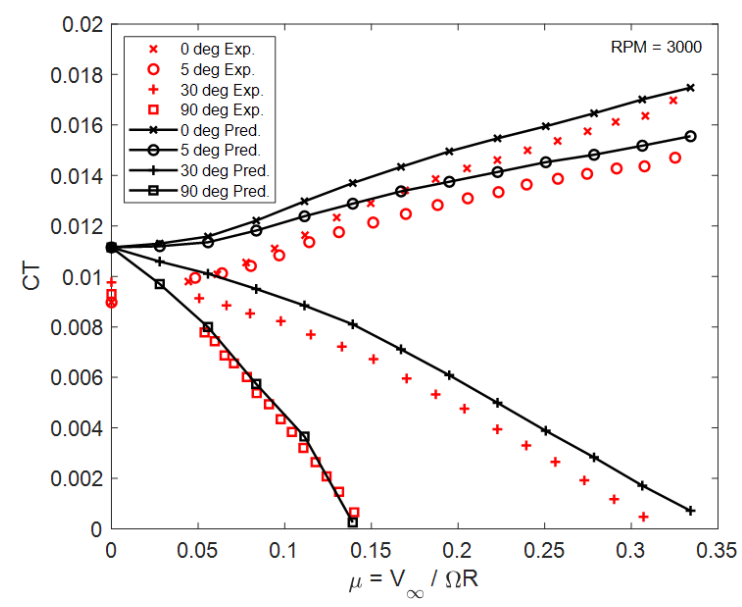

(a) $C_{T}$ at $\mathrm{RPM}=3000$.

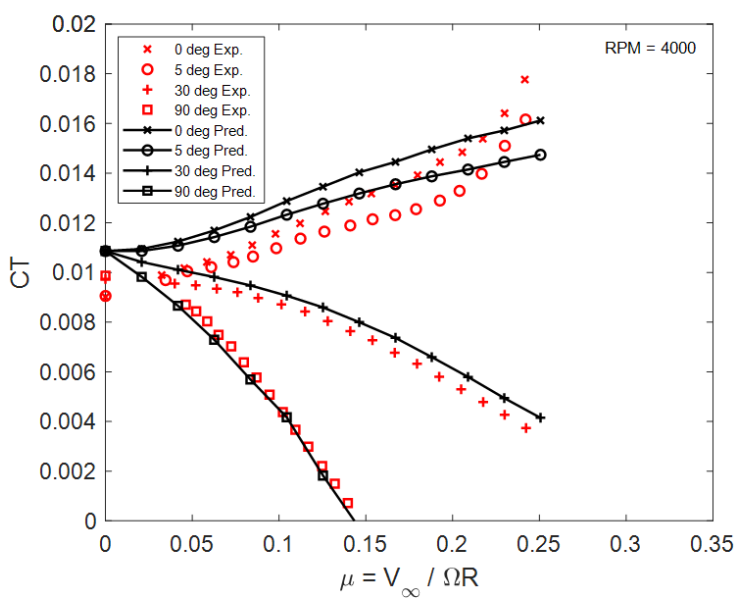

(b) $C_{T}$ at $\mathrm{RPM}=4000$.

Figure 2.3: Comparison between prediction and experimental thrust coefficient for a T-Motor 18x6.1 rotor. 


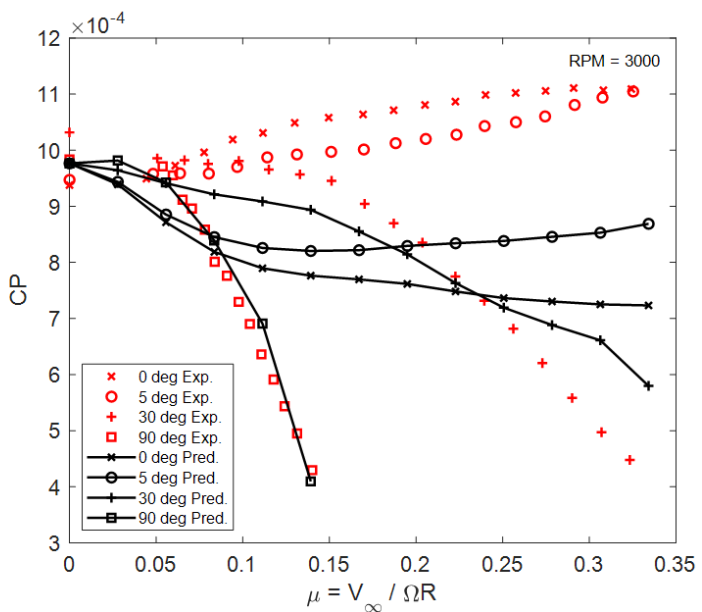

(a) $C_{P}$ at $\mathrm{RPM}=3000$.

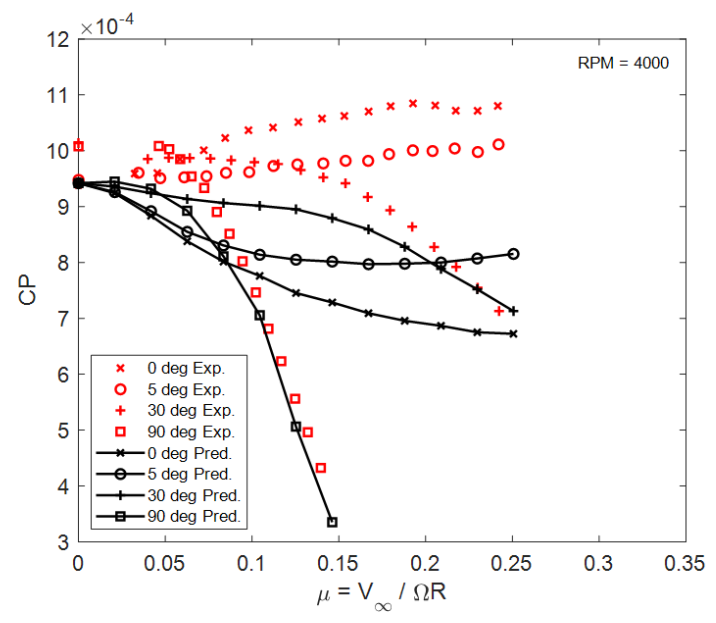

(b) $C_{P}$ at $\mathrm{RPM}=4000$.

Figure 2.4: Comparison between prediction and experimental power coefficient for a T-Motor 18x6.1 rotor.

The performance prediction model uses the plane loads and moments to determine vehicle forces and moments used in the force and moment trim models. Rotating the differential lift and drag components into the rotor plane provides the differential thrust, $d T$, and radial force, $d F$, of the rotor. The radial and axial flow components of rotor sections of four different azimuth stations, $0^{\circ}, 90^{\circ}, 180^{\circ}$, and $270^{\circ}$, of a rotor during fully edgewise flight, $\alpha=0$, are shown in Fig. 2.5. The leading and trailing edges of the rotor disk are at azimuth stations $180^{\circ}$ and $0^{\circ}$, respectively. At $90^{\circ}$, the blade advances into the flight direction whereas $270^{\circ}$ it retreats. At the advancing and retreating locations, the freestream velocity, $V_{\infty}$, vector is added or subtracted to the radial velocity within the rotor plane. Adding the freestream velocity to the radial velocity changes the resultant inflow velocity and resultant inflow angle, ultimately changing the effective angle of attack, $\alpha_{e f f}$, and rotor pitch angle, $\beta$. Adding the radial velocity, $\omega R$, and axial velocity, $V_{A}$, components results in the resultant velocity vector, $V_{R}$, at resultant inflow angle relative to the rotor plane, $\phi$. The axial velocity component is the sum of the normal component of the freestream velocity, $V_{\infty} \sin \alpha$, and the induced velocity through the rotor, $v_{i n d}$. In forward flight, the trailing edge of the rotor at $0^{\circ}$ plane sees a downwash that is stronger than at the leading edge due to a skewed wake. This reduction in axial flow at the edge leads to a greater effective angle of attack of the blade and higher sectional lift, drag, and moment. Rotors with an increasing edgewise freestream component have varying rotor forces and moments. Understanding these changes in rotor 
force and moments with increasing flight speeds becomes essential when predicting the trim solutions of the multirotor vehicle.

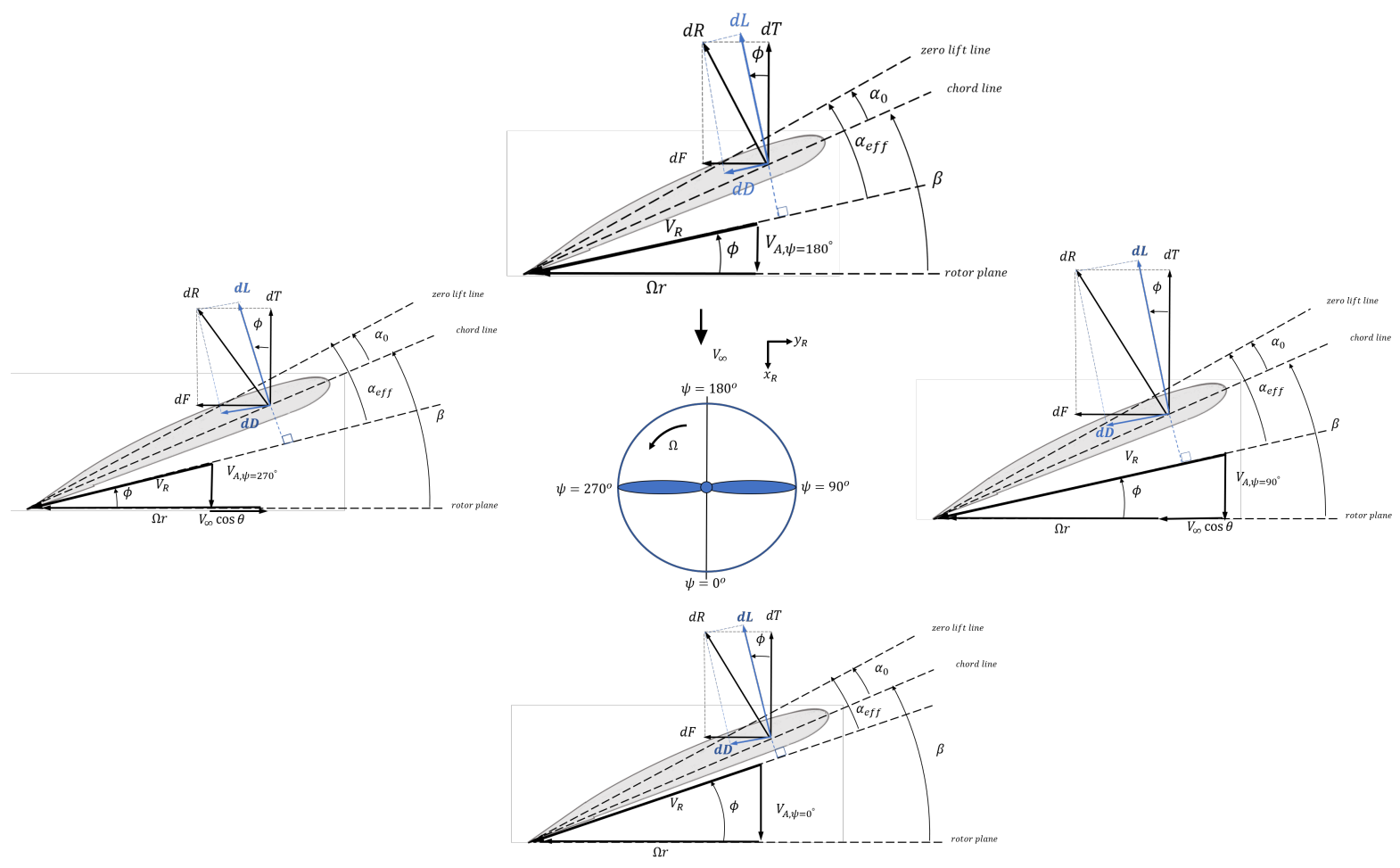

Figure 2.5: Coordinate system of the tip path rotor plane and the azimuth station positions relative to the freestream velocity, $V_{\infty}$.

\subsection{Interference Velocity}

There are two types of interference velocities considered within the multirotor performance prediction method: rotor interference and fuselage interference. The rotor interference velocity is the velocity applied to a rotor as a result of the influence of the flowfields of the other rotors on a multirotor vehicle. Fig. 2.6 shows an example of the flowfield about a hovering single rotor [14]. For a rotor in hover, the flow field around the rotor has the induced velocities flow downward through the rotor plane upward beyond the rotor plane [12, 18. The flowfield about one rotor influences the flowfield of the other rotors in a multirotor configuration. The wake interference model presented in the model presented in Ref. 14 is used to predict the rotor interference. 


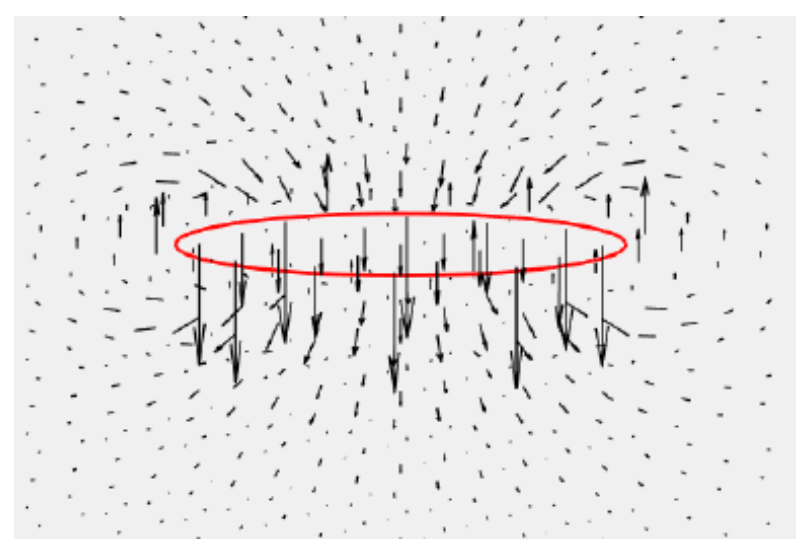

Figure 2.6: Flow field around a hovering rotor generated using wake model [14].

The fuselage interference velocity is the velocity applied to each rotor due to the presence of the central body of the vehicle. Figure 2.7 shows the streamlines about a cylinder, which is similar to the flow about the cross section of a sphere. In the figure, the freestream velocity, $U_{\infty}$, is represented by the arrows showing streamlines of uniform flow. The flow travels about the cylinder and the point at the end of the vector, $r$, will experience an interference velocity due to the changes in the flow about the cylinder. The flowfield about a sphere can be modelled using a point doublet in a uniform flowfield.

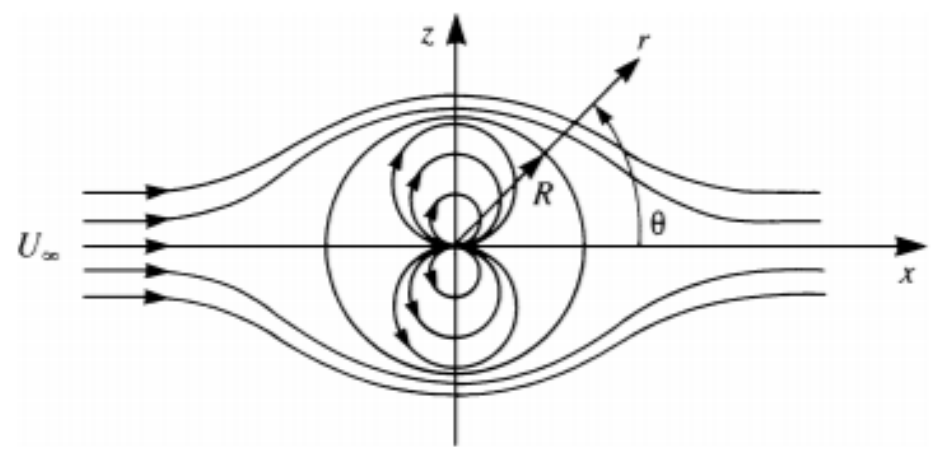

Figure 2.7: Streamlines about a doublet [19.

Fig. 2.8 shows an example of the lead and rear rotors, $P_{1}$ and $P_{3}$ respectively, experiencing normal fuselage interference, $q_{n}$, and tangential fuselage interference, $q_{t}$, velocities. The orientation study in Chapter 5 shows the fuselage interference results in normal and tangential components. 


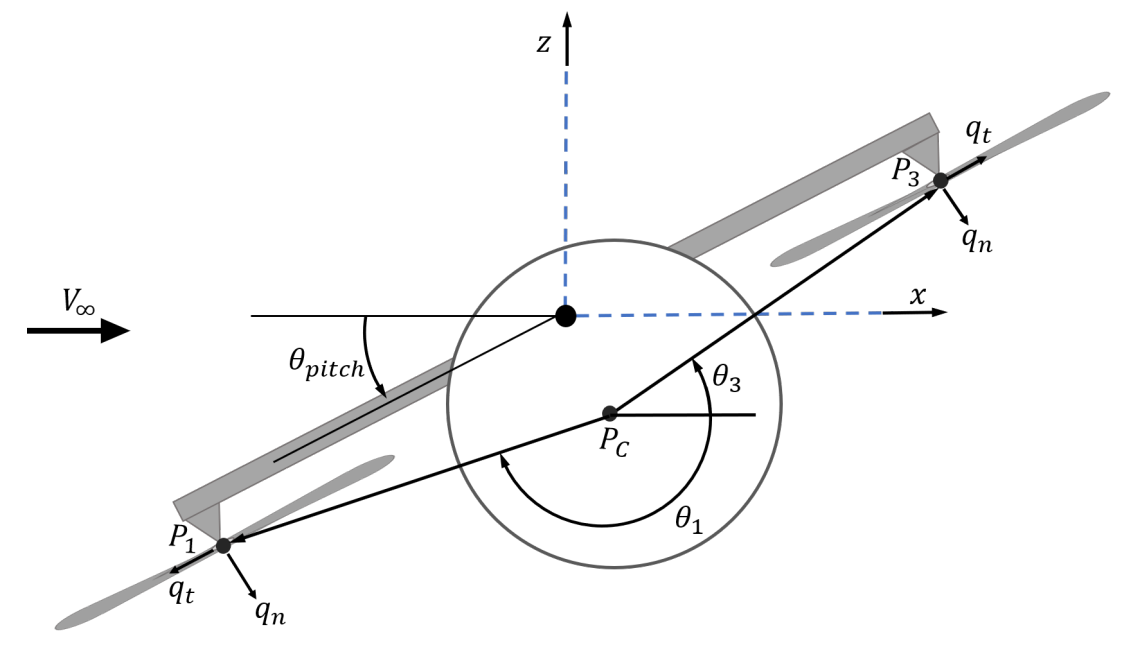

Figure 2.8: Fuselage interference velocity components, $q_{t}$ and $q_{n}$, applied to lead and rear rotor hubs in diamond configuration.

The multirotor vehicle performance prediction method combines the interference velocity vectors to the freestream velocity and updates the inflow conditions of each rotor. The updates in inflow conditions changes the performance of each rotor. The change in rotor performance due to the rotor and fuselage interference is studied in the orientation study in Chapters 5 .

\subsection{Stability, Control, and Trim}

The performance prediction method predicts the moments of produced by the rotors, parasitic drag of the vehicle components, induced drag and lift of the fuselage, and weight components of a multirotor vehicle. For example, Fig. 2.9 shows a multirotor vehicle that experiences a negative moment due to the parasitic drag of the payload. A negative pitching residual moment indicates the component moment causes the vehicle to pitch down. Adding all of the moments together provides non-zero total vehicle moments about the vehicle's longitudinal, lateral, and vertical axes. In order for the vehicle to be in a steady and trimmed flight condition, the total external forces and moments that act on the vehicle must equal zero. To maintain force trim, the total vehicle thrust must counteract the drag, body lift, and weight of the vehicle. To maintain moment trim, the distribution of rotor thrust must counteract the total moments generated by the force and rotor moments to resolve the total vehicle moments to zero. 


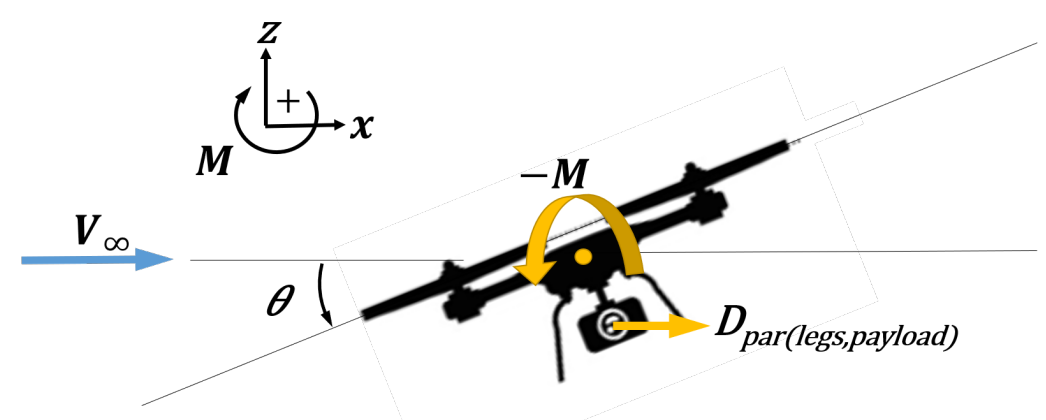

Figure 2.9: Example of negative moment applied to multirotor vehicle due to the moment generated by the parasitic drag force of the payload.

Figure 2.10 shows two examples of the predicted pitching moments of a quadrotor vehicle in nontrimmed and trimmed states. In the non-trimmed state in Fig. 2.10a, the total pitching moment changes between positive to negative moments around $17 \mathrm{~m} / \mathrm{s}$ as the moment due to drag, primarily due to drag of the landing gear, increases more negatively with increasing speed. For the vehicle in a trimmed state, shown in Fig. 2.10b, the thrust distribution between the rotors is set so that the subsequent total vehicle pitching moment is zero and force trim is maintained. The moments due to vehicle differential thrust are represented by the rotor forces trend in the figure.

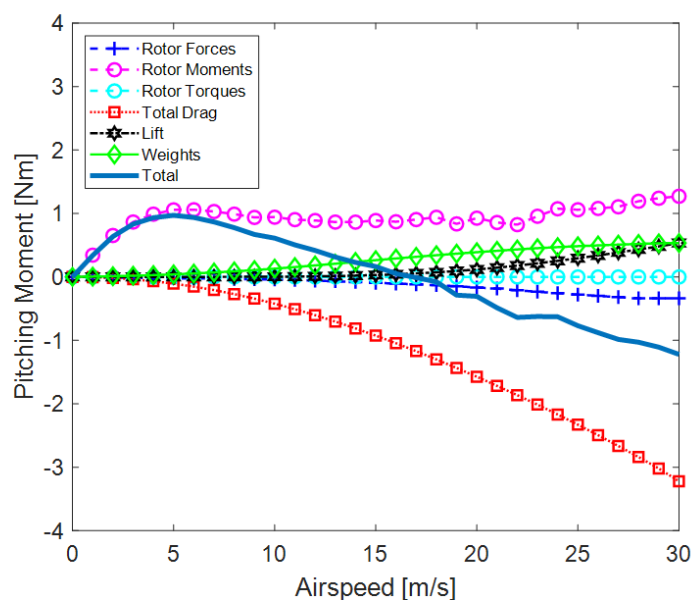

(a) Not trimmed

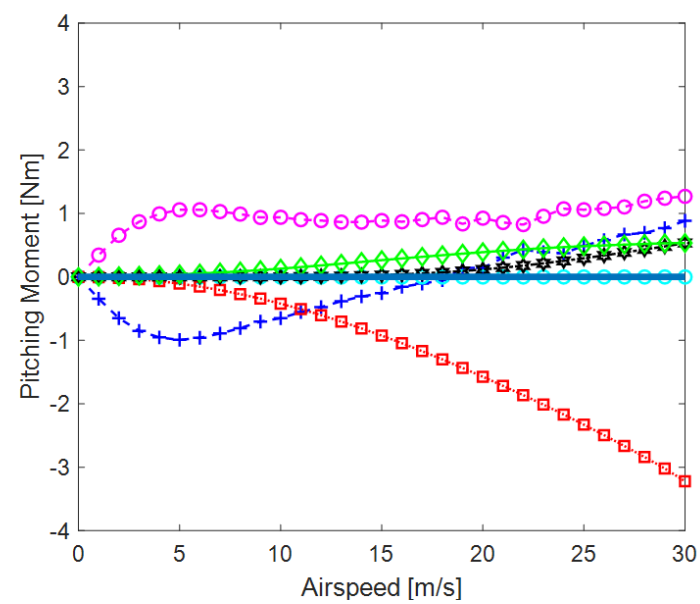

(b) Trimmed

Figure 2.10: Comparison of moment contributions between a non-trimmed and trimmed quadrotor. 
With the ability to predict the multirotor vehicle moments and pitch attitude using the multirotor vehicle performance method, static stability conditions can also be investigated. Multirotor vehicles are inherently unstable. Pitching moment versus pitch angle results from Ref. [20] showed that with increasing pitch, the pitching moment of the vehicle also increases. A multirotor vehicle cannot restore its moment due to a perturbation in angle of attack. This issue of instability effects the control laws of the vehicle to ensure safe and steady flight of the mutlirotor vehicle. The control laws of multirotor vehicles regulate the speeds of each rotor to achieve a desired flight condition. The research from Ref. [20] proposes methods towards measuring and improving the stability of multirotor vehicles.

Reference [20] introduces an experimental method to determine the trim condition of multirotor vehicles. Wind tunnel testing was conducted at NASA Ames for five commercially available quadcopter, and one octocopter, vehicles and their respective rotors. The paper addresses the need for experimental performance data of multirotor vehicles where most published research is about dynamics and control of the vehicle. Using the published vehicle lift, drag and pitching moment data, experimental data from NASA Ames is compared with prediction data generated by the multirotor vehicle performance method. This performance comparison study is presented in Chapter 4 . 


\section{CHAPTER 3}

\section{AERODYNAMIC}

\section{PERFORMANCE PREDICTION}

In this chapter, the expansions and improvements are discussed that were made to the multirotor vehicle performance prediction method in Ref. 14. Improvements made to the prediction method include the addition of a rotor-interference and a fuselage-interference model that predict changes in inflow conditions and impact the rotor performance. Fuselage forces include the parasitic drag of the motors and payload, the induced drag of the central body, and the lift of the central body. These forces are accounted for in the force trim model. Furthermore, the prediction method considers the force and moment contributions of fuselage parts, such as the rotor support arms and landing gear, to trim the vehicle. 


\subsection{Equations of Motion}

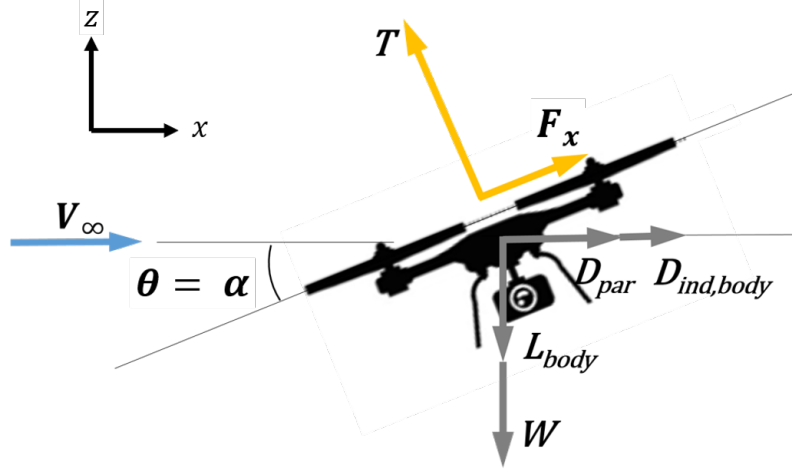

Figure 3.1: Free-body diagram of aerodynamic forces on a multirotor vehicle.

Figure 3.1 shows a free-body diagram of the major forces that act in the longitudinal plane of a multirotor vehicle. During steady and level flight, the loads that the rotor develops, thrust, $T$, and hub drag, $F_{x}$, also referred to as inplane force, must be in equilibrium with the vehicle weight, $W$, and the aerodynamic forces of the fuselage, namely parasitic and induced drag, $D_{p a r}$ and $D_{\text {ind }}$ respectively, and negative lift, $L_{b o d y}$. The equations of motion are based on the set that was developed for the original multirotor vehicle performance method and expanded using the forces of the body lift. Only forces in the longitudinal plane, such as thrust, hub drag, fuselage drag, body lift, and weight are considered for force trim. Other forces, such as the thrust lost due to rotor-support arm interactions, or lift due to support arms or the landing gear are not considered here.

The horizontal and vertical force equilibrium equations of the vehicle are along the freestream direction:

$$
0=D_{\text {par }}+D_{\text {ind,body }}+F_{x} \cos \theta-T \sin \theta
$$

and perpendicular to the freestream direction:

$$
0=T \cos \theta+F_{x} \sin \theta-L_{b o d y}-W
$$


Rearranging Eq. 3.2 for the pitch angle, $\theta$, yields:

$$
\cos \theta=\frac{W+L_{b o d y}-F_{x} \sin \theta}{T}
$$

Substituting Eq. 3.3 into Eq. 3.1 and multiplying all terms by thrust, $T$, yields:

$$
0=T D_{p a r}+T D_{i n d, b o d y}+F_{x}\left(W+L_{b o d y}-F_{x} \sin \theta\right)-T^{2} \sin \theta
$$

Rearranging for $\sin \theta$ gives an expression for pitch angle required for force trim:

$$
\sin \theta=\frac{F_{x} W+F_{x} L_{b o d y}+T D_{p a r}+T D_{i n d, b o d y}}{T^{2}+F_{x}^{2}}
$$

Substituting pitch angle, $\theta$, into Eq. 3.3 yields thrust:

$$
T=\sqrt{\left(W+L_{b o d y}+F_{x} \sin \theta\right)^{2}+\left(D_{\text {par }}+D_{\text {ind,body }}+F_{x} \cos \theta\right)^{2}}
$$

Within the force equilibrium routine, the thrust is assumed to be evenly distributed between the number of rotors.

Figure 3.2 shows the algorithm used to predict the final thrust and pitch requirements of the a force trimmed vehicle. The force trim model uses the total parasitic drag and weight to determine initial thrust and pitch value to use in the performance lookup table. Based on the initial thrust and pitch values, the rotational speed, rotor inplane forces and hub moments are retrieved from the tabulated rotor performance lookup data. Examples of rotor performance tables are located in Appendix C.

With the rotor forces and hub moments known, and the fuselage induced drag and lift calculated using equations given in the next section, the values are used to update thrust and pitch using Eqs. 3.5 and 3.6. The pitch angles of the adjacent iterations are compared and checked for reaching a convergence threshold using the following criteria:

$$
\left|1-\frac{\theta_{i}}{\theta_{i-1}}\right|<=0.01
$$

where $\theta_{i}$ is the latest pitch value and $\theta_{i-1}$ is the previous pitch value. If the pitch comparison is above the threshold, the iterative process continues until convergence is reached. At this point, the final pitch 
value and thrust required for force trim are determined.

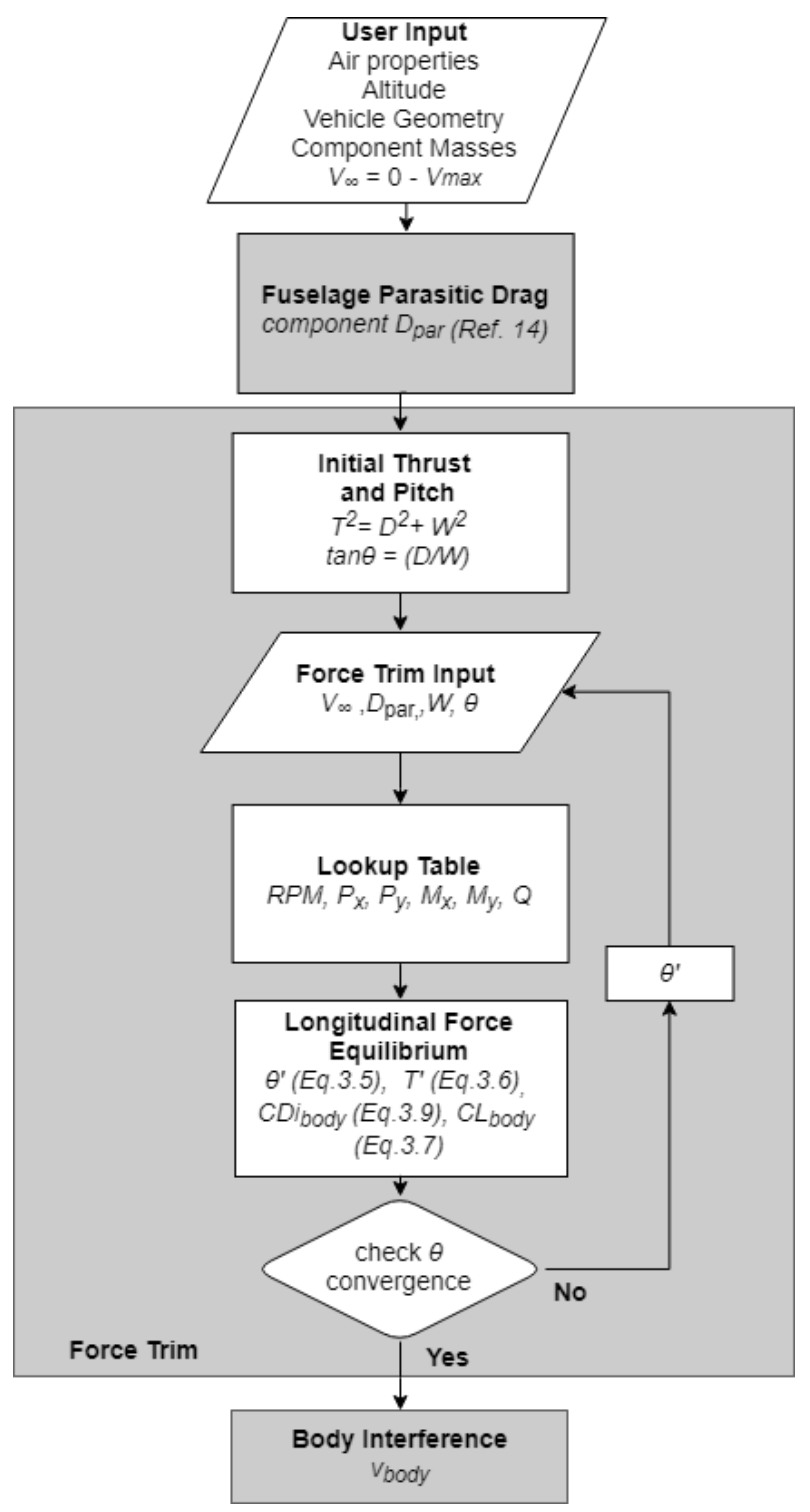

Figure 3.2: Force trim algorithm.

\subsection{Drag and Lift Prediction}

The parasitic drag of the vehicle is predicted using a drag decomposition approach, that is, by adding the drag contributions of each of its elements. The components typical of a multirotor vehicle are shown 
in Figure 3.3 which shows an Aeryon SkyRanger. The parasitic drag model from Ref. 14 is used in the performance prediction model to predict the parasitic drag, $D_{\text {par }}$, of each fuselage component. Assumptions used in the drag prediction model include that the parasitic drag for each component is independent of vehicle pitch and that the drag of each component can be approximated using standard geometries, for example, of cylinders or spheres. For example, the rotor support arms, motors, landing gear and payload are modelled as cylinders. The central body is also approximated as a sphere, although previous wind-tunnel tests indicated that this particular configuration produces a downward force during flight and, subsequently, induced drag [21].

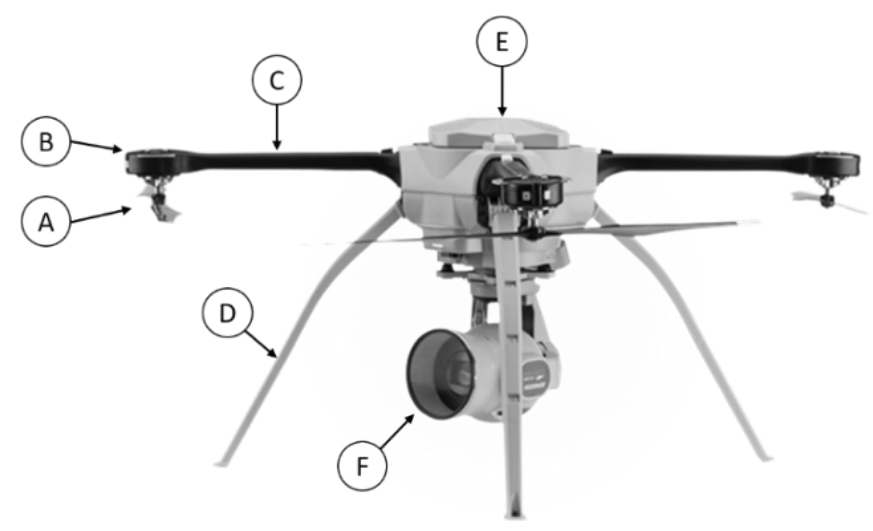

Figure 3.3: Standard multirotor components A) rotor, B) motor, C) rotor arm, D) landing gear (leg), E) central body, F) payload [2].

Based on experimental results in Ref. 14, current analytical predictions for body lift and drag forces are included Eqns. 3.5 and 3.6 . Since the central body does not have a perfectly spherical shape, body lift is approximated using the shape of a circular wing. The coefficient of lift of that wing that has an aspect ratio $A R=4 / \pi$ is:

$$
C_{L}=\frac{C_{l_{\alpha}} A R}{2+\sqrt{A R^{2}+4}} \alpha
$$

where the sectional lift curve slope, $C_{l_{\alpha}}$, is:

$$
C_{l_{\alpha}}=2 \pi
$$


The same tests conducted at Ryerson University indicated that the drag can be estimated relatively accurately using the drag of a sphere plus the lift related drag of a circular wing [14]. The lift related drag of a circular wing is the induced drag of the body defined as:

$$
C_{D_{i}}=\frac{\left(\alpha C_{L_{\alpha}}\right)^{2}}{\pi A R e}
$$

where $\alpha$ is the vehicle pitch attitude and $e$ is the span efficiency factor, which is close to one for circular wings. The total drag coefficient of the vehicle is the sum of the total parasitic drag, $C_{D_{p a r}}$, and the induced drag of the body:

$$
C_{D}=C_{D_{p a r}}+C_{D_{i}}
$$

Under normal flight condition the vehicle has a pitch down orientation, which means that the lift produced by the body is downwards. This extra lift force can be as much as $10 \%$ of the vehicle weight 21].

\subsection{Resultant Inflow Velocity}

The performance of each rotor of a multirotor vehicle greatly depends on the inflow velocity vector, which is impacted by the freestream velocity, the flowfield about the other rotors, and flowfield about the fuselage. The multirotor vehicle performance prediction method uses a rotor interference model to predict the velocities induced on a rotor based on the induced velocities present at each rotor by the other rotors. These induced velocities are called mutual interference velocities because the induced velocities generated by each rotor impact the performance of each other. The prediction method also considers a fuselage interference velocity referred to as fuselage-interference.

To capture the velocity influenced by the fuselage and surrounding rotors in the rotor performance results, the freestream, rotor interference, and fuselage interference velocity vectors are added together to update the inflow velocity and inflow angle used in the lookup table scheme. In the present work, it is assumed that the inflow condition at the rotor hub is representative for the entire rotor disk. This is, obviously, a simplification but was found accurate for ease of implementation. Figure 3.4 shows the freestream, $V_{\infty}$, fuselage interference, $v_{i n t, b o d y}$, rotor interference, $v_{i n t, z_{R}}$, and resultant, $V_{R}$, velocity 
vectors in the rotor, $R$, and horizontal, $H$, reference frames. The positive inflow velocities parallel to the rotor plane indicate flow in the positive $x_{R}$ direction. Negative velocities through the rotor plane indicate a downwash applied to the rotor in the negative $z_{R}$ direction.

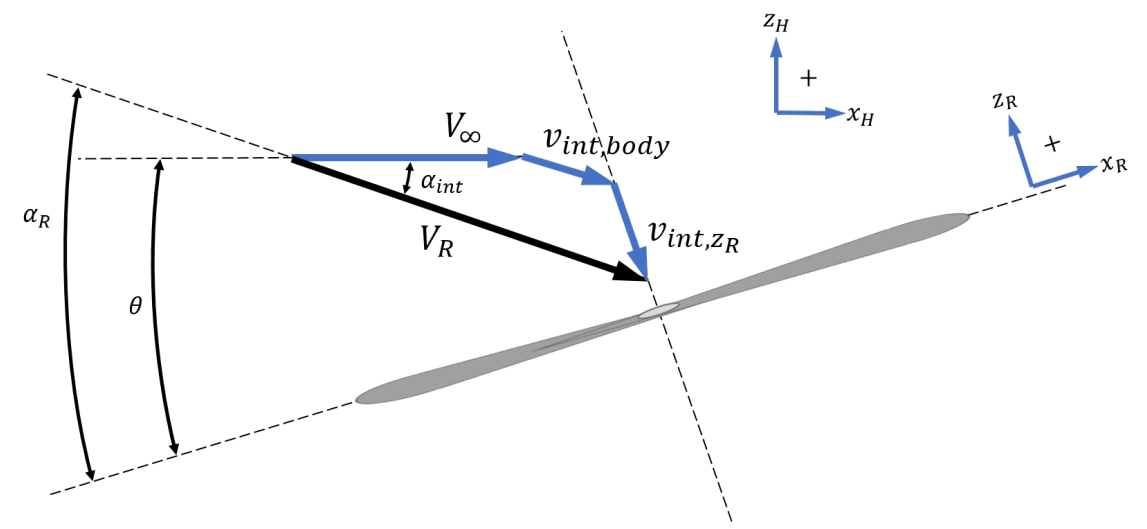

Figure 3.4: Resultant velocity vector, $V_{R}$, as a summation of freestream, body interference, and mutual interference velocity vectors.

The resultant velocity, $V_{R}$, and the resultant angle of attack, $\alpha_{R}$, in the longitudinal plane are calculated using:

$$
\begin{gathered}
V_{R}=\sqrt{\left(V_{\infty}+v_{i n t, b o d y, x}-v_{i n t, z} \sin \theta\right)^{2}+\left(v_{i n t, b o d y, z}+v_{i n t, z} \cos \theta\right)^{2}} \\
\alpha_{R}=\theta-\alpha_{i n t}
\end{gathered}
$$

where $V_{R}$ is the resultant inflow velocity, $\theta$ is the vehicle pitch, and $\alpha_{i n t}$ is the change in angle from the addition of the velocity vectors.

In the following subsections, the rotor interference and fuselage interference models are discussed and how these are used to predict their respective velocity vectors. The section concludes with describing the iterative process using the interference models to predict rotor speeds and rotor powers for a given thrust and new inflow conditions. 


\subsubsection{Rotor Interference}

The rotor interference model predicts the changes in inflow conditions experienced by the rotors of a multirotor configuration by using a ring wake model to solve for the induced velocities from one rotor on the surrounding rotors. The ring wake model was adapted from the analytical method used in Refs. 12 and 15. The model uses a discretized method of the cylindrical wake approach aimed to be computationally fast when finding the induced velocities. Figure 3.5 shows an example of four vortex rings each containing 10 vortex segments below a rotor. For each rotor, the orientation of a discretized vortex tube wake is determined using the freestream inflow conditions and a momentum-theory based induced velocity. The rotor interference velocity is a function of the freestream velocity, rotor speed, rotor disk area, rotor diameter, and rotor thrust of each rotor. Based on each wake shape, the perturbation velocities are determined at the other rotor disks.

The rotor interference model uses the Biot-Savart method to calculate segment velocities of vortex rings that represent the wake shed of a rotor [19]. The rotor wake model used in the original multirotor vehicle performance prediction method was compared to analytical results from Castles and De Leeuw and showed good agreement of induced velocities in the rotor plane using 100 vortex segments and 200 vortex rings per rotor [14.

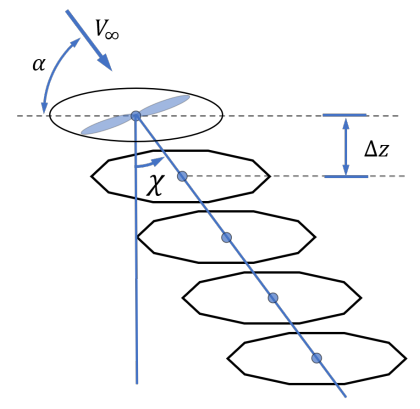

(a) Skew angle and distance between ring wakes

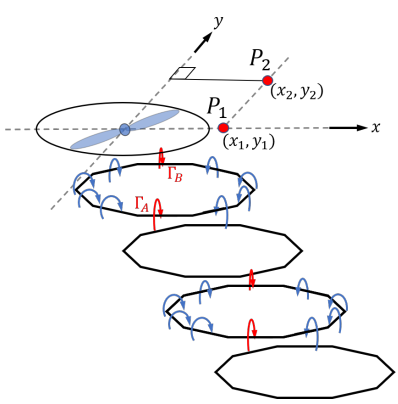

(b) Circulation about the vortex segments

Figure 3.5: Example of vortex rings of forward travelling rotor.

Figure $3.5 \mathrm{a}$ shows the inflow velocity, $V_{\infty}$, at an angle of attack, $\alpha$, applied to a rotor. The rotor has equally spaced wakes by some distance, $\Delta z$, and the centre of the vortex rings are skewed by an angle, $\chi$, from the vertical axis of the rotor plane. The skew angle is calculated as the tangent of the velocity parallel to the rotor plane, $u$, and the combination of freestream flow and the induced velocity through 
the rotor plane, $w$, as [12]:

$$
\chi=\tan ^{-1}\left(\frac{u}{w}\right)
$$

where the velocity parallel to the rotor plane is calculated as:

$$
u=V_{\infty} \cos \theta
$$

and the combined freestream and induced velocity through the rotor plane is calculated as:

$$
w=\frac{1}{2}\left(V_{\infty} \sin \theta+\sqrt{\left(V_{\infty} \sin \theta\right)^{2}+\frac{2 T}{\rho A}}\right)
$$

or,

$$
w=V_{\infty} \sin \theta-v_{\text {ind }}
$$

The induced velocity, $v_{\text {ind }}$, through the rotor plane is [22]:

$$
v_{\text {ind }}=\frac{1}{2}\left(-V_{\infty} \sin \theta+\sqrt{\left(V_{\infty} \sin \theta\right)^{2}+\frac{2 T}{\rho A}}\right)
$$

Finally, the distance between ring wake centres is calculated by an offset in the z-direction of the horizontal plane as:

$$
z=\frac{1}{n 2 B}\left(V_{\infty} \sin \theta+\sqrt{\left(V_{\infty} \sin \theta\right)^{2}+\frac{2 T}{\rho A}}\right)
$$

A correction was made to the z-offset equation in the Ref. [14 version of the wake interference model which eliminated the overlapping of the first shed wake in the same plane as the rotor.

Figure $3.5 \mathrm{~b}$ shows the constant circulation, $\Gamma$, about vortex segments within a vortex ring. The constant circulation, $\Gamma$, of the vortex segment is calculated as [22]:

$$
\Gamma=\frac{T}{\rho R^{2} n \pi B}
$$

The circulation is a function of thrust, $T$, density, $\rho$, rotor radius, $R$, rotor speed in revolutions per second, $n$, and number of blades, $B$. The velocities induced by each vortex segment onto each rotor are 
calculated based on the circulation, skew angle, and rotor wake offset, z, using the Biot-Savart based approach. For a rotor, the sum of velocities induced by the other rotor segments is the interference velocity, $v_{i n t, z_{R}}$, applied to that rotor.

\subsubsection{Wake Interference Model Limitations}

There is a limitation to the wake interference model when observing the normal velocity through the rotor plane directly outside the ring wake along the lateral axis of the rotor plane. Figure $3.5 \mathrm{~b}$ shows a two points, $P_{1}$ and $P_{2}$, that is located directly behind the rotor within the rotor plane. Each vortex segment on the vortex ring induces a velocity on $P_{1}$ and $P_{2}$. Within the bounds of the ring wake, the sum of the segments results in a downwash. An upwash exists outside the bounds of the ring wake. Along the lateral axis directly outside the rotor disk and the outside the bounds of the shed rotor wakes should only exist an upwash, as shown by the vertical induced velocity vectors to the direct left and right of the rotor disk in Fig. 2.6. The same upwash condition should also be true in forward flight, but using the ring wake model presents downwash components laterally outside the rotor disk. With increasing lateral distance from the rotor disk, the induced velocities gradually become upwash components. When using the wake interference model, the induced velocities added together for $P_{2}$ shows a downwash present directly outside the rotor disk. In reality, there only exists upwash components exist along the rotor plane and outside the bounds of the rotor wake below the rotor plane [23]. The fluctuating induced velocity results predicted using the ring wake method is a result of model limitations using individual ring wakes.

To illustrate the downwash present outside the rotor disk within the rotor plane when velocities are predicted using the ring wake model, Fig. 3.6 shows the normal components of the induced velocity estimated along the lateral span of the rotor plane. The lateral span is between $-0.6<y<0.6$ at seven different positions along the longitudinal axis: $\mathrm{x}=-0.6 \mathrm{~m}, \mathrm{x}=-0.4 \mathrm{~m}, \mathrm{x}=-0.2 \mathrm{~m}, \mathrm{x}=0.0 \mathrm{~m}, \mathrm{x}=0.2$ $\mathrm{m}, \mathrm{x}=0.4 \mathrm{~m}$, and $\mathrm{x}=0.6 \mathrm{~m}$. The rotor grid is shown in Fig. 3.7. The chosen propeller parameters are listed in Tab. 3.1 and are of a single T-Motor 18x6.1 rotor in steady level flight travelling at $10 \mathrm{~m} / \mathrm{s}$ at an angle of attack of zero degrees. 


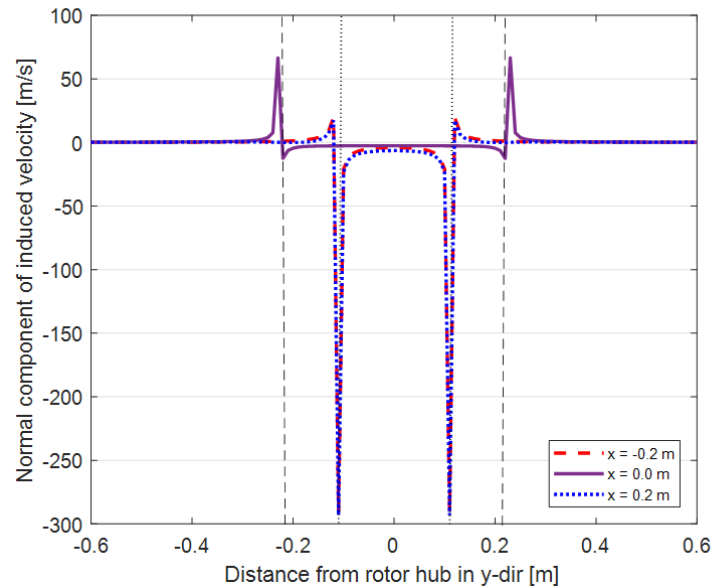

(a) $\mathrm{x}=-0.2 \mathrm{~m}, \mathrm{x}=0.0 \mathrm{~m}, \mathrm{x}=0.2$

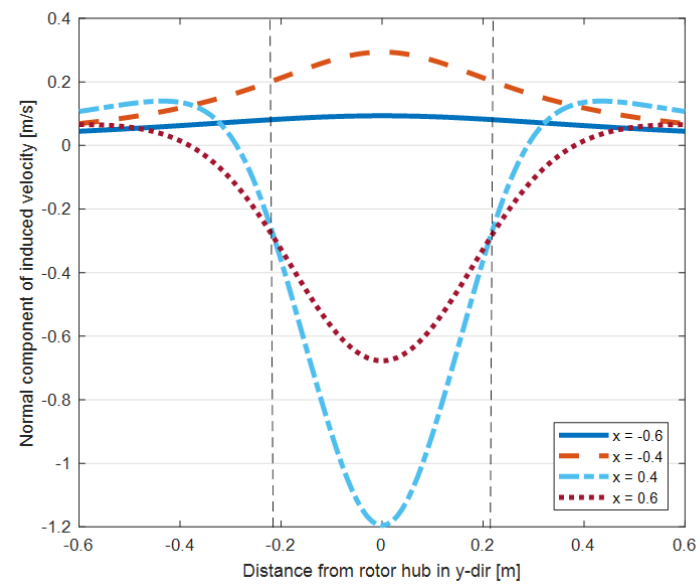

(b) $\mathrm{x}=-0.6 \mathrm{~m}, \mathrm{x}=-0.4 \mathrm{~m}, \mathrm{x}=0.4, \mathrm{x}=0.6$

Figure 3.6: Normal components of induced velocity through seven lateral sections of the rotor plane between $-0.6<x<0.6$.

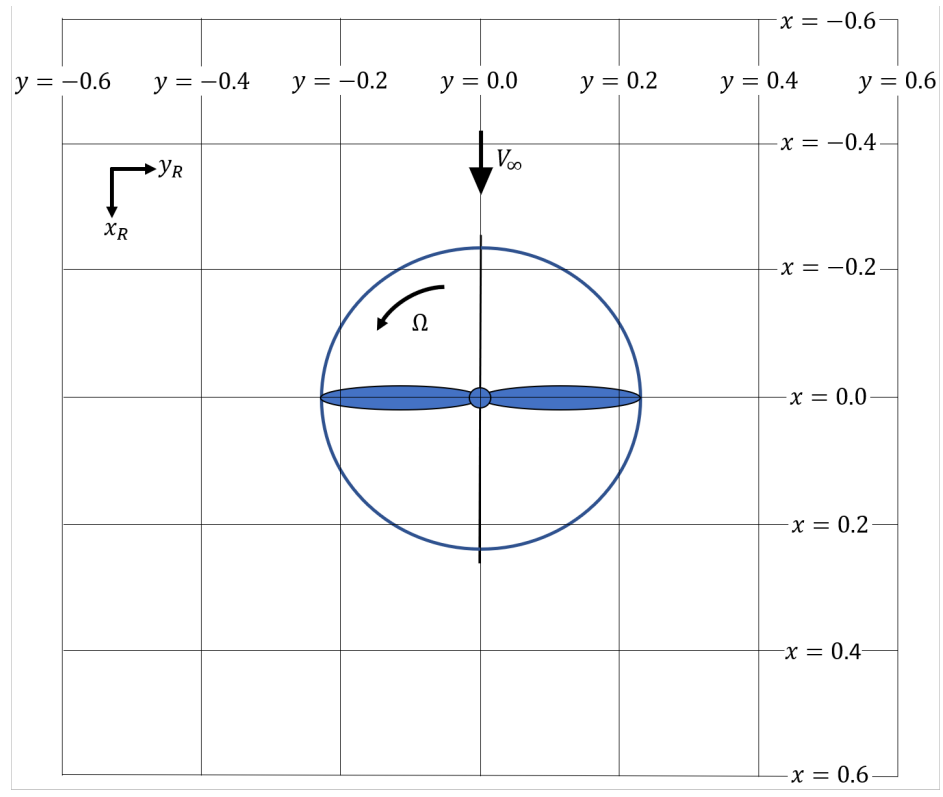

Figure 3.7: Normal components of induced velocity through seven lateral sections of the rotor plane between $-0.6<x<0.6$.

Table 3.1: Wake interference model test input variables. 


\begin{tabular}{|l|l|}
\hline Density $\left(\mathrm{kg} / \mathrm{m}^{3}\right)$ & 1.225 \\
\hline Number of blades & 2 \\
\hline Number of segments & 100 \\
\hline Number of wakes & 200 \\
\hline Rotor diameter $(\mathrm{m})$ & 0.4572 \\
\hline Freesteam velocity $(\mathrm{m} / \mathrm{s})$ & 10 \\
\hline Rotor thrust $(\mathrm{N})$ & 8.6 \\
\hline Rotor speed $(\mathrm{RPM})$ & 2100 \\
\hline Rotor pitch $(\mathrm{deg})$ & 11 \\
\hline
\end{tabular}

Figure $3.6 \mathrm{a}$ shows results for the induced velocity within the bounds of the rotor during forward flight. Figure $3.6 \mathrm{~b}$ shows results for induced velocity in front and aft of the same rotor during forward flight. The large spikes in Fig. 3.6a are a result of the singularities of induced velocities at the edge of the rotor area. The vertical dashed lines represent the edge of the rotor within the y-axis through the origin of the rotor. The dotted vertical lines represent the edge of the rotor at an offset of $0.2 \mathrm{~m}$ from the x-axis. Within the bounds of the dashed lines and the dotted lines, the negative induced velocity of all trends represent downwash that exists at the rotor disk. The area of interest to consider, when observing the limitations to the ring wake model, is adjacent to the vertical dashed lines in Fig. 3.6b.

Directly outside the vertical lines, or the bounds of the rotor disk, are still downwash components present as a result of the sum of induced velocities estimated by the rotor wake model. In reality, there are no downwash velocities outside the bounds of the ring wakes. This is a result of the discrete nature of the ring vortices that represent a continuous rotor wake.

Other wake analysis methods, such as those outlined in Ref. [13], provides results where the downwash issue is eliminated. Ref. [18] presented results of a cylindrical wake method showing that the flow field outside the wake bounds only had upwash components while the flow within the wake bounds had only downwash components. Other wake models, however, were not compared within the scope of this thesis.

The magnitude of interference velocity compared to the freestream contributions are small, as will be explored in Chapter 5. So for the purposes of using a computationally fast wake model within the performance prediction method outweighs the limitations of the current wake interfere model that is used. 


\subsubsection{Fuselage Interference}

Typical components of a multirotor vehicle fuselage, such as the ones shown in Fig. 3.3 , include solid bodies that generate minimal or no lift. Having these bodies in close proximity to the rotors causes a disruption of the flowfield at the rotors. Adding the freestream-fuselage interactions, referred to as fuselage interference velocity, to the freestream velocity better captures the complex flowfield about a multirotor vehicle.

The body interference depends on the freestream velocity, vehicle pitch, and the distance between the rotor hubs and the body surface. Figure 3.8 shows the velocity components applied to two points, $P_{1}$ and $P_{3}$, that represent positions of the lead and rear rotors of a quadrotor flying in diamond configuration, similar to the rotor positions shown in Fig. 2.8 .

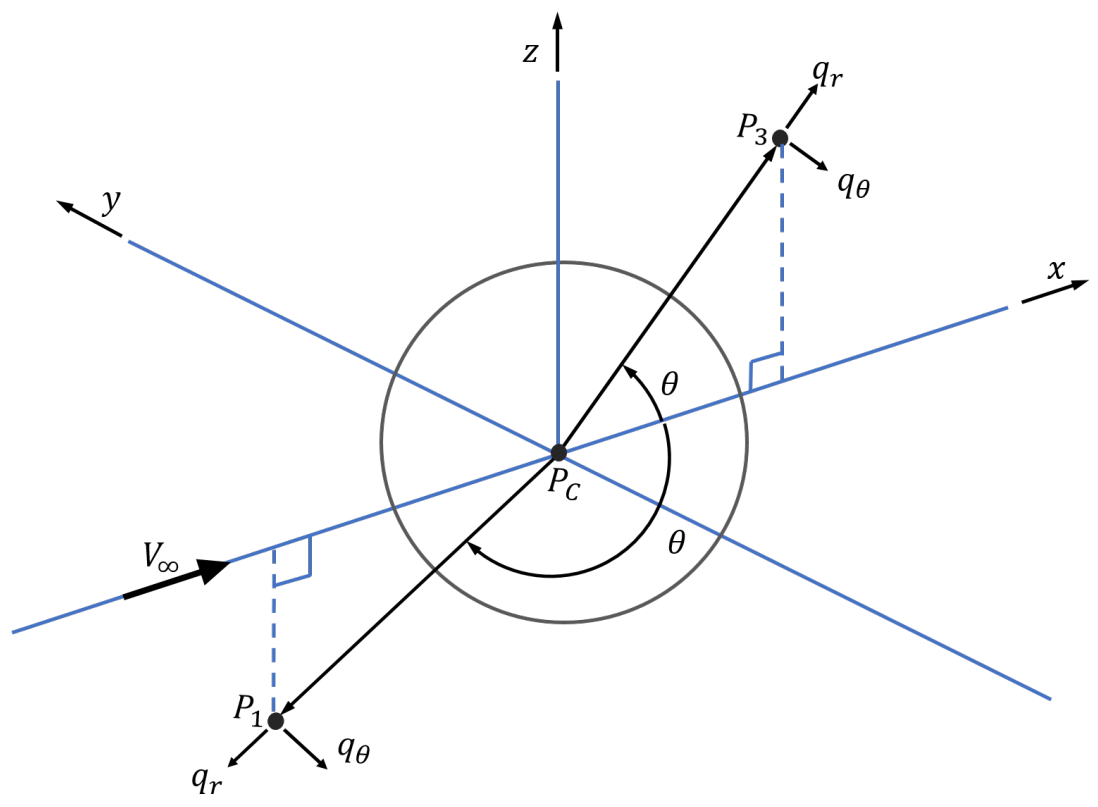

Figure 3.8: Radial and tangential flow components applied to points $P_{1}$ and $P_{3}$.

To obtain the interference velocities caused by the flow about the body onto each of the rotors, the $q_{r}$ and $q_{\theta}$ are converted into Cartesian coordinates velocity components and subtracted from the freestream velocity components. The flowfield components are determined using the potential flow equations for 
the flow about a sphere [19]:

$$
q_{r}=V_{\infty} \cos \theta\left(1-R^{3} / r^{3}\right)
$$

and

$$
q_{\theta}=-V_{\infty} \sin \theta\left(1+R^{3} /\left(2 r^{3}\right)\right)
$$

where $q_{r}$ and $q_{\theta}$ are the radial and tangential velocities in the flow field about a sphere of radius, $R$. Since the multirotor vehicle performance model operates in longitudinal horizontal flight, $\theta$ in Eqs. 3.21 and 3.22 refer to the angle between the horizontal and the vector, $r$ between the body centre and the rotor hub in global coordinates. This model ignores any flow disturbances due to any circulation generated by the lifting fuselage, which should be relatively small compared to the flow due to the flow displacement due to the body.

\subsection{Interference Model}

The interference model is used in the performance prediction method to predict the rotor speeds for each rotor to maintain a given thrust. Figure 3.9 shows the incorporation of the fuselage and rotor interference models into an algorithm that establishes the resultant inflow conditions to predict the rotor speed requirements for each rotor. After the force trim model establishes the vehicle pitch and thrust requirement, the fuselage interference model to predict the fuselage interference, $v_{b o d y}$, using Eqs. 3.21 and 3.22 ,

The rotor interference model inputs are thrust, vehicle pitch, rotor speed, and fuselage interference predictions that predict the resultant inflow conditions and rotor speeds for each rotor. The initial rotor speed prediction is established from the force trim model. The rotor interference velocity, $v_{\text {int }}$, for each rotor is calculated using the ring wake interference module. The resultant inflow velocities and angles are calculated using Eqs. 3.12 and 3.13, respectively. Using the updated inflow conditions and rotor thrust values, updated rotor speeds, rotor inplane hub forces, $F_{x}$ and $F_{x}$, rotor hub moments, $M_{x}, M_{y}$ and torque $Q$, are determined using the rotor performance lookup tables. The rotor interference model iterates between initial and final rotor speeds. The rotor interference model is typically converges after about five iterations. 


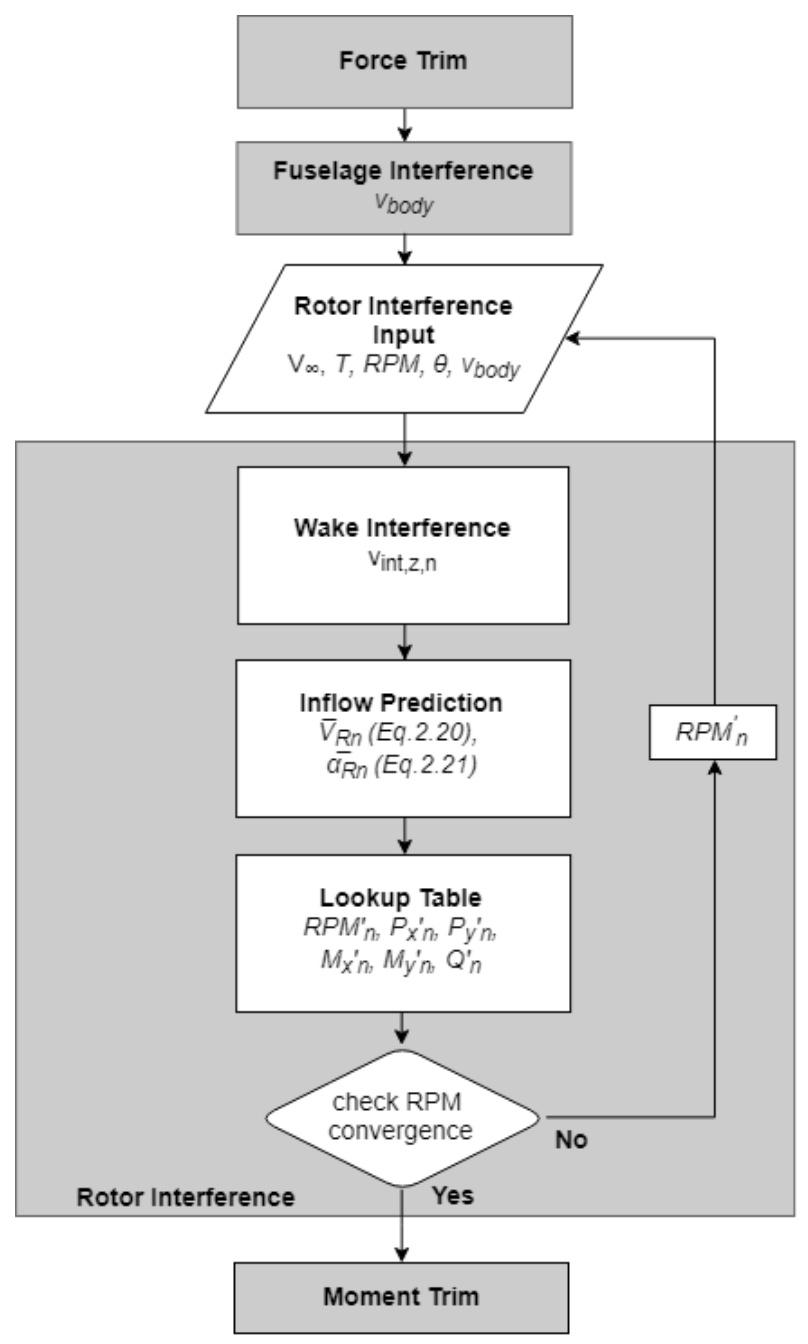

Figure 3.9: Rotor speed prediction algorithm.

\subsection{Power Prediction}

In the original multirotor vehicle performance method of Ref. 14, the total vehicle power was predicted as the sum of rotor power, parasitic power, and interference power. Rotor power is a function of freestream velocity, rotor speed, air density, and rotor geometry as shown in Eq. 2.7. Parasitic power is a function of the parasitic drag of each component and the freestream velocity. Interference power is due to the rotor interference velocities that the rotors experience. Using the interference model to predict rotor speeds eliminates the need to calculate the change in induced power due to interference effects separately. This 
rotor power reflects the inflow conditions of the freestream and interference velocities. The total rotor power, $P_{t o t}$, used in the multirotor vehicle performance model is defined as:

$$
P_{t o t}=P_{r o t}+P_{p a r}
$$

where the rotor power $P_{r o t}$ is the rotor power, and $P_{\text {par }}$ is the parasitic power. The rotor power is the sum of profile, induced, and interference power.

\subsection{Moment Trim}

The moment trim model described in this section looks to resolve the residual moments, for example as shown in Fig. 2.10a, on the vehicle to zero as done in Fig. 2.10b. Residual moments are the moments due to the forces of the vehicle that act at a moment arm about the vehicle reference point. Generally the vehicle reference point the point where all support arms intersect. The total residual moment, $M_{(x, y, z)}$, is the sum of the moments produced by each vehicle component. The total residual moment of the vehicle is calculated as:

$$
M_{(x, y, z)}=\Sigma\left(\vec{r}_{i} \times \vec{F}_{i}\right)+\Sigma \vec{M}_{\text {Rotors }}
$$

where $M_{(x, y, z)}$ is the sum of the cross product between the component moment arm, $\vec{r}_{i}$, and the force

component, $\vec{F}_{i}$, plus the sum of the aerodynamic hub moments of the rotors. The force components included in the moment model are the rotor thrusts, the rotor inplane forces, and the induced drag and lift of the fuselage, the drag forces calculated from the parasitic drag model, and the weight of each of the vehicle components. Hub moments are the rolling and pitching moments, and torques of each rotor as predicted from the rotor interference model.

To resolve the total residual moments to zero for a vehicle in a one-leading rotor configuration, the updated rotor thrust forces of the lead and rear rotors are calculated by subtracting and adding, respectively, half of the total residual pitching moment, $M_{y}$, divided by the moment arm, $R$. Lead and rear rotor thrusts are calculated using:

$$
T_{\text {lead,new }}=T_{\text {lead }}-\frac{M_{y}}{2 R}
$$


and

$$
T_{\text {rear, new }}=T_{\text {rear }}+\frac{M_{y}}{2 R}
$$

For a two-leading rotor configuration, the total moment is divided by four to calculate the change in thrust for each rotor. Once new rotor thrusts are determined, the rotor interaction model is repeated to determine the updated rotor forces, rotor moments, rotor speeds, and rotor power based on new thrust and inflow conditions. The rotor interaction and moment trim models are repeated until the total vehicle moment reaches below a near zero threshold of $0.0001 \mathrm{Nm}$.

The following section shows the implementation of the modules within in the multirotor vehicle performance model used to determine vehicle performance over a range of airspeeds.

\subsection{Prediction Model Algorithm}

Figure 3.10 shows the algorithm that makes up the multirotor vehicle performance method. The algorithm uses user inputs establishing air properties, vehicle altitude, component geometries, component masses, and an input range of vehicle speeds.

The method input setup time is relatively fast. The user specifies the rotor type used on the vehicle and provides the associated rotor performance tables. The input files used in this report are in Appendix A and require basic component geometries, component masses, airspeed range, environment conditions, and rotor performance file input. Creating a computer generated model is not required to give detailed geometries to the input file saving setup time. Ideally, rotor performance tables can be collected in a database for quick model input.

There are six main models in the multirotor performance prediction method: the parasitic drag model, the force trim model, the fuselage interference model, the rotor interference model, the moment trim model, and the power prediction model. The following list outlines the steps between the six models of the algorithm:

1. Starting from the first airspeed set in the input airspeed range, the algorithm predicts fuselage parasitic drag, $D_{\text {par }}$, that is associated with the vehicle geometries and rotor speeds.

2. Using the total parasitic drag, freestream velocity, and vehicle weight, the force trim model iterates between initial and final vehicle pitch, $\theta$, results to determine force trim conditions. Force trim 
conditions include calculating the induced drag and lift of the central body, $D_{\text {ind,body }}$ and $L_{b o d y}$, using Eqs. 3.10 and 3.8 respectively, the rotor thrust, Eq. 3.6, and pitch attitude, Eq. 3.5. An initial rotor speed prediction is determined using the rotor performance lookup table. Only two iterations are typically required of the force trim model for the pitch angle to meet the convergence threshold.

3. Using the vehicle pitch result, the fuselage interference, $v_{\text {body }}$, is calculated using Eqs. 3.21, 3.22 and the radius of the central body.

4. The thrust, pitch, rotor speed, and fuselage interference predictions are used to predict new inflow velocities and angles using Eqs. 3.12 and 3.13 , respectively. The rotor interference velocities, $v_{\text {int }}$, for each rotor are calculated using the ring wake interference module. Using the updated inflow conditions and rotor thrust values, the final rotor speeds, rotor inplane hub forces, $F_{x}$ and $F_{x}$, rotor hub moments, $M_{x}, M_{y}$ and torque $Q$, are determined using the performance lookup table. The rotor interference model iterates between initial and final rotor speeds until convergence is reached.

5. The moment trim model uses the rotor forces and hub moments, component weights, parasitic drag, and fuselage induced drag and lift to calculate vehicle moments, $M_{t o t a l, x}, M_{t o t a l, y}$ and $M_{t o t a l, z}$. The moment trim module then determines a thrust new distribution between lead and rear rotors using Eqs. 3.25 and 3.26 respectively. The rotor speeds, rotor forces, and rotor hub moments of the lead and rear rotors are then updated using the lookup table scheme. Since the rotor thrusts and rotor speeds are updated, the rotor interactions will change the interference velocities of each rotor. This requires an iteration back to the rotor interference model using updated rotor thrust and speeds. The moment trim model typically eight iterations to meet the convergence threshold.

6. The power prediction model uses the rotor power and parasitic power to predict the total vehicle power, Eq. 3.23 .

After determining the operation values for one airspeed, the performance model advances to the next airspeed, $V_{\infty}+\Delta V$.

The multirotor vehicle performance model predicts thrust requirements to maintain trim at each airspeed between $0-30 \mathrm{~m} / \mathrm{s}$ in under five minutes using a standard laptop Intel ${ }^{\circledR} \mathrm{Core}^{\mathrm{TM}} \mathrm{i}$ processor. 
The following studies in Chapters 4, 5, and 6 use this prediction method algorithm, or a modified version of the algorithm. 


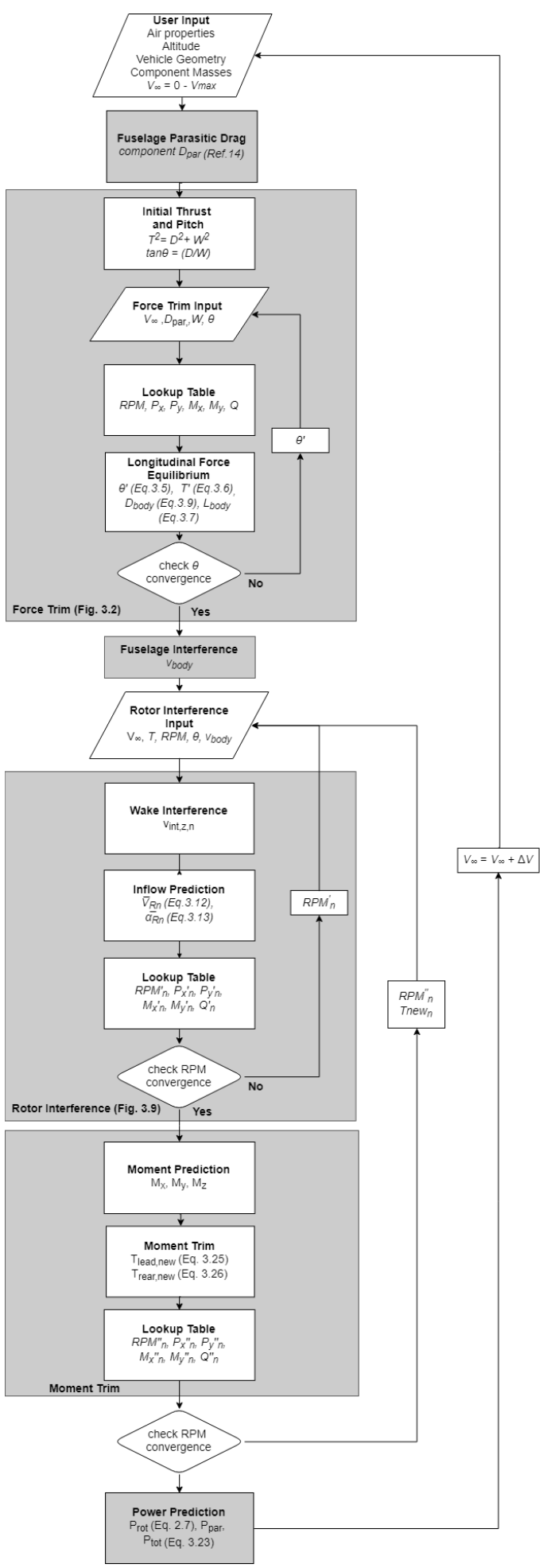

Figure 3.10: Implementation of multirotor vehicle performance model. 


\section{CHAPTER 4}

\section{COMPARISON OF PREDICTION}

\section{AND EXPERIMENTAL WIND \\ TUNNEL AND FLIGHT TEST}

\section{RESULTS}

In this chapter, two comparitive tests cases are discussed. The first case compares wind tunnel results of a 3DR Solo, a commercially available quadrotor vehicle, and to multirotor performance predictions. The experimental data are from wind tunnel tests at NASA Ames [20]. Data includes rotor and vehicle lift, drag and vehicle pitching moment. The multirotor vehicle performance prediction model was modified in order to reflect determining the lift and drag of a vehicle over a range of pitch attitudes at a constant freestream velocity. In addition to the comparison with data from Ref. 20, the second comparison study was conducted with flight test data of the Aeryon SkyRanger. The recorded data includes rotor speeds, vehicle power, and vehicle pitch at points of steady state flight. 


\subsection{DR Solo Performance}

In 2016 a group from NASA Ames performed wind tunnel tests using five commercially available multirotor vehicles: 3DR Solo, 3DR Iris+, DJI Phantom 3 Advanced, Drone America DAx8, and SUI Endurance [20]. The researchers identified a lack of published performance data of multirotor vehicles that is suitable for the validation of performance prediction methods. The current analysis compared the data published by NASA and the performance results that were generated using the multirotor performance prediction method.

\subsubsection{Revised Prediction Method}

In order to predict the vehicle pitching moment using set rotor speeds, the table lookup scheme that is used to determine the rotor performance was modified in order to allow to input rotor speed, dynamic pressure, and inflow angle and to output forces and moments of the rotor. Figure 4.1 shows the modified multirotor vehicle performance model that was used to predict moments over a range of pitch values. The comparison model uses the fuselage drag, body interference, rotor interference, and moment models to predict rotor thrust, vehicle lift, vehicle drag, and vehicle pitching moment of the 3DR Solo.

The user input file for the performance comparison method includes atmospheric properties, rotor geometry, vehicle component geometry, and information about the component mass. A sample input file for the 3DR Solo is given in Appendix A. Unknown geometric parameters were obtained by scaling figures of the 3DR Solo vehicle using known rotor hub and height parameters [24. Figure 4.2 shows the top and side view images of the 3DR vehicle used to scale component geometries. It has an overall diameter of $0.46 \mathrm{~m}$ and a height of $0.25 \mathrm{~m}$. Its four rotor are of $0.254 \mathrm{~m}$, or 10-inches, diameter. 


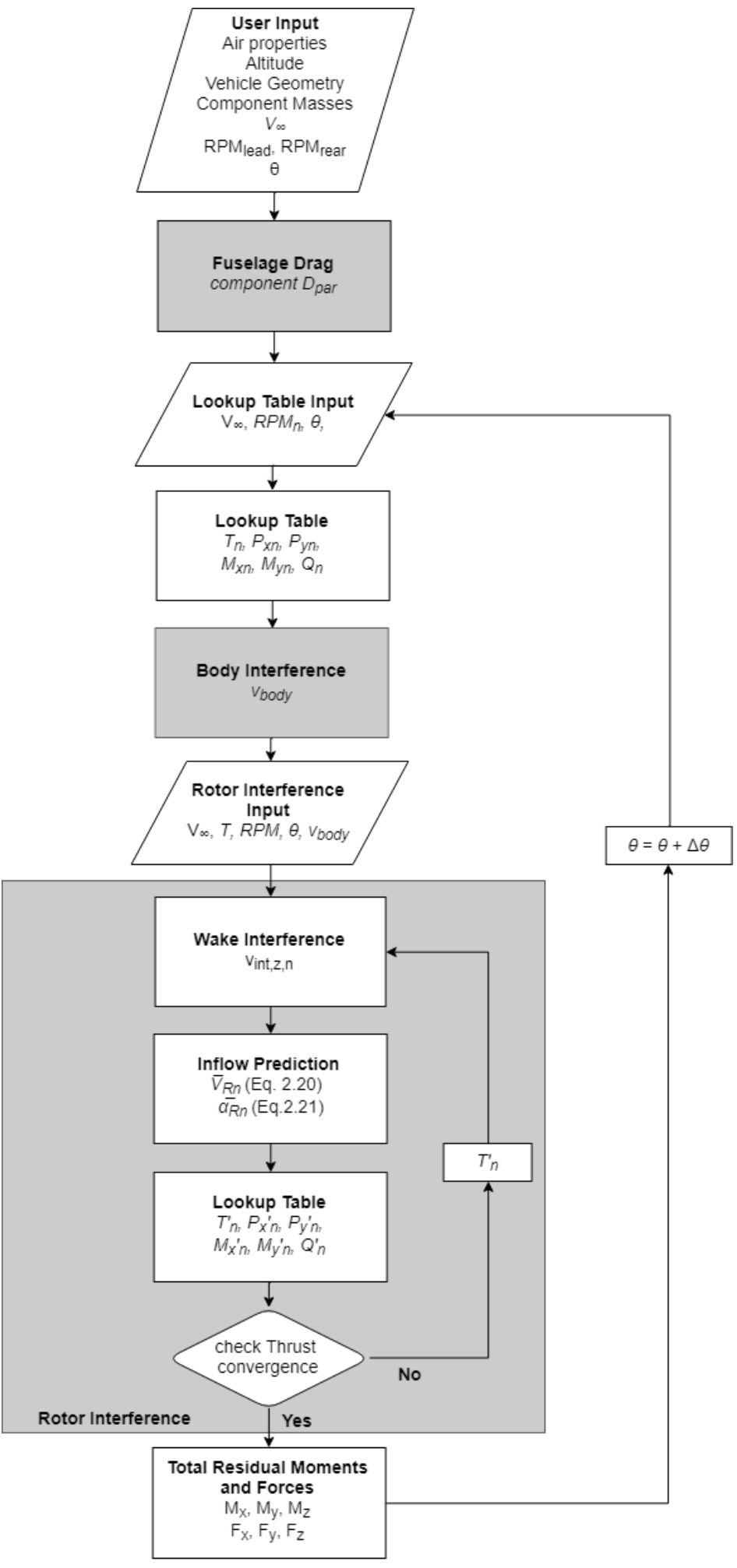

Figure 4.1: Performance comparison method algorithm. 


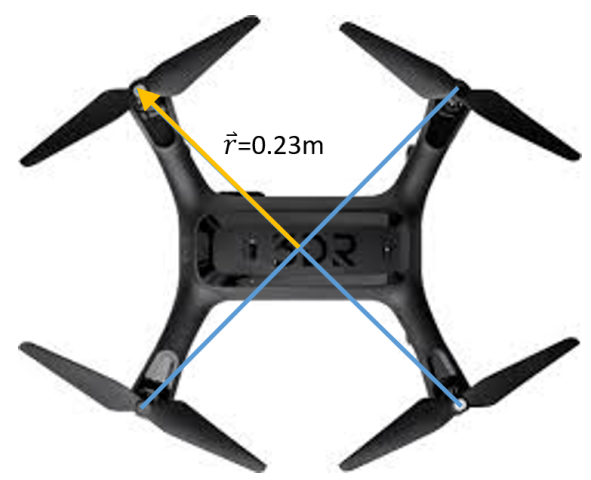

(a) Top view 24 .

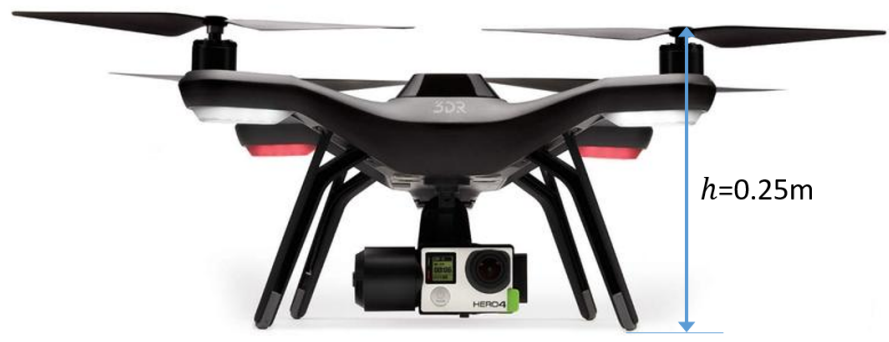

(b) Front view [25].

Figure 4.2: Reference rotor hub radius and height for 3DR Solo vehicle.

\subsubsection{Rotor Lift and Drag}

In order to use the prediction method, rotor performance tables were required. Without having access to the geometry of the 3DR Solo $10 \mathrm{x} 4.5$ propeller used in the wind tunnel testing, a GCRSP 10x8 rotor, whose geometry is published in the University of Illinois propeller database, was selected as a rotor performance substitute for the 3DR Solo propeller [26]. The GCRSP rotor was selected for its similar planforms to the 3DR Solo propeller, as shown in Figure 4.3. Its predicted performance shows lift and drag results that are similar to the 3DR Solo isolated rotor wind tunnel measurements of Ref. 20. Rotor prediction data were generated using the blade element moment theory model used in Ref. 17, In order to match the thrust results between prediction and measured rotor data, the pitch of the GRCSP 10x8 rotor was corrected form $8^{\circ}$ to $4.5^{\circ}$ to have the same pitch angle as the $3 \mathrm{DR}$ Solo $10 \mathrm{x} 4.5$ rotor.

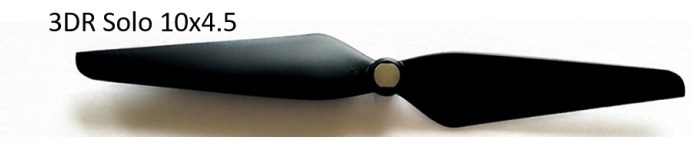

(a) 3DR propeller 10x4.5 [27].

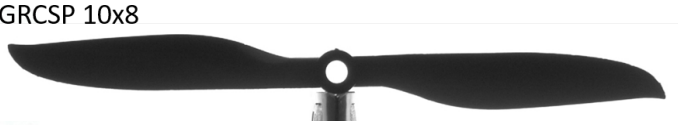

(b) GRCSP 10x8 [28].

Figure 4.3: Rotor types compared in rotor performance comparison.

Figure 4.4 shows isolated rotor lift and drag results from the prediction and the wind tunnel results of Ref. 20 at 6300 and 6800 RPM over a pitch angle range of $-40^{\circ}-40^{\circ}$. 


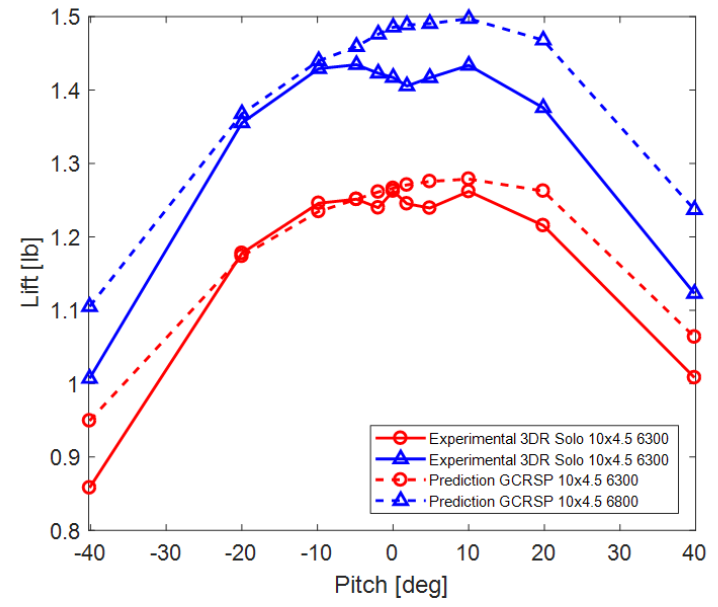

(a) Rotor lift

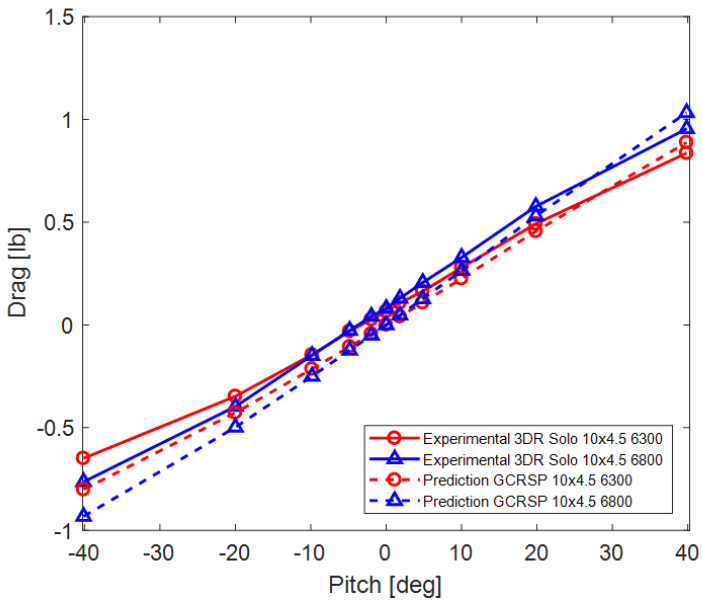

(b) Rotor drag

Figure 4.4: Rotor performance comparison between 3DR Solo 10x4.5 wind tunnel results and GRCSP $10 \times 4.5$ prediction results.

The rotor drag prediction shows an underprediction of less than $20 \%$ to the wind tunnel results but a similar cross-over trend around between $-8^{\circ}$ and $-2^{\circ}$ pitch for both rotor speeds. The rotor lift prediction shows similar trends for the $6300 \mathrm{RPM}$ case at pitch angles between $0^{\circ}$ and $-20^{\circ}$, which is the typical range for small quadrotors in steady, level forward flight. Overall, general trends are similar for lift results. There is a positive slope at negative pitch angles under $-10^{\circ}$ and a negative slope for positive pitch angles after $+10^{\circ}$ which meet at peak lift between $-10^{\circ}$ and $+10^{\circ}$. Larger deviations of lift are seen around edgewise flight $\left(\alpha=0^{\circ}\right)$ for the higher rotor speed case, within about $10 \%$ overprediction of lift wind tunnel results. Since the original 3DR Solo rotor could not be used in this comparative test, the performance results of the modified GRCSP 10x8 rotor data shows to have similar trends within about $10 \%$ to the 3DR Solo rotor to justify the use of the rotor method.

\subsubsection{Vehicle Lift and Drag}

Using the modified GCRSP 10x8 rotor performance tables, the vehicle lift and drag predictions of a four-rotor 3DR Solo vehicle were generated using the approach outlined in Fig. 4.1. The subsequent prediction results and wind tunnel results are shown in Fig. 4.5 for the full vehicle with all four rotors have the same rotational speeds. The freestream velocity was held constant. 
The prediction overpredicts lift results at higher rotor speeds, particularly around $\alpha=0^{\circ}$. At lower rotor speed cases of 4600 and 5100 RPM, the experimental and prediction results for lift show similar trends with similar lift results around $0^{\circ}$ pitch. As the rotor speeds increase, there is an increasing overprediction in lift around $0^{\circ}$ pitch. The overpredictions at higher rotor speeds can be attributed to the pitch correction that was used when generating the rotor performance tables using the GRCSP 10x8 rotor. As observed in Fig. 4.4, overpredictions in lift exist in the isolated rotor tests for the higher rotor speed cases. The same rotor lift results are shown to carry over in the overpredicted vehicle lift results. Using the exact geometries and airfoil type used for the 3DR Solo model are likely to improve the accuracy of the prediction results.

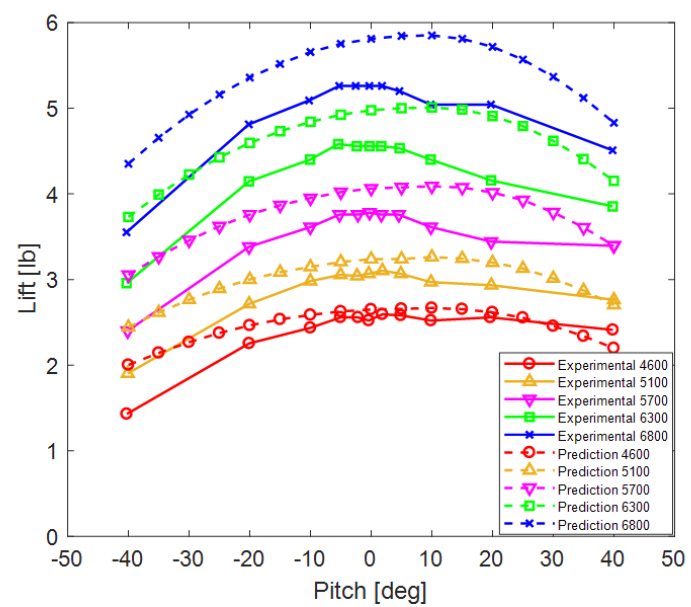

(a) Vehicle lift

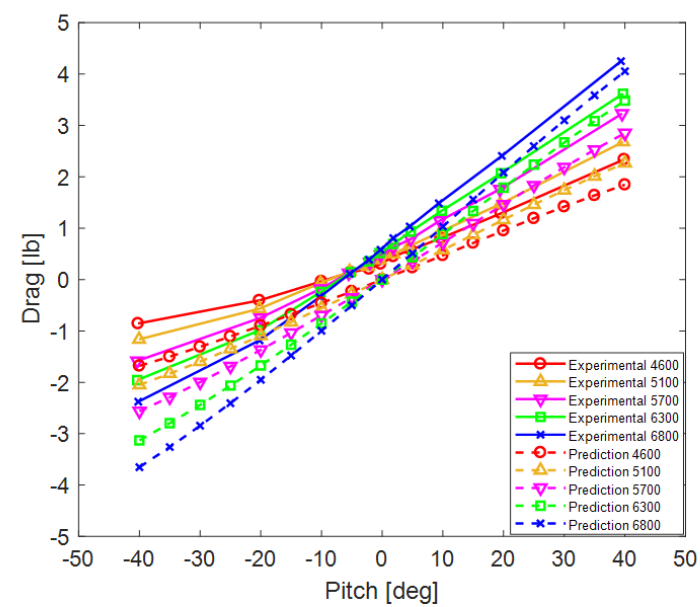

(b) Vehicle drag

Figure 4.5: 3DR Solo vehicle experimental and prediction lift and drag results with equal rotor speeds.

Drag was also overpredicted for all cases with the closest agreement to the experimental results around $0^{\circ}$ pitch and an increasing deviation from the wind tunnel results with increasing or decreasing pitch. Nevertheless, the drag predictions show similar slopes between prediction and wind tunnel results where all trends cross around $0^{\circ}$ for the prediction results and around $5^{\circ}$ for the wind tunnel results. It is assumed that the drag predictions are due to the parasitic drag module being dependent only on freestream flow and not angle of attack. The model does not account for the cross-sectional area of each component, as the angle of attack of the vehicle changes. 


\subsubsection{Full Vehicle}

Figure 4.6 shows experimental and prediction results for the pitching moment of the 3DR Solo vehicle. The predicted pitching moment results are calculated using the modified performance comparison method, Fig. 4.1, for a range of pitch attitudes, $\theta$, and at a constant dynamic pressure of $0.48 \mathrm{lb} / \mathrm{ft}^{2}$. The predicted and experimental results exhibit similar slopes with respect to vehicle pitch attitudes. However, the predicted pitching moments are underpredicted by about 5-7 lb-in consistently from the experimental results. The offset between experimental and prediction trends is a result from the thrust offset between the experimental and predicted vehicle results shown in Fig. 4.5. Despite these differences that can be attributed to difference between GRCSP and 3DR Solo rotor geometry, the prediction captures a similar behaviour in longitudinal stability as observed in the experiment.

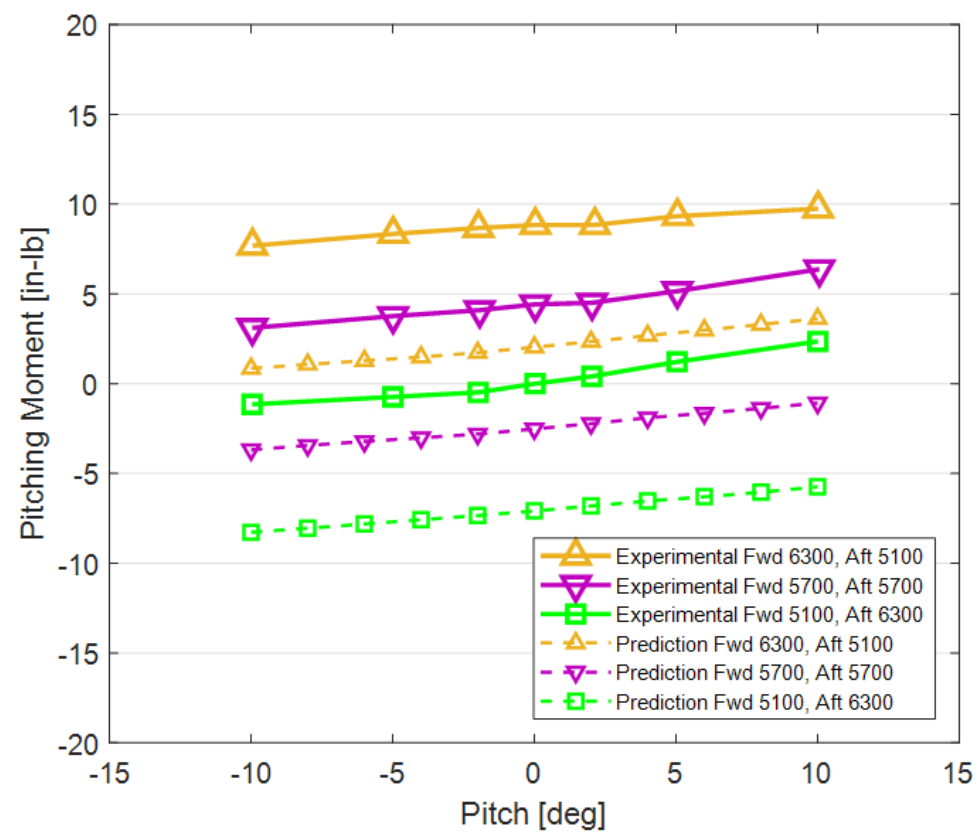

Figure 4.6: Experimental [20] and prediction of pitching moment of 3DR Solo vehicle.

The pitching moment slopes in Fig 4.6 shows the inherent instability of quadrotors. For a vehicle with constant rotor speeds, with increasing pitch angle the pitching moment of the vehicle also increases. The multirotor vehicle performance model is a useful tool to predict these pitching moment slopes to capture the stability trends of a vehicle. 


\section{$4.2 \quad$ Flight Test Results}

In order to further validate the performance of the prediction model, flight testing was performed using an Aeryon SkyRanger at steady level flight over a range of airspeeds. Rotor speeds, vehicle power, and pitch attitude flight test data were provided by Aeryon Labs Inc. During testing, the Aeryon SkyRanger had no camera payload attached and used a 15-inch proprietary rotor. The total vehicle weight was 2.6 kg. Atmospheric conditions present during the day of testing are summarized in Table 4.1

Table 4.1: Atmospheric conditions during SkyRanger flight testing

\begin{tabular}{|l|c|}
\hline Altitude $(\mathrm{m})$ & 350 \\
\hline Temperature $\left({ }^{\circ} \mathrm{C}\right)$ & 8 \\
\hline Wind $(\mathrm{km} / \mathrm{hr})$ & 13 West \\
\hline Pressure $(\mathrm{kPa})$ & 101.2 \\
\hline Density $\left(\mathrm{kg} / \mathrm{m}^{3}\right)$ & 1.25 \\
\hline
\end{tabular}

The vehicle was set to fly back and forth in an East-West direction at groundspeeds of 3, 5, 8, and $10 \mathrm{~m} / \mathrm{s}$. The vehicle travelled six legs, three to the East and three to the West, for 30 seconds each in order to ensure the vehicle had reached steady state at each target speed. Limits to groundspeed and acceleration fluctuation were applied in order to filter the flight test data. These limits are listed in Table 4.2. Steady state criteria required that the measured longitudinal acceleration was less than a magnitude of $0.4 \mathrm{~m} / \mathrm{s}^{2}$ and groundspeed to be within $0.2 \mathrm{~m} / \mathrm{s}$ for a minimum of 5 seconds. Postprocessing was performed by Aeryon Labs Inc. by including the wind speed to the measured ground speeds. The corresponding rotor speeds, power, and pitch data associated to the steady state time sets were averaged. For any plot, error bars of the flight test points indicate the range of standard deviation of the experimental data sets.

Table 4.2: Steady state criteria for flight test data.

\begin{tabular}{|l|c|}
\hline Acceleration $\left(\mathrm{m} / \mathrm{s}^{2}\right)$ & $+/-0.4$ \\
\hline Groundspeed $(\mathrm{m} / \mathrm{s})$ & $+/-0.2$ \\
\hline Elapsed time $(\mathrm{s})$ & 5 \\
\hline
\end{tabular}


Figure $4.7 \mathrm{a}$ shows a of rotor speeds that were measured during flight test and predicted. The figure shows the rotor speed difference referenced to the predicted rotor speed at hover in order to preserve the proprietary performance information regarding the SkyRanger and its rotor. The prediction results were generated for rotor speeds, total power, and pitch attitude using the multirotor performance prediction method.

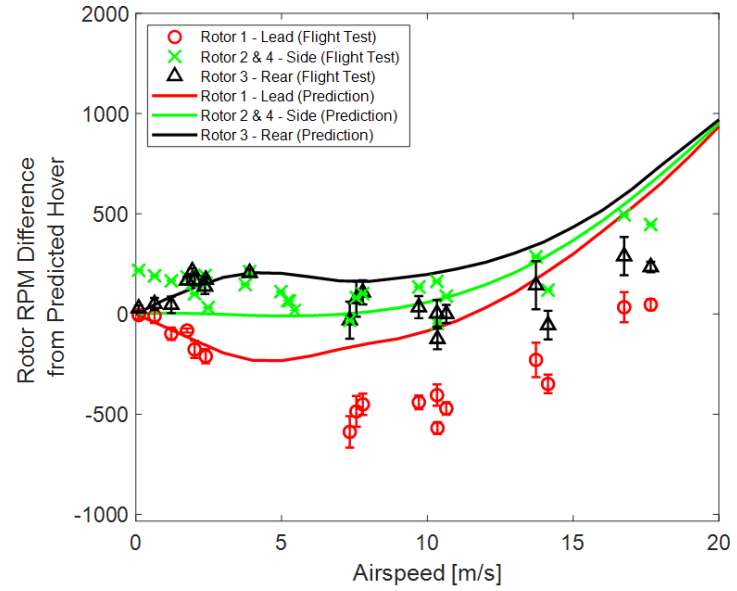

(a) Difference between RPM at hover and RPM.

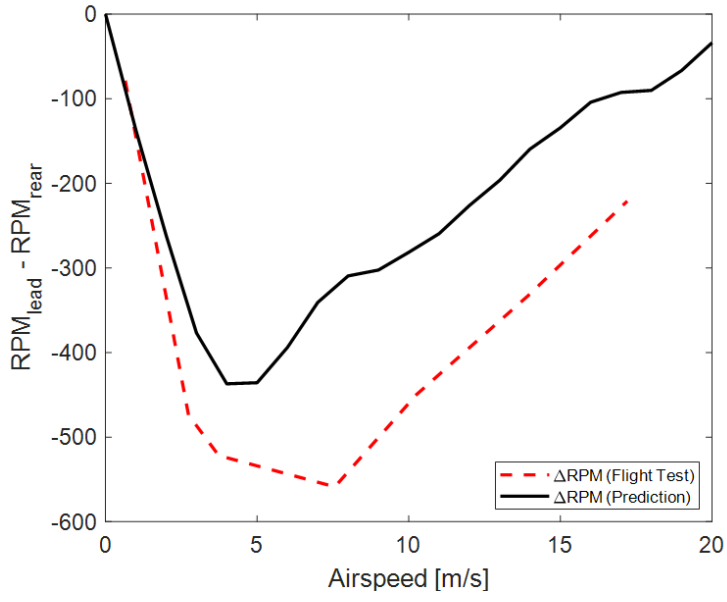

(b) Difference between lead and rear rotor speeds.

Figure 4.7: Rotor speed comparison between flight test and prediction data of a SkyRanger vehicle.

The lead and rear rotor speeds show overlapping flight test and prediction results under $5 \mathrm{~m} / \mathrm{s}$. At hover, the flight test and prediction results for lead and rear rotors are essentially equal. With increasing flight speed, the predicted results for lead and rear rotor speeds closely resembles those of the flight test results. The lead rotor has a reduction in rotor speed from hover and the rear rotor has an increase in rotor speed from hover. Faster than $5 \mathrm{~m} / \mathrm{s}$, the predicted rotor speed of the lead rotor begins to increase where the flight test results do not begin to reflect the same increase in rotor speed until around $10 \mathrm{~m} / \mathrm{s}$. The lead and rear rotor speeds after $5 \mathrm{~m} / \mathrm{s}$ are generally overpredicted by $100-200$ RPM. However, the difference between predicted lead and rear rotor speeds and flight test rotor speeds show many similar characteristics.

Figure $4.7 \mathrm{~b}$ shows the difference between in front and rear rotor rotational speeds for flight test and prediction. Even with the overpredicted rotor speed results, generally, the lead and rear rotor speeds 
have their greatest rotor speed difference between 5-7 m/s. At higher speeds, rotor speeds continuously converge. The difference between lead and rear rotor speeds reflects the thrust distribution set by the moment trim model in the performance prediction method. The flight test results reflect a similar rotor speed distribution.

The performance prediction model does not capture the side rotor performance nearly as well as the lead and rear rotors. Even though, during the flight test, the flight path was set for the vehicle to fly directly into a headwind and tailwind travelling in an East-West pattern, a crosswind component could have caused variation in side rotor speed to maintain steady level flight. The performance prediction model does not trim rolling and yawing moments.

Figure 4.8 shows the difference in total vehicle power from the flight test hover condition. Here, there is also a clear underprediction of almost $100 \mathrm{~W}$ in vehicle power at hover; However, the power measured from the SkyRanger is also a combination of electrical and mechanical power provided to the vehicle. The multirotor vehicle performance model only provides estimates of total vehicle power as a sum of rotor and parasitic power.

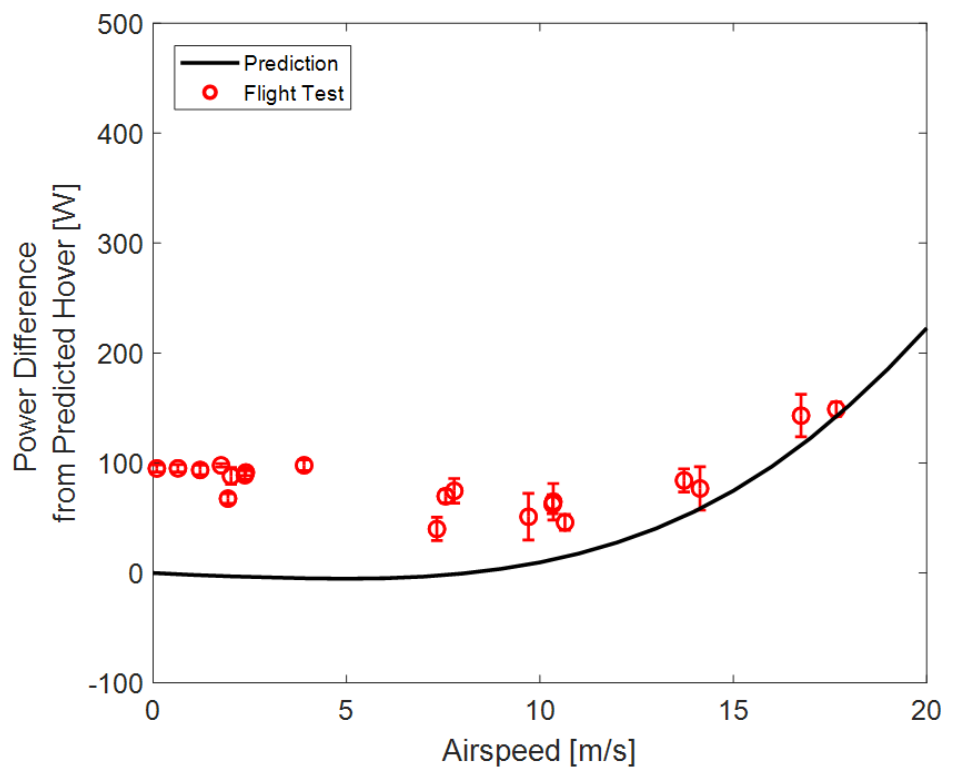

Figure 4.8: Vehicle power comparison between flight test and prediction data of a SkyRanger vehicle.

Finally, Fig. 4.9 compares flight test and prediction pitch angles. In both cases, pitch angles increase 
with airspeed, which is expected, as the drag of the vehicle increases with airspeed by a factor of the squared velocity. The parabolic trend of the prediction pitch results is contributed to the squared velocity. However, a linear trend is present in the flight test data. This is due to the SkyRanger control model established by Aeryon Labs. Figure $4.9 \mathrm{~b}$ shows the difference between flight test and prediction pitch results at six selected airspeeds. Overall, the pitch comparisons are within $+/-3^{\circ}$ between the airspeed range observed.

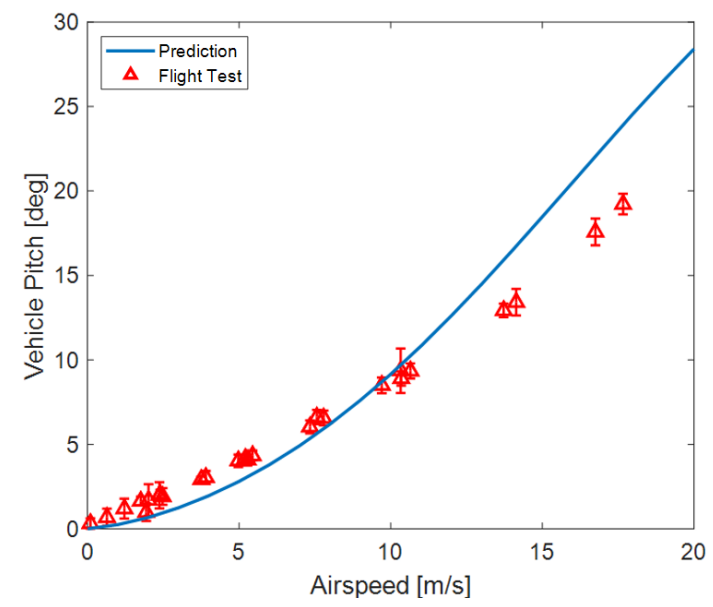

(a) Vehicle pitch.

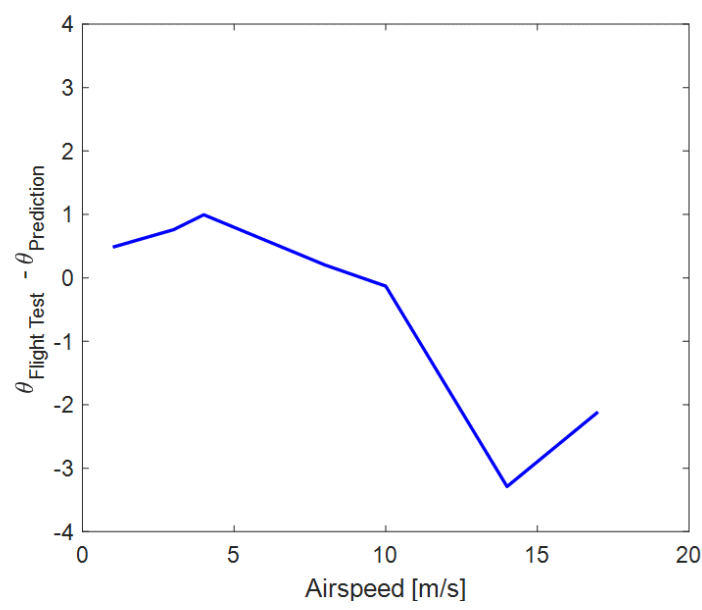

(b) $\theta_{F T}-\theta_{P}$.

Figure 4.9: Vehicle pitch comparison between flight test and prediction data of a SkyRanger vehicle.

Demonstrating agreement in rotor speed trends between lead and rear rotors shows an improvement towards developing reliable prediction models when compared to the original multirotor vehicle performance model from Ref. [14. The improvements made in the prediction method to determine the performance characteristics of a multirotor vehicle is essential toward the improvement of control laws that set the rotor speeds for a given flight mission. For instance, it could be beneficial for the control systems model of the SkyRanger that controls the pitch of the vehicle to reflect the quadratic model provided by the performance prediction method. Overall, the performance prediction results agree well with the flight test data presented, especially at airspeeds less than $10 \mathrm{~m} / \mathrm{s}$. 


\section{CHAPTER 5}

\section{ORIENTATION STUDY}

Changing flight orientations throughout a flight mission can have an effect on the power needs of the multirotor vehicle and subsequently affect its endurance. For vehicles with camera payloads, the ideal flight orientation is often based on an orientation where the rotors and landing gear are not in the frame of the camera; However, for applications without a critical orientation, for example, because either the camera is off or the payload type is, related to package delivery, orientating the rotor positions in a specific direction relative to the freestream flow can have flight performance benefits and lead to longer flight times.

This section compares predicted vehicle performance of a multirotor vehicle in steady level forward flight in diamond and square configurations. The performance analysis includes comparing rotor speeds, rotor thrust, rotor and vehicle power, as well as residual rolling, pitching, and yawing moments of vehicle components during steady level flight. In Ref. 14, the differences in vehicle power between were compared diamond and square configurations, but trim and body interference models were not considered for this analysis. The predicted influence of the rotor-interference and fuselage-interference velocities on vehicle performance is further explored.

The assessed vehicle was based on an Aeryon SkyRanger with a camera payload, as shown in Fig. 3.3 and using four T-Motor 18x6.1 rotors. The T-Motor, which is not the standard rotor configuration used for the Aeryon SkyRanger, was selected because it was used as part of previous research in Refs. 17 and 14. It must be noted that an 18-inch propeller is too large to be installed on the SkyRanger vehicle, resulting in a physical interference between rotor and body and overlapping rotor planes. In order to 
accommodate the larger rotor size, the SkyRanger arm lengths of the prediction model were increased from $0.20 \mathrm{~m}$ to $0.28 \mathrm{~m}$ to satisfy the same arm length to rotor radius ratio as the proprietary 15 -inch propeller that is typically used on the SkyRanger.

Extending the arm length increased the parasitic drag results of the modified SkyRanger compared to using the original vehicle geometry. For example at $5 \mathrm{~m} / \mathrm{s}$, when the arm length was increased by $40 \%$, the predicted parasitic drag of one arm also increased by $40 \%$. The total parasitic drag of the SkyRanger vehicle only increased by $9 \%$. The arm component weight was not modified.

\subsection{Vehicle Performance}

In this section, thrust, rotor speed, and power requirements for the modified SkyRanger vehicle are compared between diamond and square configurations, as shown in Fig. 2.1, when flying in steady level flight. The vehicle performance is predicted using the algorithm outlined in Fig. 3.10 This means, the aircraft was trimmed in pitch while considering the interference of the rotors on each other as well as the influence of the flowfield that is disturbed by the fuselage.

Figure 5.1 shows the rotor thrust, rotor speed, and rotor power results for the modified SkyRanger while flying in diamond and square configurations over a range of airspeeds. Rotor thrust values are predicted using the force and moment equilibrium modules. Rotor speeds and rotor power are predicted using the rotor table lookup routine using thrust required for force and moment trim, inflow velocities, and inflow angles as function inputs. Rotor inflow velocities and inflow angles are predicted using the rotor interaction model. 


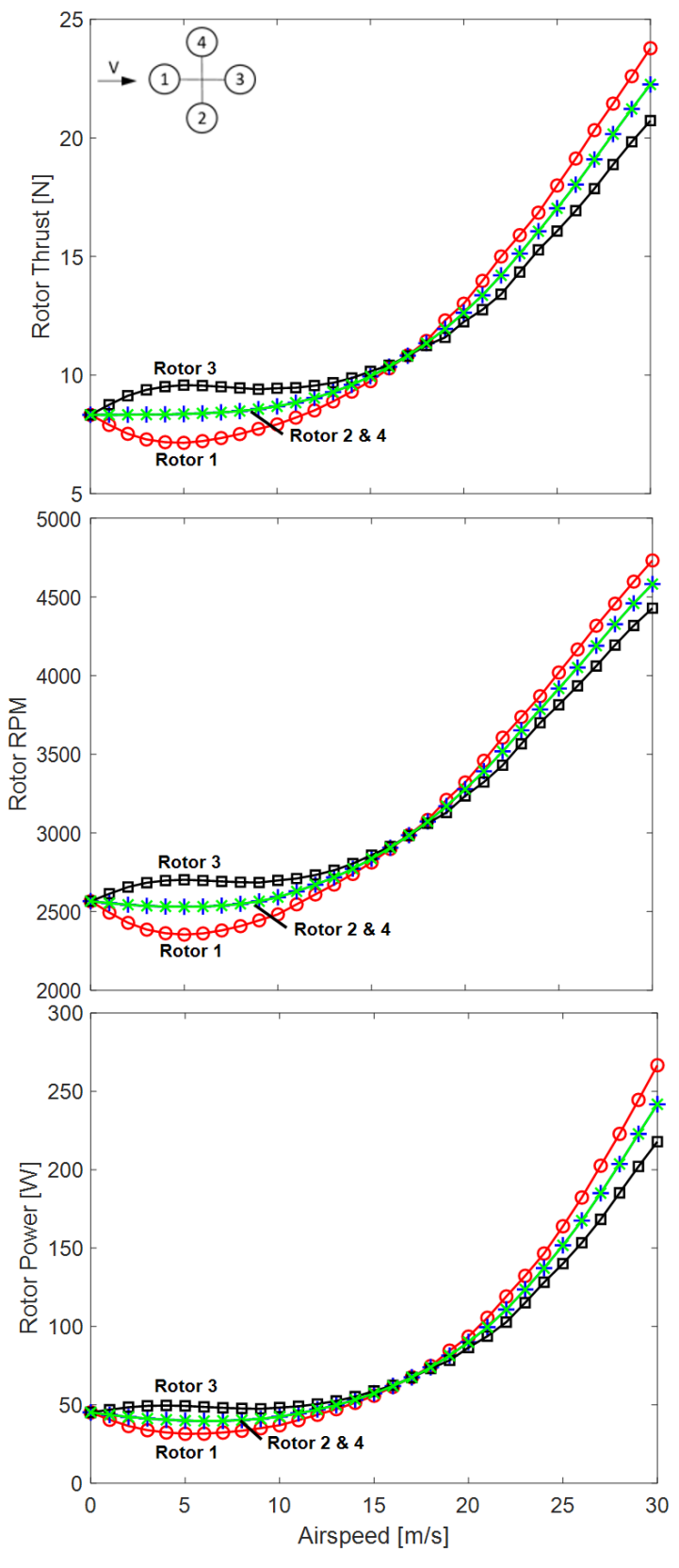

(a) Diamond configuration.

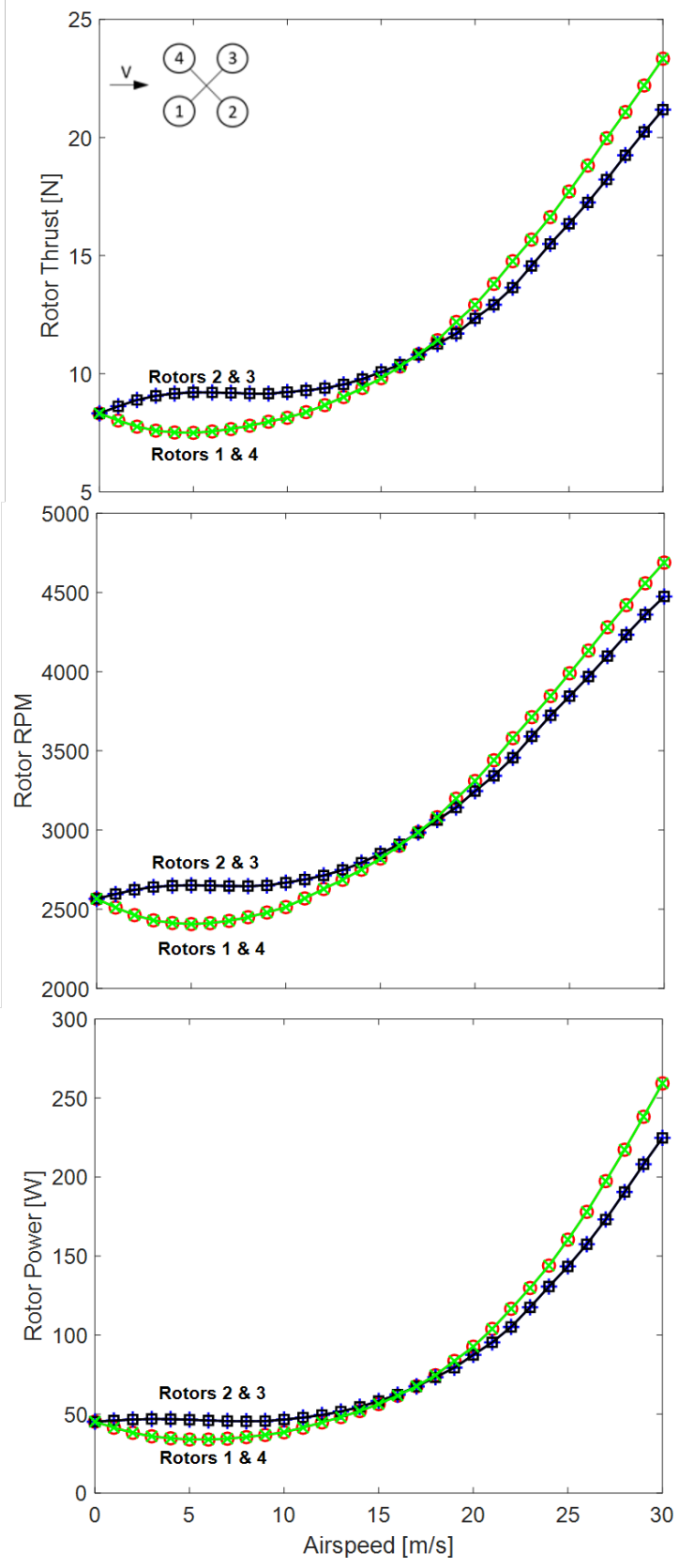

(b) Square configuration.

Figure 5.1: Rotor thrust, speed, and power required for straight and steady longitudinal flight over a range of flight speeds in diamond and square configurations. 
At all airspeeds, the force trim of the vehicle is maintained while the thrust of the lead and rear rotors are adjusted to achieve pitching moment trim. At hover, the symmetry of the arms, motors, landing gear, payload and fuselage about the vehicle's vertical axis of the vehicle results in all rotors having equal thrusts to support the weight of the vehicle. There is no moment trim correction at hover for the modified SkyRanger. With increasing airspeed, the differential thrusts of the the lead and rear rotors is needed in order to achieve longitudinal trim. The two side rotors in the diamond configuration have equal thrust due to geometric symmetries about the longitudinal plane of the vehicle. Similarly, both lead rotors and both rear rotors in square configuration have equal rotor thrust. At lower airspeeds between 1-5 m/s, the increase in rear rotor thrust and decrease in lead rotor thrust in both configurations are due to resolving the total vehicle pitching moment to zero. A pitching moment decomposition of vehicle force components is outlined later in this chapter. The upper and lower thrust peaks around 5 $\mathrm{m} / \mathrm{s}$ of the rear and lead rotors, respectively, correspond to the peak sum of rotor pitching moments. In addition, as airspeed increases past $10 \mathrm{~m} / \mathrm{s}$, the total thrust requirements of the vehicle increase to meet the propulsion requirements due to the increase in parasitic drag. The increase in parasitic drag results in greater negative pitching moment contributions resulting in the crossover of thrust requirements after $15 \mathrm{~m} / \mathrm{s}$.

Results for rotor speed and rotor power are interpolated within the rotor performance lookup tables based on rotor thrust, inflow velocity, and inflow angle. The rotor speed and rotor power of the lead and rear rotors in Fig. 5.1 are primarily driven by the thrust requirements that are determined by the moment trim module. However, rotor interference and fuselage interference velocities influence the inflow condition at each rotor which impacts the rotor speed and power results that are required to maintain a desired thrust.

Removing moment trim from the prediction method algorithm is useful for seeing the impact of the total interference velocity on the rotor performance when all four rotors are assigned the same rotor thrust. Figure 5.2 shows rotor speed results when only force trim is applied and no differential thrust for moment trim. Subsequently, the difference between rotor speeds is solely due to the rotor and fuselage interferences that change the inflow conditions of the rotor. Notably in forward flight, the rear rotors experience increasing downwash and increasing inflow angles due to the mutual interactions between the rotors. Although not shown in Fig. 5.2, at lower airspeeds the lead rotors experience upwash effects from the rear rotors and, in fact, have negative inflow angles. The side rotors see mostly the upwash 
contributions of the lead, rear and opposing side rotor. Inflow velocities and inflow angle results for this performance analysis are discussed later in this chapter. Ultimately the downwash and upwash effects applied to the rotors change the inflow velocity and inflow angle of each rotor. The changes in inflow conditions change the rotor speed and rotor power results retrieved through the performance lookup tables in the prediction method. 


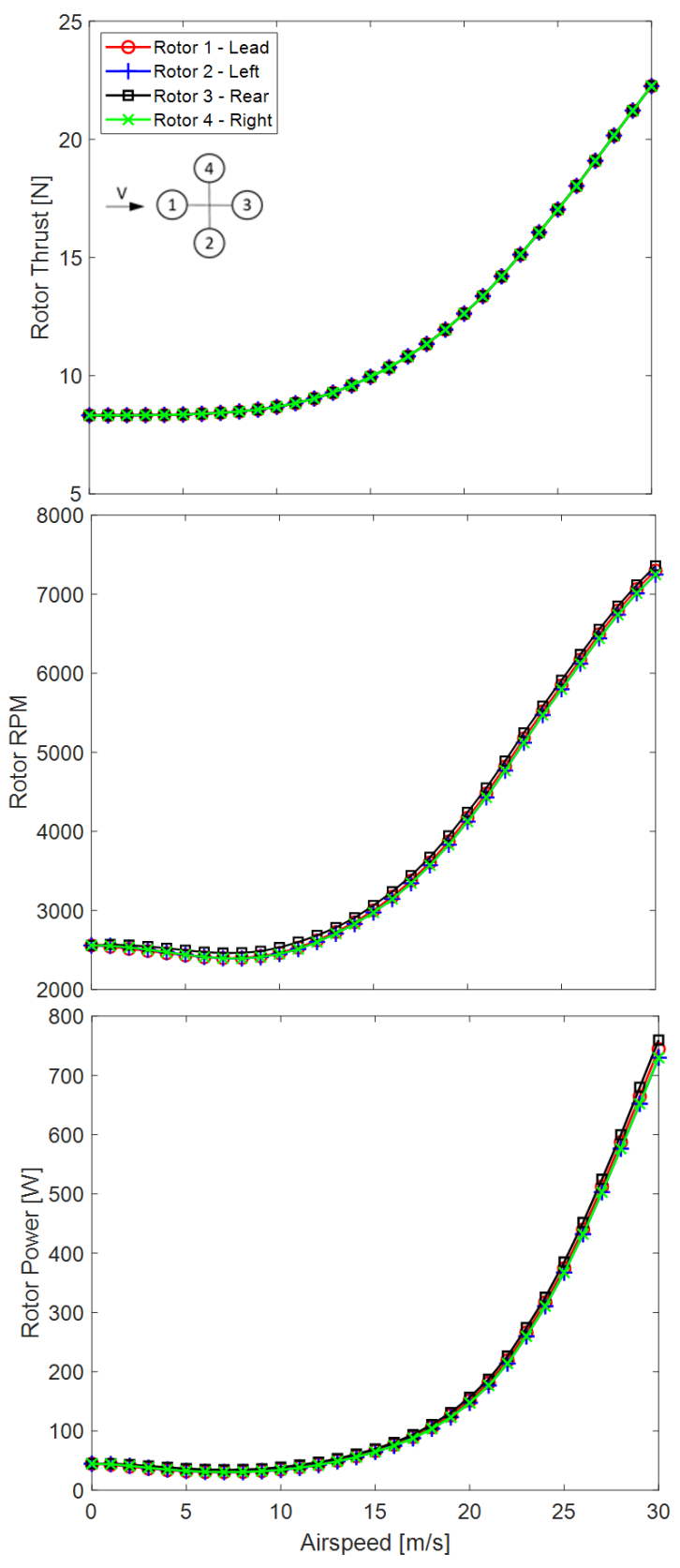

(a) Diamond configuration

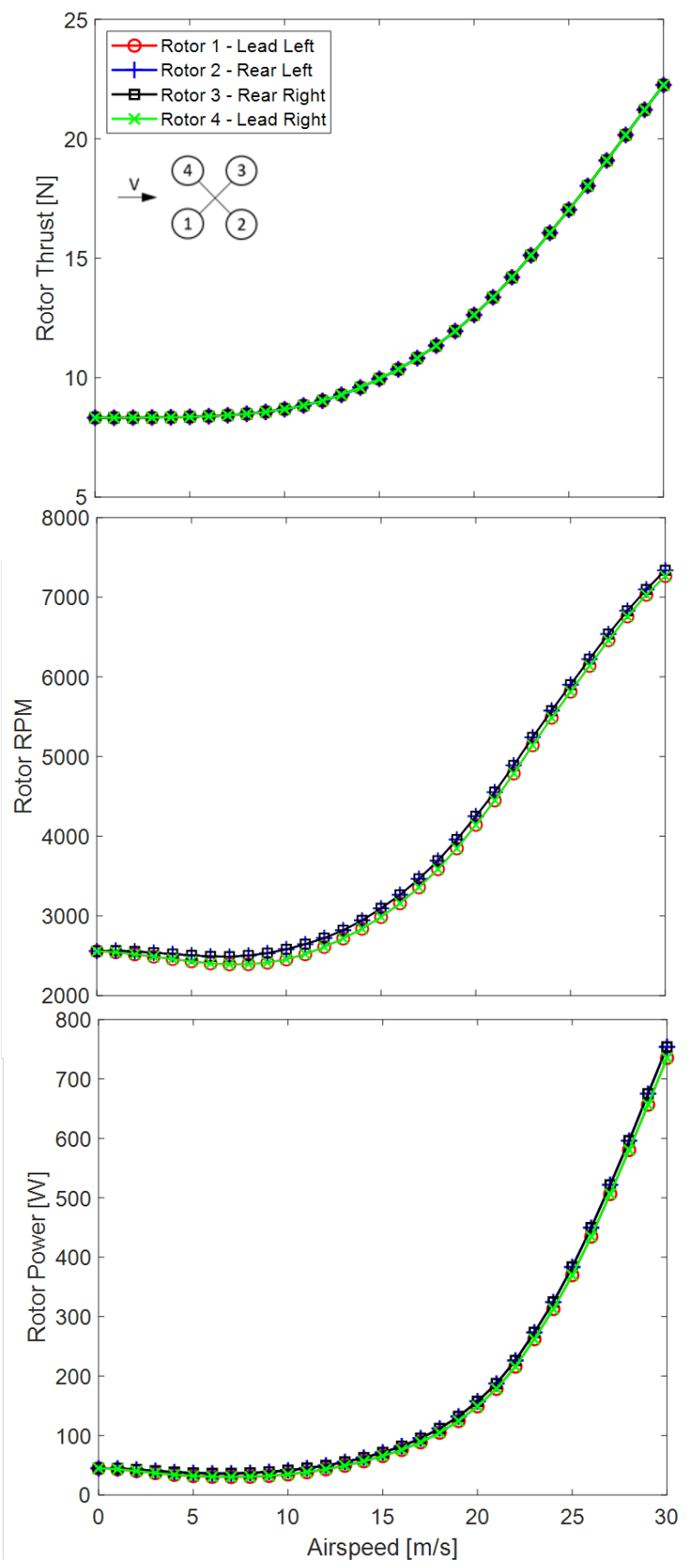

(b) Square configuration.

Figure 5.2: Rotor thrust, speed, and power required for straight and steady longitudinal flight over a range of flight speeds in diamond and square configurations. 
Figure 5.3 shows the total vehicle power require of the diamond and square formations with and without the moment trim. Total vehicle power is the sum of the rotor powers and the parasitic power of the fuselage. At hover, all configurations will have the same power requirements. When all four rotors are assigned equal thrust, that is without trimming the aircraft, the difference in vehicle power between diamond and square configurations is up to $5 \%$ with the square configuration requiring more power. The power advantage in diamond configuration is due to only one rear rotor experiencing the downwash effects of the lead rotor rather than two rotors experiencing downwash effects in square configuration. Also, the diamond configuration has a larger overall span, thus, analogous to fixed wings, has the lesser span loading, which means less induced drag and power. The higher the downwash the greater the rotor speed and power required to maintain thrust. These results are the same as the results discussed in Ref. 14. After applying moment trim, the power benefits of flying in diamond configuration diminish as the vehicle power between the two configurations is only $0.005 \%$, the higher power required for diamond configuration. The higher power in diamond configuration, as seen in Fig. [5.1, is due to the higher power requirements of the rear rotor and side rotors compared to the two rear rotor power requirements in square configuration.

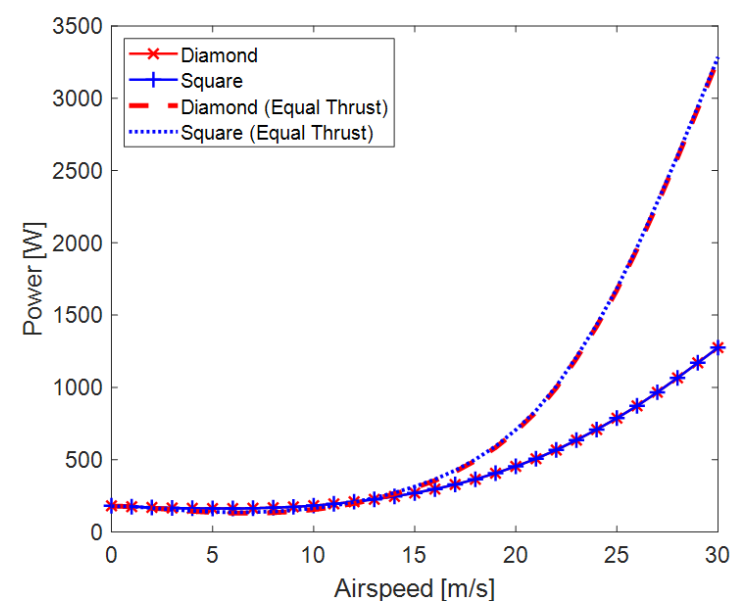

Figure 5.3: Total vehicle power in diamond and square configurations. 


\subsection{Interference Velocities}

The total interference velocities are studied in this section in order to investigate the impact of rotor and fuselage interference velocities on the inflow velocity and inflow angle of each rotor in both square and diamond configurations of a quadrotor.

\subsubsection{Rotor Interference Velocity}

Figure 5.4 shows the predicted rotor interference velocities present at the hub of each rotor due to the flowfields of the surrounding rotors of the diamond and square configurations. The total rotor interference velocities are predicted using the sum of velocities that the other rotors induce at the rotor of interest, as discussed in Section 3.3. Positive values indicate upwash and negative velocities indicate downwash. Figure 5.5 shows the components that contribute to the rotor interference velocities that are presented in Figure 5.4 .

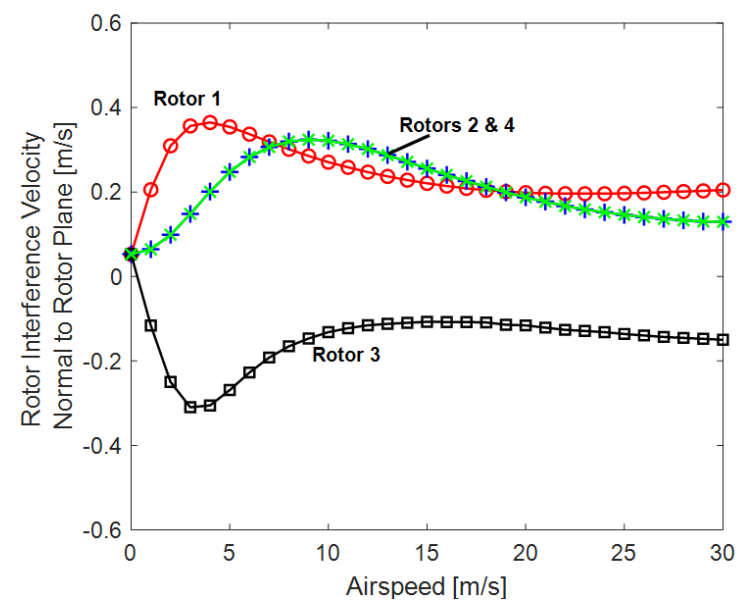

(a) Diamond

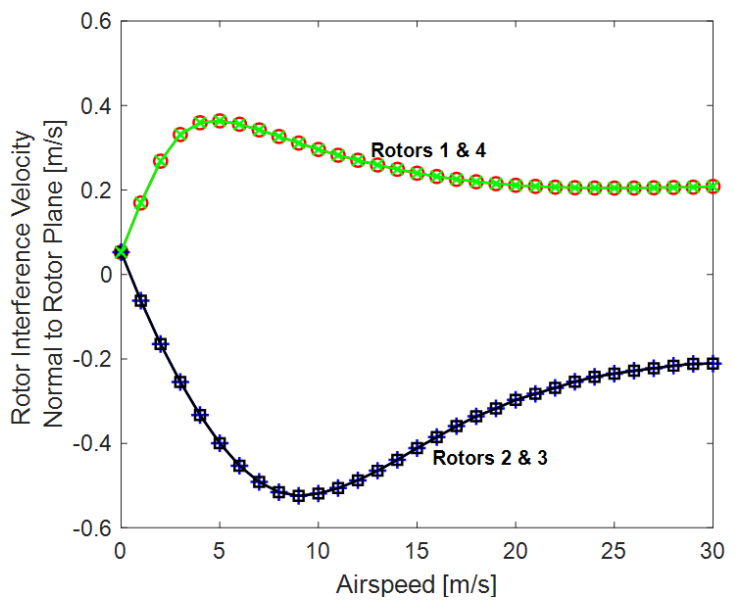

(b) Square

Figure 5.4: Rotor interference velocities applied normal to the rotor plane of each rotor in diamond and square configurations. 

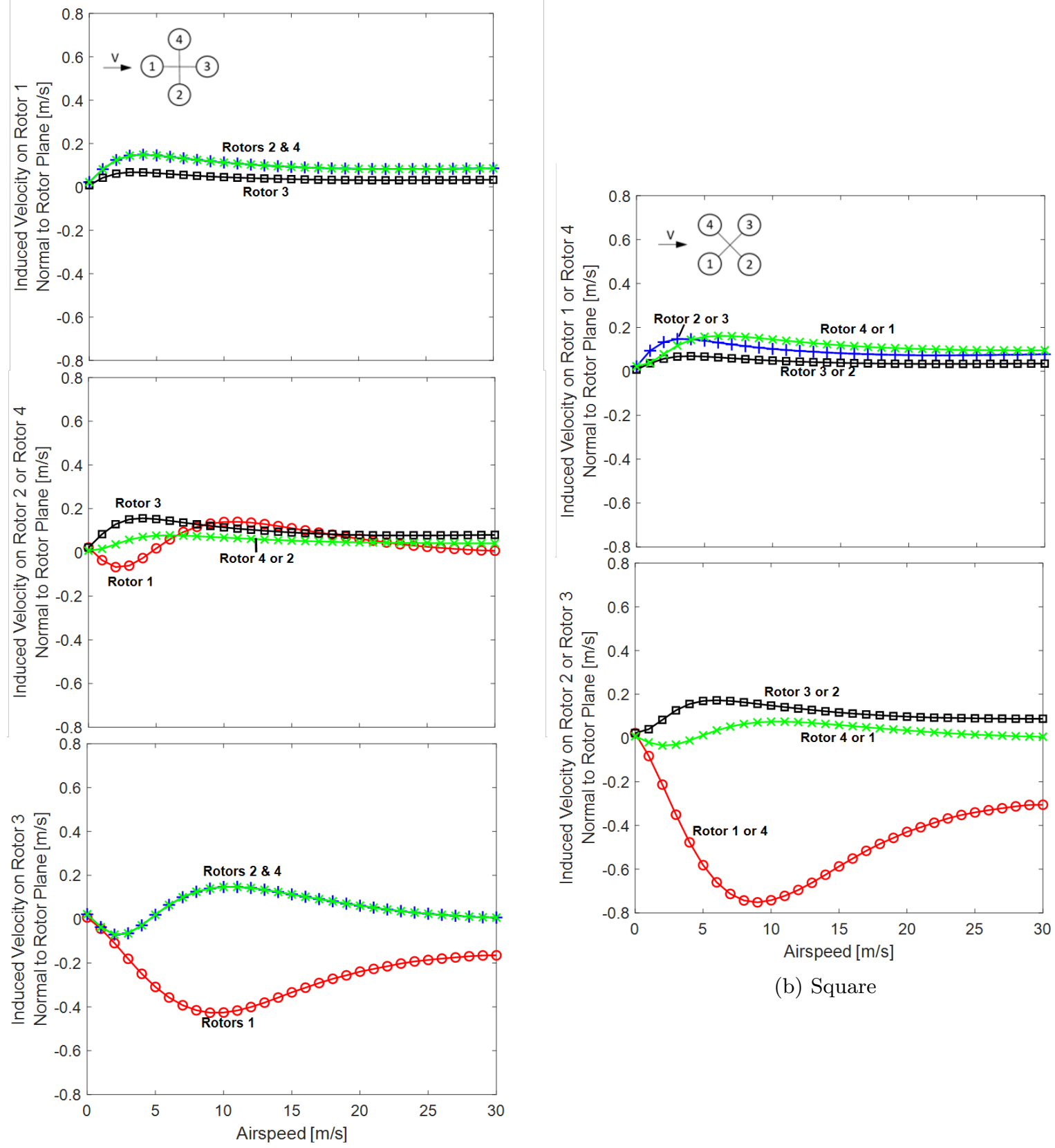

(b) Square

(a) Diamond

Figure 5.5: Induced velocities at each rotor of a vehicle in diamond and square configuration. 
The rotor interference velocities for at each airspeed point are functions of the airspeed, vehicle pitch, air density, thrust, rotor speeds, rotor diameter, and distance between rotors. At hover, all rotors experience the same amount of upwash due to the other rotors. During forward flight, the rear rotors in both configurations immediately enter the downwash of the lead rotors and the lead rotors experience an upwash from the rear and side rotors. Once the multirotor vehicle is in forward flight, the rear rotors experience increasing downwash and the lead rotors upwash as the airspeed increases. In the case of the two side rotors of the diamond configuration, the rotors see upwash benefits from the lead rotor and of each other. Furthermore, with increasing flight speed, the lead rotor of the diamond configuration varies slightly and seems to diminish quickly. In the square configuration, a growing increase in negative downwash interference velocity is observed on the rear rotors as they are in the full wakes of the lead rotors. Between diamond and square configurations, Fig. 5.4 shows that the rear rotors in the square configuration see a greater downwash than the single rear rotor of the vehicle in diamond configuration. This is attributed to the positioning of the rotors. In diamond configuration, the lead and rear rotors are in diagonally opposing positions in the quadrotor configuration. In square configuration, the lead and rear rotors are side opposing rotors so the distance between rotors is smaller. The smaller distance between lead and rear rotors contributes to the greater downwash applied to the rear rotor.

Rotors in the direct downwash of the lead rotors have to "work" harder. As the rotor interference velocity vectors are added to the freestream velocity, the inflow velocity and inflow angles applied to the rear rotors require faster rotor speeds and higher power requirements for given conditions. For example, Fig. 5.2 demonstrates the influence of rotor interference velocities on the rotor speed results when all rotors have an equal thrust requirement. The predicted rotor speeds of the rear rotors are greater than the lead rotors of the same thrust requirement. There are also performance benefits due to wake effects when the lead rotors are in the direct upwash of the rear rotors. At hover, all rotors see the same upwash benefit and a rotor within a multirotor configuration will require a lower rotor speed and power requirement at hover than a single rotor. When upwash is applied to a rotor, the inflow velocity and inflow angle decrease resulting in lower rotor speeds and power required to maintain the required thrust of the rotor.

In order to see why the interference velocity peaks at $4 \mathrm{~m} / \mathrm{s}$ for the lead rotor for the diamond configuration case, trends in rotor thrust and rotor speeds must also be observed. Consider the peak predicted rotor interference velocity applied to side rotors 2 and 4 in Fig. 5.4a. The predicted peak 
interference velocity occurs around $8 \mathrm{~m} / \mathrm{s}$, which is the same airspeed where the minimum rotor speed occurs, as shown in Fig. 5.1. The peaks in rotor interference velocities closely resemble the peaks observed in the rotor thrust and rotor speed trends in Fig. 5.1. Ultimately, the interference velocities are a result of the combination of constantly changing rotor thrusts, vehicle pitch angles, and advance ratios.

At low airspeeds, the interference velocities are a significant percentage of the freestream velocity. For example, consider the lead rotor in diamond configuration. At $1 \mathrm{~m} / \mathrm{s}$ airspeed the rotor interference velocity applied to rotor 1 is almost $0.3 \mathrm{~m} / \mathrm{s}$, close to $30 \%$ of the total relative airspeed applied the rotor. At airspeeds greater than $4 \mathrm{~m} / \mathrm{s}$, the rotor interference velocity is $10 \%$ of the total relative airspeed. For speeds faster than $4 \mathrm{~m} / \mathrm{s}$, the rotor interference velocities gradually decreases as contributions of the horizontal freestream velocity grows much faster than the rotor interference velocity.

Incorporating the rotor interference module into the multirotor vehicle performance model is essential to capturing the upwash and downwash effects of rotor interactions on the vehicle performance, particularly at lower airspeeds when the rotor interference velocity is a significant percentage of the total flow through the rotor. At higher velocities, particularly over $10 \mathrm{~m} / \mathrm{s}$, skew angles remain relatively constant, the freestream velocity dominates vehicle performance as form drag increases quadratically, and the rotor interference velocities play a less dominate roll in predicting rotor speed and power.

\subsubsection{Fuselage Interference}

The fuselage interference velocities at the hub of each rotor that are caused by the flow about the central body of the fuselage are shown in Fig. 5.6. The fuselage interference velocities are dependent on the vehicle airspeed, the pitch angle, and the distance between the rotor hubs and the surface of the central body that is assumed to have a spherical shape. The fuselage interference velocities are estimated using a potential flow model, for which the fuselage and its flow disturbance are modelled using a three dimensional doublet in a uniform flow. Fuselage interference velocities are shown in terms of the tangential and normal velocity components. As shown in Fig. 2.8, the tangential components are along the rotor disk and normal components are through the rotor disk. See Fig. 3.4, which illustrates the body interference velocity vector added to the freestream velocity relative to the rotor plane reference frame. 


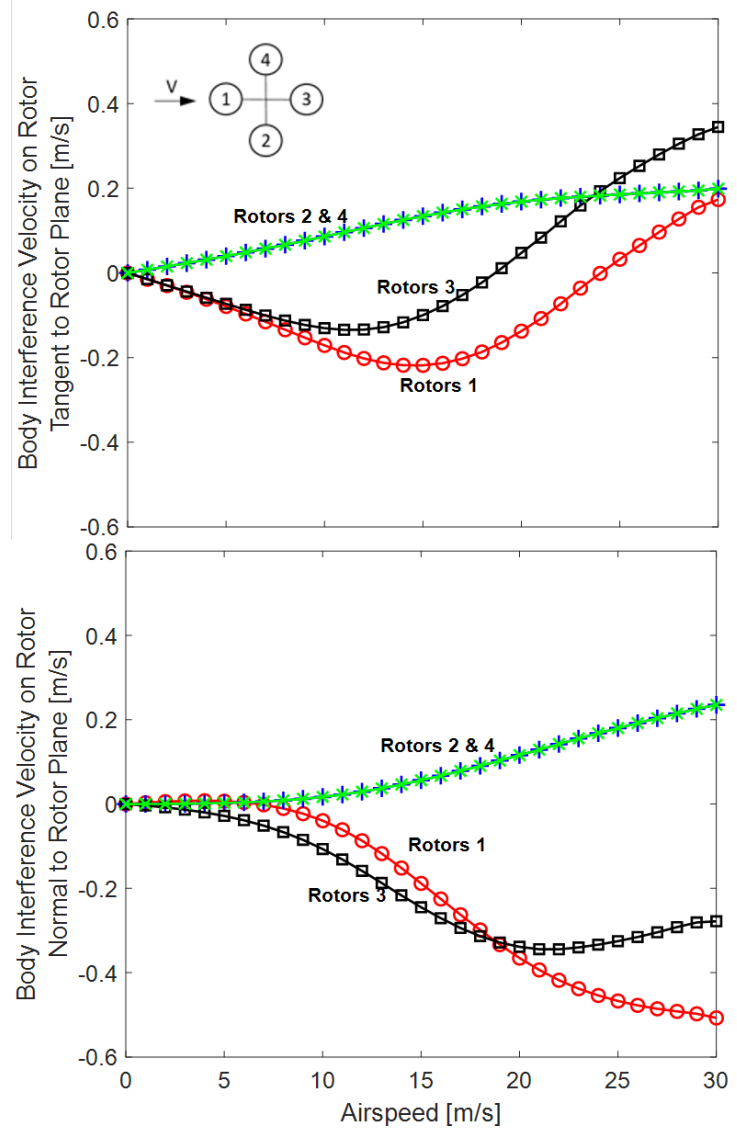

(a) Diamond configuration

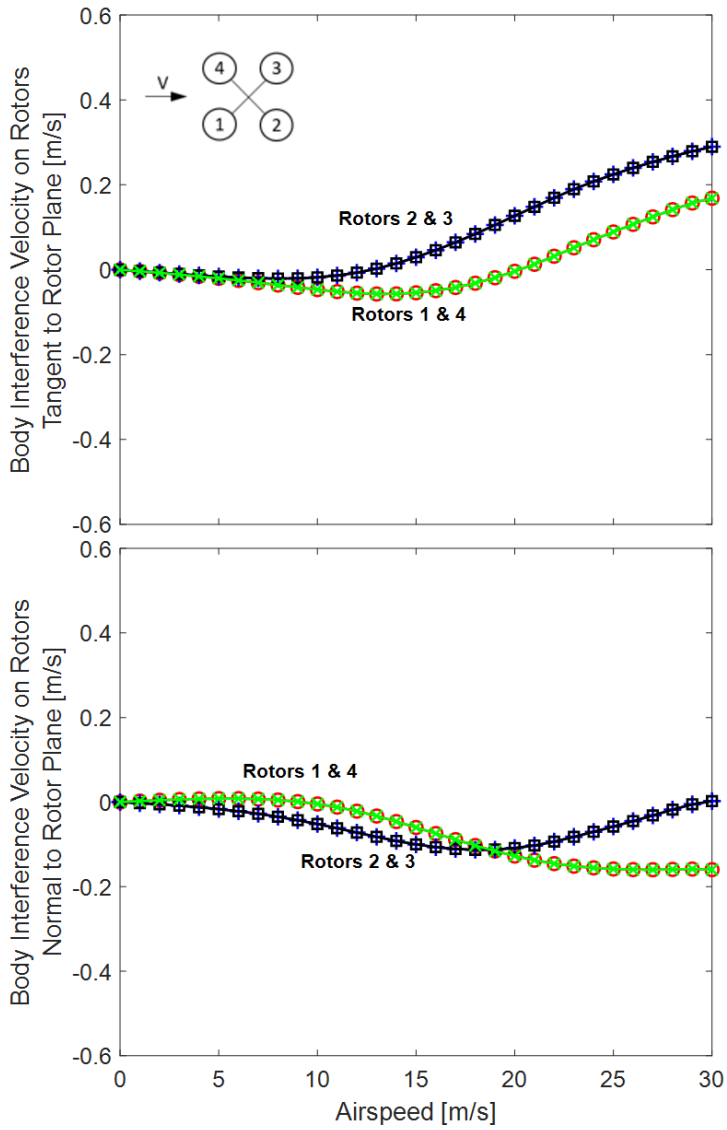

(b) Square configuration

Figure 5.6: Fuselage interference velocities at rotor hubs of the diamond and square configurations. Velocities are in rotor reference frame.

The magnitudes of the predicted fuselage interference velocities shown in Fig. 5.6 are comparable to the magnitudes of the rotor interference velocities shown in Fig. 5.4. Compared to the freestream velocity, the fuselage interference velocities are minimal, less than $2 \%$ of the freestream velocity after $5 \mathrm{~m} / \mathrm{s}$. However, note that similar to the rotor interference, the interference velocity of the fuselage is evaluated at the rotor hub location only, and not applied incrementally over the blade sections of the rotor.

Fig. 5.7 shows an example of the fuselage interference velocity on a point of interest with changing distance, $r$, away from the centre of sphere with a radius, $R$, at a constant freestream velocity. The changing distance, $r$, represents the different rotor disk positions from the surface of the central body. 
Three freestream velocities $5,10,15 \mathrm{~m} / \mathrm{s}$, are compared at a position $90^{\circ}$ from the horizontal freestream vector. A $r / R$ ratio of 1 indicates the point of interest is located on the rotor surface of the body. With increasing distance between the surface of the central body the velocity decreases rapidly.

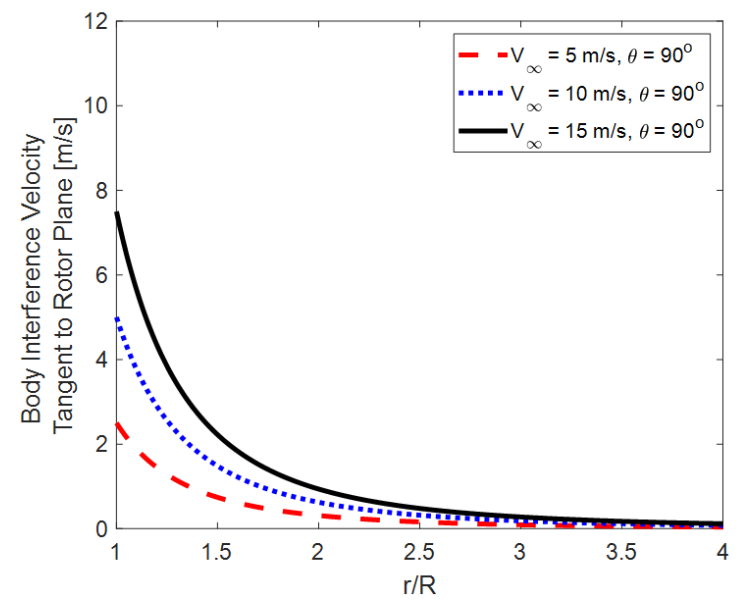

(a) $V_{x}$ at $\theta=90^{\circ}$

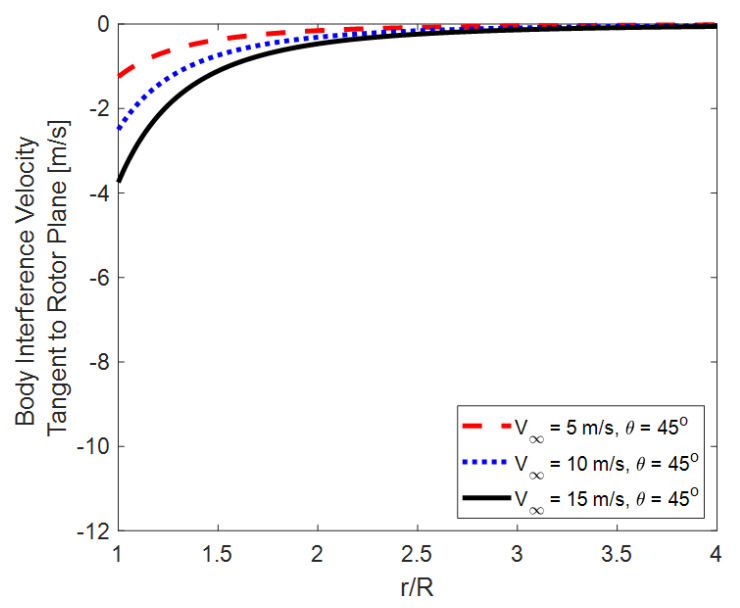

(c) $V_{x}$ at $\theta=45^{\circ}$

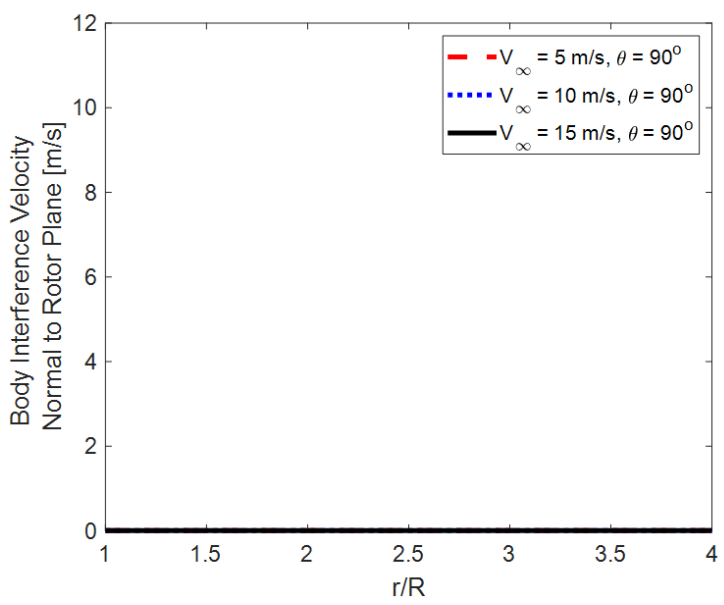

(b) $V_{z}$ at $\theta=90^{\circ}$

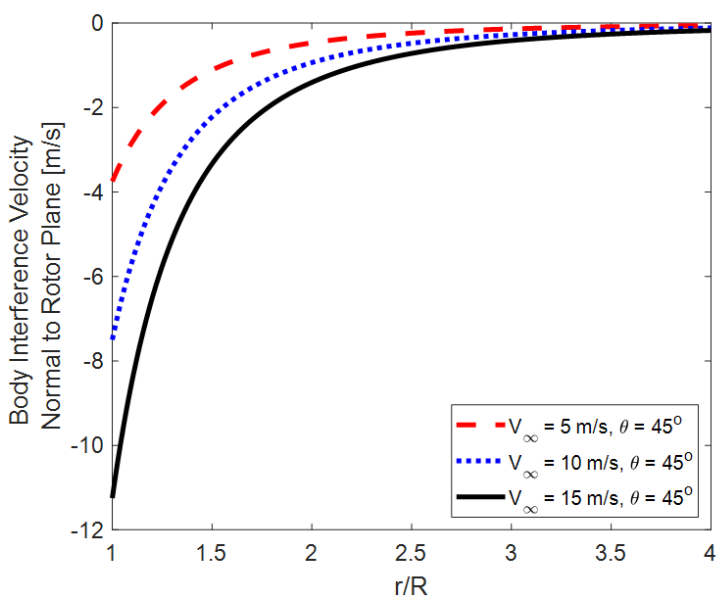

(d) $V_{z}$ at $\theta=45^{\circ}$

Figure 5.7: Body interference velocity applied to point of interest at distance, $r$, and angle, $\theta$, about a sphere with radius, $R$. Velocities in horizontal reference frame.

For all the velocity cases, the body interference on the surface of the body is exactly half of the freestream velocity. This is expected as the tangential velocity component on the surface of the sphere is $q_{\theta}=-\frac{3}{2} U_{\infty} \sin \theta$ and subtracting the radial velocity component at $90^{\circ}$ from the freestream velocity gives a result of half of the freestream velocity. In the case of the modified SkyRanger vehicle design 
used in this study, the rotors are located around four times the distance compared to the body radius. As shown in Fig. 5.7, body interference velocities at a distance four times the radius of the central body are less than $0.01 V_{\infty}$ of all freestream cases. If the rotor radii were smaller and the rotor rubs closer to the central body, the fuselage interference results would show a greater impact on the overall inflow velocity of each rotor. While the impact of body interference velocity on each rotor of the modified SkyRanger vehicle is low, using a fuselage interference model can be useful in predicting the influence of the fuselage for other multirotor designs of various central body shapes or rotor sizes.

\subsubsection{Inflow Velocity and Inflow Angle}

In this section, the total velocity contributions and resultant inflow angles of each rotor are discussed. The inflow velocities and angles are required parameters used in the rotor performance lookup tables that are used to determine the rotor speeds of the vehicle. The velocity and angle conventions used in this analysis are in accordance to Figure 3.4

Figures 5.8 and 5.9 show the composition of inflow velocities of the diamond and square configurations, respectively. Each subfigure shows the fuselage interference, rotor interference, and freestream contributions towards the total inflow velocity. The velocity components that are tangential to the rotor disk are plotted in Figs. 5.8a and 5.9a. The velocity components that are normal to the rotor disk are plotted Figs. $5.8 \mathrm{~b}$ and $5.9 \mathrm{~b}$ Lateral velocities were not studied here.

The rotor interference velocities due to the other rotors only produces velocities that are normal to the rotor disk, since all four rotors are in the same plane. The body interference velocities also have little contributions, less than $0.2 \mathrm{~m} / \mathrm{s}$ for all vehicle speeds, to the overall inflow velocity parallel to the rotor plane. As discussed in the fuselage interference section of this chapter, for this vehicle case and 18-inch diameter rotors the distance between the rotor hubs and the body surface is sufficiently large for the body interference to have little influence on the total resultant inflow velocities. 

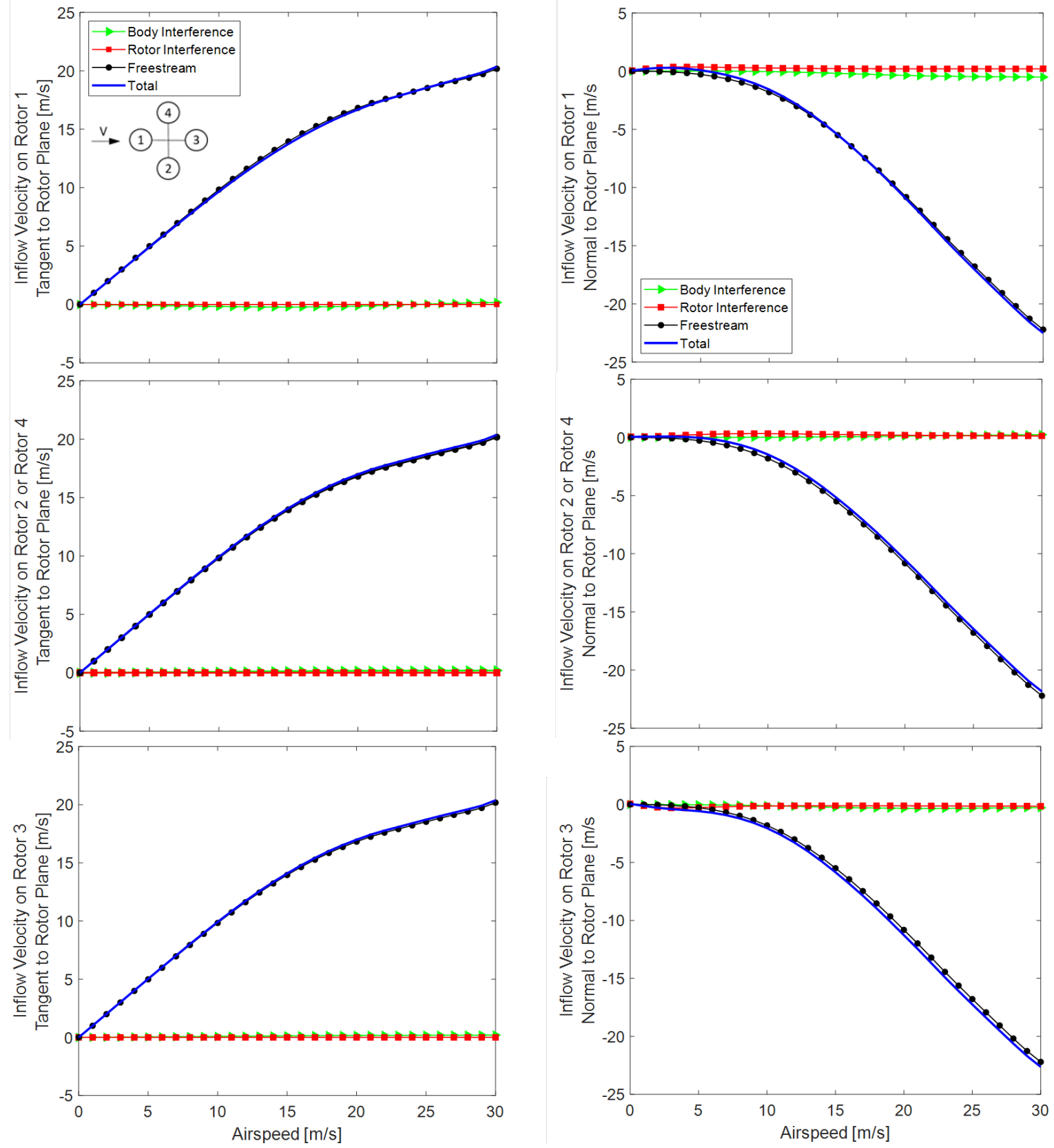

(a) Tangent Inflow

(b) Normal Inflow

Figure 5.8: Inflow velocity components tangent and normal to rotor plane. Vehicle in diamond configuration. 


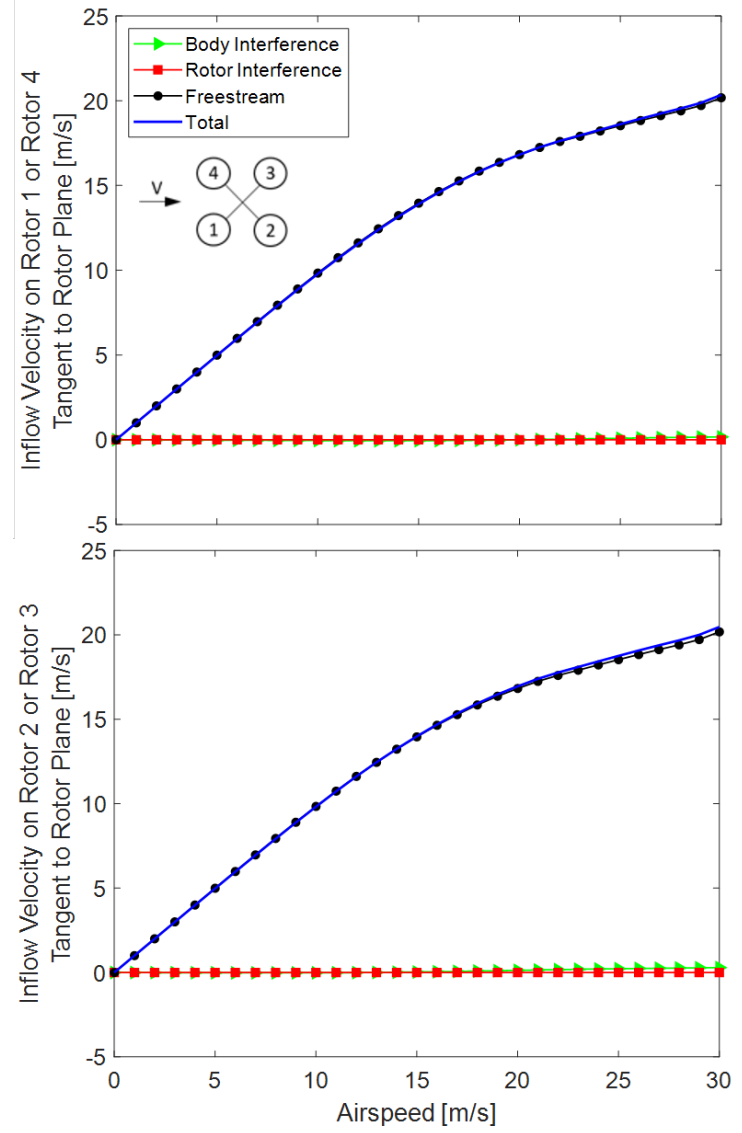

(a) Tangent Inflow

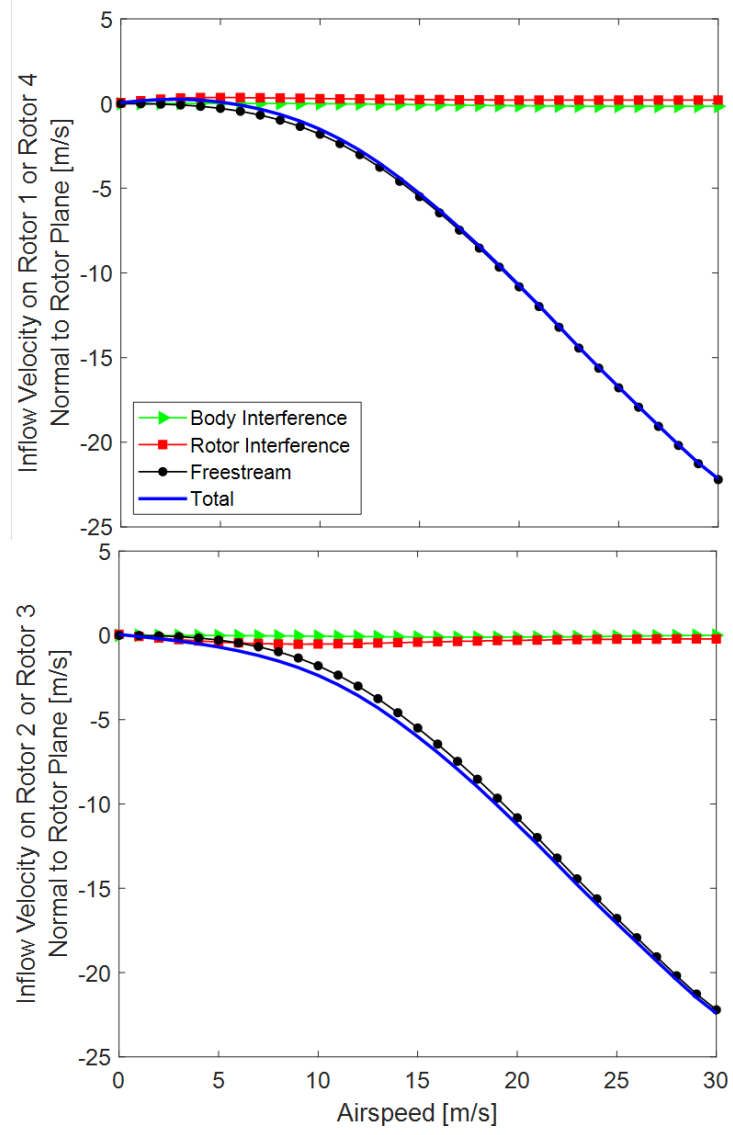

(b) Normal Inflow

Figure 5.9: Inflow velocity components tangent and normal to rotor plane. Vehicle in square configuration.

The results of the normal components of the total inflow reflect the addition of the interference velocity vectors to the freestream vector, as noted in the difference between the total inflow velocities and freestream velocities in Figs. $5.8 \mathrm{~b}$ and $5.9 \mathrm{~b}$. Generally, the interference velocities have the greatest impact on modifying the freestream flow at airspeeds below approximately $10-15 \mathrm{~m} / \mathrm{s}$. Total inflow velocities of lower magnitude than the freesteam, such as the lead and side rotors in diamond configuration or the lead rotors in square configuration, indicate that the total interference applies an upwash to the rotors reducing the normal component of inflow velocity.

Where the overall changes between the magnitude of freestream and resultant inflow velocities are seen to be minimal in the SkyRanger configuration, the influence of the interference velocity vectors on 
the effective inflow angle is more prominent as indicated in Fig. 5.10 which shows the resultant inflow angle of each rotor. The inflow angles for each rotor at hover are not shown, but are $-90^{\circ}$, as an upwash. Figure 5.10 also shows the difference in angles between the inflow angle and the pitch attitude required for longitudinal flight over a range of airspeeds. This differential angle is a measure of the angle at which the rotor wakes depart from the pitch angle. Without the implementation of the rotor and body interference models, the inflow angles and the pitch angles are equal.

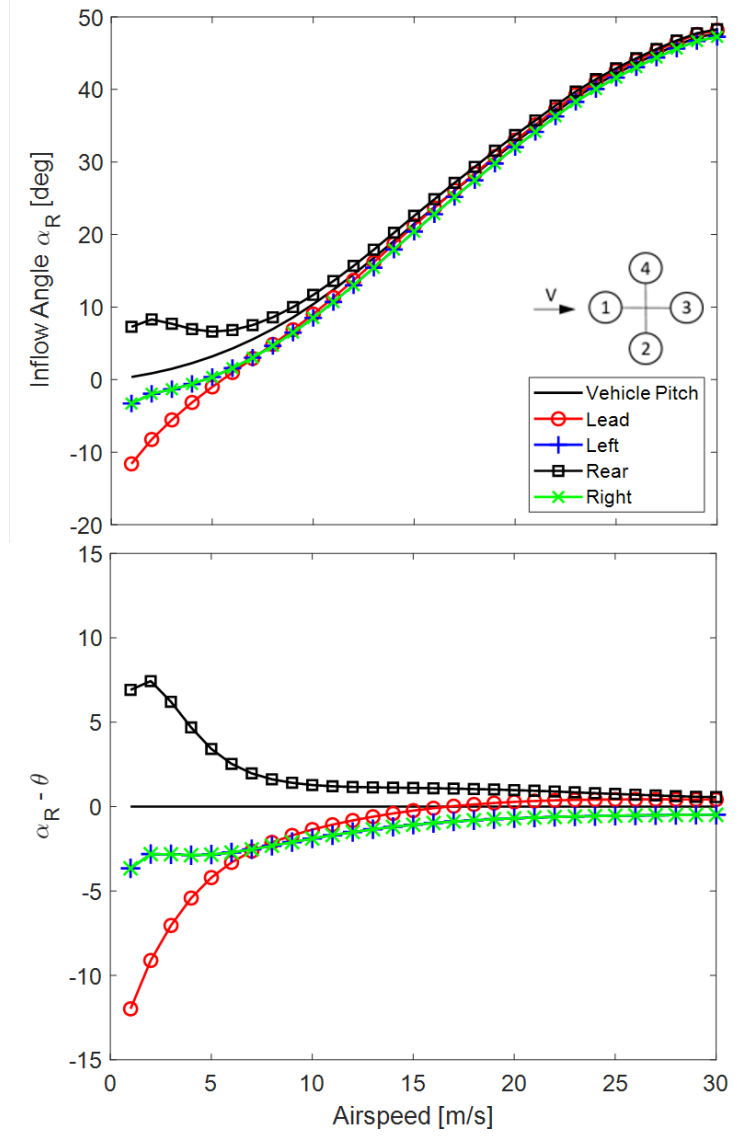

(a) Diamond

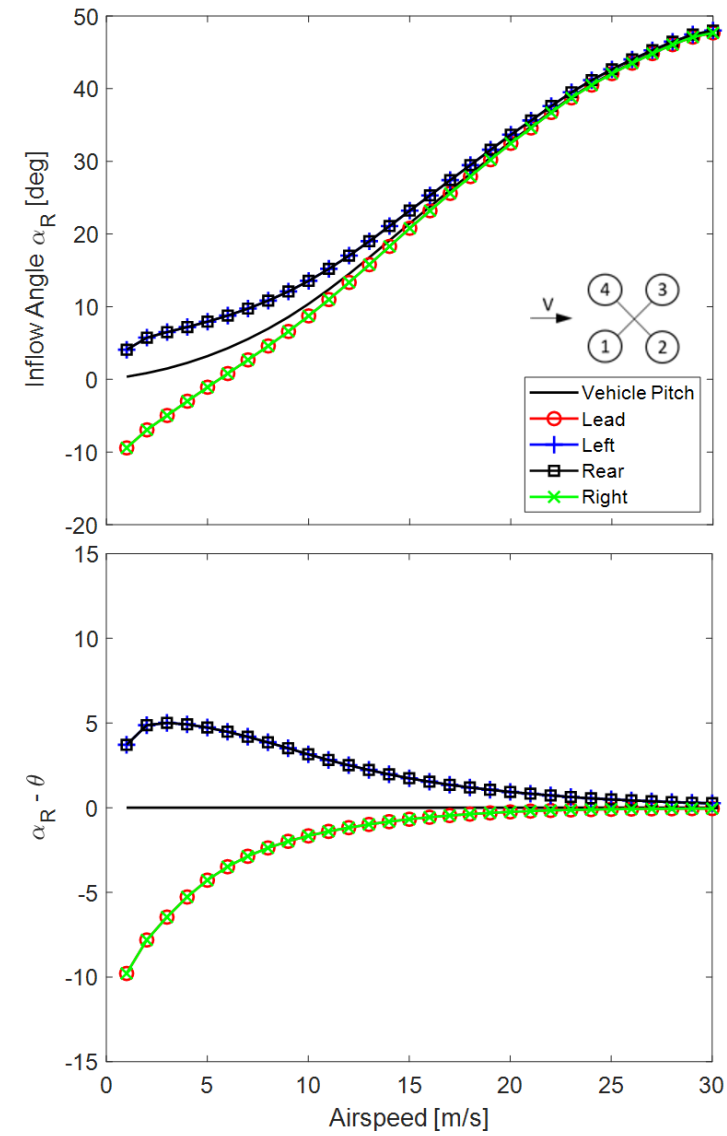

(b) Square

Figure 5.10: Change in inflow angle due to flow interference effects compared to vehicle pitch angle.

The interference velocities changes the effective inflow angle of each rotor. For example, the rear rotors in diamond and square configurations see a greater resultant inflow angle of $5^{\circ}-8^{\circ}$ greater than the pitch angle under $4 \mathrm{~m} / \mathrm{s}$ due to the downwash contributions of the rotor and body interference 
velocities. Inversely, the leading rotors see a reduction in inflow angle of $-10^{\circ}-12^{\circ}$ from the pitch angle due to the upwash contributions of the trailing rotors. The side rotors see the upwash contributions of the lead and trailing rotors, as well as upwash benefits from each other. At higher airspeeds, the inflow angles and inflow velocities of each rotor converge onto the results of freestream and pitch angle, respectively.

The impact of the interference velocities on the rotor performance is seen in the performance results in Figs. 5.2, where the thrust requirement is the same for all rotors. The upwash benefits are seen in the lead rotor results in both diamond and square configurations as there are lower rotor speeds required to maintain the same thrust requirement as the surrounding rotors.

\subsection{Moment Trim}

In the moment trim module, the thrust distribution of the rotors is modified in order to trim the vehicle moments while maintaining force trim. The moment module predicts the total residual moments of the vehicle about the all three axes and resolves the pitching moments to zero by proportionally adjusting the thrust of the lead and rear rotors. The quadrotor in this study has a lead rotor that spins counterclockwise.

Figure 5.11 shows the rolling, pitching, and yawing moments of the diamond and square configurations. For better plot clarity, similar forces and moments are grouped together into six categories of moment trends: moments due to rotor forces, rotor hub moments, torques, moments due to drag, lift, and weights. The moment produced by rotor forces is the sum of all rotor thrust and side forces, $F_{x}$ and $F_{y}$. The moment due to rotor hub moment is the sum of rolling moments, $M_{x}$, about the longitudinal axis and the sum of pitching moments, $M_{y}$, about the lateral axis with respect to each rotor hub. Moments due to rotor torque, $Q$, are plotted separately to the rotor rolling and pitching moments. Moments produced by drag include the parasitic drag, $D_{\text {par }}$, of all components, including the payload, plus induced drag of body, $D_{\text {ind,body }}$. The moment produced by lift is due to the downward lift generated by the body, $L_{b o d y}$, as modelled having a puck shape. The moment due to the weight, $W$, includes the moments produced by the weight of individual components. 

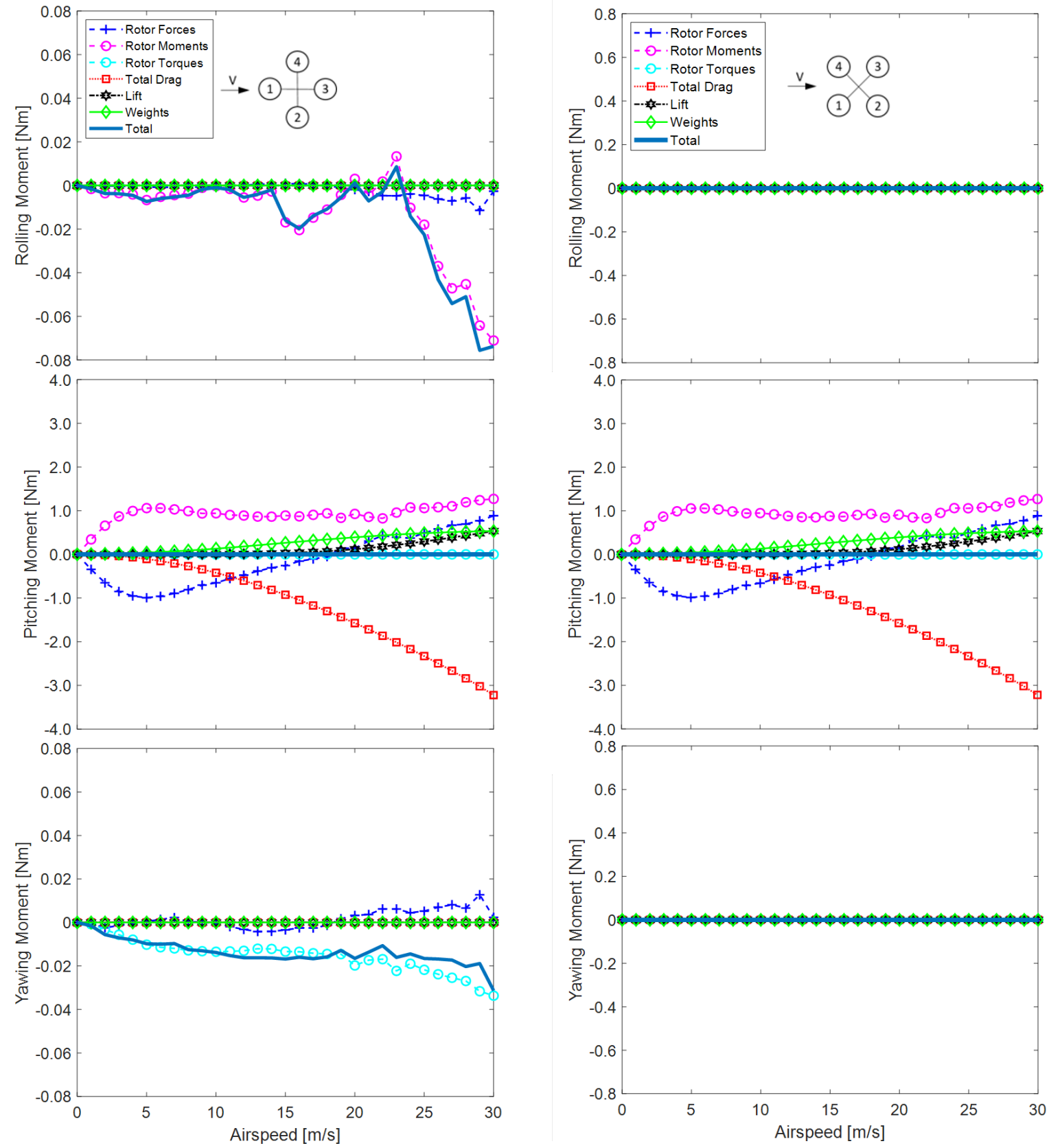

(a) Diamond

(b) Square

Figure 5.11: Residual moment components of the SkyRanger vehicle over a range of forward flight speeds. 
The moment trim prediction method gives insight into the moment contributions of each component. Notably, there is a crossover point where the pitching moment due to differential rotor forces, more specifically thrust, becomes positive around $18 \mathrm{~m} / \mathrm{s}$ in either configuration. This point reflects where the lead rotors begin to require more thrust than the rear rotors with increasing airspeed, as reflected in Fig. 5.1. This differential thrust behaviour is counter to the low speed behaviour and can cause significant issues for the control laws.

The pitching moment components are extremely similar between diamond and square configurations. Figure 5.12 shows the difference between diamond and square pitching moment results. The pitching moment components of total drag, weight and lift are the same due to the same pitch and freestream velocity conditions between configurations. The total rotor moments have slight differences, between $+/-0.02 \mathrm{Nm}$, due to different inflow conditions between rotors. The difference between rolling and yawing moments between configuration using the prediction method are more distinct than the pitching moment results.

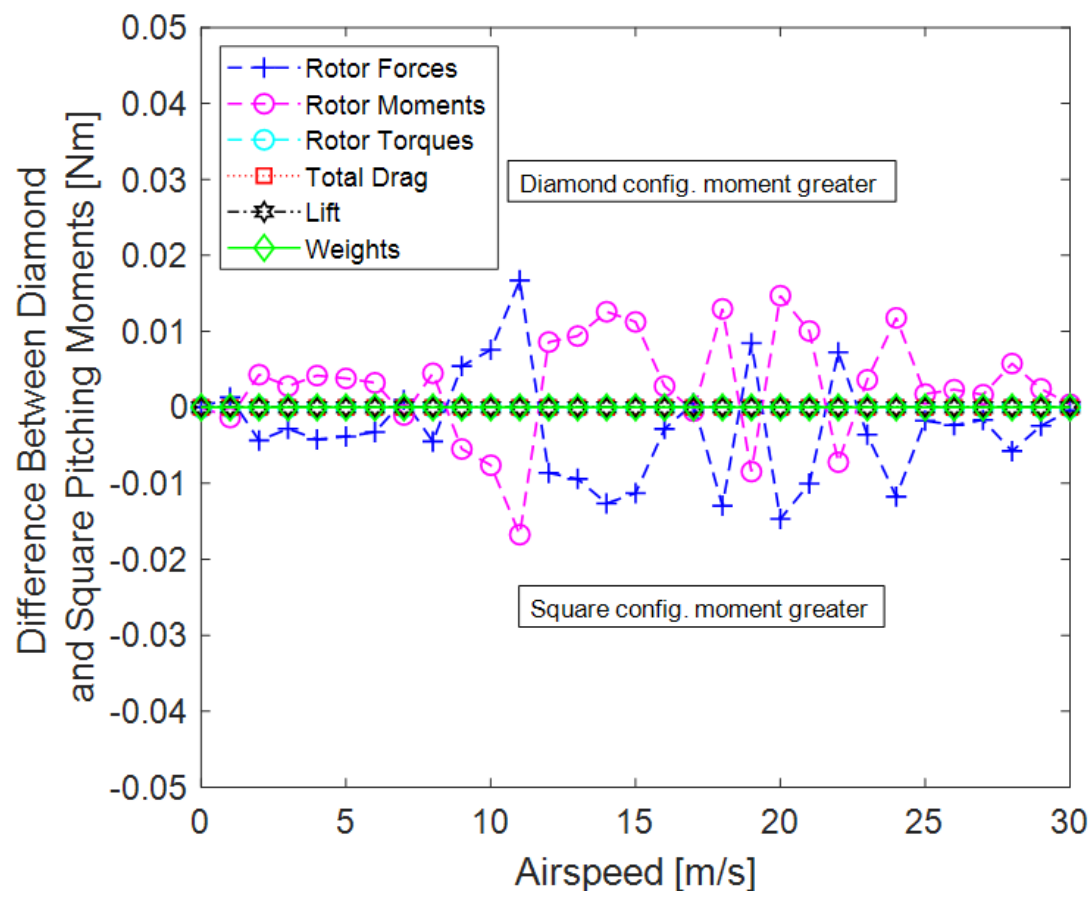

Figure 5.12: Difference between diamond and square pitching moment results. 
The rolling and yawing moment results, shown in Fig. 5.11, are zero for the square configuration. This is due to having rotor forces and hub moments of equal magnitude applied in opposite directions about the longitudinal axis. The results of the mirrored rotor forces and hub moments are due to averaging the rotor forces and moments within the performance prediction method and applying the forces and moments directly to the rotor hub. In reality, the rotors experience gradient forces and moments along the rotor span and about the azimuth station locations for a spinning rotor.

In diamond configuration, the rolling and yawing moments are due to the asymmetric force and moment distribution around the vehicle's longitudinal and vertical axes. The total rolling moment in diamond configuration is primarily due to the in rotor hub rolling moments, $M_{x}$. Generally, the sum of the negative rotor hub rolling moments of the side rotors is larger than the sum of the positive rotor hub rolling moments of the lead and rear rotors. The total residual yawing moment is a result of the sum of the moments produced by the lateral side force and rotor torques. Figure 5.13 shows an example of lead and rear rotors of side inplane forces of difference magnitudes resulting in a negative yawing moment about the vehicle's vertical axis. The direction of the total residual yawing moments in diamond configuration is determined by the difference of magnitudes of the rotor side forces, $F_{y}$, of the lead and rear rotors and the overall residual torques of each rotor. In diamond configuration, the lead rotor will typically have a lower assigned rotor thrust and the rear rotor a higher rotor thrust to maintain moment equilibrium. As a result, the rotors will have side inplane rotor forces, $F_{y}$, of differing magnitudes while having the same rotational direction. The differing $F_{y}$ magnitudes result in a yawing moment experienced by the multirotor vehicles. Similar to the yawing moment results shown in Fig. 5.11. Niemiec and Gandhi showed that yaw control is needed for diamond configuration, not square configuration, and that rotor side forces and hub torque are the only sources of yaw moments for a quadrotor vehicle [29]. The analysis shown in Ref. 29 did not trim for moments and all rotors were assigned the same rotor speed.

The rotor rotational direction does influence the rolling and yawing moments of a vehicle in square configuration, but the averaging method used in the multirotor vehicle performance model does not capture those effects. Reference 30 uses a higher-order potential flow method to determine sectional forces and moments of a rotor blade about the rotor plane. The method accounts for changing induced and interference velocities on each section of the rotor blade in forward flight. The thesis shows that in square configuration, depending on the direction of rotation of the lead rotors, the direction of the wake 
generated by the rotors will influence the rolling and yawing moments of the opposing rear rotor to the lead rotors. The method used for the herein presented results, however, does not capture any effects due to the rotational orientation.
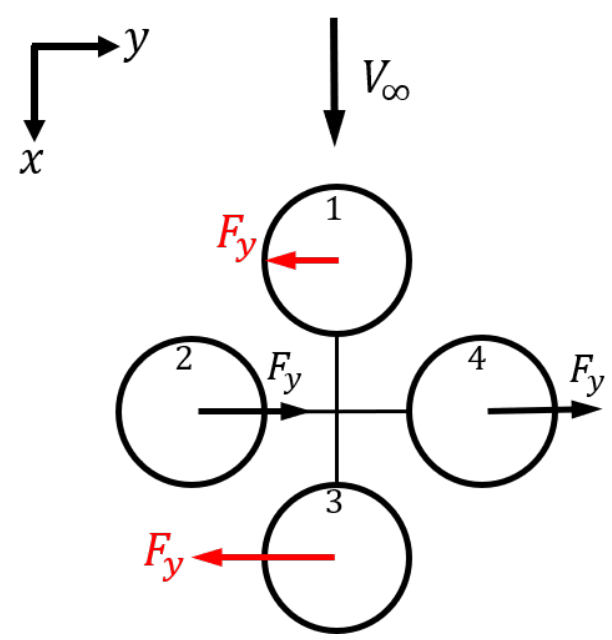

Figure 5.13: Example of negative yawing moment, $M_{z}$, applied to multirotor vehicle due to residual yawing moments generated by the inplane rotor forces, $F_{y}$. 


\section{CHAPTER 6}

\section{MASS OFFSET STUDY}

\subsection{Performance Comparison with Flight Test Results}

The multirotor vehicle performance prediction method is an analysis tool that allows multirotor designers to compare the predicted flight characteristics for different payloads. The mass offset study looks into the changes in flight characteristics due to an asymmetric centre of gravity location. In separate flight tests, washers were added to the arm of the lead rotor of a quadrotor vehicle in order to change the centre of gravity location of the vehicle. This simulates trying to explore the changes in flight characteristics due to a new payload and help the development of new robust control laws. Similar to the flight test comparison in Chapter 4, prediction results generated by using performance prediction method are compared to flight test results.

The offset testing was completed the same day as the validation tests, that are discussed in Chapter 4. The mass offset was achieved using up to $100 \mathrm{~g}$ of metal washers that were attached to the lead arm $0.6 \mathrm{~m}$ from the arm root, where the support arm and central body meet. For example, Fig. 6.1 shows a schematic of a mass, $m$, applied to the lead rotor of a quadrotor at a distance $x$ from the vehicle reference point. The environmental conditions changed by time of the mass-offset tests. The temperature increased from $8^{\circ} \mathrm{C}$ to $11^{\circ} \mathrm{C}$ and the wind picked up from $13 \mathrm{~km} / \mathrm{r}$ to $24 \mathrm{~km} / \mathrm{hr}$ West. The flight test procedure was the same as used during the validation testing, where the vehicle travelled at groundspeeds of $3,5,8$, and $10 \mathrm{~m} / \mathrm{s}$ in straight line flight paths into and with the wind. The flight test data of the tests were limited due to strong wind conditions. 


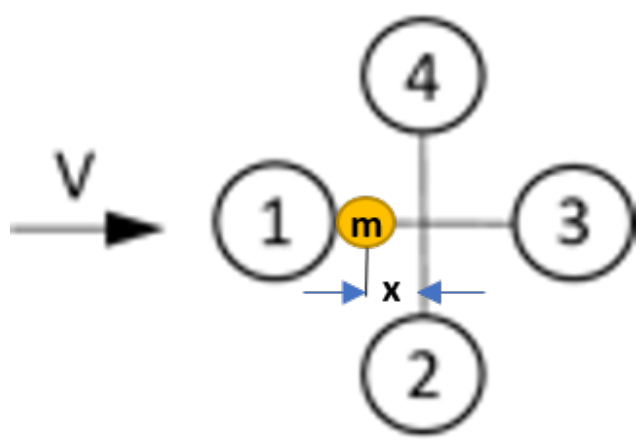

Figure 6.1: Quadrotor in diamond flight with mass, $\mathrm{m}$, attached to lead rotor support arm.

Figure 6.2a shows the comparison of predicted and recorded rotor speeds. Similar to the flight test results in Chapter 4, the relative trends of lead and rear rotor speed results show agreement, particularly near hover. The lead rotor flight test and prediction rotor speed results require higher rotor speeds than the rear and side rotors. A higher rotor speed for the lead rotor around hover is expected as the added mass is attached to the lead rotor arm and this the lead rotor requires higher thrust in order to maintain the moment equilibrium and a vehicle pitch attitude of $0^{\circ}$. In order to maintain force equilibrium, the rear rotor requires a decrease in thrust. At airspeeds greater than $5 \mathrm{~m} / \mathrm{s}$, the lead and rear rotor speeds are generally overpredicted, but the flight test and prediction rotor speeds do show similar differential rotor speed between lead and rear rotors. Fig. 6.2b shows the difference between lead and rear rotor speeds from the prediction and flight test. Where the trends between prediction and flight test results in this figure are similar, an important highlight is that both trends show the two crossover points where there is no difference between lead and rear rotor speeds results.

For control system development, it is beneficial to be able to predict the differential rotor speed requirements prior to flight tests. As different payload types are added to the multirotor vehicle, the control authority of the vehicle will change and maximum rotor speeds and power can be exceeded depending on payload mass and position. Being able to predict the rotor speed and power requirements for different payloads and payload positions gives insight into the limitations of centre of gravity locations in order to maintain control authority before any flight testing is conducted.

The side rotor predictions of Fig. 6.2a have little agreement with the flight test results. This discrepancy can be attributed to the simplistic moment trim model where only pitching moments are set 
to zero. The selected flight path was also in the direction towards a westward headwind, but a crosswind component or gust factor could have also affected flight test results.

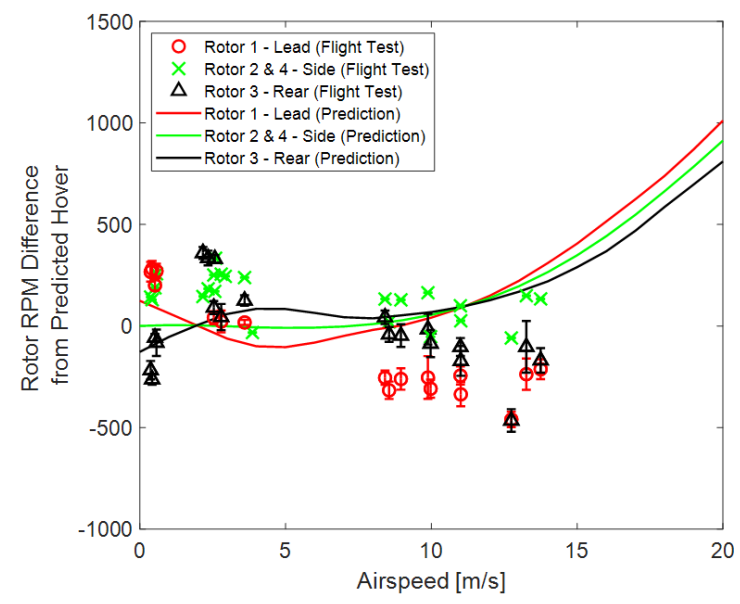

(a) Rotor speeds.

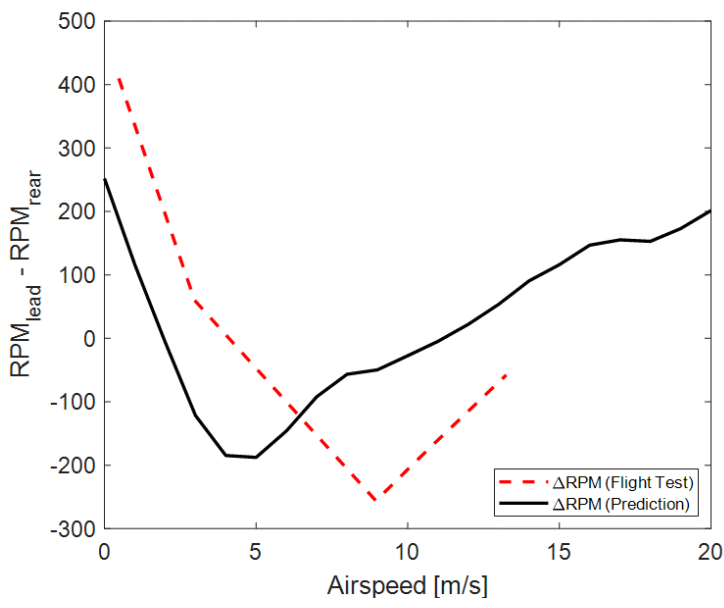

(b) Difference between lead and rear rotor speeds.

Figure 6.2: Rotor speed comparison between prediction and flight test results of a SkyRanger vehicle with a mass offset.

Total power is underpredicted at hover by about $100 \mathrm{~W}$, as shown in Fig. 6.3. Recall, that the total power measured from the vehicle is a combination of mechanical and electric power, whereas the predicted power is a combination of rotor and parasitic powers.

The pitch angles during the steady, level flight test are consistent with the baseline results from Chapter 4. Figure 6.4a and 6.4b show the pitch results and the difference between flight and prediction data, respectively. Five points were selected from the flight test data to compare with prediction results at the same airspeeds. There is a maximum pitch set on the SkyRanger vehicle set by Aeryon Labs Inc. Hence, at higher airspeeds, the predicted pitch attitude is higher than the limits set by Aeryon. At airspeeds below $10 \mathrm{~m} / \mathrm{s}$ the difference between predicted and recorded pitch angles are within $+/-1^{\circ}$. 


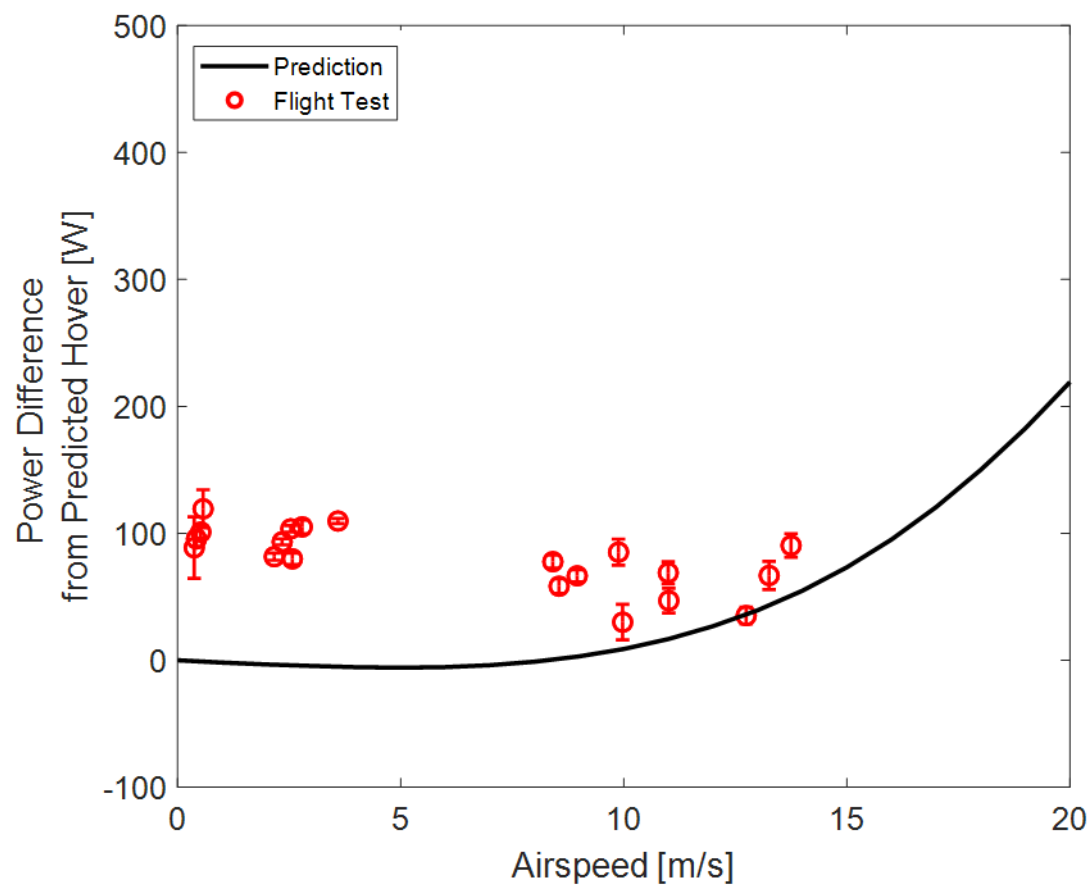

Figure 6.3: Comparison between flight test and prediction total vehicle power results for SkyRanger with a mass offset.

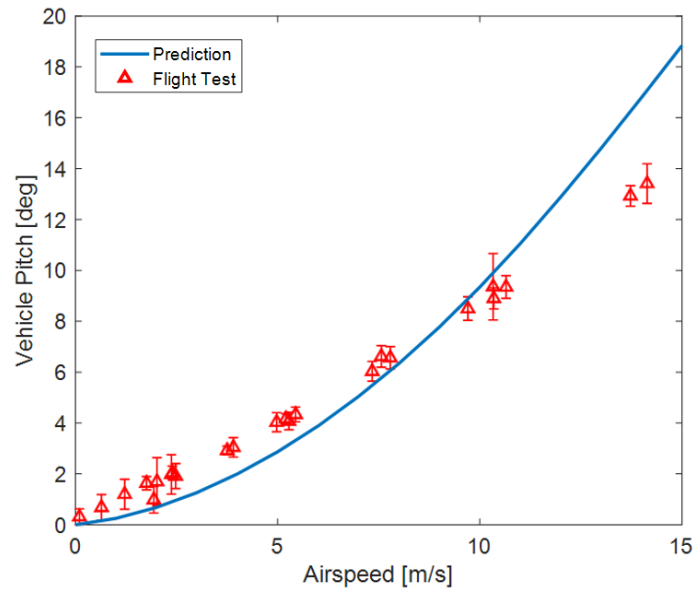

(a) Vehicle pitch.

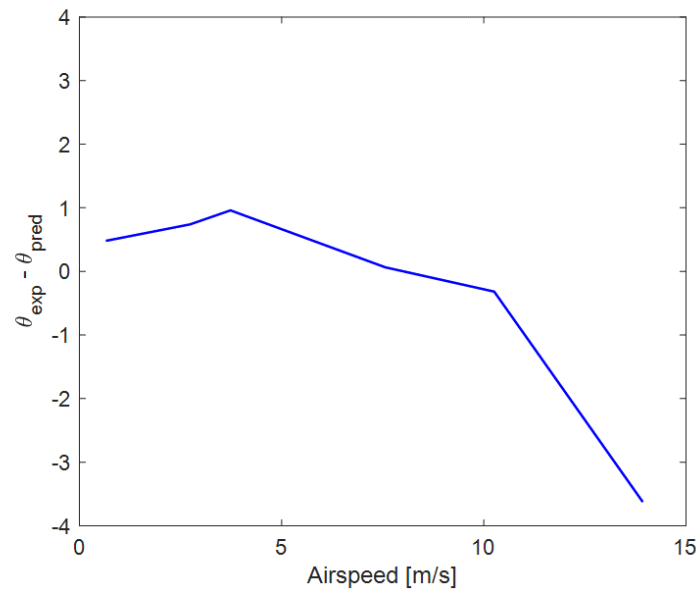

(b) $\theta_{F T}-\theta_{P}$

Figure 6.4: Comparison between flight test and prediction pitch results for a SkyRanger with a mass offset. 
The multirotor vehicle performance model can make computationally inexpensive predictions of flight characteristics of different vehicle designs with rotor speed results within $+/-500 \mathrm{rpm}$ and pitch results within $+/-2^{\circ}$ up to $20 \mathrm{~m} / \mathrm{s}$ airspeed. The computation time of the prediction results used in this study was under 5 minutes. The method is a useful too to help vehicle designers determine optional limits to mass offset masses and positions applied to the vehicle based on simulated rotor speeds and rotor power predicted by the multirotor vehicle performance method. An example is using a gripper-type device that can pick up or release a payload. Designers can then quickly asses the mass balance effects of between different object shapes and sizes the gripper-type payload can pick up and transport successfully prior to any flight testing taking place. 


\section{CHAPTER 7}

\section{CONCLUSIONS}

\subsection{Conclusions}

The performance prediction method is presented as a computationally efficient method that can be used to predict multirotor vehicle rotor speeds, rotor power, and pitch required for trimmed flight. The model can predict these performance characterists typically under 5 minutes on a standard laptop, depending on the input flight configuration and airspeed range. The fast computation time is attributed to the method using simple geometries and pre-generated rotor performance lookup tables to generate prediction results. The performance prediction model is an extended version of the method presented in Ref. [14. The extended version of the multirotor vehicle performance method introduces a moment trim model, a rotor speed prediction method, and a fuselage interference model. In addition, the input file is simplified allowing the user to enter basic geometries, atmospheric conditions, and component masses directly into a text file. Geometries generated by 3D modelling is not required, unlike other performance prediction methods like CFD.

The wind tunnel, flight test and mass offset performance comparison studies showed that the multirotor vehicle performance prediction method was a useful tool when predicting the changes in flight performance between different configurations and predicting the limitations of the flight envelope. The performance comparison of the 3DR Solo vehicle with the NASA Ames wind tunnel data showed that vehicle forces and pitching moment trends can be predicted even using estimated rotor and vehicle geometries. The rotor speed prediction results in the flight test study showed overlapping rotor speed 
results of the lead and rear rotors at flight speeds less than $5 \mathrm{~m} / \mathrm{s}$. For all flight speeds, the differences between lead and rear rotor speeds of the predicted results also reflected the change in rotor speeds required to maintain trimmed flight. The lead and rear rotor speed results show that the application of the moment trim model provided rotor speed estimates that reflect the trends of steady level flight test results. The mass offset study showed similar results to the flight where a mass of washers were attached to the lead rotor of a vehicle in diamond flight configuration. The predicted rotor speed distribution for the mass offset study, particularly at hover where the lead rotor required higher rotor speeds than the baseline flight test and the rear rotor required a lower rotor speed, reflected the the rotor speed distribution for a vehicle with an asymmetric mass loading, which simulates different payload loading.

The predicted performance results demonstrated that the method is a useful tool to study the extreme loading factors of interchangeable payloads. Extreme loading factors include attaching a heavy payload, attaching a payload with an asymmetric load distribution, or attaching differently shaped payload affecting the drag profile of the vehicle. The prediction method capabilities can determine the limitations of certain vehicle designs and payload configurations. For instance, adding a payload that is too heavy or provides asymmetric loading on the vehicle may result in the rotors exceeding power and rotor speed limits. The performance prediction method can be used to checking these performance limitations prior to flight testing.

\subsection{Future Development}

There are a number of areas of development of which the multirotor vehicle performance model can be improved. Future development includes:

1. Developing climbing and descending flight performance prediction methods,

2. Improving the trim routine to include trim rolling and yawing moments,

3. Incorporating wings and other lifting surfaces into the multirotor vehicle designs and include lifting forces into trim model,

4. Developing an optimization scheme for explore different vehicle configurations,

5. Exploring high speed flight greater than $20 \mathrm{~m} / \mathrm{s}$ and the performance prediction methods validity in this region. 


\section{Appendix A}

\section{VEHICLE INPUT DATA}

\section{A.1 3DR Solo Input Parameters}

3DR Solo component geometry input

\begin{tabular}{|l|c|}
\hline Arm length & 0.124 \\
\hline Arm radius & 0.013 \\
\hline Body height & 0.248 \\
\hline Body radius & 0.053 \\
\hline Leg length & 0.156 \\
\hline Leg radius & 0.200 \\
\hline Leg centre radius from origin & 0.100 \\
\hline Leg centre height from origin & 0.000 \\
\hline Payload length & 0.000 \\
\hline Payload radius & 0.156 \\
\hline Payload height from origin to mid axis & 0.047 \\
\hline Motor height & 0.010 \\
\hline Motor radius & 0.055 \\
\hline Rotor hub height from mid motor to mid rotor hub & 0.100 \\
\hline CG height from origin & 0.028 \\
\hline
\end{tabular}


3DR Solo component mass input

\begin{tabular}{|l|l|}
\hline Motor mass & 0.086 \\
\hline Arm mass & 0.057 \\
\hline Leg mass & 0.057 \\
\hline Payload mass & 0.000 \\
\hline Body mass & 0.700 \\
\hline Total vehicle mass & 1.500 \\
\hline
\end{tabular}

\section{A.2 GRCSP 10x8 Rotor Geometry Input}

GRCSP 10x8 rotor geometry inputs

\begin{tabular}{|l|l|l|l|l|l|l|l|}
\hline Station & Radius & $\mathrm{r} / \mathrm{R}$ & Chord & c_R & Beta & Airfoil & Blades \\
\hline 1 & 0.01905 & 0.15 & 0.016383 & 0.129 & 41.89 & naca0012' & 2 \\
\hline 2 & 0.0254 & 0.2 & 0.02032 & 0.16 & 48.64 & naca0012' & 2 \\
\hline 3 & 0.03175 & 0.25 & 0.023495 & 0.185 & 48.91 & naca0012' & 2 \\
\hline 4 & 0.0381 & 0.3 & 0.024892 & 0.196 & 44.58 & clark_x' & 2 \\
\hline 5 & 0.04445 & 0.35 & 0.025527 & 0.201 & 40.1 & clark_x' & 2 \\
\hline 6 & 0.0508 & 0.4 & 0.025654 & 0.202 & 36.16 & clark_x' & 2 \\
\hline 7 & 0.05715 & 0.45 & 0.0254 & 0.2 & 32.76 & clark_x' & 2 \\
\hline 8 & 0.0635 & 0.5 & 0.024892 & 0.196 & 29.79 & clark_x' & 2 \\
\hline 9 & 0.06985 & 0.55 & 0.02413 & 0.19 & 27.18 & clark_x' & 2 \\
\hline 10 & 0.0762 & 0.6 & 0.023241 & 0.183 & 24.92 & clark_x' & 2 \\
\hline 11 & 0.08255 & 0.65 & 0.022098 & 0.174 & 22.97 & clark_x' & 2 \\
\hline 12 & 0.0889 & 0.7 & 0.020828 & 0.164 & 21.25 & clark_x' & 2 \\
\hline 13 & 0.09525 & 0.75 & 0.019304 & 0.152 & 19.73 & clark_x' & 2 \\
\hline 14 & 0.1016 & 0.8 & 0.017653 & 0.139 & 18.32 & clark_x' & 2 \\
\hline 15 & 0.10795 & 0.85 & 0.015494 & 0.122 & 17.01 & clark_x' & 2 \\
\hline 16 & 0.1143 & 0.9 & 0.012827 & 0.101 & 15.83 & clark_x' & 2 \\
\hline 17 & 0.12065 & 0.95 & 0.008509 & 0.067 & 14.68 & clark_x' & 2 \\
\hline 18 & 0.127 & 1 & 0.004191 & 0.033 & 13.53 & clark_x' & 2 \\
\hline
\end{tabular}




\section{A.3 SkyRanger Input Parameters}

SkyRanger rotor parameter input

\begin{tabular}{|l|l|}
\hline Rotor type & T-Motor \\
\hline Number of rotors & 4 \\
\hline Rotor diameter $(\mathrm{m})$ & 0.4064 \\
\hline Number of blades & 2 \\
\hline
\end{tabular}

SkyRanger component geometry input in meters

\begin{tabular}{|l|c|}
\hline Arm length & 0.200 \\
\hline Arm radius & 0.010 \\
\hline Body height & 0.145 \\
\hline Body radius & 0.100 \\
\hline Leg length & 0.295 \\
\hline Leg radius & 0.010 \\
\hline Leg centre radius from origin & 0.179 \\
\hline Leg centre height from origin & 0.160 \\
\hline Payload length & 0.165 \\
\hline Payload radius & 0.045 \\
\hline Payload height from origin to mid axis & 0.190 \\
\hline Motor height & 0.030 \\
\hline Motor radius & 0.020 \\
\hline Rotor hub height from mid motor to mid rotor hub & 0.031 \\
\hline CG height from origin & 0.028 \\
\hline
\end{tabular}

SkyRanger component mass input in kilograms

\begin{tabular}{|l|l|}
\hline Motor mass & 0.085 \\
\hline Arm mass & 0.063 \\
\hline Leg mass & 0.029 \\
\hline Payload mass & 0.686 \\
\hline Body mass & 2.000 \\
\hline Total vehicle mass & 3.336 \\
\hline
\end{tabular}




\section{A.4 T-Motor Rotor Geometry Input}

T-Motor 18x6.1 rotor geometry inputs

\begin{tabular}{|c|c|c|c|c|c|c|c|}
\hline Station & Radius & $\mathrm{r} / \mathrm{R}$ & Chord & $\mathrm{c} / \mathrm{R}$ & Beta & Airfoil & Blades \\
\hline 1 & 0.01143 & 0.05 & 0.019428 & 0.0849868766 & 1.209 & airfoil5' & 2 \\
\hline 2 & 0.02286 & 0.1 & 0.019862 & 0.0868853893 & 1.209 & airfoil5' & 2 \\
\hline 3 & 0.03429 & 0.15 & 0.025314 & 0.1107349081 & 15.763 & airfoil5' & 2 \\
\hline 4 & 0.04572 & 0.2 & 0.033232 & 0.1453718285 & 22.586 & airfoil5' & 2 \\
\hline 5 & 0.05715 & 0.25 & 0.038645 & 0.1690507437 & 21.375 & airfoil5' & 2 \\
\hline 6 & 0.06858 & 0.3 & 0.042025 & 0.1838363955 & 19.727 & airfoil10' & 2 \\
\hline 7 & 0.08001 & 0.35 & 0.044301 & 0.1937926509 & 17.023 & airfoil10' & 2 \\
\hline 8 & 0.09144 & 0.4 & 0.04382 & 0.1916885389 & 15.476 & airfoil10' & 2 \\
\hline 9 & 0.10287 & 0.45 & 0.043009 & 0.1881408574 & 13.973 & airfoil10' & 2 \\
\hline 10 & 0.1143 & 0.5 & 0.041574 & 0.1818635171 & 12.472 & airfoil10' & 2 \\
\hline 11 & 0.12573 & 0.55 & 0.040027 & 0.175096238 & 11.9 & airfoil10' & 2 \\
\hline 12 & 0.13716 & 0.6 & 0.038589 & 0.1688057743 & 10.937 & airfoil10' & 2 \\
\hline 13 & 0.14859 & 0.65 & 0.036457 & 0.1594794401 & 10.536 & airfoil15' & 2 \\
\hline 14 & 0.16002 & 0.7 & 0.034285 & 0.1499781277 & 9.683 & airfoil15' & 2 \\
\hline 15 & 0.17145 & 0.75 & 0.032684 & 0.1429746282 & 9.268 & airfoil15' & 2 \\
\hline 16 & 0.18288 & 0.8 & 0.030497 & 0.133407699 & 8.885 & airfoil15' & 2 \\
\hline 17 & 0.19431 & 0.85 & 0.027804 & 0.1216272966 & 8.336 & airfoil15' & 2 \\
\hline 18 & 0.20574 & 0.9 & 0.024821 & 0.1085783027 & 7.352 & airfoil15 & 2 \\
\hline 19 & 0.21717 & 0.95 & 0.021075 & 0.092191601 & 7.123 & airfoil15' & 2 \\
\hline 20 & 0.2286 & 1 & 0.009098 & 0.0397987752 & 7.526 & airfoil15' & 2 \\
\hline
\end{tabular}




\section{Appendix B}

\section{MANUAL: FAST MULTIROTOR PERFORMANCE PREDICTION PROGRAM}

The fast multirotor performance prediction (FMPP) method is a series of modules that predict rotor speeds and power required for steady, straight and level flight. The method finds force and moment trim solutions for a multirotor vehicle over a range of flight speeds. This method is a MATLAB program that can determine steady, level trim solutions of a multirotor vehicle. This manual includes FMPP setup instructions and descriptions of output variables. 


\section{B.1 FMPP Startup}

\section{B.1.1 File and Folder Startup}

The program folder, Fig. ?? needs to have the following files and folder: main file, all of the functions, the input folder, and the rotor folder. It is important that the input and rotor folders start with lower case letters.

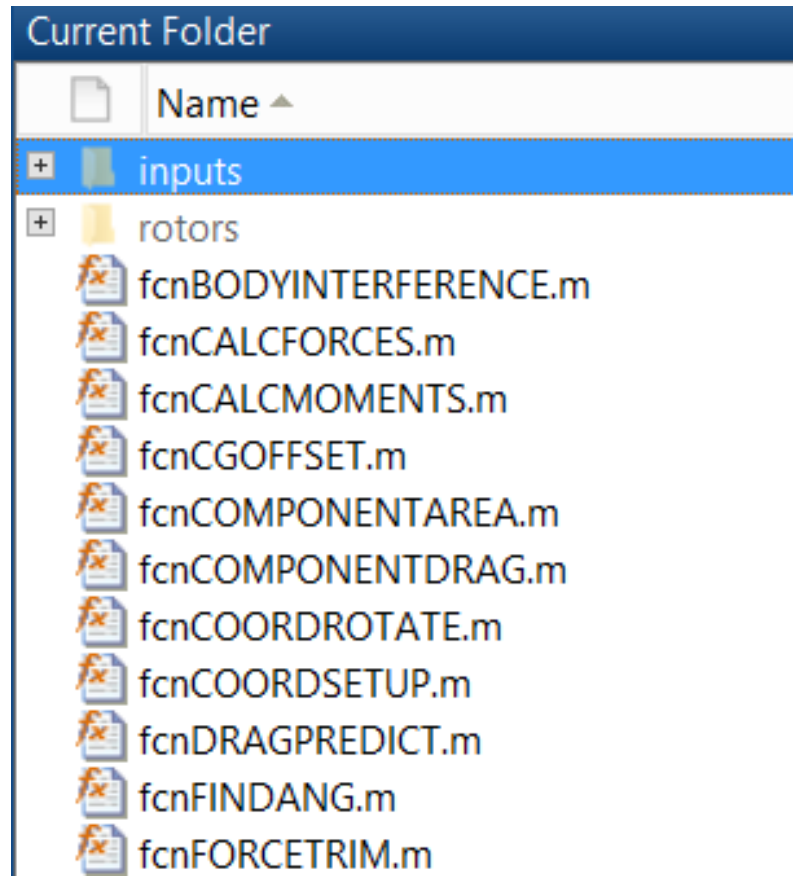

File organization. 


\section{B.1.2 Input File Selection}

The text files in the input folder can have any name. The exact file name is entered into the 5 th line of the Main_FMPP program as the strFILE variable (Fig. ??). All input files must have the same format as shown in Fig. ??

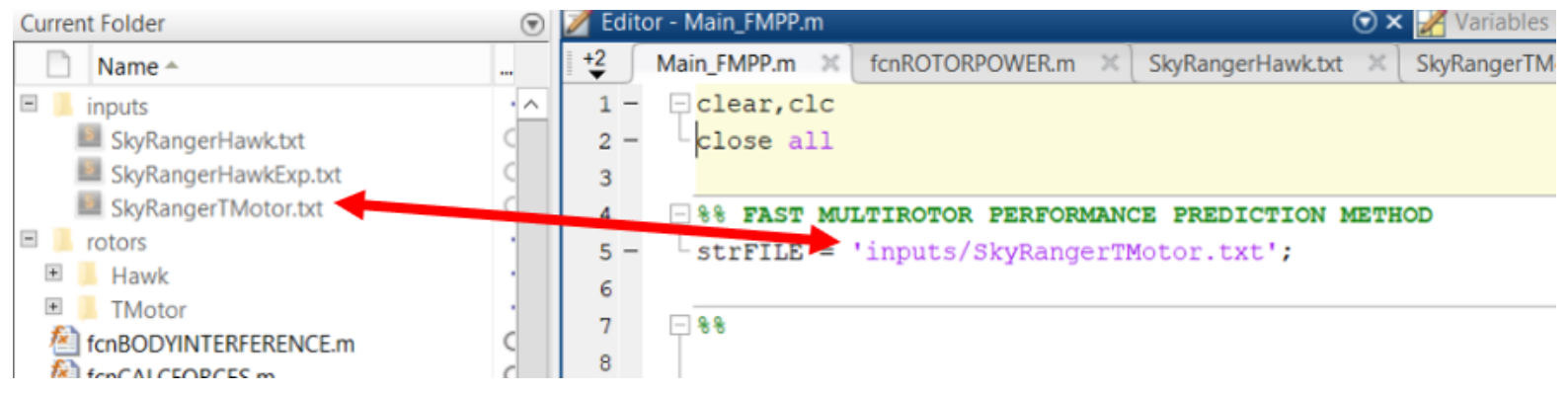

Input file name entry. 


\section{B.1.3 Input File Setup}

Figure?? shows the layout of the input file and sample geometries for the components of the SkyRanger vehicle components using Hawk rotors.

Notes:

- Please note that the program uses equal, number (\#), quotation marks, and colons (:) as special identifiers

- The number directly after the equal sign will be used as the variable value.

- Anything typed after the number will not be considered as the variable value (see Lines 15, 34, and 61 in Fig. ??).

- Comments can be written anywhere. 


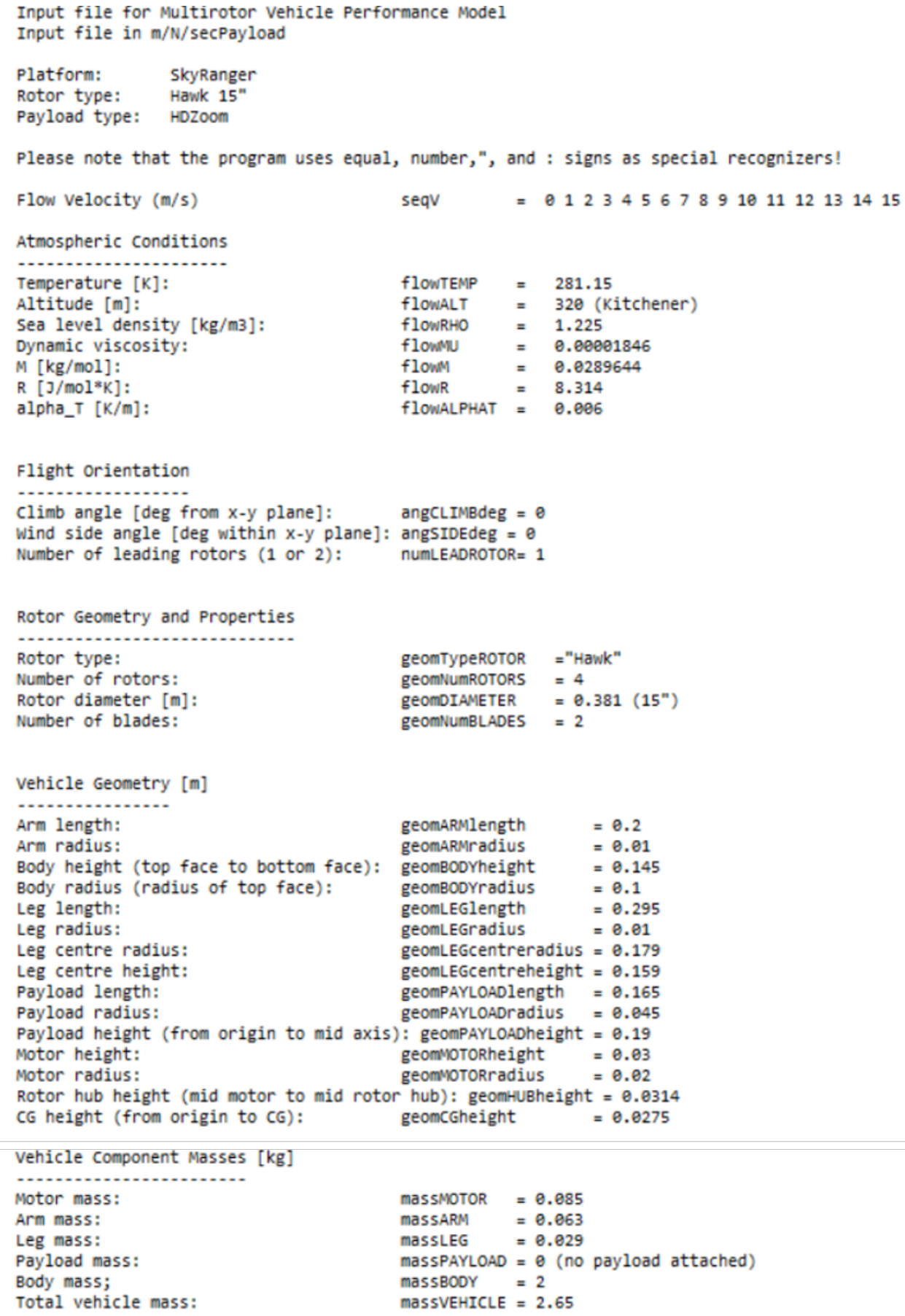

Sample input file for FMPP.

The following sections describe the input file entries line by line: 
Flow Velocity:

- Line 10: [vector] Indicate forward velocity range by entering each number in the range including spaces

Atmospheric conditions: [values] Properties of the day

- Line 14: [value] Temperature in Kelvin

- Line 15: [value] Altitude in meters

- Line 16: [value] Sea level density in $[\mathrm{kg} / \mathrm{m} 3]$

- Line 17: [value] Dynamic viscosity in $\left[\mathrm{N} \mathrm{s} / \mathrm{m}^{2}\right]$ at temperature

- Line 18: [value] Molar mass of air in $[\mathrm{kg} / \mathrm{mol}]$

- Line 19: [value] Gas constant in $[\mathrm{J} / \mathrm{mol} * \mathrm{k}]$

- Line 20: [value] Temperature coefficient in $[\mathrm{K} / \mathrm{m}]$

Flight Orientation:

- Line 25: [value] Climb angle and Line 26: Wind side angle are unused in prediction method and currently serve as placeholders.

- Line 27: [value 1 or 2 only] One leading rotor for a quadrotor is + configuration; Two leading rotors for a quadrotor is $\mathrm{x}$.

Rotor Geometry and Properties:

- Line 32: [string] Rotor type is the name of the folder that contains the BEMT generated tables. Do not put space between first quotation symbol and equal sign.

- Line 33: [value] Number of rotors on vehicle

- Line 34: [value] Rotor diameter in meters

- Line 35: [value] Number of blades of selected rotor

Vehicle Component Masses: [value] mass of component in kilograms 
- Line 58: [value] Mass of one rotor

- Line 59: [value] Mass of one support arm

- Line 60: [value] Mass of one leg

- Line 61: [value] Mass of payload

- Line 62: [value] Mass of central body

- Line 63: [value] Total mass of vehicle

Vehicle Geometry: [value] distance in meters (See Figs. ?? and ?? for diagrams of component geometry locations)

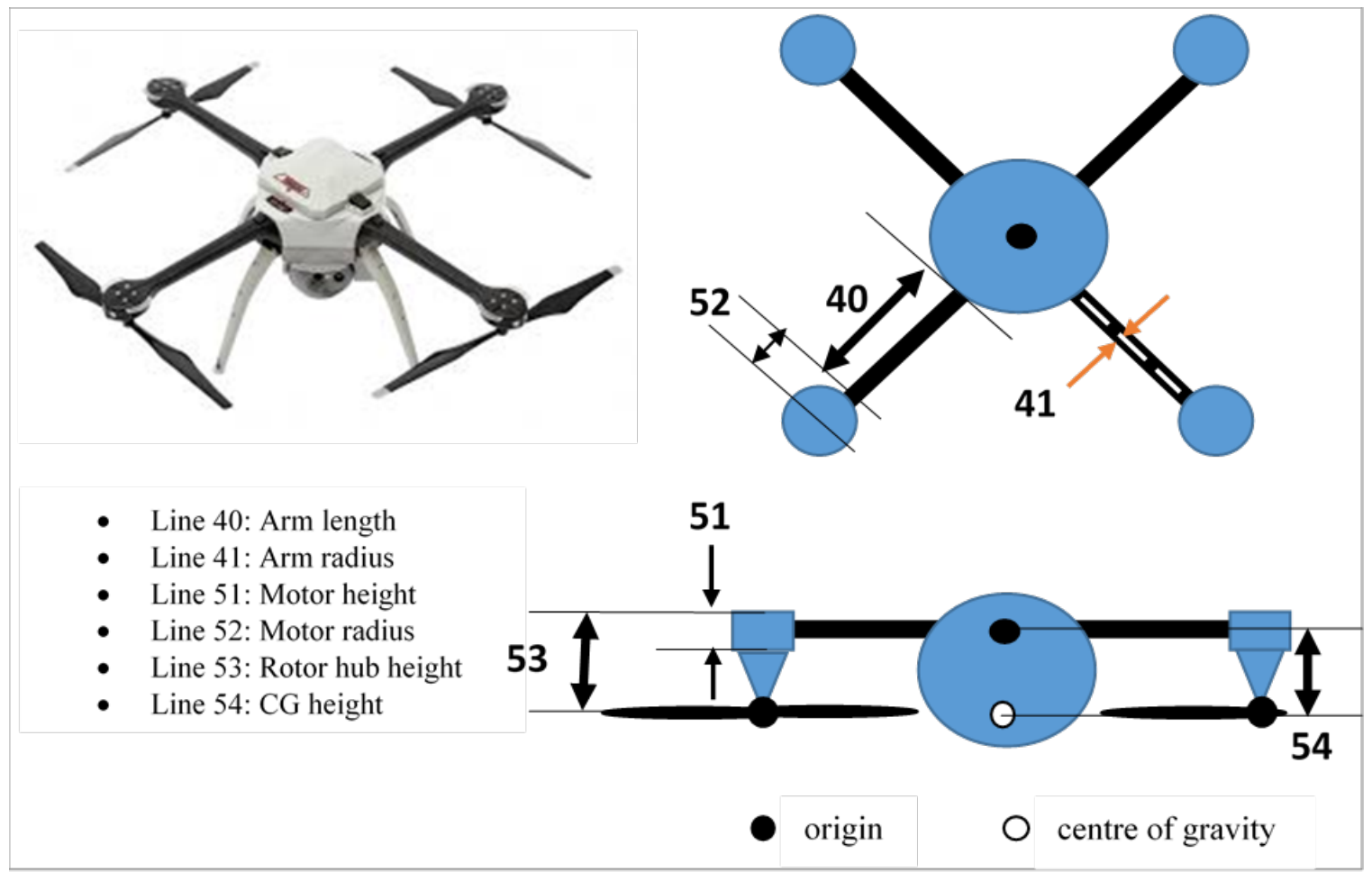

Input geometries of arm, motor, rotor rub, and location of centre of gravity from origin. 


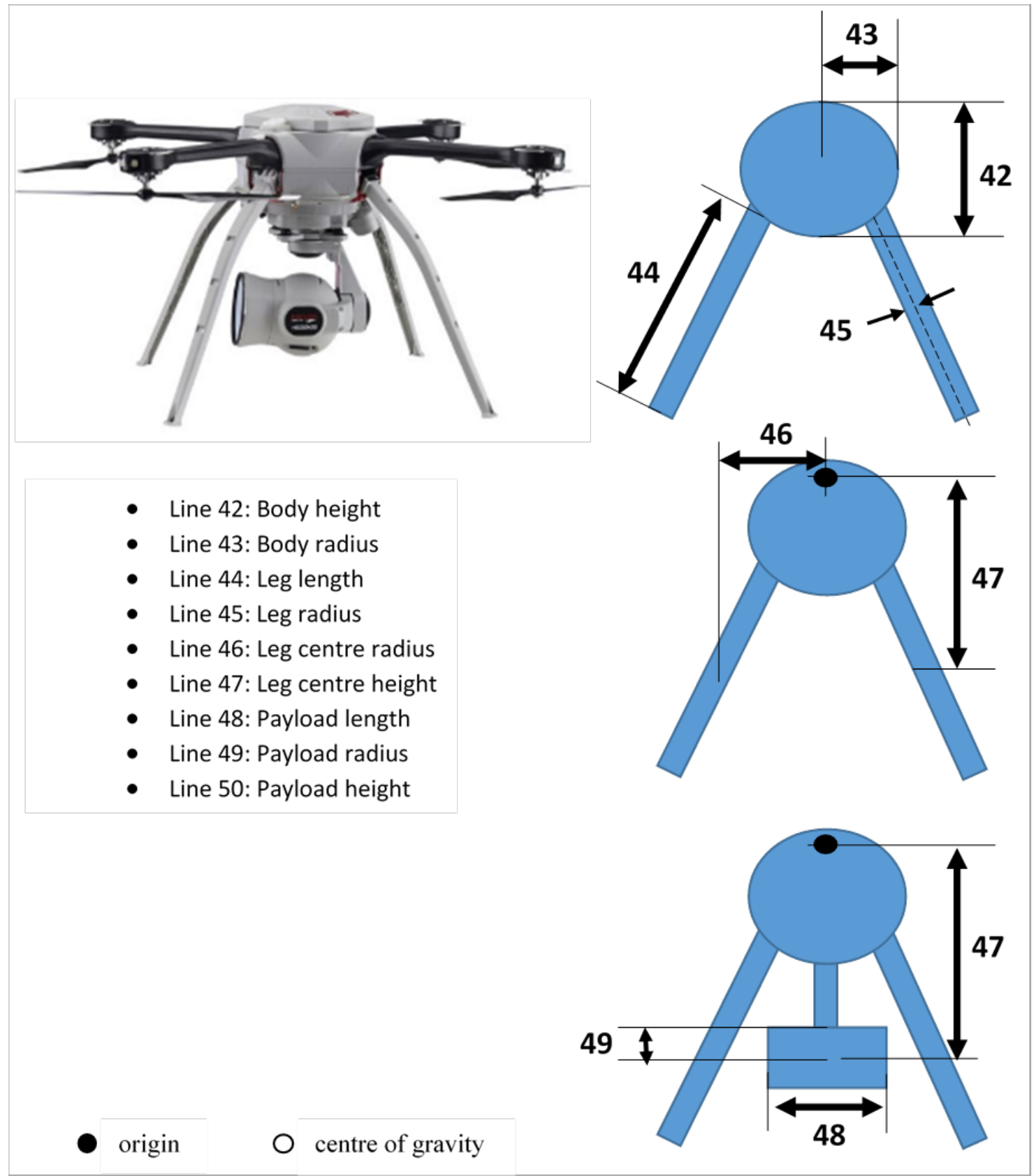

Input geometries of body, landing gear (leg), and payload. 


\section{B.1.4 Rotor Folder Setup}

Figure ?? shows the structure of the rotor folder using Hawk rotor and T-Motor rotor examples. The rotor name entered in Line 32 of the input text file must be the same name as the folder within the rotor folder. The naming convention of the rotor performance file names is:

pitch angle _ rotor name

If the pitch angle, in degrees, is negative, the lowercase letter $\mathrm{n}$ is placed as the first character of the file name.

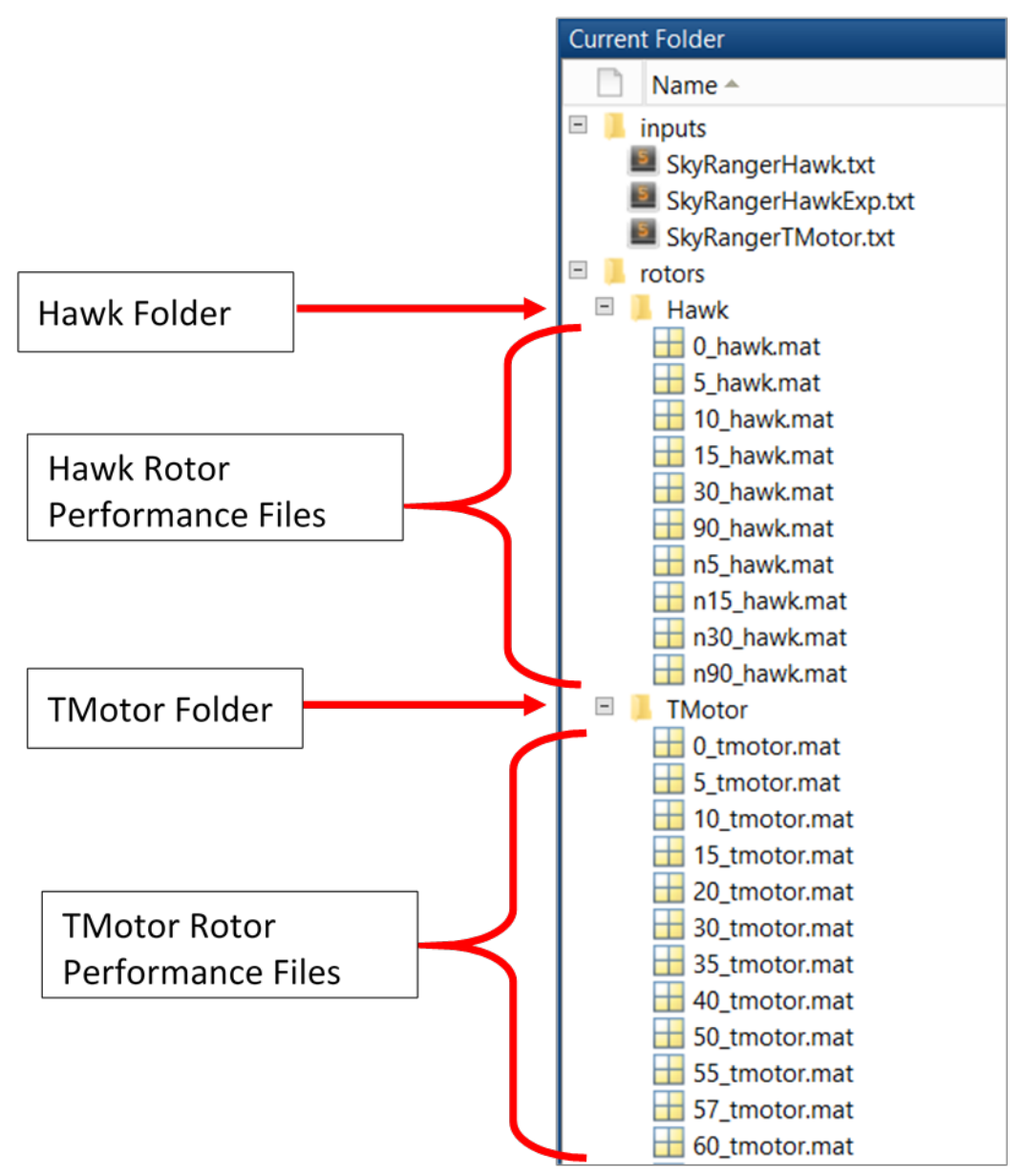

Rotor folder and file organization.

Figure ?? shows an example of the MATLAB table format for the file named 5_tmotor.mat. The workplace variable will have a different name as the file name because MATLAB does not allow for 
numbers to start a variable name (see tmotor_5deg table name in ??).

Note:

- Columns containing _rho such as Thrust_rho indicated that the values in the columns are force and moments divided by density.

\begin{tabular}{|c|c|c|c|c|c|c|c|c|c|c|}
\hline \multicolumn{11}{|c|}{ tmotor_5deg $x$} \\
\hline \multicolumn{11}{|c|}{ 田 $164 \times 10$ table } \\
\hline & $\begin{array}{l}1 \\
q\end{array}$ & ${ }_{\text {mu_inf }}^{2}$ & $\begin{array}{c}3 \\
\text { Thrust_rno }\end{array}$ & $\begin{array}{c}4 \\
\text { RPM }\end{array}$ & $\begin{array}{c}5 \\
C P\end{array}$ & $\begin{array}{c}6 \\
\text { Qrho }\end{array}$ & $\begin{array}{c}7 \\
\text { Px_rno }\end{array}$ & $\begin{array}{c}8 \\
\text { Mx_rno }\end{array}$ & $\begin{array}{c}9 \\
\text { Py_rho }\end{array}$ & $\begin{array}{c}10 \\
\text { My_rho }\end{array}$ \\
\hline 1 & 0 & 0 & 3.6174 & 2000 & $9.3641 e-04$ & 0.0806 & $-4.1539 e-17$ & $3.7763 e-18$ & $-6.0420 \mathrm{e}-17$ & $-6.0420 \mathrm{e}-17$ \\
\hline 2 & 0.6125 & 0.0209 & 3.6425 & 2000 & $9.2061 \mathrm{e}-04$ & 0.0792 & -0.0258 & -0.0327 & -0.0058 & -0.0645 \\
\hline 3 & 2.4500 & 0.0418 & 3.7648 & 2000 & $8.7706 \mathrm{e}-04$ & 0.0755 & -0.0497 & -0.0625 & -0.0031 & -0.1131 \\
\hline 4 & 5.5125 & 0.0627 & 3.9230 & 2000 & $8.3261 e-04$ & 0.0716 & -0.0724 & -0.0927 & 0.0044 & -0.1463 \\
\hline 5 & 9.8000 & 0.0835 & 4.0964 & 2000 & $8.0615 e-04$ & 0.0694 & -0.0926 & -0.1227 & 0.0122 & -0.1613 \\
\hline 6 & 15.3125 & 0.1044 & 4.2776 & 2000 & $7.9624 \mathrm{e}-04$ & 0.0685 & -0.1126 & -0.1519 & 0.0198 & -0.1639 \\
\hline 7 & 22.0500 & 0.1253 & 4.4474 & 2000 & $7.9385 e-04$ & 0.0683 & -0.1269 & -0.1807 & 0.0275 & -0.1601 \\
\hline 8 & 30.0125 & 0.1462 & 4.5990 & 2000 & $8.0172 e-04$ & 0.0690 & -0.1493 & -0.2064 & 0.0318 & -0.1532 \\
\hline
\end{tabular}

Sample format of 5_tmotor performance lookup table.

The general format for the rotor performance lookup table is shown in Figs. [?? and ?? Figure ?? has the file name a1_rotorname.mat, where a1 is the pitch angle in degrees. The file is organized by sequential dynamic pressure values, q1, q2, q3, etc. for the same rotor speed. The dynamic pressure values are then repeated for the next rotor speed set. Figure?? has the same table format for a second file name a2_rotorname.mat, where a2 is a different pitch angle than a1. The blank cells in the tables are the force, moment, and power values associated with the dynamic pressure and rotor speed of that line. 


\begin{tabular}{|l|l|l|l|l|l|l|l|l|l|}
\hline q & mu_inf & Thrust_rho & RPM & CP & Q_rho & Fx_rho & Mx_rho & Fy_rho & My_rho \\
\hline q1 & & & 2000 & & & & & & \\
\hline q2 & & & & & & & & & \\
\hline $\mathrm{q} 3$ & & & & & & & & & \\
\hline $\mathrm{q} 4$ & & & & & & & & & \\
\hline$\ldots$ & & & & & & & & & \\
\hline$\ldots$ & & & & & & & & & \\
\hline $\mathrm{q} 1$ & & & 3000 & & & & & & \\
\hline $\mathrm{q} 2$ & & & & & & & & & \\
\hline$\ldots--$ & & & & & & & & & \\
\hline--- & & & & & & & & & \\
\hline $\mathrm{q} 1$ & & & 4000 & & & & & & \\
\hline $\mathrm{q} 2$ & & & & & & & & & \\
\hline$\ldots$ & & & & & & & & & \\
\hline$\ldots$ & & & & & & & & & \\
\hline
\end{tabular}

Example file name - a1_rotornamt.mat.

\begin{tabular}{|l|l|l|l|l|l|l|l|l|l|}
\hline q & mu_inf & Thrust_rho & RPM & CP & Q_rho & Fx_rho & Mx_rho & Fy_rho & My_rho \\
\hline q1 & & & 2000 & & & & & & \\
\hline q2 & & & & & & & & & \\
\hline$\ldots$ & & & & & & & & & \\
\hline$\ldots$ & & & & & & & & & \\
\hline q1 & & & 3000 & & & & & & \\
\hline q2 & & & & & & & & & \\
\hline$\ldots$ & & & & & & & & & \\
\hline$\ldots$ & & & & & & & & & \\
\hline
\end{tabular}

Example file name - a2_rotornamt.mat. 


\section{B.1.5 Setting Up Lookup Tables Using BEMT Rotor Analysis Code}

The prediction lookup tables provided in the rotor folder were generated using the blade-element momentum theory model by Tim Carroll [17. Load up the BEMT_analysis file to setup the performance sweep inputs for the desired rotor.

To generate rotor files of the correct format used in the FMPP code, changes were made to the Performance_sweep.m file in the BEMT Rotor Analysis Code. The Performance_sweep file is accessed in the BEMT Rotor Analysis Code in the following sequence:

1. Rotor Analysis Code

2. BEMT Module

3. Performance Sweeps

4. Performance_sweep.m

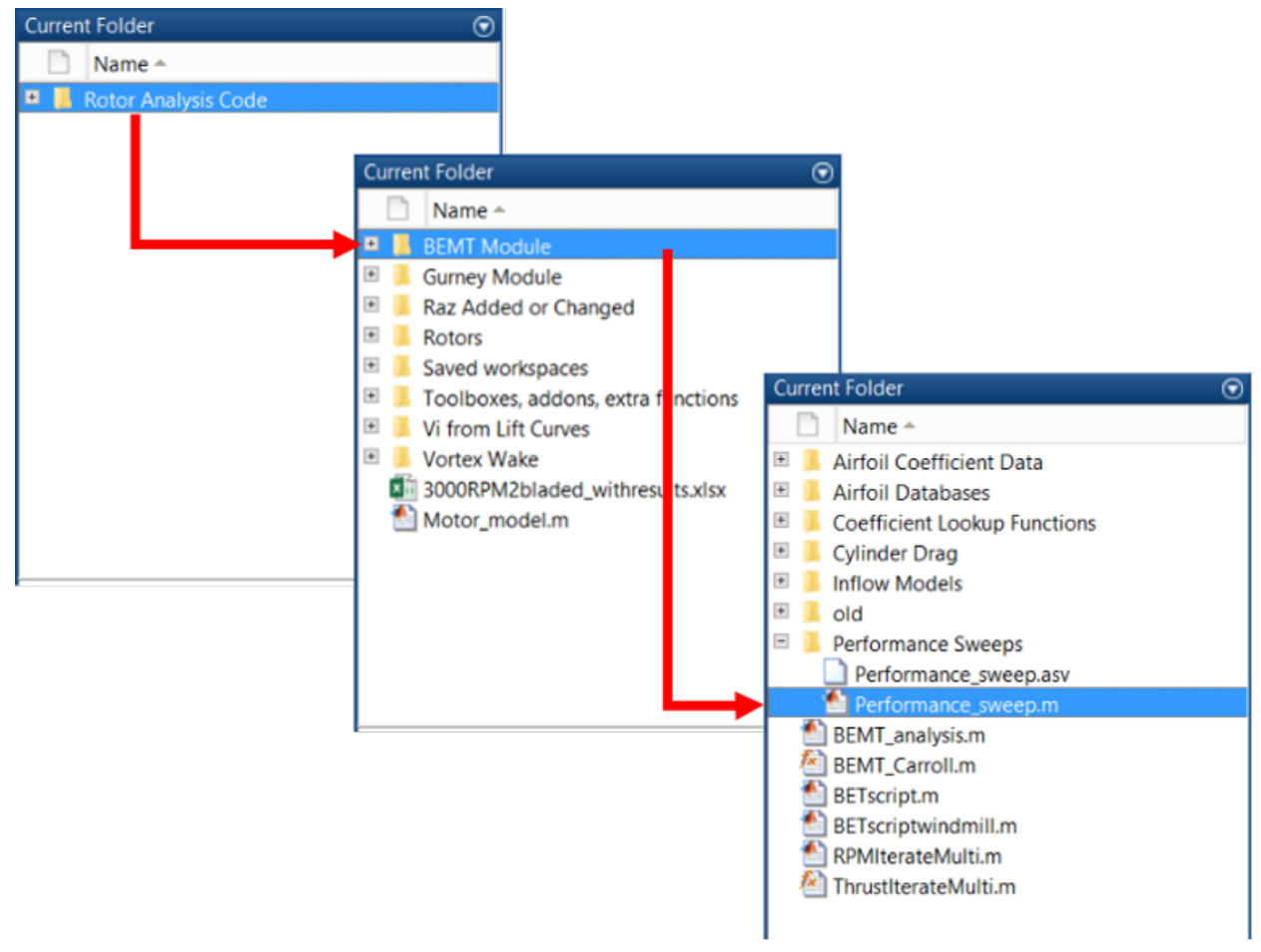

Folder tree to access Performance_sweep.mat file. 
Figure?? shows the lines of code modified in the BEMT model under case 1 within the Performance_sweep.mat file to accommodate the required FMPP table format. The modifications include adding loops for the rotor speed variable, rpm, as highlighted by the box labelled 1. Box 2 shows the order of variables for the lookup table with the air density divided from each of the rotor force and moment results. Finally, box 3 shows the workspace variable name given to the lookup table data with the corresponding table variable names.

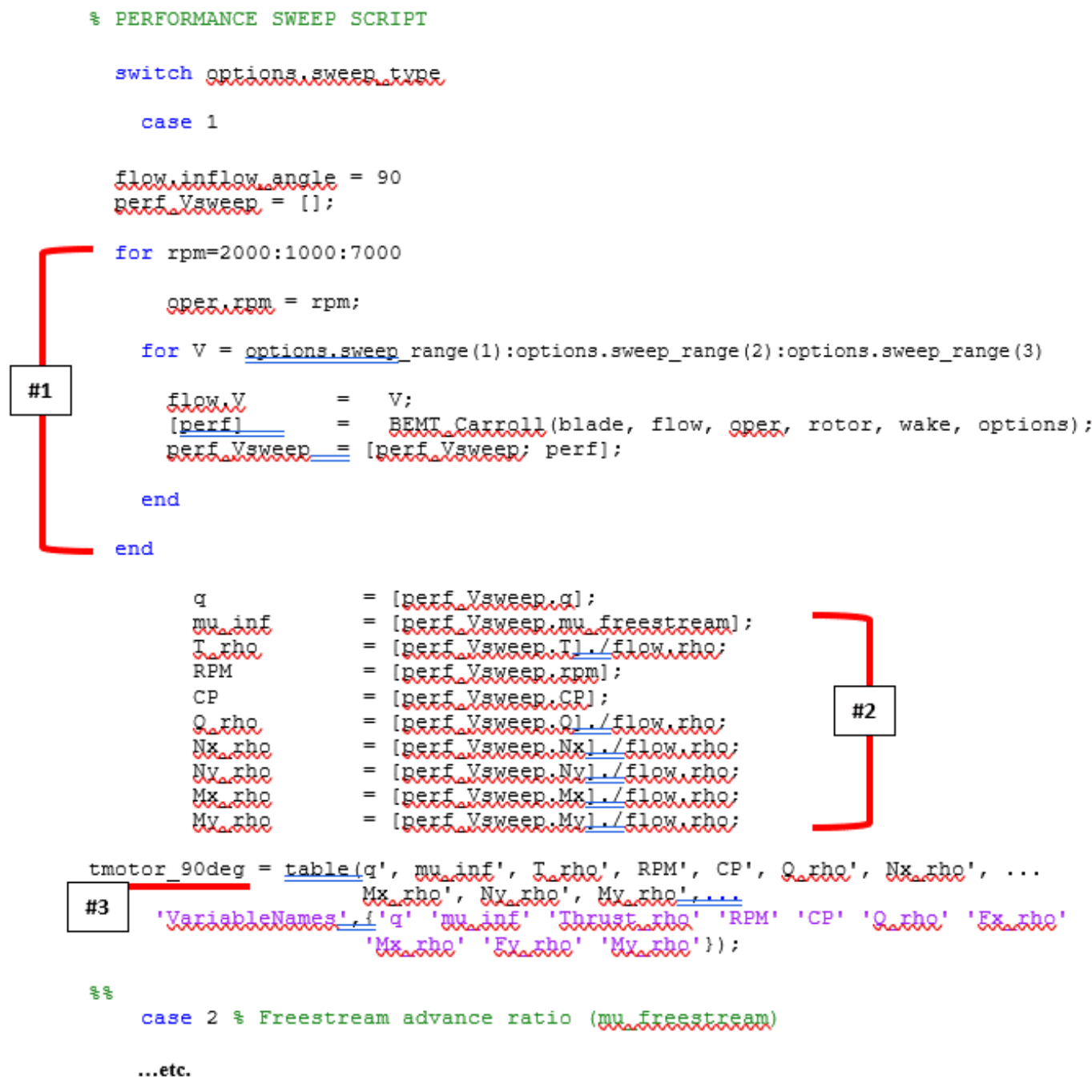

Performance sweep code modification to make FMPP rotor performance lookup tables. 
The workspace variable tmotor_90deg can be saved into the current document folder with a file name usable by the FMPP code by entering the following into the command window:

save('90_tmotor','tmotor_90deg')

To update the pitch angle for the next set of rotor performance lookup table data, change the variable flow.inflow_angle $=90$ to the next pitch angle and update the workspace file name tmotor_90deg to the next pitch value.

Update rotor names as required. Save files into folder with the same rotor name as the files. Save folder in rotor folder in FMPP folder, as shown in Fig. ???

Note:

- It is important to ensure that there is sufficient RPM data for rotor file and sufficient angle of attack cases for smooth and connected results. 


\section{B.1.6 Analysis Flags}

There are four analysis flags that can be turned on (1) or off (0). These flags are in the main FMPP program as shown in Fig. ??

The following results will not be calculated if the analysis flag is turned off.

- Moment trim if zero, rotor thrusts will not be adjusted for moment trim

- Rotor interference if zero, interference due to the surrounding rotors will not be added to the inflow model

- Body interference if zero, interference due to the body and freestream interactions will not be added to the inflow model

- Turn body forces on or off if zero, induced drag and lift of the central body will not be calculated

Turning the analysis types off will improve processing time.

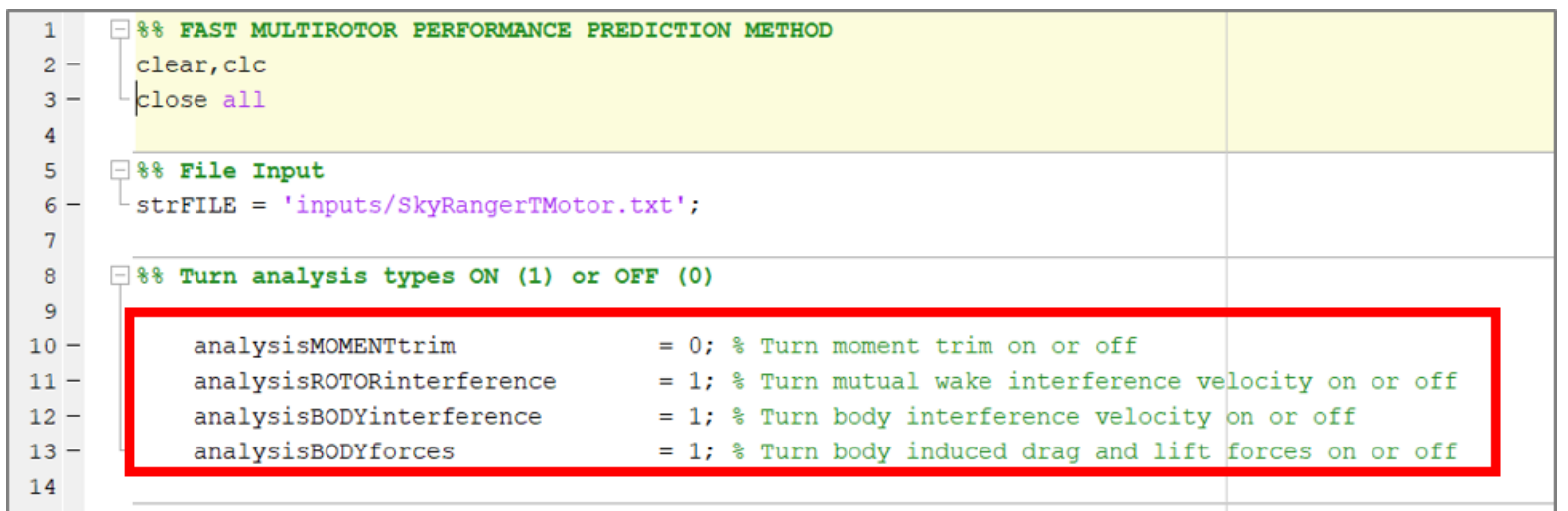

Analysis type selection in FMPP program. 


\section{B.1.7 Adding a Mass Offset}

A point mass can be added to the analysis by entering the mass in kilograms and position in meters into the following section of the FMPP program in Fig. ??. The centre of gravity will also be updated. This section was created as an option to add a non-symmetric mass distribution.

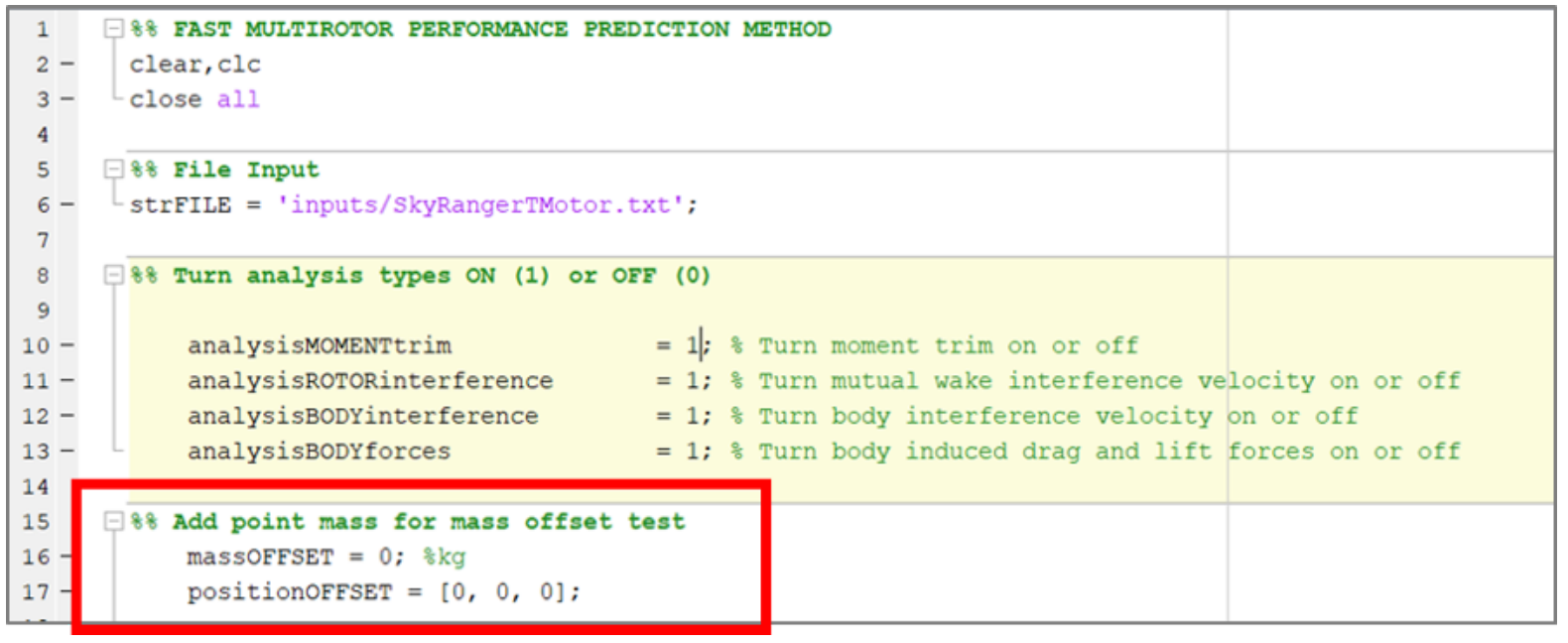

Adding a mass offset. 


\section{B.2 Running the FMPP Program}

Once the file setup and rotor file setup are complete, run the Main_FMPP.m file by selecting the Run icon shown in Fig. ??

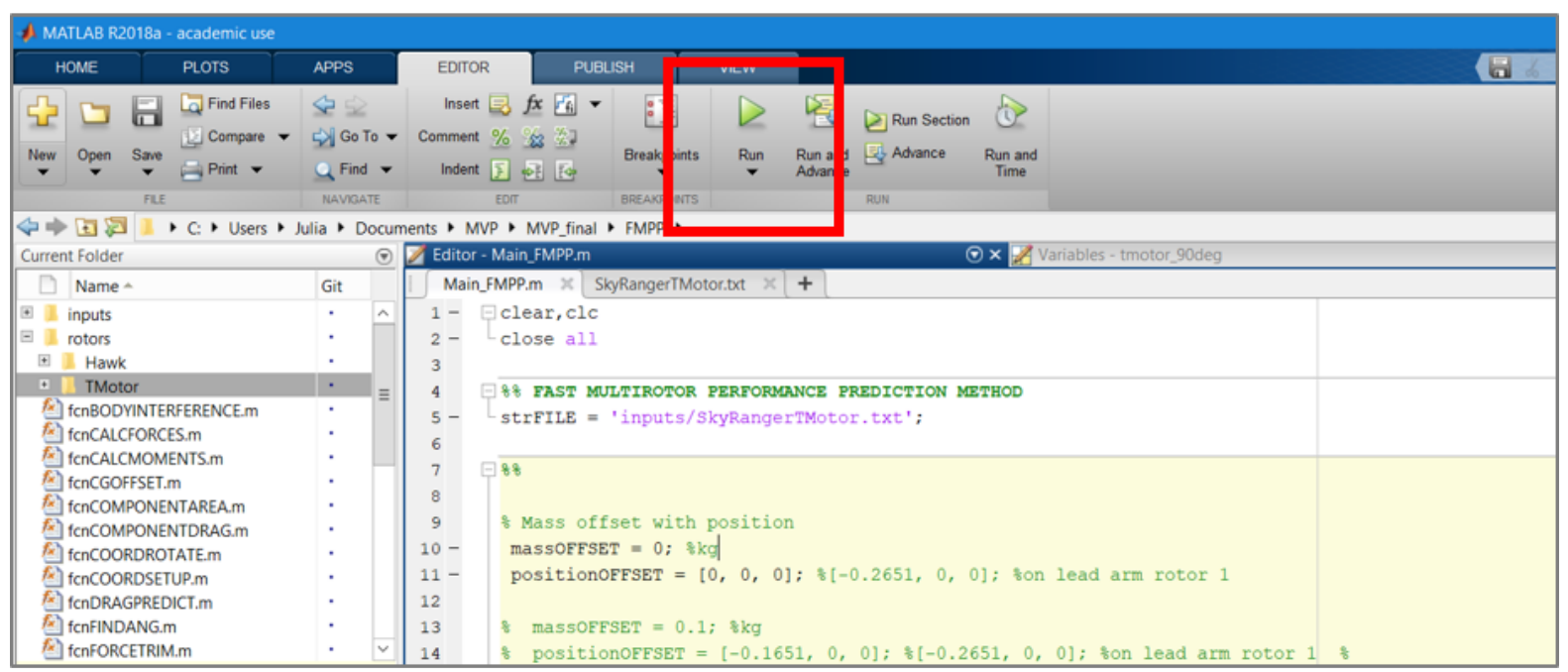

Running FMPP program.

In the command window, updates to the current velocity will show incrementally until the program finishes. 


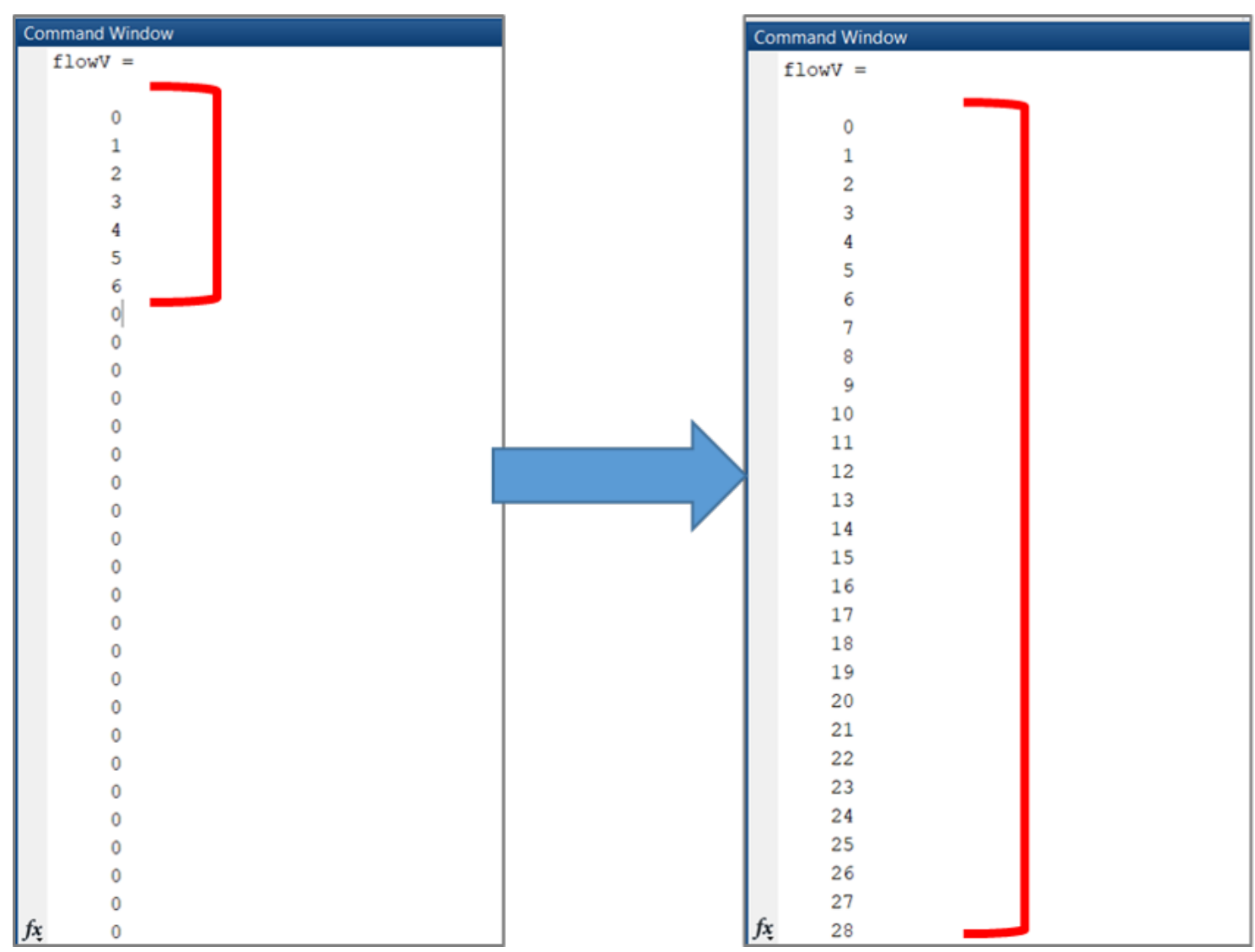

FMPP program command window during run. 


\section{B.3 Outputs}

This section describes the output variables provided by the FMPP code. The variable types are categorized by the following:

- rotor forces and moments

- drag and body lift forces

- moments of components

- interference velocities

- power and pitch output variables

In addition, array dimensions are provided describing the variable structure as it is output in the MATLAB workspace.

$\mathrm{m}=$ velocity increments set by 'seqV' vector input $\mathrm{n}=$ number of rotors 


\section{B.3.1 Rotor Forces and Moments}
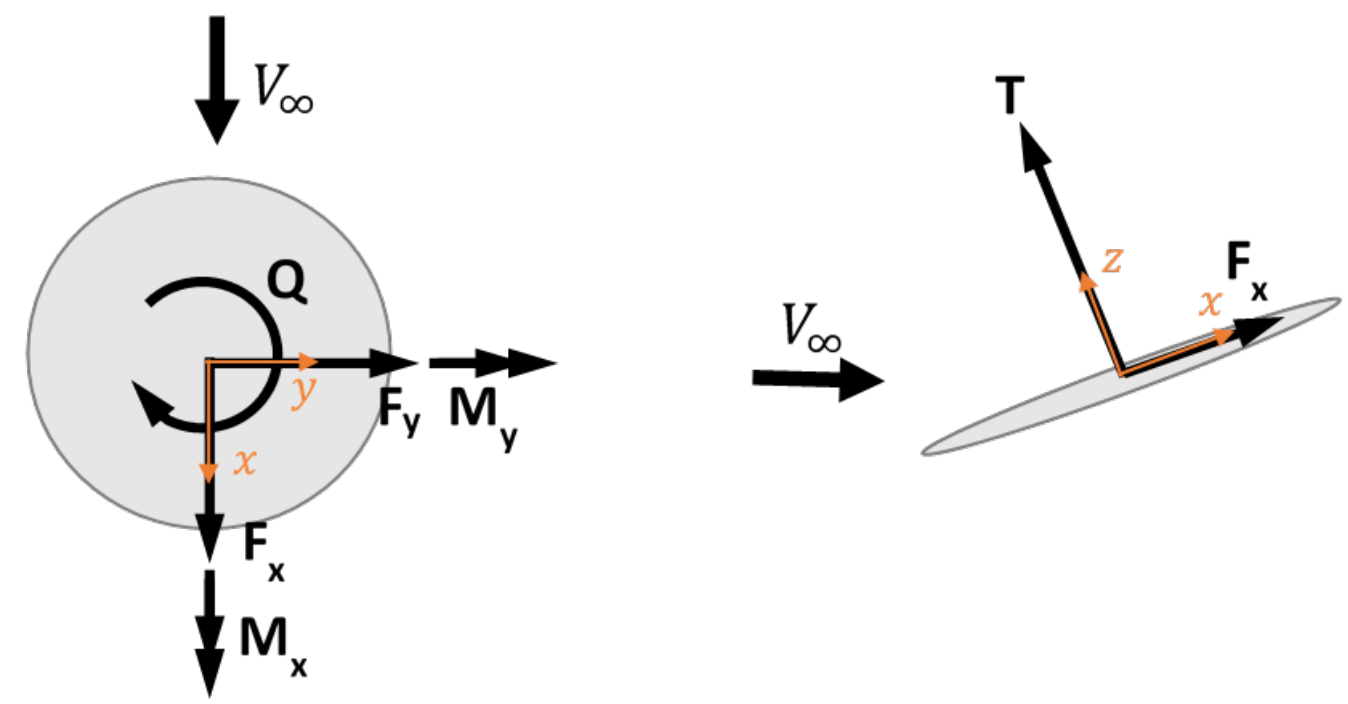

Rotor force and moment convention.

\begin{tabular}{|c|c|c|c|c|}
\hline Symbol & Unit & Variable & Variable Name & $\begin{array}{l}\text { Array } \\
\text { Dimension }\end{array}$ \\
\hline $\mathrm{T}$ & $\mathrm{N}$ & Rotor Thrust & rotorTHRUST & $m \times 1 \times n$ \\
\hline Fx & $\mathrm{N}$ & Longitudinal Hub Drag & rotorFx & $m \times 1 \times n$ \\
\hline Fy & $\mathrm{N}$ & Lateral Hub Drag & rotorFy & $m \times 1 \times n$ \\
\hline Mx & $\mathrm{Nm}$ & Rotor Rolling Moment & rotorMy & $m \times 1 \times n$ \\
\hline My & $\mathrm{Nm}$ & Rotor Pitching Moment & rotorFx & $m \times 1 \times n$ \\
\hline \multirow[t]{4}{*}{ Q } & $\mathrm{Nm}$ & Rotor Torque & rotorQ & $m \times 1 \times n$ \\
\hline & - & Rotor Power Coefficient & rotorCP & $m \times 1 \times n$ \\
\hline & W & Rotor Power & rotorPOWER & $m \times 1 \times n$ \\
\hline & RPM & Rotor Speed & rotorRPM & $m \times 1 \times n$ \\
\hline
\end{tabular}

Output rotor force and moment variables. 


\section{B.3.2 Drag and Body Lift Forces [N]}

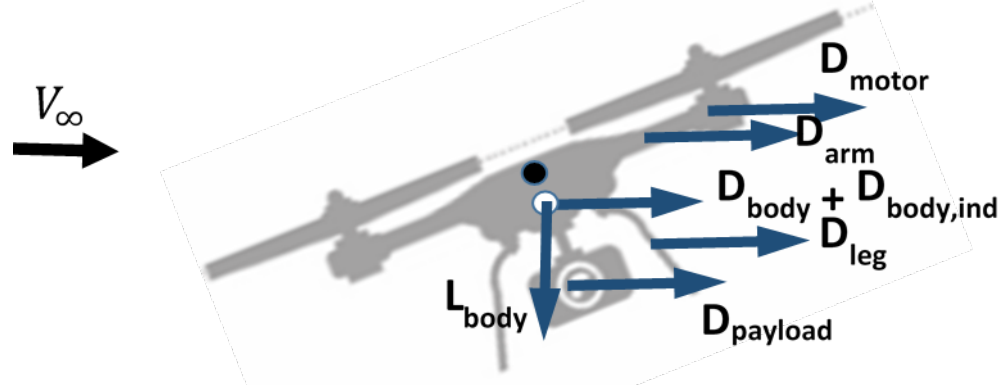

- origin $\bigcirc$ centre of gravity

Example of vehicle drag forces, induced drag and lift of the central body.

\begin{tabular}{|c|c|c|c|}
\hline Symbol & Variable & Variable Name & $\begin{array}{l}\text { Array } \\
\text { Dimension }\end{array}$ \\
\hline$D_{\text {motor }}$ & Drag of the motors & dragMOTOR & $m \times 3 \times n$ \\
\hline$D_{\text {arm }}$ & Drag of the arms & dragARM & $m \times 3 \times n$ \\
\hline$D_{\text {leg }}$ & Drag of the legs & dragLEG & $m \times 3 \times 4$ \\
\hline$D_{\text {body }}$ & Parasitic drag of central body & dragBODY & $\mathrm{m} \times 3 \times 1$ \\
\hline$D_{\text {body,ind }}$ & Induced drag of central body & dragBODYinduced & $\mathrm{m} \times 3 \times 1$ \\
\hline$L_{\text {body }}$ & Lift of central body & liftBODY & $m \times 3 \times 1$ \\
\hline$D_{\text {payload }}$ & Drag of payload & dragPAYLOAD & $m \times 3 \times 1$ \\
\hline
\end{tabular}

Output drag and lift variables. 


\section{B.3.3 Moments of Components [Nm]}

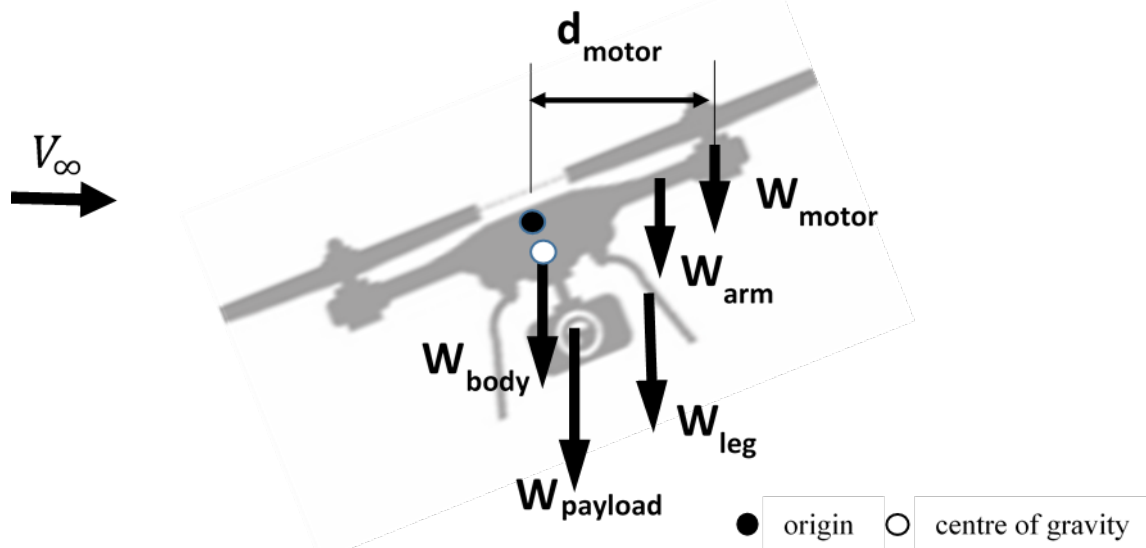

Example of vehicle weight forces and the moment arm of the motor weight.

\begin{tabular}{|c|c|c|c|}
\hline Symbol & Variable & Variable Name & $\begin{array}{l}\text { Array } \\
\text { Dimension }\end{array}$ \\
\hline & Moment due to the rotor thrusts & momentTHRUST & $\mathrm{mx} 3 \mathrm{xn}$ \\
\hline & Moment due to the long. hub drag & momentROTORFx & $\mathrm{mx} 3 \mathrm{xn}$ \\
\hline & " " lateral hub drag & momentROTORFy & $\mathrm{mx} 3 \mathrm{xn}$ \\
\hline & " " " rotor rolling moment & momentROTORMx & $\operatorname{mx} 3 x n$ \\
\hline & " " " rotor pitching moment & momentROTORMy & $\mathrm{mx} 3 \mathrm{xn}$ \\
\hline & " " rotor torque & momentROTORQ & $\mathrm{mx} 3 \mathrm{xn}$ \\
\hline & "” " weight of the motors & momentWEIGHTMOTOR & $\operatorname{mx} 3 x n$ \\
\hline & " " " drag of the motors & momentDRAGMOTOR & $\mathrm{mx} 3 \mathrm{xn}$ \\
\hline & " " " weight of the arms & momentWEIGHTARM & $\operatorname{mx} 3 x n$ \\
\hline & "” " drag of the arms & momentDRAGARM & $\mathrm{mx} 3 \mathrm{xn}$ \\
\hline & " " "weight of the legs & momentWEIGHTLEG & $\mathrm{mx} 3 \mathrm{x} 4$ \\
\hline & "” " drag of the legs & momentDRAGLEG & $\mathrm{mx} 3 \mathrm{x} 4$ \\
\hline & "” " weight of the body & momentWEIGHTBODY & $\mathrm{mx} 3 \mathrm{x} 1$ \\
\hline & "” " parasitic drag of central body & momentDRAGBODY & $\mathrm{mx} 3 \mathrm{x} 1$ \\
\hline & "” " induced drag of central body & momentDRAGBODYinduced & $\mathrm{mx} 3 \mathrm{x} 1$ \\
\hline & " " " lift of central body & momentLIFTBODY & $\mathrm{mx} 3 \mathrm{x} 1$ \\
\hline & “" " weight of payload & momentWEIGHTPAYLOAD & $\mathrm{mx} 3 \mathrm{x} 1$ \\
\hline & “" " drag of payload & momentDRAGPAYLOAD & $\mathrm{mx} 3 \mathrm{x} 1$ \\
\hline & “" " weight of mass offset & momentWEIGHTOFFSET & $\mathrm{mx} 3 \mathrm{x} 1$ \\
\hline & Total moments of vehicle & momentTOTAL & $\operatorname{mx} 3 \times 1$ \\
\hline
\end{tabular}

Output moments of vehicle forces and moments. 


\section{B.3.4 Interference Velocity}

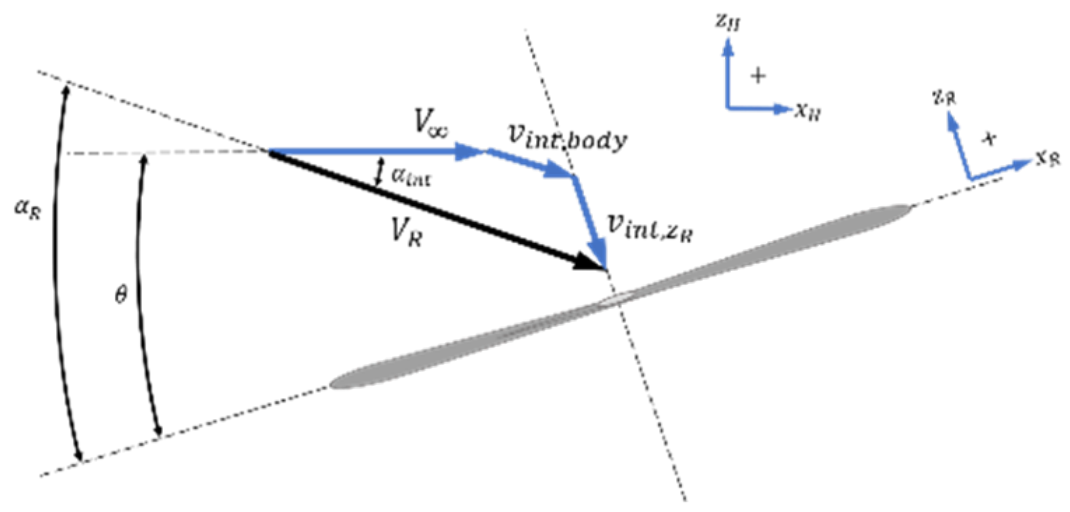

Vector addition of rotor interference, $v_{i n t}$, and body interference, $v_{i n t, b o d y}$, and freestream velocities.

\begin{tabular}{|c|c|c|c|}
\hline Symbol & Variable & Variable Name & $\begin{array}{l}\text { Array } \\
\text { Dimension }\end{array}$ \\
\hline $\mathrm{V}_{\infty}$ & Freestream velocity & flowV & $\mathrm{mxl}$ \\
\hline \multirow[t]{3}{*}{$V_{\text {int }, z R}$} & Mutual interference velocity & vi_int & $m \times 3 x n$ \\
\hline & Self induced velocity & vi_self & $\mathrm{mx} 3 \mathrm{xn}$ \\
\hline & Mutual interference component & wi & $\mathrm{mx} 3 \mathrm{xn}$ \\
\hline$V_{\text {int,body }}$ & Fuselage interference & vi_body & $\mathrm{mx} 3 \mathrm{xn}$ \\
\hline$\alpha_{\mathrm{R}}$ & Rotor resultant inflow angle & rotorANGinflow & $\operatorname{mx} 1 x n$ \\
\hline$V_{R}$ & Rotor resultant & rotorVELinflow & $\operatorname{mxlxn}$ \\
\hline
\end{tabular}

Output interference velocities. 


\section{B.3.5 Power and Pitch Output Variables}

\begin{tabular}{|l|l|l|l|l}
\hline \multirow{2}{*}{ Symbol } & Unit & Variable & Variable Name & $\begin{array}{l}\text { Array } \\
\text { Dimension }\end{array}$ \\
\hline \multicolumn{5}{|l|}{} \\
& W & Rotor power & powerROTOR & mx1xn \\
\hline & W & Parasitic power & powerPARASITIC & $\mathrm{mx1}$ \\
\hline & W & Vehicle power & powerVEHICLE & $\mathrm{mx} 1$ \\
\hline & degree & Vehicle pitch & pitchVEHICLEdeg & $\mathrm{mx} 1$ \\
\hline
\end{tabular}

Power and pitch output variables. 


\title{
Appendix C
}

ROTOR PERFORMANCE

PREDICTION DATA

\author{
C.1 3DR Solo Rotor Performance Tables
}


GRCSP 10x4.5 performance data table at $\theta=0$ deg.

\begin{tabular}{|c|c|c|c|c|c|c|c|c|c|c|}
\hline $\mathrm{q}, \mathrm{Pa}$ & T_rho, N & $\mathrm{RPM}$ & $\mathrm{J}_{\infty}$ & $\mathrm{CP}$ & Q_rho, Nm & Fx_rho, N & Fy_rho, N & Mx_rho, Nm & My_rho, Nm & $\theta, \operatorname{deg}$ \\
\hline 0.0 & 3.031 & 5000 & 0.000 & 0.002 & 0.070 & 0.000 & 0.000 & 0.000 & 0.000 & 0 \\
\hline 0.6 & 3.013 & 5000 & 0.047 & 0.002 & 0.070 & -0.032 & -0.003 & -0.004 & 0.005 & 0 \\
\hline 2.5 & 3.000 & 5000 & 0.094 & 0.002 & 0.071 & -0.072 & -0.015 & -0.013 & 0.008 & 0 \\
\hline 5.5 & 2.912 & 5000 & 0.142 & 0.002 & 0.072 & -0.104 & -0.025 & -0.014 & 0.011 & 0 \\
\hline 9.8 & 2.891 & 5000 & 0.189 & 0.002 & 0.074 & -0.137 & -0.036 & -0.018 & 0.018 & 0 \\
\hline 15.3 & 2.887 & 5000 & 0.236 & 0.002 & 0.078 & -0.188 & -0.047 & -0.027 & 0.017 & 0 \\
\hline 22.1 & 2.841 & 5000 & 0.283 & 0.002 & 0.079 & -0.228 & -0.059 & -0.031 & 0.017 & 0 \\
\hline 30.0 & 2.848 & 5000 & 0.331 & 0.002 & 0.080 & -0.264 & -0.069 & -0.037 & 0.015 & 0 \\
\hline 39.2 & 2.877 & 5000 & 0.378 & 0.002 & 0.083 & -0.309 & -0.069 & -0.044 & 0.017 & 0 \\
\hline 49.6 & 2.825 & 5000 & 0.425 & 0.002 & 0.084 & -0.348 & -0.070 & -0.042 & 0.014 & 0 \\
\hline 61.3 & 2.844 & 5000 & 0.472 & 0.002 & 0.085 & -0.390 & -0.073 & -0.049 & 0.010 & 0 \\
\hline 0.0 & 4.380 & 6000 & 0.000 & 0.002 & 0.100 & 0.000 & 0.000 & 0.000 & 0.000 & 0 \\
\hline 0.6 & 4.404 & 6000 & 0.039 & 0.002 & 0.101 & -0.041 & -0.007 & -0.008 & 0.005 & 0 \\
\hline 2.5 & 4.309 & 6000 & 0.079 & 0.002 & 0.100 & -0.082 & -0.023 & -0.015 & 0.007 & 0 \\
\hline 5.5 & 4.292 & 6000 & 0.118 & 0.002 & 0.103 & -0.128 & -0.030 & -0.023 & 0.014 & 0 \\
\hline 9.8 & 4.252 & 6000 & 0.157 & 0.002 & 0.105 & -0.165 & -0.039 & -0.021 & 0.019 & 0 \\
\hline 15.3 & 4.179 & 6000 & 0.197 & 0.002 & 0.107 & -0.206 & -0.055 & -0.027 & 0.027 & 0 \\
\hline 22.1 & 4.127 & 6000 & 0.236 & 0.002 & 0.111 & -0.273 & -0.074 & -0.042 & 0.024 & 0 \\
\hline 30.0 & 4.089 & 6000 & 0.276 & 0.002 & 0.113 & -0.318 & -0.085 & $\begin{array}{c}-0.043 \\
\end{array}$ & 0.026 & 0 \\
\hline 39.2 & 4.087 & 6000 & 0.315 & 0.002 & 0.114 & -0.366 & $\begin{array}{l}-0.099 \\
\end{array}$ & -0.050 & 0.023 & 0 \\
\hline 49.6 & 4.135 & 6000 & 0.354 & 0.002 & 0.117 & -0.410 & -0.105 & -0.057 & 0.024 & 0 \\
\hline 61.3 & 4.164 & 6000 & 0.394 & 0.002 & 0.119 & -0.464 & -0.102 & -0.066 & 0.026 & 0 \\
\hline 0.0 & 5.989 & 7000 & 0.000 & 0.002 & 0.135 & 0.000 & 0.000 & 0.000 & 0.000 & 0 \\
\hline 0.6 & 6.090 & 7000 & 0.034 & 0.002 & 0.136 & -0.047 & -0.009 & -0.009 & 0.001 & 0 \\
\hline 2.5 & 5.889 & 7000 & 0.067 & 0.002 & 0.135 & -0.101 & -0.014 & -0.018 & 0.014 & 0 \\
\hline 5.5 & 5.927 & 7000 & 0.101 & 0.002 & 0.138 & -0.155 & -0.033 & -0.027 & 0.018 & 0 \\
\hline 9.8 & 5.877 & 7000 & 0.135 & 0.002 & 0.141 & -0.193 & -0.043 & -0.031 & 0.031 & 0 \\
\hline 15.3 & 5.759 & 7000 & 0.169 & 0.002 & 0.143 & -0.238 & -0.072 & -0.026 & 0.034 & 0 \\
\hline 22.1 & 5.716 & 7000 & 0.202 & 0.002 & 0.145 & -0.288 & -0.083 & -0.039 & 0.039 & 0 \\
\hline 30.0 & 5.691 & 7000 & 0.236 & 0.002 & 0.151 & -0.366 & -0.097 & -0.054 & 0.040 & 0 \\
\hline 39.2 & 5.574 & 7000 & 0.270 & 0.002 & 0.152 & -0.423 & -0.117 & -0.058 & 0.037 & 0 \\
\hline 49.6 & 5.623 & 7000 & 0.304 & 0.002 & 0.155 & -0.478 & -0.125 & -0.066 & 0.039 & 0 \\
\hline 61.3 & 5.613 & 7000 & 0.337 & 0.002 & 0.157 & -0.529 & -0.141 & -0.074 & 0.034 & 0 \\
\hline
\end{tabular}


GRCSP $10 \mathrm{x} 4.5$ performance data table at $\theta=5 \mathrm{deg}$.

\begin{tabular}{|c|c|c|c|c|c|c|c|c|c|c|}
\hline $\mathrm{q}, \mathrm{Pa}$ & T_rho, N & $\mathrm{RPM}$ & $\mathrm{J}_{\infty}$ & $\mathrm{CP}$ & Q_rho, Nm & Fx_rho, N & Fy_rho, N & Mx_rho, Nm & My_rho, Nm & $\theta, \operatorname{deg}$ \\
\hline 0.0 & 3.031 & 5000 & 0.000 & 0.002 & 0.070 & 0.000 & 0.000 & 0.000 & 0.000 & 5 \\
\hline 0.6 & 3.043 & 5000 & 0.047 & 0.002 & 0.070 & -0.037 & -0.008 & -0.006 & 0.002 & 5 \\
\hline 2.5 & 2.990 & 5000 & 0.094 & 0.002 & 0.070 & -0.068 & -0.011 & -0.013 & 0.008 & 5 \\
\hline 5.5 & 2.992 & 5000 & 0.142 & 0.002 & 0.072 & -0.103 & -0.022 & -0.017 & 0.009 & 5 \\
\hline 9.8 & 2.908 & 5000 & 0.189 & 0.002 & 0.073 & -0.137 & -0.029 & -0.016 & 0.014 & 5 \\
\hline 15.3 & 2.894 & 5000 & 0.236 & 0.002 & 0.075 & -0.175 & -0.036 & -0.024 & 0.017 & 5 \\
\hline 22.1 & 2.919 & 5000 & 0.283 & 0.002 & 0.078 & -0.222 & -0.042 & -0.030 & 0.017 & 5 \\
\hline 30.0 & 2.850 & 5000 & 0.331 & 0.002 & 0.078 & -0.261 & -0.052 & -0.036 & 0.017 & 5 \\
\hline 39.2 & 2.843 & 5000 & 0.378 & 0.002 & 0.079 & -0.300 & -0.058 & -0.042 & 0.015 & 5 \\
\hline 49.6 & 2.876 & 5000 & 0.425 & 0.002 & 0.080 & -0.339 & -0.058 & -0.048 & 0.017 & 5 \\
\hline 61.3 & 2.908 & 5000 & 0.472 & 0.002 & 0.081 & -0.380 & -0.056 & -0.054 & 0.015 & 5 \\
\hline 0.0 & 4.380 & 6000 & 0.000 & 0.002 & 0.100 & 0.000 & 0.000 & 0.000 & 0.000 & 5 \\
\hline 0.6 & 4.448 & 6000 & 0.039 & 0.002 & 0.101 & -0.043 & -0.009 & -0.008 & -0.001 & 5 \\
\hline 2.5 & 4.298 & 6000 & 0.079 & 0.002 & 0.099 & -0.081 & -0.015 & -0.015 & 0.008 & 5 \\
\hline 5.5 & 4.298 & 6000 & 0.118 & 0.002 & 0.103 & -0.126 & -0.028 & -0.021 & 0.013 & 5 \\
\hline 9.8 & 4.288 & 6000 & 0.157 & 0.002 & 0.104 & -0.165 & -0.035 & -0.027 & 0.019 & 5 \\
\hline 15.3 & 4.204 & 6000 & 0.197 & 0.002 & 0.106 & -0.204 & -0.052 & -0.024 & 0.022 & 5 \\
\hline 22.1 & 4.179 & 6000 & 0.236 & 0.002 & 0.108 & -0.251 & -0.053 & -0.035 & 0.027 & 5 \\
\hline 30.0 & 4.208 & 6000 & 0.276 & 0.002 & 0.110 & -0.305 & -0.056 & $\begin{array}{c}-0.042 \\
\end{array}$ & 0.027 & 5 \\
\hline 39.2 & 4.205 & 6000 & 0.315 & 0.002 & 0.113 & -0.356 & -0.069 & -0.053 & 0.028 & 5 \\
\hline 49.6 & 4.087 & 6000 & 0.354 & 0.002 & 0.113 & -0.404 & -0.084 & -0.056 & 0.022 & 5 \\
\hline 61.3 & 4.115 & 6000 & 0.394 & 0.002 & 0.114 & -0.451 & -0.085 & -0.064 & 0.024 & 5 \\
\hline 0.0 & 5.989 & 7000 & 0.000 & 0.002 & 0.135 & 0.000 & 0.000 & 0.000 & 0.000 & 5 \\
\hline 0.6 & 6.084 & 7000 & 0.034 & 0.002 & 0.136 & -0.047 & -0.009 & -0.009 & 0.001 & 5 \\
\hline 2.5 & 5.877 & 7000 & 0.067 & 0.002 & 0.135 & -0.101 & -0.013 & -0.018 & 0.014 & 5 \\
\hline 5.5 & 5.909 & 7000 & 0.101 & 0.002 & 0.136 & -0.142 & -0.024 & -0.027 & 0.019 & 5 \\
\hline 9.8 & 5.940 & 7000 & 0.135 & 0.002 & 0.140 & -0.190 & -0.041 & -0.028 & 0.021 & 5 \\
\hline 15.3 & 5.792 & 7000 & 0.169 & 0.002 & 0.141 & -0.235 & -0.052 & -0.031 & 0.028 & 5 \\
\hline 22.1 & 5.751 & 7000 & 0.202 & 0.002 & 0.144 & -0.286 & -0.074 & -0.034 & 0.032 & 5 \\
\hline 30.0 & 5.712 & 7000 & 0.236 & 0.002 & 0.145 & -0.335 & -0.081 & -0.047 & 0.039 & 5 \\
\hline 39.2 & 5.744 & 7000 & 0.270 & 0.002 & 0.148 & -0.395 & -0.081 & -0.055 & 0.039 & 5 \\
\hline 49.6 & 5.729 & 7000 & 0.304 & 0.002 & 0.152 & -0.471 & -0.091 & -0.070 & 0.041 & 5 \\
\hline 61.3 & 5.622 & 7000 & 0.337 & 0.002 & 0.153 & -0.521 & -0.107 & -0.072 & 0.038 & 5 \\
\hline
\end{tabular}


GRCSP 10x4.5 performance data table at $\theta=10 \mathrm{deg}$.

\begin{tabular}{|c|c|c|c|c|c|c|c|c|c|c|}
\hline $\mathrm{q}, \mathrm{Pa}$ & T_rho, $\mathrm{N}$ & $\mathrm{RPM}$ & $\mathrm{J}_{\infty}$ & $\mathrm{CP}$ & Q_rho, Nm & Fx_rho, N & Fy_rho, N & Mx_rho, Nm & My_rho, Nm & $\theta$, deg \\
\hline 0.0 & 3.031 & 5000 & 0.000 & 0.002 & 0.070 & 0.000 & 0.000 & 0.000 & 0.000 & 10 \\
\hline 0.6 & 3.038 & 5000 & 0.047 & 0.002 & 0.070 & -0.037 & -0.008 & -0.006 & 0.002 & 10 \\
\hline 2.5 & 2.996 & 5000 & 0.094 & 0.002 & 0.070 & -0.067 & -0.011 & -0.013 & 0.006 & 10 \\
\hline 5.5 & 2.991 & 5000 & 0.142 & 0.002 & 0.072 & -0.098 & -0.018 & -0.015 & 0.008 & 10 \\
\hline 9.8 & 2.956 & 5000 & 0.189 & 0.002 & 0.072 & -0.138 & -0.025 & -0.023 & 0.011 & 10 \\
\hline 15.3 & 2.931 & 5000 & 0.236 & 0.002 & 0.073 & -0.171 & -0.031 & -0.024 & 0.010 & 10 \\
\hline 22.1 & 2.919 & 5000 & 0.283 & 0.002 & 0.074 & -0.205 & -0.037 & -0.028 & 0.013 & 10 \\
\hline 30.0 & 2.910 & 5000 & 0.331 & 0.002 & 0.076 & -0.246 & -0.034 & -0.036 & 0.018 & 10 \\
\hline 39.2 & 2.932 & 5000 & 0.378 & 0.002 & 0.076 & -0.283 & -0.033 & -0.042 & 0.018 & 10 \\
\hline 49.6 & 2.934 & 5000 & 0.425 & 0.002 & 0.078 & -0.323 & -0.035 & -0.047 & 0.015 & 10 \\
\hline 61.3 & 2.977 & 5000 & 0.472 & 0.002 & 0.078 & -0.358 & -0.034 & -0.051 & 0.013 & 10 \\
\hline 0.0 & 4.380 & 6000 & 0.000 & 0.002 & 0.100 & 0.000 & 0.000 & 0.000 & 0.000 & 10 \\
\hline 0.6 & 4.443 & 6000 & 0.039 & 0.002 & 0.100 & -0.047 & -0.013 & -0.008 & 0.000 & 10 \\
\hline 2.5 & 4.287 & 6000 & 0.079 & 0.002 & 0.099 & -0.080 & -0.014 & -0.015 & 0.008 & 10 \\
\hline 5.5 & 4.349 & 6000 & 0.118 & 0.002 & 0.102 & -0.121 & -0.018 & -0.020 & 0.014 & 10 \\
\hline 9.8 & 4.326 & 6000 & 0.157 & 0.002 & 0.104 & -0.161 & -0.033 & -0.025 & 0.012 & 10 \\
\hline 15.3 & 4.276 & 6000 & 0.197 & 0.002 & 0.104 & -0.205 & -0.038 & -0.034 & 0.018 & 10 \\
\hline 22.1 & 4.236 & 6000 & 0.236 & 0.002 & 0.105 & -0.246 & -0.045 & -0.035 & 0.017 & 10 \\
\hline 30.0 & 4.213 & 6000 & 0.276 & 0.002 & 0.107 & -0.286 & -0.054 & -0.038 & 0.021 & 10 \\
\hline 39.2 & 4.192 & 6000 & 0.315 & 0.002 & 0.108 & -0.336 & -0.050 & -0.048 & 0.027 & 10 \\
\hline 49.6 & 4.218 & 6000 & 0.354 & 0.002 & 0.109 & -0.380 & -0.048 & -0.056 & 0.028 & 10 \\
\hline 61.3 & 4.244 & 6000 & 0.394 & 0.002 & 0.110 & -0.423 & -0.049 & -0.063 & 0.029 & 10 \\
\hline 0.0 & 5.989 & 7000 & 0.000 & 0.002 & 0.135 & 0.000 & 0.000 & 0.000 & 0.000 & 10 \\
\hline 0.6 & 6.077 & 7000 & 0.034 & 0.002 & 0.136 & -0.047 & -0.009 & -0.009 & 0.001 & 10 \\
\hline 2.5 & 5.932 & 7000 & 0.067 & 0.002 & 0.135 & -0.089 & -0.022 & -0.012 & 0.009 & 10 \\
\hline 5.5 & 5.924 & 7000 & 0.101 & 0.002 & 0.136 & -0.140 & -0.023 & -0.027 & 0.015 & 10 \\
\hline 9.8 & 5.951 & 7000 & 0.135 & 0.002 & 0.138 & -0.188 & -0.032 & -0.031 & 0.019 & 10 \\
\hline 15.3 & 5.838 & 7000 & 0.169 & 0.002 & 0.140 & -0.237 & -0.045 & -0.040 & 0.028 & 10 \\
\hline 22.1 & 5.852 & 7000 & 0.202 & 0.002 & 0.141 & -0.287 & -0.054 & -0.048 & 0.026 & 10 \\
\hline 30.0 & 5.794 & 7000 & 0.236 & 0.002 & 0.142 & -0.334 & -0.063 & -0.048 & 0.026 & 10 \\
\hline 39.2 & 5.756 & 7000 & 0.270 & 0.002 & 0.144 & -0.380 & -0.075 & -0.051 & 0.032 & 10 \\
\hline 49.6 & 5.766 & 7000 & 0.304 & 0.002 & 0.146 & -0.438 & -0.071 & -0.059 & 0.035 & 10 \\
\hline 61.3 & 5.750 & 7000 & 0.337 & 0.002 & 0.147 & -0.491 & -0.071 & -0.072 & 0.040 & 10 \\
\hline
\end{tabular}


GRCSP 10x4.5 performance data table at $\theta=20 \mathrm{deg}$.

\begin{tabular}{|c|c|c|c|c|c|c|c|c|c|c|}
\hline $\mathrm{q}, \mathrm{Pa}$ & T_rho, N & $\mathrm{RPM}$ & $\mathrm{J}_{\infty}$ & $\mathrm{CP}$ & Q_rho, Nm & Fx_rho, N & Fy_rho, N & Mx_rho, Nm & My_rho, Nm & $\theta, \operatorname{deq}$ \\
\hline 0.0 & 3.031 & 5000 & 0.000 & 0.002 & 0.070 & 0.000 & 0.000 & 0.000 & 0.000 & 20 \\
\hline 0.6 & 3.062 & 5000 & 0.047 & 0.002 & 0.070 & -0.035 & -0.007 & -0.006 & -0.001 & 20 \\
\hline 2.5 & 3.052 & 5000 & 0.094 & 0.002 & 0.070 & -0.056 & -0.003 & -0.006 & 0.006 & 20 \\
\hline 5.5 & 3.005 & 5000 & 0.142 & 0.002 & 0.070 & -0.092 & -0.011 & -0.014 & 0.005 & 20 \\
\hline 9.8 & 2.980 & 5000 & 0.189 & 0.002 & 0.071 & -0.130 & -0.016 & -0.021 & 0.005 & 20 \\
\hline 15.3 & 3.041 & 5000 & 0.236 & 0.002 & 0.072 & -0.163 & -0.009 & -0.029 & 0.008 & 20 \\
\hline 22.1 & 3.041 & 5000 & 0.283 & 0.002 & 0.072 & -0.196 & -0.010 & -0.035 & 0.007 & 20 \\
\hline 30.0 & 3.067 & 5000 & 0.331 & 0.002 & 0.073 & -0.226 & -0.012 & -0.039 & 0.007 & 20 \\
\hline 39.2 & 3.066 & 5000 & 0.378 & 0.002 & 0.073 & -0.257 & -0.011 & -0.046 & 0.004 & 20 \\
\hline 49.6 & 3.107 & 5000 & 0.425 & 0.002 & 0.073 & -0.281 & -0.004 & -0.049 & 0.003 & 20 \\
\hline 61.3 & 3.125 & 5000 & 0.472 & 0.002 & 0.073 & -0.313 & -0.003 & -0.055 & 0.001 & 20 \\
\hline 0.0 & 4.380 & 6000 & 0.000 & 0.002 & 0.100 & 0.000 & 0.000 & 0.000 & 0.000 & 20 \\
\hline 0.6 & 4.431 & 6000 & 0.039 & 0.002 & 0.100 & -0.042 & -0.010 & -0.007 & 0.000 & 20 \\
\hline 2.5 & 4.334 & 6000 & 0.079 & 0.002 & 0.100 & -0.070 & -0.015 & -0.008 & 0.005 & 20 \\
\hline 5.5 & 4.357 & 6000 & 0.118 & 0.002 & 0.100 & -0.107 & -0.007 & -0.016 & 0.012 & 20 \\
\hline 9.8 & 4.321 & 6000 & 0.157 & 0.002 & 0.100 & -0.152 & -0.016 & -0.024 & 0.011 & 20 \\
\hline 15.3 & 4.365 & 6000 & 0.197 & 0.002 & 0.102 & -0.198 & -0.017 & -0.035 & 0.012 & 20 \\
\hline 22.1 & 4.403 & 6000 & 0.236 & 0.002 & 0.103 & -0.234 & -0.013 & -0.041 & 0.014 & 20 \\
\hline 30.0 & 4.405 & 6000 & 0.276 & 0.002 & 0.104 & -0.273 & -0.014 & -0.049 & 0.013 & 20 \\
\hline 39.2 & 4.409 & 6000 & 0.315 & 0.002 & 0.104 & -0.314 & -0.017 & -0.056 & 0.012 & 20 \\
\hline 49.6 & 4.443 & 6000 & 0.354 & 0.002 & 0.105 & -0.347 & -0.017 & -0.061 & 0.012 & 20 \\
\hline 61.3 & 4.482 & 6000 & 0.394 & 0.002 & 0.105 & -0.381 & -0.017 & -0.066 & 0.005 & 20 \\
\hline 0.0 & 5.989 & 7000 & 0.000 & 0.002 & 0.135 & 0.000 & 0.000 & 0.000 & 0.000 & 20 \\
\hline 0.6 & 6.065 & 7000 & 0.034 & 0.002 & 0.136 & -0.044 & -0.004 & -0.009 & 0.002 & 20 \\
\hline 2.5 & 6.009 & 7000 & 0.067 & 0.002 & 0.135 & -0.088 & -0.021 & -0.014 & 0.004 & 20 \\
\hline 5.5 & 6.038 & 7000 & 0.101 & 0.002 & 0.136 & -0.119 & -0.008 & -0.013 & 0.015 & 20 \\
\hline 9.8 & 5.958 & 7000 & 0.135 & 0.002 & 0.136 & -0.170 & -0.023 & -0.027 & 0.016 & 20 \\
\hline 15.3 & 5.915 & 7000 & 0.169 & 0.002 & 0.136 & -0.220 & -0.024 & -0.035 & 0.017 & 20 \\
\hline 22.1 & 5.979 & 7000 & 0.202 & 0.002 & 0.138 & -0.277 & -0.025 & -0.049 & 0.019 & 20 \\
\hline 30.0 & 6.033 & 7000 & 0.236 & 0.002 & 0.140 & -0.318 & -0.018 & -0.056 & 0.021 & 20 \\
\hline 39.2 & 6.036 & 7000 & 0.270 & 0.002 & 0.140 & -0.363 & -0.020 & -0.065 & 0.021 & 20 \\
\hline 49.6 & 6.042 & 7000 & 0.304 & 0.002 & 0.140 & -0.410 & -0.024 & -0.074 & 0.020 & 20 \\
\hline 61.3 & 6.091 & 7000 & 0.337 & 0.002 & 0.141 & -0.449 & -0.024 & -0.078 & 0.020 & 20 \\
\hline
\end{tabular}


GRCSP 10x4.5 performance data table at $\theta=40 \mathrm{deg}$.

\begin{tabular}{|c|c|c|c|c|c|c|c|c|c|c|}
\hline $\mathrm{q}, \mathrm{Pa}$ & T_rho, N & $\mathrm{RPM}$ & $\mathrm{J}_{\infty}$ & $\mathrm{CP}$ & Q_rho, Nm & Fx_rho, N & Fy_rho, N & Mx_rho, Nm & My_rho, Nm & $\theta, \operatorname{deq}$ \\
\hline 0.0 & 3.031 & 5000 & 0.000 & 0.002 & 0.070 & 0.000 & 0.000 & 0.000 & 0.000 & 40 \\
\hline 0.6 & 3.058 & 5000 & 0.047 & 0.002 & 0.069 & -0.024 & -0.007 & -0.003 & 0.000 & 40 \\
\hline 2.5 & 3.093 & 5000 & 0.094 & 0.002 & 0.070 & -0.052 & 0.000 & -0.008 & 0.001 & 40 \\
\hline 5.5 & 3.124 & 5000 & 0.142 & 0.002 & 0.070 & -0.068 & -0.003 & -0.012 & -0.001 & 40 \\
\hline 9.8 & 3.101 & 5000 & 0.189 & 0.002 & 0.070 & -0.096 & 0.002 & -0.016 & -0.001 & 40 \\
\hline 15.3 & 3.076 & 5000 & 0.236 & 0.002 & 0.070 & -0.119 & -0.004 & -0.023 & -0.005 & 40 \\
\hline 22.1 & 3.158 & 5000 & 0.283 & 0.002 & 0.070 & -0.146 & 0.011 & -0.030 & -0.005 & 40 \\
\hline 30.0 & 3.114 & 5000 & 0.331 & 0.002 & 0.070 & -0.158 & 0.008 & -0.034 & -0.006 & 40 \\
\hline 39.2 & 3.144 & 5000 & 0.378 & 0.002 & 0.071 & -0.182 & 0.004 & -0.042 & -0.014 & 40 \\
\hline 49.6 & 3.135 & 5000 & 0.425 & 0.002 & 0.072 & -0.204 & 0.005 & -0.050 & -0.011 & 40 \\
\hline 61.3 & 3.055 & 5000 & 0.472 & 0.002 & 0.072 & -0.223 & 0.004 & -0.056 & -0.015 & 40 \\
\hline 0.0 & 4.380 & 6000 & 0.000 & 0.002 & 0.100 & 0.000 & 0.000 & 0.000 & 0.000 & 40 \\
\hline 0.6 & 4.433 & 6000 & 0.039 & 0.002 & 0.099 & -0.028 & -0.011 & -0.003 & 0.001 & 40 \\
\hline 2.5 & 4.501 & 6000 & 0.079 & 0.002 & 0.100 & -0.062 & 0.000 & -0.009 & 0.005 & 40 \\
\hline 5.5 & 4.499 & 6000 & 0.118 & 0.002 & 0.100 & -0.091 & 0.000 & -0.019 & 0.004 & 40 \\
\hline 9.8 & 4.550 & 6000 & 0.157 & 0.002 & 0.101 & -0.105 & 0.003 & -0.018 & 0.002 & 40 \\
\hline 15.3 & 4.495 & 6000 & 0.197 & 0.002 & 0.099 & -0.143 & -0.002 & -0.025 & 0.000 & 40 \\
\hline 22.1 & 4.533 & 6000 & 0.236 & 0.002 & 0.101 & -0.168 & 0.004 & -0.032 & -0.001 & 40 \\
\hline 30.0 & 4.621 & 6000 & 0.276 & 0.002 & 0.101 & $\begin{array}{c}-0.202 \\
\end{array}$ & 0.017 & $\begin{array}{c}-0.042 \\
\end{array}$ & -0.006 & 40 \\
\hline 39.2 & 4.587 & 6000 & 0.315 & 0.002 & 0.101 & -0.215 & 0.015 & -0.047 & -0.006 & 40 \\
\hline 49.6 & 4.576 & 6000 & 0.354 & 0.002 & 0.102 & -0.240 & 0.003 & -0.053 & -0.016 & 40 \\
\hline 61.3 & 4.660 & 6000 & 0.394 & 0.002 & 0.104 & -0.268 & 0.009 & -0.064 & -0.018 & 40 \\
\hline 0.0 & 5.989 & 7000 & 0.000 & 0.002 & 0.135 & 0.000 & 0.000 & 0.000 & 0.000 & 40 \\
\hline 0.6 & 6.075 & 7000 & 0.034 & 0.002 & 0.135 & -0.038 & -0.009 & -0.003 & 0.003 & 40 \\
\hline 2.5 & 6.062 & 7000 & 0.067 & 0.002 & 0.134 & -0.079 & -0.016 & -0.014 & -0.002 & 40 \\
\hline 5.5 & 6.199 & 7000 & 0.101 & 0.002 & 0.134 & -0.107 & -0.001 & -0.022 & 0.009 & 40 \\
\hline 9.8 & 6.232 & 7000 & 0.135 & 0.002 & 0.135 & -0.125 & -0.006 & -0.022 & 0.003 & 40 \\
\hline 15.3 & 6.230 & 7000 & 0.169 & 0.002 & 0.136 & -0.156 & 0.003 & -0.028 & 0.004 & 40 \\
\hline 22.1 & 6.172 & 7000 & 0.202 & 0.002 & 0.134 & -0.199 & -0.002 & -0.036 & 0.001 & 40 \\
\hline 30.0 & 6.243 & 7000 & 0.236 & 0.002 & 0.136 & -0.229 & 0.007 & -0.044 & 0.001 & 40 \\
\hline 39.2 & 6.386 & 7000 & 0.270 & 0.002 & 0.137 & -0.268 & 0.027 & -0.056 & -0.005 & 40 \\
\hline 49.6 & 6.368 & 7000 & 0.304 & 0.002 & 0.137 & -0.281 & 0.023 & -0.060 & -0.005 & 40 \\
\hline 61.3 & 6.305 & 7000 & 0.337 & 0.002 & 0.137 & -0.311 & 0.013 & -0.068 & -0.008 & 40 \\
\hline
\end{tabular}


GRCSP 10x4.5 performance data table at $\theta=90 \mathrm{deg}$.

\begin{tabular}{|c|c|c|c|c|c|c|c|c|c|c|}
\hline $\mathrm{q}, \mathrm{Pa}$ & T_rho, N & $\mathrm{RPM}$ & $\mathrm{J}_{\infty}$ & $\mathrm{CP}$ & Q_rho, Nm & Fx_rho, N & Fy_rho, N & Mx_rho, Nm & My_rho, Nm & $\theta, \operatorname{deq}$ \\
\hline 0.0 & 3.031 & 5000 & 0.000 & 0.002 & 0.070 & 0.000 & 0.000 & 0.000 & 0.000 & 90 \\
\hline 0.6 & 3.096 & 5000 & 0.047 & 0.002 & 0.068 & 0.000 & 0.000 & 0.000 & 0.000 & 90 \\
\hline 2.5 & 3.018 & 5000 & 0.094 & 0.002 & 0.068 & 0.000 & 0.000 & 0.000 & 0.000 & 90 \\
\hline 5.5 & 3.154 & 5000 & 0.142 & 0.002 & 0.070 & 0.000 & 0.000 & 0.000 & 0.000 & 90 \\
\hline 9.8 & 3.141 & 5000 & 0.189 & 0.002 & 0.069 & 0.000 & 0.000 & 0.000 & 0.000 & 90 \\
\hline 15.3 & 3.186 & 5000 & 0.236 & 0.002 & 0.072 & 0.000 & 0.000 & 0.000 & 0.000 & 90 \\
\hline 22.1 & 3.084 & 5000 & 0.283 & 0.002 & 0.072 & 0.000 & 0.000 & 0.000 & 0.000 & 90 \\
\hline 30.0 & 3.025 & 5000 & 0.331 & 0.002 & 0.073 & 0.000 & 0.000 & 0.000 & 0.000 & 90 \\
\hline 39.2 & 2.772 & 5000 & 0.378 & 0.002 & 0.070 & 0.000 & 0.000 & 0.000 & 0.000 & 90 \\
\hline 49.6 & 2.575 & 5000 & 0.425 & 0.002 & 0.068 & 0.000 & 0.000 & 0.000 & 0.000 & 90 \\
\hline 61.3 & 2.384 & 5000 & 0.472 & 0.002 & 0.066 & 0.000 & 0.000 & 0.000 & 0.000 & 90 \\
\hline 0.0 & 4.380 & 6000 & 0.000 & 0.002 & 0.100 & 0.000 & 0.000 & 0.000 & 0.000 & 90 \\
\hline 0.6 & 4.496 & 6000 & 0.039 & 0.002 & 0.102 & 0.000 & 0.000 & 0.000 & 0.000 & 90 \\
\hline 2.5 & 4.419 & 6000 & 0.079 & 0.002 & 0.097 & 0.000 & 0.000 & 0.000 & 0.000 & 90 \\
\hline 5.5 & 4.672 & 6000 & 0.118 & 0.002 & 0.100 & 0.000 & 0.000 & 0.000 & 0.000 & 90 \\
\hline 9.8 & 4.703 & 6000 & 0.157 & 0.002 & 0.102 & 0.000 & 0.000 & 0.000 & 0.000 & 90 \\
\hline 15.3 & 4.563 & 6000 & 0.197 & 0.002 & 0.099 & 0.000 & 0.000 & 0.000 & 0.000 & 90 \\
\hline 22.1 & 4.683 & 6000 & 0.236 & 0.002 & 0.103 & 0.000 & 0.000 & 0.000 & 0.000 & 90 \\
\hline 30.0 & 4.600 & 6000 & 0.276 & 0.002 & 0.105 & 0.000 & 0.000 & 0.000 & 0.000 & 90 \\
\hline 39.2 & 4.375 & 6000 & 0.315 & 0.002 & 0.101 & 0.000 & 0.000 & 0.000 & 0.000 & 90 \\
\hline 49.6 & 4.327 & 6000 & 0.354 & 0.002 & 0.103 & 0.000 & 0.000 & 0.000 & 0.000 & 90 \\
\hline 61.3 & 4.147 & 6000 & 0.394 & 0.002 & 0.103 & 0.000 & 0.000 & 0.000 & 0.000 & 90 \\
\hline 0.0 & 5.989 & 7000 & 0.000 & 0.002 & 0.135 & 0.000 & 0.000 & 0.000 & 0.000 & 90 \\
\hline 0.6 & 6.174 & 7000 & 0.034 & 0.002 & 0.138 & 0.000 & 0.000 & 0.000 & 0.000 & 90 \\
\hline 2.5 & 6.094 & 7000 & 0.067 & 0.002 & 0.131 & 0.000 & 0.000 & 0.000 & 0.000 & 90 \\
\hline 5.5 & 6.486 & 7000 & 0.101 & 0.002 & 0.138 & 0.000 & 0.000 & 0.000 & 0.000 & 90 \\
\hline 9.8 & 6.359 & 7000 & 0.135 & 0.002 & 0.135 & 0.000 & 0.000 & 0.000 & 0.000 & 90 \\
\hline 15.3 & 6.441 & 7000 & 0.169 & 0.002 & 0.139 & 0.000 & 0.000 & 0.000 & 0.000 & 90 \\
\hline 22.1 & 6.288 & 7000 & 0.202 & 0.002 & 0.135 & 0.000 & 0.000 & 0.000 & 0.000 & 90 \\
\hline 30.0 & 6.503 & 7000 & 0.236 & 0.002 & 0.140 & 0.000 & 0.000 & 0.000 & 0.000 & 90 \\
\hline 39.2 & 6.459 & 7000 & 0.270 & 0.002 & 0.143 & 0.000 & 0.000 & 0.000 & 0.000 & 90 \\
\hline 49.6 & 6.223 & 7000 & 0.304 & 0.002 & 0.139 & 0.000 & 0.000 & 0.000 & 0.000 & 90 \\
\hline 61.3 & 6.247 & 7000 & 0.337 & 0.002 & 0.144 & 0.000 & 0.000 & 0.000 & 0.000 & 90 \\
\hline
\end{tabular}


GRCSP 10x4.5 performance data table at $\theta=-5$ deg.

\begin{tabular}{|c|c|c|c|c|c|c|c|c|c|c|}
\hline $\mathrm{q}, \mathrm{Pa}$ & T_rho, N & $\mathrm{RPM}$ & $\mathrm{J}_{\infty}$ & $\mathrm{CP}$ & Q_rho, Nm & Fx_rho, N & Fy_rho, N & Mx_rho, Nm & My_rho, Nm & $\theta, \operatorname{deg}$ \\
\hline 0.0 & 3.031 & 5000 & 0.000 & 0.002 & 0.070 & 0.000 & 0.000 & 0.000 & 0.000 & -5 \\
\hline 0.6 & 3.018 & 5000 & 0.047 & 0.002 & 0.070 & -0.032 & -0.004 & -0.004 & 0.005 & -5 \\
\hline 2.5 & 2.977 & 5000 & 0.094 & 0.002 & 0.072 & -0.075 & -0.020 & -0.013 & 0.005 & -5 \\
\hline 5.5 & 2.923 & 5000 & 0.142 & 0.002 & 0.073 & -0.104 & -0.029 & -0.014 & 0.011 & -5 \\
\hline 9.8 & 2.847 & 5000 & 0.189 & 0.002 & 0.075 & -0.147 & -0.041 & -0.023 & 0.016 & -5 \\
\hline 15.3 & 2.843 & 5000 & 0.236 & 0.002 & 0.078 & -0.191 & -0.059 & -0.027 & 0.017 & -5 \\
\hline 22.1 & 2.835 & 5000 & 0.283 & 0.002 & 0.081 & -0.227 & -0.076 & -0.031 & 0.014 & -5 \\
\hline 30.0 & 2.870 & 5000 & 0.331 & 0.002 & 0.083 & -0.270 & -0.081 & -0.038 & 0.015 & -5 \\
\hline 39.2 & 2.829 & 5000 & 0.378 & 0.002 & 0.085 & -0.311 & -0.084 & -0.037 & 0.013 & -5 \\
\hline 49.6 & 2.837 & 5000 & 0.425 & 0.002 & 0.087 & -0.356 & -0.088 & -0.045 & 0.013 & -5 \\
\hline 61.3 & 2.881 & 5000 & 0.472 & 0.003 & 0.090 & -0.407 & -0.085 & -0.050 & 0.015 & -5 \\
\hline 0.0 & 4.380 & 6000 & 0.000 & 0.002 & 0.100 & 0.000 & 0.000 & 0.000 & 0.000 & -5 \\
\hline 0.6 & 4.410 & 6000 & 0.039 & 0.002 & 0.101 & -0.041 & -0.008 & -0.008 & 0.005 & -5 \\
\hline 2.5 & 4.295 & 6000 & 0.079 & 0.002 & 0.101 & -0.086 & -0.018 & -0.015 & 0.010 & -5 \\
\hline 5.5 & 4.212 & 6000 & 0.118 & 0.002 & 0.103 & -0.124 & -0.034 & -0.016 & 0.020 & -5 \\
\hline 9.8 & 4.146 & 6000 & 0.157 & 0.002 & 0.106 & -0.171 & -0.049 & -0.023 & 0.024 & -5 \\
\hline 15.3 & 4.114 & 6000 & 0.197 & 0.002 & 0.110 & -0.232 & -0.069 & -0.035 & 0.025 & -5 \\
\hline 22.1 & 4.102 & 6000 & 0.236 & 0.002 & 0.113 & -0.275 & -0.086 & -0.038 & 0.027 & -5 \\
\hline 30.0 & 4.122 & 6000 & 0.276 & 0.002 & 0.116 & -0.316 & -0.103 & $\begin{array}{c}-0.043 \\
\end{array}$ & 0.026 & -5 \\
\hline 39.2 & 4.112 & 6000 & 0.315 & 0.002 & 0.118 & -0.368 & -0.115 & -0.052 & 0.023 & -5 \\
\hline 49.6 & 4.118 & 6000 & 0.354 & 0.002 & 0.121 & -0.420 & -0.120 & -0.052 & 0.025 & -5 \\
\hline 61.3 & 4.093 & 6000 & 0.394 & 0.002 & 0.124 & -0.468 & -0.124 & -0.055 & 0.021 & -5 \\
\hline 0.0 & 5.989 & 7000 & 0.000 & 0.002 & 0.135 & 0.000 & 0.000 & 0.000 & 0.000 & -5 \\
\hline 0.6 & 6.094 & 7000 & 0.034 & 0.002 & 0.137 & -0.051 & -0.004 & -0.009 & 0.001 & -5 \\
\hline 2.5 & 5.900 & 7000 & 0.067 & 0.002 & 0.135 & -0.101 & -0.015 & -0.018 & 0.014 & -5 \\
\hline 5.5 & 5.909 & 7000 & 0.101 & 0.002 & 0.139 & -0.148 & -0.040 & -0.024 & 0.021 & -5 \\
\hline 9.8 & 5.763 & 7000 & 0.135 & 0.002 & 0.140 & -0.194 & -0.054 & -0.026 & 0.027 & -5 \\
\hline 15.3 & 5.655 & 7000 & 0.169 & 0.002 & 0.145 & -0.250 & -0.074 & -0.034 & 0.037 & -5 \\
\hline 22.1 & 5.626 & 7000 & 0.202 & 0.002 & 0.150 & -0.320 & -0.103 & -0.049 & 0.036 & -5 \\
\hline 30.0 & 5.601 & 7000 & 0.236 & 0.002 & 0.153 & -0.374 & -0.119 & -0.052 & 0.039 & -5 \\
\hline 39.2 & 5.616 & 7000 & 0.270 & 0.002 & 0.157 & -0.421 & -0.140 & -0.057 & 0.037 & -5 \\
\hline 49.6 & 5.588 & 7000 & 0.304 & 0.002 & 0.159 & -0.481 & -0.161 & -0.068 & 0.033 & -5 \\
\hline 61.3 & 5.651 & 7000 & 0.337 & 0.002 & 0.163 & -0.544 & -0.162 & -0.076 & 0.035 & -5 \\
\hline
\end{tabular}


GRCSP 10x4.5 performance data table at $\theta=-15$ deg.

\begin{tabular}{|c|c|c|c|c|c|c|c|c|c|c|}
\hline $\mathrm{q}, \mathrm{Pa}$ & T_rho, $\mathrm{N}$ & $\mathrm{RPM}$ & $\mathrm{J}_{\infty}$ & $\mathrm{CP}$ & Q_rho, Nm & Fx_rho, N & Fy_rho, N & Mx_rho, Nm & My_rho, Nm & $\theta$, deg \\
\hline 0.0 & $\begin{array}{l}.031 \\
\end{array}$ & 5000 & 0.000 & 0.002 & 0.070 & 0.000 & 0.000 & 0.000 & 0.000 & -15 \\
\hline 0.6 & 2.994 & 5000 & 0.047 & 0.002 & 0.071 & $\begin{array}{l}-0.033 \\
\end{array}$ & 0.002 & -0.006 & 0.008 & -15 \\
\hline 2.5 & 2.961 & 5000 & 0.094 & 0.002 & 0.072 & -0.073 & -0.020 & -0.012 & 0.008 & -15 \\
\hline 5.5 & 2.847 & 5000 & 0.142 & 0.002 & 0.074 & -0.107 & -0.035 & -0.017 & 0.017 & -15 \\
\hline 9.8 & 2.833 & 5000 & 0.189 & 0.002 & 0.078 & -0.149 & -0.058 & -0.020 & 0.017 & -15 \\
\hline 15.3 & 2.840 & 5000 & 0.236 & 0.002 & 0.081 & -0.185 & -0.080 & -0.026 & 0.015 & -15 \\
\hline 22.1 & 2.831 & 5000 & 0.283 & 0.002 & 0.085 & -0.225 & -0.098 & -0.027 & 0.013 & -15 \\
\hline 30.0 & 2.858 & 5000 & 0.331 & 0.003 & 0.088 & -0.273 & -0.106 & -0.036 & 0.014 & -15 \\
\hline 39.2 & 2.857 & 5000 & 0.378 & 0.003 & 0.092 & -0.316 & -0.118 & -0.037 & 0.011 & -15 \\
\hline 49.6 & 2.885 & 5000 & 0.425 & 0.003 & 0.096 & -0.369 & -0.119 & -0.045 & 0.011 & -15 \\
\hline 61.3 & 2.878 & 5000 & 0.472 & 0.003 & 0.098 & -0.414 & 0.120 & -0.051 & -0.003 & -15 \\
\hline 0.0 & 4.380 & 6000 & 0.000 & 0.002 & 0.100 & 0.000 & 0.000 & 0.000 & 0.000 & -15 \\
\hline 0.6 & 4.420 & 6000 & 0.039 & 0.002 & 0.102 & -0.040 & -0.002 & -0.008 & 0.005 & -15 \\
\hline 2.5 & 4.314 & 6000 & 0.079 & 0.002 & 0.102 & -0.093 & -0.024 & -0.015 & 0.010 & -15 \\
\hline 5.5 & 4.189 & 6000 & 0.118 & 0.002 & 0.104 & -0.123 & -0.040 & -0.015 & 0.015 & -15 \\
\hline 9.8 & 4.115 & 6000 & 0.157 & 0.002 & 0.107 & -0.171 & -0.059 & -0.027 & 0.026 & -15 \\
\hline 15.3 & 4.094 & 6000 & 0.197 & 0.002 & 0.113 & -0.224 & -0.089 & -0.030 & 0.027 & -15 \\
\hline 22.1 & 4.095 & 6000 & 0.236 & 0.002 & 0.117 & -0.267 & -0.116 & -0.037 & 0.024 & -15 \\
\hline 30.0 & 4.065 & 6000 & 0.276 & 0.002 & 0.121 & -0.316 & -0.140 & -0.038 & 0.021 & -15 \\
\hline 39.2 & 4.085 & 6000 & 0.315 & 0.002 & 0.125 & -0.371 & -0.155 & -0.050 & 0.021 & -15 \\
\hline 49.6 & 4.102 & 6000 & 0.354 & 0.003 & 0.130 & -0.424 & -0.165 & -0.051 & 0.018 & -15 \\
\hline 61.3 & 4.129 & 6000 & 0.394 & 0.003 & 0.135 & -0.483 & -0.169 & -0.057 & 0.018 & -15 \\
\hline 0.0 & 5.989 & 7000 & 0.000 & 0.002 & 0.135 & 0.000 & 0.000 & 0.000 & 0.000 & -15 \\
\hline 0.6 & 6.106 & 7000 & 0.034 & 0.002 & 0.138 & -0.049 & -0.004 & -0.009 & 0.001 & -15 \\
\hline 2.5 & 5.921 & 7000 & 0.067 & 0.002 & 0.137 & -0.104 & -0.025 & -0.017 & 0.014 & -15 \\
\hline 5.5 & 5.768 & 7000 & 0.101 & 0.002 & 0.140 & -0.147 & -0.050 & -0.017 & 0.018 & -15 \\
\hline 9.8 & 5.621 & 7000 & 0.135 & 0.002 & 0.144 & -0.199 & -0.068 & -0.032 & 0.037 & -15 \\
\hline 15.3 & 5.628 & 7000 & 0.169 & 0.002 & 0.150 & -0.262 & -0.102 & -0.039 & 0.037 & -15 \\
\hline 22.1 & 5.598 & 7000 & 0.202 & 0.002 & 0.153 & -0.315 & -0.128 & -0.043 & 0.038 & -15 \\
\hline 30.0 & 5.589 & 7000 & 0.236 & 0.002 & 0.158 & -0.363 & -0.160 & -0.051 & 0.035 & -15 \\
\hline 39.2 & 5.532 & 7000 & 0.270 & 0.002 & 0.163 & -0.420 & -0.189 & -0.050 & 0.030 & -15 \\
\hline 49.6 & 5.567 & 7000 & 0.304 & 0.002 & 0.168 & -0.482 & -0.206 & -0.063 & 0.032 & -15 \\
\hline 61.3 & 5.545 & 7000 & 0.337 & 0.003 & 0.174 & -0.551 & -0.221 & -0.066 & 0.025 & -15 \\
\hline
\end{tabular}


GRCSP 10x4.5 performance data table at $\theta=-90 \mathrm{deg}$.

\begin{tabular}{|c|c|c|c|c|c|c|c|c|c|c|}
\hline $\mathrm{q}, \mathrm{Pa}$ & T_rho, N & $\mathrm{RPM}$ & $\mathrm{J}_{\infty}$ & $\mathrm{CP}$ & Q_rho, Nm & Fx_rho, N & Fy_rho, N & Mx_rho, Nm & My_rho, Nm & $\theta$, deg \\
\hline 0.0 & 3.031 & 5000 & 0.000 & 0.002 & 0.070 & 0.000 & 0.000 & 0.000 & 0.000 & -90 \\
\hline 0.6 & 3.086 & 5000 & 0.047 & 0.002 & 0.072 & 0.000 & 0.000 & 0.000 & 0.000 & -90 \\
\hline 2.5 & 2.851 & 5000 & 0.094 & 0.002 & 0.071 & 0.000 & 0.000 & 0.000 & 0.000 & -90 \\
\hline 5.5 & 2.883 & 5000 & 0.142 & 0.002 & 0.071 & 0.000 & 0.000 & 0.000 & 0.000 & -90 \\
\hline 9.8 & 2.913 & 5000 & 0.189 & 0.002 & 0.076 & 0.000 & 0.000 & 0.000 & 0.000 & -90 \\
\hline 15.3 & 2.799 & 5000 & 0.236 & 0.002 & 0.076 & 0.000 & 0.000 & 0.000 & 0.000 & -90 \\
\hline 22.1 & 2.819 & 5000 & 0.283 & 0.002 & 0.076 & 0.000 & 0.000 & 0.000 & 0.000 & -90 \\
\hline 30.0 & 2.833 & 5000 & 0.331 & 0.002 & 0.079 & 0.000 & 0.000 & 0.000 & 0.000 & -90 \\
\hline 39.2 & 2.847 & 5000 & 0.378 & 0.002 & 0.079 & 0.000 & 0.000 & 0.000 & 0.000 & -90 \\
\hline 49.6 & 2.620 & 5000 & 0.425 & 0.002 & 0.080 & 0.000 & 0.000 & 0.000 & 0.000 & -90 \\
\hline 61.3 & 2.627 & 5000 & 0.472 & 0.002 & 0.080 & 0.000 & 0.000 & 0.000 & 0.000 & -90 \\
\hline 0.0 & 4.380 & 6000 & 0.000 & 0.002 & 0.100 & 0.000 & 0.000 & 0.000 & 0.000 & -90 \\
\hline 0.6 & 4.443 & 6000 & 0.039 & 0.002 & 0.101 & 0.000 & 0.000 & 0.000 & 0.000 & -90 \\
\hline 2.5 & 4.089 & 6000 & 0.079 & 0.002 & 0.101 & 0.000 & 0.000 & 0.000 & 0.000 & -90 \\
\hline 5.5 & 4.129 & 6000 & 0.118 & 0.002 & 0.102 & 0.000 & 0.000 & 0.000 & 0.000 & -90 \\
\hline 9.8 & 4.160 & 6000 & 0.157 & 0.002 & 0.108 & 0.000 & 0.000 & 0.000 & 0.000 & -90 \\
\hline 15.3 & 3.995 & 6000 & 0.197 & 0.002 & 0.108 & 0.000 & 0.000 & 0.000 & 0.000 & -90 \\
\hline 22.1 & 4.021 & 6000 & 0.236 & 0.002 & 0.109 & 0.000 & 0.000 & 0.000 & 0.000 & -90 \\
\hline 30.0 & 4.045 & 6000 & 0.276 & 0.002 & 0.110 & 0.000 & 0.000 & 0.000 & 0.000 & -90 \\
\hline 39.2 & 4.063 & 6000 & 0.315 & 0.002 & 0.110 & 0.000 & 0.000 & 0.000 & 0.000 & -90 \\
\hline 49.6 & 4.078 & 6000 & 0.354 & 0.002 & 0.114 & 0.000 & 0.000 & 0.000 & 0.000 & -90 \\
\hline 61.3 & 4.092 & 6000 & 0.394 & 0.002 & 0.115 & 0.000 & 0.000 & 0.000 & 0.000 & -90 \\
\hline 0.0 & 5.989 & 7000 & 0.000 & 0.002 & 0.135 & 0.000 & 0.000 & 0.000 & 0.000 & -90 \\
\hline 0.6 & 6.056 & 7000 & 0.034 & 0.002 & 0.136 & 0.000 & 0.000 & 0.000 & 0.000 & -90 \\
\hline 2.5 & 5.559 & 7000 & 0.067 & 0.002 & 0.136 & 0.000 & 0.000 & 0.000 & 0.000 & -90 \\
\hline 5.5 & 5.605 & 7000 & 0.101 & 0.002 & 0.137 & 0.000 & 0.000 & 0.000 & 0.000 & -90 \\
\hline 9.8 & 5.644 & 7000 & 0.135 & 0.002 & 0.138 & 0.000 & 0.000 & 0.000 & 0.000 & -90 \\
\hline 15.3 & 5.677 & 7000 & 0.169 & 0.002 & 0.147 & 0.000 & 0.000 & 0.000 & 0.000 & -90 \\
\hline 22.1 & 5.441 & 7000 & 0.202 & 0.002 & 0.147 & 0.000 & 0.000 & 0.000 & 0.000 & -90 \\
\hline 30.0 & 5.469 & 7000 & 0.236 & 0.002 & 0.148 & 0.000 & 0.000 & 0.000 & 0.000 & $\begin{array}{l}-90 \\
\end{array}$ \\
\hline 39.2 & 5.496 & 7000 & 0.270 & 0.002 & 0.149 & 0.000 & 0.000 & 0.000 & 0.000 & $\begin{array}{l}-90 \\
\end{array}$ \\
\hline 49.6 & 5.517 & 7000 & 0.304 & 0.002 & 0.149 & 0.000 & 0.000 & 0.000 & 0.000 & -90 \\
\hline 61.3 & 5.533 & 7000 & 0.337 & 0.002 & 0.154 & 0.000 & 0.000 & 0.000 & 0.000 & -90 \\
\hline
\end{tabular}


APPENDIX C. ROTOR PERFORMANCE PREDICTEIOOIIOATROTOR PERFORMANCE TABLES

C.2 T-Motor Rotor Performance Tables 
T-Motor 18x6.1 performance data table at $\theta=0 \mathrm{deg}$.

\begin{tabular}{|c|c|c|c|c|c|c|c|c|c|c|}
\hline $\mathrm{q}, \mathrm{Pa}$ & T_rho, $\mathrm{N}$ & $\mathrm{RPM}$ & $\mathrm{J}_{\infty}$ & $\mathrm{CP}$ & Q_rho, Nm & Fx_rho, N & Fy_rho, N & Mx_rho, Nm & My_rho, Nm & $\theta, \operatorname{deg}$ \\
\hline 0.0 & 5.532 & 2000 & 0.000 & 0.001 & 0.126 & 0.000 & 0.000 & 0.000 & 0.000 & 0 \\
\hline 2.5 & 5.674 & 2000 & 0.131 & 0.001 & 0.117 & -0.068 & 0.018 & -0.083 & -0.130 & 0 \\
\hline 9.8 & 6.126 & 2000 & 0.262 & 0.001 & 0.112 & -0.131 & 0.023 & -0.161 & -0.192 & 0 \\
\hline 22.1 & 6.538 & 2000 & 0.394 & 0.001 & 0.118 & -0.192 & 0.003 & -0.235 & -0.184 & 0 \\
\hline 39.2 & 6.870 & 2000 & 0.525 & 0.001 & 0.118 & -0.254 & 0.016 & -0.307 & -0.145 & 0 \\
\hline 61.3 & 7.224 & 2000 & 0.656 & 0.001 & 0.121 & -0.313 & 0.003 & -0.383 & -0.120 & 0 \\
\hline 88.2 & 7.580 & 2000 & 0.787 & 0.001 & 0.126 & -0.386 & -0.005 & -0.462 & -0.094 & 0 \\
\hline 120.1 & 7.933 & 2000 & 0.919 & 0.001 & 0.128 & -0.450 & $\begin{array}{l}-0.009 \\
\end{array}$ & $\begin{array}{l}-0.539 \\
\end{array}$ & -0.078 & 0 \\
\hline 156.8 & 8.298 & 2000 & 1.050 & 0.001 & 0.130 & -0.521 & -0.003 & -0.617 & -0.069 & 0 \\
\hline 198.5 & 8.714 & 2000 & 1.181 & 0.001 & 0.134 & -0.588 & -0.009 & -0.697 & -0.074 & 0 \\
\hline 245.0 & 9.134 & 2000 & 1.312 & 0.001 & 0.135 & -0.634 & -0.003 & -0.778 & -0.058 & 0 \\
\hline 296.5 & 9.550 & 2000 & 1.444 & 0.001 & 0.134 & -0.670 & -0.010 & -0.850 & -0.067 & 0 \\
\hline 352.8 & 9.882 & 2000 & 1.575 & 0.001 & 0.128 & -0.682 & -0.006 & -0.918 & -0.050 & 0 \\
\hline 414.1 & 10.329 & 2000 & 1.706 & 0.001 & 0.130 & -0.735 & -0.003 & -0.993 & -0.045 & 0 \\
\hline 480.2 & 10.834 & 2000 & 1.837 & 0.001 & 0.134 & -0.808 & -0.006 & -1.070 & -0.060 & 0 \\
\hline 551.3 & 11.368 & 2000 & 1.969 & 0.001 & 0.138 & -0.882 & -0.009 & -1.151 & -0.060 & 0 \\
\hline 0.0 & 13.540 & 3000 & 0.000 & 0.001 & 0.280 & 0.000 & 0.000 & 0.000 & 0.000 & 0 \\
\hline 2.5 & 13.536 & 3000 & 0.087 & 0.001 & 0.274 & -0.099 & 0.067 & -0.126 & -0.119 & 0 \\
\hline 9.8 & 13.885 & 3000 & 0.175 & 0.001 & 0.263 & -0.201 & 0.108 & -0.238 & -0.245 & 0 \\
\hline 22.1 & 14.573 & 3000 & 0.262 & 0.001 & 0.255 & -0.299 & 0.117 & -0.357 & -0.339 & 0 \\
\hline 39.2 & 15.291 & 3000 & 0.350 & 0.001 & 0.261 & -0.376 & 0.071 & -0.470 & -0.380 & 0 \\
\hline 61.3 & 15.932 & 3000 & 0.437 & 0.001 & 0.256 & -0.411 & 0.102 & -0.586 & -0.368 & 0 \\
\hline 88.2 & 16.478 & 3000 & 0.525 & 0.001 & 0.250 & -0.464 & 0.122 & -0.698 & -0.338 & 0 \\
\hline 120.1 & 17.017 & 3000 & 0.612 & 0.001 & 0.245 & -0.509 & 0.121 & -0.816 & -0.309 & 0 \\
\hline 156.8 & 17.378 & 3000 & 0.700 & 0.001 & 0.239 & -0.556 & 0.117 & -0.921 & -0.280 & 0 \\
\hline 198.5 & 17.894 & 3000 & 0.787 & 0.001 & 0.238 & -0.624 & 0.117 & -1.037 & -0.262 & 0 \\
\hline 245.0 & 18.424 & 3000 & 0.875 & 0.001 & 0.238 & -0.693 & 0.110 & -1.158 & -0.221 & 0 \\
\hline 296.5 & 18.864 & 3000 & 0.962 & 0.001 & 0.237 & -0.766 & 0.100 & -1.272 & -0.207 & 0 \\
\hline 352.8 & 19.302 & 3000 & 1.050 & 0.001 & 0.236 & -0.838 & 0.084 & -1.381 & -0.193 & 0 \\
\hline 414.1 & 19.881 & 3000 & 1.137 & 0.001 & 0.234 & -0.919 & 0.093 & -1.495 & -0.207 & 0 \\
\hline 480.2 & 20.473 & 3000 & 1.225 & 0.001 & 0.236 & -0.993 & 0.099 & -1.613 & -0.201 & 0 \\
\hline 551.3 & 21.099 & 3000 & 1.312 & 0.001 & 0.238 & -1.075 & 0.090 & -1.740 & -0.164 & 0 \\
\hline 0.0 & 23.616 & 4000 & 0.000 & 0.001 & 0.492 & 0.000 & 0.000 & 0.000 & 0.000 & 0 \\
\hline 2.5 & 23.673 & 4000 & 0.066 & 0.001 & 0.484 & -0.132 & 0.072 & -0.154 & -0.200 & 0 \\
\hline 9.8 & 24.056 & 4000 & 0.131 & 0.001 & 0.468 & -0.254 & 0.144 & -0.307 & -0.374 & 0 \\
\hline 22.1 & 24.869 & 4000 & 0.197 & 0.001 & 0.453 & -0.388 & 0.190 & -0.462 & -0.525 & 0 \\
\hline 39.2 & 25.909 & 4000 & 0.262 & 0.001 & 0.436 & -0.489 & 0.266 & -0.620 & -0.630 & 0 \\
\hline 61.3 & 26.992 & 4000 & 0.328 & 0.001 & 0.420 & -0.580 & 0.348 & -0.753 & -0.702 & 0 \\
\hline 88.2 & 27.861 & 4000 & 0.394 & 0.001 & 0.404 & -0.615 & 0.426 & -0.917 & -0.680 & 0 \\
\hline 120.1 & 28.745 & 4000 & 0.459 & 0.001 & 0.393 & -0.680 & 0.459 & -1.074 & -0.652 & 0 \\
\hline 156.8 & 29.416 & 4000 & 0.525 & 0.001 & 0.383 & -0.750 & 0.484 & -1.216 & -0.607 & 0 \\
\hline 198.5 & 30.201 & 4000 & 0.591 & 0.001 & 0.377 & -0.826 & 0.487 & -1.382 & -0.561 & 0 \\
\hline 245.0 & 30.978 & 4000 & 0.656 & 0.001 & 0.374 & -0.900 & 0.475 & -1.528 & -0.525 & 0 \\
\hline 296.5 & 31.541 & 4000 & 0.722 & 0.001 & 0.370 & -0.972 & 0.448 & -1.671 & -0.500 & 0 \\
\hline 352.8 & 32.241 & 4000 & 0.787 & 0.001 & 0.370 & $\begin{array}{l}-1.042 \\
\end{array}$ & 0.418 & $\begin{array}{l}-1.829 \\
\end{array}$ & -0.476 & 0 \\
\hline 414.1 & 32.985 & 4000 & 0.853 & 0.001 & 0.369 & -1.133 & 0.401 & -1.995 & -0.451 & 0 \\
\hline 480.2 & 33.676 & 4000 & 0.919 & 0.001 & 0.369 & -1.217 & 0.371 & -2.157 & -0.399 & 0 \\
\hline 551.3 & 34.339 & 4000 & 0.984 & 0.001 & 0.371 & -1.302 & 0.353 & -2.308 & -0.378 & 0 \\
\hline
\end{tabular}


T-Motor 18x6.1 performance data table at $\theta=5 \mathrm{deg}$.

\begin{tabular}{|c|c|c|c|c|c|c|c|c|c|c|}
\hline $\mathrm{q}, \mathrm{Pa}$ & T_rho, $\mathrm{N}$ & $\mathrm{RPM}$ & $\mathrm{J}_{\infty}$ & $\mathrm{CP}$ & Q_rho, Nm & Fx_rho, N & Fy_rho, N & Mx_rho, Nm & My_rho, Nm & $\theta, \operatorname{deg}$ \\
\hline 0.0 & 5.532 & 2000 & 0.000 & 0.001 & 0.126 & 0.000 & 0.000 & 0.000 & 0.000 & 5 \\
\hline 2.5 & 5.591 & 2000 & 0.131 & 0.001 & 0.118 & -0.069 & 0.014 & -0.083 & -0.126 & 5 \\
\hline 9.8 & 5.958 & 2000 & 0.262 & 0.001 & 0.112 & -0.135 & 0.027 & -0.162 & -0.182 & 5 \\
\hline 22.1 & 6.338 & 2000 & 0.394 & 0.001 & 0.113 & -0.193 & 0.024 & -0.238 & -0.184 & 5 \\
\hline 39.2 & 6.655 & 2000 & 0.525 & 0.001 & 0.115 & -0.251 & 0.020 & -0.311 & -0.169 & 5 \\
\hline 61.3 & 6.921 & 2000 & 0.656 & 0.001 & 0.117 & -0.315 & 0.026 & -0.386 & -0.152 & 5 \\
\hline 88.2 & 7.181 & 2000 & 0.787 & 0.001 & 0.118 & -0.375 & 0.024 & -0.466 & -0.139 & 5 \\
\hline 120.1 & 7.460 & 2000 & 0.919 & 0.001 & 0.121 & -0.440 & 0.018 & $\begin{array}{l}-0.543 \\
\end{array}$ & -0.128 & 5 \\
\hline 156.8 & 7.732 & 2000 & 1.050 & 0.001 & 0.125 & -0.519 & 0.012 & -0.616 & -0.121 & 5 \\
\hline 198.5 & 8.038 & 2000 & 1.181 & 0.001 & 0.128 & -0.593 & 0.017 & -0.689 & -0.118 & 5 \\
\hline 245.0 & 8.350 & 2000 & 1.312 & 0.001 & 0.131 & -0.660 & 0.010 & -0.764 & -0.106 & 5 \\
\hline 296.5 & 8.679 & 2000 & 1.444 & 0.001 & 0.133 & -0.717 & 0.009 & -0.836 & -0.101 & 5 \\
\hline 352.8 & 9.031 & 2000 & 1.575 & 0.001 & 0.135 & -0.776 & 0.010 & -0.907 & -0.096 & 5 \\
\hline 414.1 & 9.419 & 2000 & 1.706 & 0.001 & 0.138 & -0.841 & 0.007 & -0.967 & -0.105 & 5 \\
\hline 480.2 & 9.826 & 2000 & 1.837 & 0.001 & 0.142 & -0.908 & 0.013 & -1.037 & -0.087 & 5 \\
\hline 551.3 & 10.258 & 2000 & 1.969 & 0.001 & 0.147 & -0.996 & 0.011 & -1.099 & -0.099 & 5 \\
\hline 0.0 & 13.540 & 3000 & 0.000 & 0.001 & 0.280 & 0.000 & 0.000 & 0.000 & 0.000 & 5 \\
\hline 2.5 & 13.444 & 3000 & 0.087 & 0.001 & 0.275 & -0.101 & 0.063 & -0.126 & -0.117 & 5 \\
\hline 9.8 & 13.642 & 3000 & 0.175 & 0.001 & 0.265 & -0.198 & 0.104 & -0.237 & -0.228 & 5 \\
\hline 22.1 & 14.167 & 3000 & 0.262 & 0.001 & 0.257 & -0.299 & 0.117 & -0.355 & -0.308 & 5 \\
\hline 39.2 & 14.745 & 3000 & 0.350 & 0.001 & 0.256 & -0.387 & 0.104 & -0.470 & -0.338 & 5 \\
\hline 61.3 & 15.298 & 3000 & 0.437 & 0.001 & 0.257 & -0.482 & 0.110 & -0.585 & -0.348 & 5 \\
\hline 88.2 & 15.736 & 3000 & 0.525 & 0.001 & 0.254 & -0.527 & 0.114 & -0.704 & -0.324 & 5 \\
\hline 120.1 & 16.172 & 3000 & 0.612 & 0.001 & 0.251 & -0.599 & 0.134 & -0.820 & -0.306 & 5 \\
\hline 156.8 & 16.498 & 3000 & 0.700 & 0.001 & 0.250 & -0.659 & 0.138 & -0.924 & -0.290 & 5 \\
\hline 198.5 & 16.867 & 3000 & 0.787 & 0.001 & 0.251 & -0.738 & 0.125 & -1.046 & -0.272 & 5 \\
\hline 245.0 & 17.266 & 3000 & 0.875 & 0.001 & 0.253 & -0.817 & 0.128 & -1.161 & -0.255 & 5 \\
\hline 296.5 & 17.678 & 3000 & 0.962 & 0.001 & 0.255 & -0.908 & 0.125 & -1.276 & -0.240 & 5 \\
\hline 352.8 & 18.005 & 3000 & 1.050 & 0.001 & 0.257 & -0.976 & 0.115 & -1.381 & -0.229 & 5 \\
\hline 414.1 & 18.438 & 3000 & 1.137 & 0.001 & 0.261 & -1.070 & 0.110 & -1.491 & -0.223 & 5 \\
\hline 480.2 & 18.868 & 3000 & 1.225 & 0.001 & 0.265 & -1.170 & 0.108 & -1.598 & -0.220 & 5 \\
\hline 551.3 & 19.332 & 3000 & 1.312 & 0.001 & 0.269 & -1.269 & 0.103 & -1.709 & -0.209 & 5 \\
\hline 0.0 & 23.616 & 4000 & 0.000 & 0.001 & 0.492 & 0.000 & 0.000 & 0.000 & 0.000 & 5 \\
\hline 2.5 & 23.543 & 4000 & 0.066 & 0.001 & 0.485 & -0.133 & 0.067 & -0.153 & -0.198 & 5 \\
\hline 9.8 & 23.768 & 4000 & 0.131 & 0.001 & 0.472 & -0.259 & 0.129 & -0.305 & -0.360 & 5 \\
\hline 22.1 & 24.393 & 4000 & 0.197 & 0.001 & 0.459 & -0.396 & 0.168 & -0.460 & -0.491 & 5 \\
\hline 39.2 & 25.179 & 4000 & 0.262 & 0.001 & 0.449 & -0.513 & 0.205 & -0.614 & -0.576 & 5 \\
\hline 61.3 & 26.056 & 4000 & 0.328 & 0.001 & 0.438 & -0.611 & 0.262 & -0.767 & -0.614 & 5 \\
\hline 88.2 & 26.890 & 4000 & 0.394 & 0.001 & 0.431 & -0.693 & 0.305 & -0.919 & -0.626 & 5 \\
\hline 120.1 & 27.631 & 4000 & 0.459 & 0.001 & 0.425 & -0.756 & 0.331 & -1.080 & -0.618 & 5 \\
\hline 156.8 & 28.160 & 4000 & 0.525 & 0.001 & 0.421 & -0.830 & 0.347 & -1.217 & -0.585 & 5 \\
\hline 198.5 & 28.796 & 4000 & 0.591 & 0.001 & 0.421 & -0.927 & 0.349 & -1.368 & -0.556 & 5 \\
\hline 245.0 & 29.398 & 4000 & 0.656 & 0.001 & 0.422 & -1.022 & 0.359 & -1.517 & -0.522 & 5 \\
\hline 296.5 & 29.825 & 4000 & 0.722 & 0.001 & 0.421 & -1.102 & 0.340 & -1.657 & -0.501 & 5 \\
\hline 352.8 & 30.351 & 4000 & 0.787 & 0.001 & 0.423 & -1.206 & 0.331 & $\begin{array}{l}-1.824 \\
\end{array}$ & -0.477 & 5 \\
\hline 414.1 & 30.881 & 4000 & 0.853 & 0.001 & 0.427 & -1.310 & 0.316 & $\begin{array}{l}-1.979 \\
\end{array}$ & -0.455 & 5 \\
\hline 480.2 & 31.421 & 4000 & 0.919 & 0.001 & 0.431 & -1.419 & 0.299 & -2.121 & -0.433 & 5 \\
\hline 551.3 & 32.077 & 4000 & 0.984 & 0.001 & 0.436 & -1.525 & 0.285 & -2.281 & -0.413 & 5 \\
\hline
\end{tabular}


T-Motor 18x6.1 performance data table at $\theta=10 \mathrm{deg}$.

\begin{tabular}{|c|c|c|c|c|c|c|c|c|c|c|}
\hline $\mathrm{q}, \mathrm{Pa}$ & T_rho, $\mathrm{N}$ & $\mathrm{RPM}$ & $\mathrm{J}_{\infty}$ & $\mathrm{CP}$ & Q_rho, Nm & Fx_rho, N & Fy_rho, N & Mx_rho, Nm & My_rho, Nm & $\theta, \operatorname{deg}$ \\
\hline 0.0 & 5.532 & 2000 & 0.000 & 0.001 & 0.126 & 0.000 & 0.000 & 0.000 & 0.000 & 10 \\
\hline 2.5 & 5.501 & 2000 & 0.131 & 0.001 & 0.118 & -0.071 & 0.010 & -0.083 & -0.122 & 10 \\
\hline 9.8 & 5.751 & 2000 & 0.262 & 0.001 & 0.113 & -0.137 & 0.017 & -0.162 & -0.170 & 10 \\
\hline 22.1 & 6.020 & 2000 & 0.394 & 0.001 & 0.113 & -0.200 & 0.021 & -0.238 & -0.179 & 10 \\
\hline 39.2 & 6.214 & 2000 & 0.525 & 0.001 & 0.114 & -0.262 & 0.023 & -0.317 & -0.164 & 10 \\
\hline 61.3 & 6.346 & 2000 & 0.656 & 0.001 & 0.116 & $\begin{array}{c}-0.331 \\
\end{array}$ & 0.018 & -0.385 & -0.146 & 10 \\
\hline 88.2 & 6.445 & 2000 & 0.787 & 0.001 & 0.119 & -0.404 & 0.011 & -0.459 & -0.131 & 10 \\
\hline 120.1 & 6.531 & 2000 & 0.919 & 0.001 & 0.122 & -0.482 & 0.001 & -0.537 & -0.120 & 10 \\
\hline 156.8 & 6.644 & 2000 & 1.050 & 0.001 & 0.124 & -0.563 & -0.005 & -0.607 & -0.112 & 10 \\
\hline 198.5 & 6.770 & 2000 & 1.181 & 0.001 & 0.127 & -0.651 & -0.013 & -0.679 & -0.105 & 10 \\
\hline 245.0 & 6.979 & 2000 & 1.312 & 0.001 & 0.132 & -0.740 & -0.022 & -0.736 & -0.099 & 10 \\
\hline 296.5 & 7.224 & 2000 & 1.444 & 0.001 & 0.136 & -0.807 & -0.016 & -0.791 & -0.087 & 10 \\
\hline 352.8 & 7.528 & 2000 & 1.575 & 0.001 & 0.144 & -0.896 & -0.015 & -0.839 & -0.077 & 10 \\
\hline 414.1 & 7.844 & 2000 & 1.706 & 0.001 & 0.150 & -0.988 & -0.021 & -0.889 & -0.071 & 10 \\
\hline 480.2 & 8.108 & 2000 & 1.837 & 0.001 & 0.155 & -1.075 & -0.017 & -0.938 & -0.067 & 10 \\
\hline 551.3 & 8.407 & 2000 & 1.969 & 0.002 & 0.161 & -1.187 & -0.020 & -0.998 & -0.072 & 10 \\
\hline 0.0 & 13.540 & 3000 & 0.000 & 0.001 & 0.280 & 0.000 & 0.000 & 0.000 & 0.000 & 10 \\
\hline 2.5 & 13.345 & 3000 & 0.087 & 0.001 & 0.276 & -0.103 & 0.057 & -0.126 & -0.115 & 10 \\
\hline 9.8 & 13.407 & 3000 & 0.175 & 0.001 & 0.268 & -0.198 & 0.091 & -0.231 & -0.229 & 10 \\
\hline 22.1 & 13.731 & 3000 & 0.262 & 0.001 & 0.261 & -0.299 & 0.100 & -0.348 & -0.295 & 10 \\
\hline 39.2 & 14.107 & 3000 & 0.350 & 0.001 & 0.259 & -0.394 & 0.103 & -0.461 & -0.319 & 10 \\
\hline 61.3 & 14.448 & 3000 & 0.437 & 0.001 & 0.260 & -0.496 & 0.096 & -0.583 & -0.295 & 10 \\
\hline 88.2 & 14.740 & 3000 & 0.525 & 0.001 & 0.261 & -0.590 & 0.097 & -0.695 & -0.297 & 10 \\
\hline 120.1 & 14.965 & 3000 & 0.612 & 0.001 & 0.263 & -0.675 & 0.096 & -0.803 & -0.274 & 10 \\
\hline 156.8 & 15.109 & 3000 & 0.700 & 0.001 & 0.265 & -0.767 & 0.081 & -0.925 & -0.259 & 10 \\
\hline 198.5 & 15.276 & 3000 & 0.787 & 0.001 & 0.269 & -0.865 & 0.071 & -1.040 & -0.237 & 10 \\
\hline 245.0 & 15.416 & 3000 & 0.875 & 0.001 & 0.272 & -0.968 & 0.055 & $\begin{array}{l}-1.132 \\
\end{array}$ & -0.221 & 10 \\
\hline 296.5 & 15.587 & 3000 & 0.962 & 0.001 & 0.275 & -1.080 & 0.049 & -1.243 & -0.210 & 10 \\
\hline 352.8 & 15.755 & 3000 & 1.050 & 0.001 & 0.278 & -1.178 & 0.034 & -1.347 & -0.201 & 10 \\
\hline 414.1 & 15.977 & 3000 & 1.137 & 0.001 & 0.283 & -1.297 & 0.022 & -1.471 & -0.198 & 10 \\
\hline 480.2 & 16.208 & 3000 & 1.225 & 0.001 & 0.289 & -1.422 & 0.013 & -1.548 & -0.192 & 10 \\
\hline 551.3 & 16.507 & 3000 & 1.312 & 0.001 & 0.295 & -1.537 & 0.006 & -1.653 & -0.194 & 10 \\
\hline 0.0 & 23.616 & 4000 & 0.000 & 0.001 & 0.492 & 0.000 & 0.000 & 0.000 & 0.000 & 10 \\
\hline 2.5 & 23.411 & 4000 & 0.066 & 0.001 & 0.487 & -0.133 & 0.062 & -0.152 & -0.193 & 10 \\
\hline 9.8 & 23.471 & 4000 & 0.131 & 0.001 & 0.475 & -0.260 & 0.111 & -0.301 & -0.345 & 10 \\
\hline 22.1 & 23.869 & 4000 & 0.197 & 0.001 & 0.464 & -0.386 & 0.148 & -0.448 & -0.487 & 10 \\
\hline 39.2 & 24.413 & 4000 & 0.262 & 0.001 & 0.458 & -0.522 & 0.162 & -0.599 & -0.556 & 10 \\
\hline 61.3 & 25.011 & 4000 & 0.328 & 0.001 & 0.454 & -0.644 & 0.183 & -0.746 & -0.582 & 10 \\
\hline 88.2 & 25.585 & 4000 & 0.394 & 0.001 & 0.452 & -0.738 & 0.192 & -0.902 & -0.548 & 10 \\
\hline 120.1 & 26.068 & 4000 & 0.459 & 0.001 & 0.451 & -0.842 & 0.207 & -1.032 & -0.548 & 10 \\
\hline 156.8 & 26.499 & 4000 & 0.525 & 0.001 & 0.453 & -0.954 & 0.204 & -1.192 & -0.541 & 10 \\
\hline 198.5 & 26.898 & 4000 & 0.591 & 0.001 & 0.456 & -1.069 & 0.203 & -1.327 & -0.506 & 10 \\
\hline 245.0 & 27.184 & 4000 & 0.656 & 0.001 & 0.460 & -1.178 & 0.196 & -1.493 & -0.476 & 10 \\
\hline 296.5 & 27.414 & 4000 & 0.722 & 0.001 & 0.465 & -1.302 & 0.172 & -1.647 & -0.449 & 10 \\
\hline 352.8 & 27.625 & 4000 & 0.787 & 0.001 & 0.470 & -1.415 & 0.176 & $\begin{array}{l}-1.793 \\
\end{array}$ & -0.423 & 10 \\
\hline 414.1 & 27.852 & 4000 & 0.853 & 0.001 & 0.475 & -1.556 & 0.155 & -1.941 & -0.409 & 10 \\
\hline 480.2 & 28.089 & 4000 & 0.919 & 0.001 & 0.481 & -1.698 & 0.137 & -2.085 & -0.398 & 10 \\
\hline 551.3 & 28.455 & 4000 & 0.984 & 0.001 & 0.488 & -1.847 & 0.119 & -2.233 & -0.387 & 10 \\
\hline
\end{tabular}


T-Motor 18x6.1 performance data table at $\theta=20 \mathrm{deg}$.

\begin{tabular}{|c|c|c|c|c|c|c|c|c|c|c|}
\hline $\mathrm{q}, \mathrm{Pa}$ & T_rho, $\mathrm{N}$ & $\mathrm{RPM}$ & $\mathrm{J}_{\infty}$ & $\mathrm{CP}$ & Q_rho, Nm & Fx_rho, N & Fy_rho, N & Mx_rho, Nm & My_rho, Nm & $\theta, \operatorname{deg}$ \\
\hline 0.0 & 5.532 & 2000 & 0.000 & 0.001 & 0.126 & 0.000 & 0.000 & 0.000 & 0.000 & 20 \\
\hline 2.5 & 5.318 & 2000 & 0.131 & 0.001 & 0.120 & -0.071 & 0.000 & -0.080 & -0.120 & 20 \\
\hline 9.8 & 5.299 & 2000 & 0.262 & 0.001 & 0.116 & -0.140 & -0.003 & -0.155 & -0.153 & 20 \\
\hline 22.1 & 5.248 & 2000 & 0.394 & 0.001 & 0.115 & -0.218 & -0.011 & -0.231 & -0.154 & 20 \\
\hline 39.2 & 5.091 & 2000 & 0.525 & 0.001 & 0.116 & -0.300 & -0.025 & -0.308 & -0.140 & 20 \\
\hline 61.3 & 4.822 & 2000 & 0.656 & 0.001 & 0.115 & -0.393 & -0.035 & -0.386 & -0.111 & 20 \\
\hline 88.2 & 4.490 & 2000 & 0.787 & 0.001 & 0.112 & -0.491 & -0.044 & -0.453 & -0.105 & 20 \\
\hline 120.1 & 4.249 & 2000 & 0.919 & 0.001 & 0.111 & $\begin{array}{l}-0.579 \\
\end{array}$ & -0.044 & $\begin{array}{l}-0.509 \\
\end{array}$ & -0.075 & 20 \\
\hline 156.8 & 4.080 & 2000 & 1.050 & 0.001 & 0.112 & -0.649 & -0.042 & -0.536 & -0.077 & 20 \\
\hline 198.5 & 3.984 & 2000 & 1.181 & 0.001 & 0.115 & -0.722 & -0.033 & -0.555 & -0.065 & 20 \\
\hline 245.0 & 3.892 & 2000 & 1.312 & 0.001 & 0.118 & -0.798 & -0.024 & -0.567 & -0.035 & 20 \\
\hline 296.5 & 3.746 & 2000 & 1.444 & 0.001 & 0.120 & -0.876 & -0.016 & -0.571 & -0.041 & 20 \\
\hline 352.8 & 3.517 & 2000 & 1.575 & 0.001 & 0.121 & -0.944 & -0.019 & -0.565 & -0.020 & 20 \\
\hline 414.1 & 3.311 & 2000 & 1.706 & 0.001 & 0.121 & -1.020 & -0.012 & -0.563 & -0.012 & 20 \\
\hline 480.2 & 3.128 & 2000 & 1.837 & 0.001 & 0.122 & -1.105 & -0.013 & -0.564 & -0.007 & 20 \\
\hline 551.3 & 2.985 & 2000 & 1.969 & 0.001 & 0.126 & -1.194 & -0.003 & -0.567 & -0.001 & 20 \\
\hline 0.0 & 13.540 & 3000 & 0.000 & 0.001 & 0.280 & 0.000 & 0.000 & 0.000 & 0.000 & 20 \\
\hline 2.5 & 13.164 & 3000 & 0.087 & 0.001 & 0.278 & -0.102 & 0.046 & -0.122 & -0.126 & 20 \\
\hline 9.8 & 12.930 & 3000 & 0.175 & 0.001 & 0.274 & -0.199 & 0.064 & -0.226 & -0.203 & 20 \\
\hline 22.1 & 12.838 & 3000 & 0.262 & 0.001 & 0.269 & -0.301 & 0.062 & -0.333 & -0.245 & 20 \\
\hline 39.2 & 12.755 & 3000 & 0.350 & 0.001 & 0.268 & -0.411 & 0.048 & -0.443 & -0.268 & 20 \\
\hline 61.3 & 12.627 & 3000 & 0.437 & 0.001 & 0.269 & -0.531 & 0.031 & -0.555 & -0.262 & 20 \\
\hline 88.2 & 12.400 & 3000 & 0.525 & 0.001 & 0.271 & -0.645 & 0.008 & -0.664 & -0.249 & 20 \\
\hline 120.1 & 12.131 & 3000 & 0.612 & 0.001 & 0.272 & -0.776 & -0.009 & -0.789 & -0.216 & 20 \\
\hline 156.8 & 11.761 & 3000 & 0.700 & 0.001 & 0.272 & -0.916 & -0.030 & -0.889 & -0.209 & 20 \\
\hline 198.5 & 11.354 & 3000 & 0.787 & 0.001 & 0.271 & -1.060 & -0.051 & -0.973 & -0.220 & 20 \\
\hline 245.0 & 10.954 & 3000 & 0.875 & 0.001 & 0.271 & $\begin{array}{l}-1.198 \\
\end{array}$ & -0.066 & -1.067 & -0.178 & 20 \\
\hline 296.5 & 10.604 & 3000 & 0.962 & 0.001 & 0.271 & -1.325 & -0.060 & -1.137 & -0.151 & 20 \\
\hline 352.8 & 10.292 & 3000 & 1.050 & 0.001 & 0.274 & -1.443 & -0.060 & -1.181 & -0.128 & 20 \\
\hline 414.1 & 10.066 & 3000 & 1.137 & 0.001 & 0.277 & -1.557 & -0.057 & -1.217 & -0.144 & 20 \\
\hline 480.2 & 9.761 & 3000 & 1.225 & 0.001 & 0.281 & -1.658 & -0.062 & -1.227 & -0.136 & 20 \\
\hline 551.3 & 9.478 & 3000 & 1.312 & 0.001 & 0.281 & -1.776 & -0.072 & -1.247 & -0.137 & 20 \\
\hline 0.0 & 23.616 & 4000 & 0.000 & 0.001 & 0.492 & 0.000 & 0.000 & 0.000 & 0.000 & 20 \\
\hline 2.5 & 23.131 & 4000 & 0.066 & 0.001 & 0.489 & -0.131 & 0.048 & -0.144 & -0.210 & 20 \\
\hline 9.8 & 22.873 & 4000 & 0.131 & 0.001 & 0.483 & -0.256 & 0.078 & -0.284 & -0.345 & 20 \\
\hline 22.1 & 22.782 & 4000 & 0.197 & 0.001 & 0.476 & -0.385 & 0.092 & -0.426 & -0.430 & 20 \\
\hline 39.2 & 22.819 & 4000 & 0.262 & 0.001 & 0.473 & -0.514 & 0.093 & -0.566 & -0.470 & 20 \\
\hline 61.3 & 22.830 & 4000 & 0.328 & 0.001 & 0.473 & -0.652 & 0.077 & -0.714 & -0.451 & 20 \\
\hline 88.2 & 22.810 & 4000 & 0.394 & 0.001 & 0.475 & -0.798 & 0.064 & -0.840 & -0.493 & 20 \\
\hline 120.1 & 22.657 & 4000 & 0.459 & 0.001 & 0.479 & -0.952 & 0.040 & -0.991 & -0.448 & 20 \\
\hline 156.8 & 22.395 & 4000 & 0.525 & 0.001 & 0.483 & -1.099 & 0.011 & -1.128 & -0.421 & 20 \\
\hline 198.5 & 22.141 & 4000 & 0.591 & 0.001 & 0.488 & -1.259 & -0.012 & -1.257 & -0.400 & 20 \\
\hline 245.0 & 21.797 & 4000 & 0.656 & 0.001 & 0.491 & -1.418 & -0.037 & -1.369 & -0.432 & 20 \\
\hline 296.5 & 21.489 & 4000 & 0.722 & 0.001 & 0.496 & -1.594 & -0.063 & -1.491 & -0.400 & 20 \\
\hline 352.8 & 20.944 & 4000 & 0.787 & 0.001 & 0.495 & -1.775 & -0.088 & -1.645 & $\begin{array}{c}-0.339 \\
\end{array}$ & 20 \\
\hline 414.1 & 20.365 & 4000 & 0.853 & 0.001 & 0.491 & -1.971 & -0.123 & -1.761 & -0.371 & 20 \\
\hline 480.2 & 19.801 & 4000 & 0.919 & 0.001 & 0.489 & -2.157 & -0.132 & -1.879 & -0.353 & 20 \\
\hline 551.3 & 19.335 & 4000 & 0.984 & 0.001 & 0.489 & -2.352 & -0.144 & -2.001 & -0.278 & 20 \\
\hline
\end{tabular}


T-Motor 18x6.1 performance data table at $\theta=40 \mathrm{deg}$.

\begin{tabular}{|c|c|c|c|c|c|c|c|c|c|c|}
\hline $\mathrm{q}, \mathrm{Pa}$ & T_rho, $\mathrm{N}$ & $\mathrm{RPM}$ & $\mathrm{J}_{\infty}$ & $\mathrm{CP}$ & Q_rho, Nm & Fx_rho, N & Fy_rho, N & Mx_rho, Nm & My_rho, Nm & $\theta, \operatorname{deg}$ \\
\hline 0.0 & 5.532 & 2000 & 0.000 & 0.001 & 0.126 & 0.000 & 0.000 & 0.000 & 0.000 & 40 \\
\hline 2.5 & 4.917 & 2000 & 0.131 & 0.001 & 0.122 & -0.066 & -0.015 & -0.067 & -0.090 & 40 \\
\hline 9.8 & 4.297 & 2000 & 0.262 & 0.001 & 0.117 & -0.140 & -0.037 & -0.130 & -0.098 & 40 \\
\hline 22.1 & 3.466 & 2000 & 0.394 & 0.001 & 0.108 & -0.232 & -0.050 & -0.188 & -0.083 & 40 \\
\hline 39.2 & 2.411 & 2000 & 0.525 & 0.001 & 0.090 & -0.325 & -0.045 & -0.256 & -0.056 & 40 \\
\hline 61.3 & 1.291 & 2000 & 0.656 & 0.001 & 0.069 & -0.365 & -0.021 & -0.283 & -0.024 & 40 \\
\hline 88.2 & 0.296 & 2000 & 0.787 & 0.000 & 0.052 & -0.326 & -0.004 & -0.232 & -0.002 & 40 \\
\hline 120.1 & -0.665 & 2000 & 0.919 & 0.000 & 0.037 & -0.259 & 0.000 & -0.148 & -0.001 & 40 \\
\hline 156.8 & -1.728 & 2000 & 1.050 & 0.000 & 0.021 & -0.203 & 0.004 & -0.067 & -0.002 & 40 \\
\hline 198.5 & -2.824 & 2000 & 1.181 & 0.000 & 0.004 & -0.132 & 0.005 & 0.015 & -0.001 & 40 \\
\hline 245.0 & -3.667 & 2000 & 1.312 & 0.000 & 0.001 & -0.152 & -0.002 & 0.065 & -0.002 & 40 \\
\hline 296.5 & -4.212 & 2000 & 1.444 & 0.000 & 0.016 & -0.302 & -0.003 & 0.063 & -0.002 & 40 \\
\hline 352.8 & -4.796 & 2000 & 1.575 & 0.000 & 0.016 & -0.348 & -0.003 & 0.053 & -0.001 & 40 \\
\hline 414.1 & -5.583 & 2000 & 1.706 & 0.000 & 0.001 & -0.334 & -0.002 & 0.068 & -0.001 & 40 \\
\hline 480.2 & -6.419 & 2000 & 1.837 & 0.000 & -0.011 & -0.352 & -0.001 & 0.081 & -0.002 & 40 \\
\hline 551.3 & -7.278 & 2000 & 1.969 & 0.000 & -0.024 & -0.364 & -0.001 & 0.090 & -0.001 & 40 \\
\hline 0.0 & 13.540 & 3000 & 0.000 & 0.001 & 0.280 & 0.000 & 0.000 & 0.000 & 0.000 & 40 \\
\hline 2.5 & 12.771 & 3000 & 0.087 & 0.001 & 0.283 & -0.089 & 0.026 & -0.103 & -0.086 & 40 \\
\hline 9.8 & 11.980 & 3000 & 0.175 & 0.001 & 0.282 & -0.187 & 0.018 & -0.195 & -0.146 & 40 \\
\hline 22.1 & 11.067 & 3000 & 0.262 & 0.001 & 0.279 & -0.299 & -0.015 & -0.277 & -0.181 & 40 \\
\hline 39.2 & 10.010 & 3000 & 0.350 & 0.001 & 0.273 & -0.430 & -0.053 & -0.376 & -0.178 & 40 \\
\hline 61.3 & 8.694 & 3000 & 0.437 & 0.001 & 0.261 & -0.555 & -0.075 & -0.451 & -0.173 & 40 \\
\hline 88.2 & 7.284 & 3000 & 0.525 & 0.001 & 0.244 & -0.681 & -0.082 & -0.539 & -0.124 & 40 \\
\hline 120.1 & 5.722 & 3000 & 0.612 & 0.001 & 0.221 & -0.781 & -0.070 & -0.608 & -0.088 & 40 \\
\hline 156.8 & 4.049 & 3000 & 0.700 & 0.001 & 0.193 & -0.841 & -0.069 & -0.640 & -0.066 & 40 \\
\hline 198.5 & 2.414 & 3000 & 0.787 & 0.001 & 0.166 & -0.849 & -0.042 & -0.618 & -0.041 & 40 \\
\hline 245.0 & 0.769 & 3000 & 0.875 & 0.001 & 0.144 & -0.742 & -0.014 & -0.520 & -0.012 & 40 \\
\hline 296.5 & -0.704 & 3000 & 0.962 & 0.001 & 0.121 & -0.676 & 0.006 & -0.439 & 0.002 & 40 \\
\hline 352.8 & -2.579 & 3000 & 1.050 & 0.000 & 0.076 & -0.615 & -0.003 & -0.316 & -0.003 & 40 \\
\hline 414.1 & -4.288 & 3000 & 1.137 & 0.000 & 0.037 & -0.484 & -0.010 & -0.195 & -0.004 & 40 \\
\hline 480.2 & -6.048 & 3000 & 1.225 & 0.000 & -0.006 & -0.374 & -0.009 & -0.080 & -0.006 & 40 \\
\hline 551.3 & -7.628 & 3000 & 1.312 & 0.000 & -0.043 & -0.245 & -0.008 & 0.011 & -0.003 & 40 \\
\hline 0.0 & 23.616 & 4000 & 0.000 & 0.001 & 0.492 & 0.000 & 0.000 & 0.000 & 0.000 & 40 \\
\hline 2.5 & 22.575 & 4000 & 0.066 & 0.001 & 0.494 & -0.111 & 0.025 & -0.118 & -0.170 & 40 \\
\hline 9.8 & 21.612 & 4000 & 0.131 & 0.001 & 0.494 & -0.225 & 0.020 & -0.235 & -0.241 & 40 \\
\hline 22.1 & 20.621 & 4000 & 0.197 & 0.001 & 0.491 & -0.357 & -0.007 & -0.351 & -0.296 & 40 \\
\hline 39.2 & 19.508 & 4000 & 0.262 & 0.001 & 0.488 & -0.500 & -0.045 & -0.467 & -0.327 & 40 \\
\hline 61.3 & 18.238 & 4000 & 0.328 & 0.001 & 0.483 & -0.654 & -0.085 & -0.577 & -0.352 & 40 \\
\hline 88.2 & 16.734 & 4000 & 0.394 & 0.001 & 0.472 & -0.819 & -0.118 & -0.687 & -0.287 & 40 \\
\hline 120.1 & 15.061 & 4000 & 0.459 & 0.001 & 0.455 & -0.997 & -0.149 & -0.791 & -0.268 & 40 \\
\hline 156.8 & 13.197 & 4000 & 0.525 & 0.001 & 0.432 & -1.154 & -0.161 & -0.889 & -0.221 & 40 \\
\hline 198.5 & 11.181 & 4000 & 0.591 & 0.001 & 0.401 & -1.313 & -0.136 & -0.962 & -0.216 & 40 \\
\hline 245.0 & 9.084 & 4000 & 0.656 & 0.001 & 0.370 & -1.424 & -0.104 & -1.051 & -0.135 & 40 \\
\hline 296.5 & 7.012 & 4000 & 0.722 & 0.001 & 0.331 & -1.536 & -0.097 & -1.108 & -0.095 & 40 \\
\hline 352.8 & 4.902 & 4000 & 0.787 & 0.001 & 0.284 & -1.624 & -0.073 & -1.129 & -0.080 & 40 \\
\hline 414.1 & 3.040 & 4000 & 0.853 & 0.001 & 0.259 & -1.550 & -0.028 & -1.084 & $\begin{array}{l}-0.033 \\
\end{array}$ & 40 \\
\hline 480.2 & 1.209 & 4000 & 0.919 & 0.001 & 0.225 & -1.511 & -0.011 & -1.012 & -0.015 & 40 \\
\hline 551.3 & -0.119 & 4000 & 0.984 & 0.000 & 0.209 & -1.458 & 0.016 & -0.942 & 0.018 & 40 \\
\hline
\end{tabular}


T-Motor 18x6.1 performance data table at $\theta=90 \mathrm{deg}$.

\begin{tabular}{|c|c|c|c|c|c|c|c|c|c|c|}
\hline $\mathrm{q}, \mathrm{Pa}$ & T_rho, $\mathrm{N}$ & RPM & $\mathrm{J}_{\infty}$ & $\mathrm{CP}$ & Q_rho, Nm & Fx_rho, N & Fy_rho, $\mathrm{N}$ & Mx_rho, Nm & My_rho, Nm & $\theta, \mathrm{d}$ \\
\hline 0.0 & 5.532 & 2000 & 0.000 & 0.001 & 0.126 & 0.000 & 0.000 & 0.000 & 0.000 & 90 \\
\hline 2.5 & 4.298 & 2000 & 0.131 & 0.001 & 0.120 & 0.000 & 0.000 & 0.000 & 0.000 & 90 \\
\hline 9.8 & 2.933 & 2000 & 0.262 & 0.001 & 0.103 & 0.000 & 0.000 & 0.000 & 0.000 & 90 \\
\hline 22.1 & 0.974 & 2000 & 0.394 & 0.001 & 0.063 & 0.000 & 0.000 & 0.000 & 0.000 & 90 \\
\hline 39.2 & -1.699 & 2000 & 0.525 & 0.000 & 0.006 & 0.000 & 0.000 & 0.000 & 0.000 & 90 \\
\hline 61.3 & -2.646 & 2000 & 0.656 & 0.000 & 0.010 & 0.000 & 0.000 & 0.000 & 0.000 & 90 \\
\hline 88.2 & -3.123 & 2000 & 0.787 & 0.000 & 0.019 & 0.000 & 0.000 & 0.000 & 0.000 & 90 \\
\hline 120.1 & -4.133 & 2000 & 0.919 & 0.000 & -0.023 & 0.000 & 0.000 & 0.000 & 0.000 & 90 \\
\hline 156.8 & -5.218 & 2000 & 1.050 & 0.000 & -0.051 & 0.000 & 0.000 & 0.000 & 0.000 & 90 \\
\hline 198.5 & -6.417 & 2000 & 1.181 & -0.001 & -0.053 & 0.000 & 0.000 & 0.000 & 0.000 & 90 \\
\hline 245.0 & -7.702 & 2000 & 1.312 & -0.001 & -0.054 & 0.000 & 0.000 & 0.000 & 0.000 & 90 \\
\hline 296.5 & -9.079 & 2000 & 1.444 & -0.001 & -0.055 & 0.000 & 0.000 & 0.000 & 0.000 & 90 \\
\hline 352.8 & -10.553 & 2000 & 1.575 & -0.001 & -0.056 & 0.000 & 0.000 & 0.000 & 0.000 & 90 \\
\hline 414.1 & -12.126 & 2000 & 1.706 & -0.001 & -0.059 & 0.000 & 0.000 & 0.000 & 0.000 & 90 \\
\hline 480.2 & -13.804 & 2000 & 1.837 & -0.001 & -0.064 & 0.000 & 0.000 & 0.000 & 0.000 & 90 \\
\hline 551.3 & -15.588 & 2000 & 1.969 & -0.001 & -0.070 & 0.000 & 0.000 & 0.000 & 0.000 & 90 \\
\hline 0.0 & 13.540 & 3000 & 0.000 & 0.001 & 0.280 & 0.000 & 0.000 & 0.000 & 0.000 & 90 \\
\hline 2.5 & 12.194 & 3000 & 0.087 & 0.001 & 0.288 & 0.000 & 0.000 & 0.000 & 0.000 & 90 \\
\hline 9.8 & 10.529 & 3000 & 0.175 & 0.001 & 0.289 & 0.000 & 0.000 & 0.000 & 0.000 & 90 \\
\hline 22.1 & 8.475 & 3000 & 0.262 & 0.001 & 0.269 & 0.000 & 0.000 & 0.000 & 0.000 & 90 \\
\hline 39.2 & 6.054 & 3000 & 0.350 & 0.001 & 0.237 & 0.000 & 0.000 & 0.000 & 0.000 & 90 \\
\hline 61.3 & 3.604 & 3000 & 0.437 & 0.001 & 0.191 & 0.000 & 0.000 & 0.000 & 0.000 & 90 \\
\hline 88.2 & 0.042 & 3000 & 0.525 & 0.000 & 0.106 & 0.000 & 0.000 & 0.000 & 0.000 & 90 \\
\hline 120.1 & -5.448 & 3000 & 0.612 & 0.000 & -0.005 & 0.000 & 0.000 & 0.000 & 0.000 & 90 \\
\hline 156.8 & -5.979 & 3000 & 0.700 & 0.000 & 0.094 & 0.000 & 0.000 & 0.000 & 0.000 & 90 \\
\hline 198.5 & -7.387 & 3000 & 0.787 & 0.000 & 0.032 & 0.000 & 0.000 & 0.000 & 0.000 & 90 \\
\hline 245.0 & -8.955 & 3000 & 0.875 & 0.000 & $\begin{array}{l}-0.039 \\
\end{array}$ & 0.000 & 0.000 & 0.000 & 0.000 & 90 \\
\hline 296.5 & -10.589 & 3000 & 0.962 & 0.000 & -0.097 & 0.000 & 0.000 & 0.000 & 0.000 & 90 \\
\hline 352.8 & -12.299 & 3000 & 1.050 & -0.001 & -0.144 & 0.000 & 0.000 & 0.000 & 0.000 & 90 \\
\hline 414.1 & -14.121 & 3000 & 1.137 & -0.001 & -0.165 & 0.000 & 0.000 & 0.000 & 0.000 & 90 \\
\hline 480.2 & -16.038 & 3000 & 1.225 & -0.001 & -0.175 & 0.000 & 0.000 & 0.000 & 0.000 & 90 \\
\hline 551.3 & -18.039 & 3000 & 1.312 & -0.001 & -0.185 & 0.000 & 0.000 & 0.000 & 0.000 & 90 \\
\hline 0.0 & 23.616 & 4000 & 0.000 & 0.001 & 0.492 & 0.000 & 0.000 & 0.000 & 0.000 & 90 \\
\hline 2.5 & 21.820 & 4000 & 0.066 & 0.001 & 0.499 & 0.000 & 0.000 & 0.000 & 0.000 & 90 \\
\hline 9.8 & 19.795 & 4000 & 0.131 & 0.001 & 0.501 & 0.000 & 0.000 & 0.000 & 0.000 & 90 \\
\hline 22.1 & 17.427 & 4000 & 0.197 & 0.001 & 0.491 & 0.000 & 0.000 & 0.000 & 0.000 & 90 \\
\hline 39.2 & 14.678 & 4000 & 0.262 & 0.001 & 0.463 & 0.000 & 0.000 & 0.000 & 0.000 & 90 \\
\hline 61.3 & 11.472 & 4000 & 0.328 & 0.001 & 0.418 & 0.000 & 0.000 & 0.000 & 0.000 & 90 \\
\hline 88.2 & 7.915 & 4000 & 0.394 & 0.001 & 0.349 & 0.000 & 0.000 & 0.000 & 0.000 & 90 \\
\hline 120.1 & 4.086 & 4000 & 0.459 & 0.001 & 0.265 & 0.000 & 0.000 & 0.000 & 0.000 & 90 \\
\hline 156.8 & 0.330 & 4000 & 0.525 & 0.000 & 0.167 & 0.000 & 0.000 & 0.000 & 0.000 & 90 \\
\hline 198.5 & -5.955 & 4000 & 0.591 & 0.000 & -0.010 & 0.000 & 0.000 & 0.000 & 0.000 & 90 \\
\hline 245.0 & -10.563 & 4000 & 0.656 & 0.000 & -0.094 & 0.000 & 0.000 & 0.000 & 0.000 & 90 \\
\hline 296.5 & -12.624 & 4000 & 0.722 & 0.000 & -0.087 & 0.000 & 0.000 & 0.000 & 0.000 & 90 \\
\hline 352.8 & -13.841 & 4000 & 0.787 & 0.000 & -0.023 & 0.000 & 0.000 & 0.000 & 0.000 & 90 \\
\hline 414.1 & -15.768 & 4000 & 0.853 & 0.000 & -0.106 & 0.000 & 0.000 & 0.000 & 0.000 & 90 \\
\hline 480.2 & -17.980 & 4000 & 0.919 & 0.000 & -0.193 & 0.000 & 0.000 & 0.000 & 0.000 & 90 \\
\hline 551.3 & -20.265 & 4000 & 0.984 & -0.001 & -0.263 & 0.000 & 0.000 & 0.000 & 0.000 & 90 \\
\hline
\end{tabular}


T-Motor $18 \times 6.1$ performance data table at $\theta=-5 \mathrm{deg}$.

\begin{tabular}{|c|c|c|c|c|c|c|c|c|c|c|}
\hline $\mathrm{q}, \mathrm{Pa}$ & T_rho, $\mathrm{N}$ & $\mathrm{RPM}$ & $\mathrm{J}_{\infty}$ & $\mathrm{CP}$ & Q_rho, Nm & Fx_rho, N & Fy_rho, N & Mx_rho, Nm & My_rho, Nm & $\theta, \operatorname{deg}$ \\
\hline 0.0 & 5.532 & 2000 & 0.000 & 0.001 & 0.126 & 0.000 & 0.000 & 0.000 & 0.000 & -5 \\
\hline 2.5 & 5.754 & 2000 & 0.131 & 0.001 & 0.117 & -0.069 & 0.020 & -0.083 & -0.134 & -5 \\
\hline 9.8 & 6.254 & 2000 & 0.262 & 0.001 & 0.114 & -0.128 & 0.011 & -0.159 & -0.199 & -5 \\
\hline 22.1 & 6.582 & 2000 & 0.394 & 0.001 & 0.117 & -0.180 & 0.013 & -0.231 & -0.154 & -5 \\
\hline 39.2 & 6.888 & 2000 & 0.525 & 0.001 & 0.122 & -0.260 & 0.000 & -0.306 & -0.091 & -5 \\
\hline 61.3 & 7.191 & 2000 & 0.656 & 0.001 & 0.121 & -0.318 & -0.011 & -0.379 & -0.037 & -5 \\
\hline 88.2 & 7.467 & 2000 & 0.787 & 0.001 & 0.126 & -0.397 & -0.025 & -0.453 & -0.011 & -5 \\
\hline 120.1 & 7.784 & 2000 & 0.919 & 0.001 & 0.129 & -0.467 & -0.038 & $\begin{array}{l}-0.534 \\
\end{array}$ & 0.017 & -5 \\
\hline 156.8 & 8.089 & 2000 & 1.050 & 0.001 & 0.140 & -0.569 & 0.046 & -0.610 & -0.047 & -5 \\
\hline 198.5 & 8.366 & 2000 & 1.181 & 0.001 & 0.145 & -0.644 & 0.052 & -0.683 & -0.047 & -5 \\
\hline 245.0 & 8.670 & 2000 & 1.312 & 0.001 & 0.147 & -0.689 & 0.055 & -0.760 & -0.058 & -5 \\
\hline 296.5 & 8.931 & 2000 & 1.444 & 0.001 & 0.155 & -0.765 & 0.048 & -0.822 & -0.060 & -5 \\
\hline 352.8 & 9.162 & 2000 & 1.575 & 0.001 & 0.148 & -0.774 & 0.065 & -0.881 & -0.052 & -5 \\
\hline 414.1 & 9.523 & 2000 & 1.706 & 0.001 & 0.154 & -0.853 & 0.065 & -0.952 & -0.063 & -5 \\
\hline 480.2 & 9.872 & 2000 & 1.837 & 0.002 & 0.159 & -0.928 & 0.063 & -1.013 & -0.062 & -5 \\
\hline 551.3 & 10.258 & 2000 & 1.969 & 0.002 & 0.168 & -1.029 & 0.068 & -1.085 & -0.059 & -5 \\
\hline 0.0 & 13.540 & 3000 & 0.000 & 0.001 & 0.280 & 0.000 & 0.000 & 0.000 & 0.000 & -5 \\
\hline 2.5 & 13.623 & 3000 & 0.087 & 0.001 & 0.272 & -0.097 & 0.070 & -0.125 & -0.121 & -5 \\
\hline 9.8 & 14.096 & 3000 & 0.175 & 0.001 & 0.260 & -0.193 & 0.120 & -0.236 & -0.261 & -5 \\
\hline 22.1 & 14.923 & 3000 & 0.262 & 0.001 & 0.259 & -0.290 & 0.079 & -0.354 & -0.370 & -5 \\
\hline 39.2 & 15.685 & 3000 & 0.350 & 0.001 & 0.257 & -0.337 & 0.091 & -0.468 & -0.406 & -5 \\
\hline 61.3 & 16.270 & 3000 & 0.437 & 0.001 & 0.252 & -0.366 & 0.108 & -0.583 & -0.357 & -5 \\
\hline 88.2 & 16.581 & 3000 & 0.525 & 0.001 & 0.243 & -0.399 & 0.109 & -0.695 & -0.261 & -5 \\
\hline 120.1 & 16.947 & 3000 & 0.612 & 0.001 & 0.233 & -0.452 & 0.094 & -0.812 & -0.176 & -5 \\
\hline 156.8 & 17.261 & 3000 & 0.700 & 0.001 & 0.228 & -0.516 & 0.066 & -0.926 & -0.120 & -5 \\
\hline 198.5 & 17.666 & 3000 & 0.787 & 0.001 & 0.226 & -0.584 & 0.034 & -1.036 & -0.069 & -5 \\
\hline 245.0 & 17.990 & 3000 & 0.875 & 0.001 & 0.223 & -0.652 & -0.002 & -1.152 & -0.033 & -5 \\
\hline 296.5 & 18.487 & 3000 & 0.962 & 0.001 & 0.224 & -0.738 & -0.021 & -1.276 & 0.004 & -5 \\
\hline 352.8 & 18.869 & 3000 & 1.050 & 0.001 & 0.223 & -0.815 & 0.057 & -1.392 & -0.008 & -5 \\
\hline 414.1 & 19.190 & 3000 & 1.137 & 0.001 & 0.223 & -0.898 & 0.074 & -1.493 & -0.025 & -5 \\
\hline 480.2 & 19.769 & 3000 & 1.225 & 0.001 & 0.221 & -0.966 & 0.066 & -1.618 & -0.050 & -5 \\
\hline 551.3 & 20.331 & 3000 & 1.312 & 0.001 & 0.225 & -1.046 & 0.071 & -1.743 & -0.062 & -5 \\
\hline 0.0 & 23.616 & 4000 & 0.000 & 0.001 & 0.492 & 0.000 & 0.000 & 0.000 & 0.000 & -5 \\
\hline 2.5 & 23.802 & 4000 & 0.066 & 0.001 & 0.483 & -0.130 & 0.076 & -0.153 & -0.202 & -5 \\
\hline 9.8 & 24.329 & 4000 & 0.131 & 0.001 & 0.465 & -0.250 & 0.158 & -0.306 & -0.388 & -5 \\
\hline 22.1 & 25.322 & 4000 & 0.197 & 0.001 & 0.447 & -0.379 & 0.213 & -0.461 & -0.560 & -5 \\
\hline 39.2 & 26.547 & 4000 & 0.262 & 0.001 & 0.422 & -0.459 & 0.320 & -0.620 & -0.685 & -5 \\
\hline 61.3 & 27.624 & 4000 & 0.328 & 0.001 & 0.397 & -0.537 & 0.455 & -0.754 & -0.739 & -5 \\
\hline 88.2 & 28.527 & 4000 & 0.394 & 0.001 & 0.382 & -0.555 & 0.501 & -0.919 & -0.694 & -5 \\
\hline 120.1 & 29.332 & 4000 & 0.459 & 0.001 & 0.366 & -0.602 & 0.531 & -1.069 & -0.627 & -5 \\
\hline 156.8 & 29.856 & 4000 & 0.525 & 0.001 & 0.353 & -0.671 & 0.504 & -1.227 & -0.522 & -5 \\
\hline 198.5 & 30.635 & 4000 & 0.591 & 0.001 & 0.352 & -0.735 & 0.441 & -1.386 & -0.428 & -5 \\
\hline 245.0 & 31.133 & 4000 & 0.656 & 0.001 & 0.348 & -0.776 & 0.380 & -1.521 & -0.357 & -5 \\
\hline 296.5 & 31.878 & 4000 & 0.722 & 0.001 & 0.342 & -0.851 & 0.333 & $\begin{array}{l}-1.702 \\
\end{array}$ & -0.308 & -5 \\
\hline 352.8 & 32.693 & 4000 & 0.787 & 0.001 & 0.340 & -0.907 & 0.295 & -1.874 & -0.262 & -5 \\
\hline 414.1 & 32.973 & 4000 & 0.853 & 0.001 & 0.333 & -0.989 & 0.238 & -2.016 & -0.207 & -5 \\
\hline 480.2 & 33.579 & 4000 & 0.919 & 0.001 & 0.328 & -1.052 & 0.198 & -2.183 & -0.193 & -5 \\
\hline 551.3 & 34.369 & 4000 & 0.984 & 0.001 & 0.330 & -1.148 & 0.145 & -2.369 & -0.116 & -5 \\
\hline
\end{tabular}


T-Motor 18x6.1 performance data table at $\theta=-15$ deg.

\begin{tabular}{|c|c|c|c|c|c|c|c|c|c|c|}
\hline $\mathrm{q}, \mathrm{Pa}$ & T_rho, $\mathrm{N}$ & $\mathrm{RPM}$ & $\mathrm{J}_{\infty}$ & $\mathrm{CP}$ & Q_rho, Nm & Fx_rho, N & Fy_rho, N & Mx_rho, Nm & My_rho, Nm & $\theta, \operatorname{deg}$ \\
\hline 0.0 & 5.532 & 2000 & 0.000 & 0.001 & 0.126 & 0.000 & 0.000 & 0.000 & 0.000 & -15 \\
\hline 2.5 & 5.899 & 2000 & 0.131 & 0.001 & 0.116 & -0.065 & 0.025 & -0.080 & -0.146 & -15 \\
\hline 9.8 & 6.319 & 2000 & 0.262 & 0.001 & 0.116 & -0.127 & 0.007 & -0.148 & -0.192 & -15 \\
\hline 22.1 & 6.379 & 2000 & 0.394 & 0.001 & 0.120 & -0.185 & -0.011 & -0.221 & -0.058 & -15 \\
\hline 39.2 & 6.499 & 2000 & 0.525 & 0.001 & 0.130 & -0.268 & -0.069 & -0.286 & 0.037 & -15 \\
\hline 61.3 & 6.731 & 2000 & 0.656 & 0.001 & 0.135 & -0.344 & 0.087 & -0.352 & -0.090 & -15 \\
\hline 88.2 & 6.628 & 2000 & 0.787 & 0.001 & 0.149 & -0.444 & 0.092 & -0.401 & -0.105 & -15 \\
\hline 120.1 & 6.518 & 2000 & 0.919 & 0.002 & 0.165 & -0.534 & 0.093 & -0.440 & -0.094 & -15 \\
\hline 156.8 & 6.305 & 2000 & 1.050 & 0.002 & 0.185 & $\begin{array}{l}-0.637 \\
\end{array}$ & 0.092 & -0.465 & -0.074 & -15 \\
\hline 198.5 & 6.292 & 2000 & 1.181 & 0.002 & 0.204 & -0.748 & 0.092 & -0.487 & -0.048 & -15 \\
\hline 245.0 & 6.373 & 2000 & 1.312 & 0.002 & 0.221 & -0.843 & 0.087 & -0.508 & -0.028 & -15 \\
\hline 296.5 & 6.578 & 2000 & 1.444 & 0.002 & 0.237 & -0.940 & 0.084 & -0.533 & -0.017 & -15 \\
\hline 352.8 & 6.824 & 2000 & 1.575 & 0.002 & 0.239 & -0.967 & 0.090 & -0.557 & -0.009 & -15 \\
\hline 414.1 & 7.105 & 2000 & 1.706 & 0.002 & 0.263 & -1.132 & 0.081 & -0.574 & -0.001 & -15 \\
\hline 480.2 & 7.445 & 2000 & 1.837 & 0.003 & 0.276 & -1.234 & 0.067 & -0.603 & 0.012 & -15 \\
\hline 551.3 & 7.947 & 2000 & 1.969 & 0.003 & 0.289 & -1.349 & 0.056 & -0.652 & 0.015 & -15 \\
\hline 0.0 & 13.540 & 3000 & 0.000 & 0.001 & 0.280 & 0.000 & 0.000 & 0.000 & 0.000 & -15 \\
\hline 2.5 & 13.789 & 3000 & 0.087 & 0.001 & 0.270 & -0.091 & 0.076 & -0.119 & -0.122 & -15 \\
\hline 9.8 & 14.506 & 3000 & 0.175 & 0.001 & 0.256 & -0.187 & 0.134 & -0.234 & -0.288 & -15 \\
\hline 22.1 & 15.424 & 3000 & 0.262 & 0.001 & 0.265 & -0.275 & 0.060 & -0.356 & -0.418 & -15 \\
\hline 39.2 & 15.456 & 3000 & 0.350 & 0.001 & 0.247 & -0.263 & 0.114 & -0.449 & -0.293 & -15 \\
\hline 61.3 & 15.384 & 3000 & 0.437 & 0.001 & 0.250 & -0.320 & 0.012 & -0.564 & -0.081 & -15 \\
\hline 88.2 & 15.482 & 3000 & 0.525 & 0.001 & 0.250 & -0.384 & -0.092 & -0.681 & 0.064 & -15 \\
\hline 120.1 & 15.822 & 3000 & 0.612 & 0.001 & 0.246 & -0.444 & 0.153 & -0.796 & -0.130 & -15 \\
\hline 156.8 & 16.130 & 3000 & 0.700 & 0.001 & 0.245 & -0.519 & 0.202 & -0.904 & -0.189 & -15 \\
\hline 198.5 & 16.146 & 3000 & 0.787 & 0.001 & 0.250 & -0.598 & 0.214 & -0.991 & -0.222 & -15 \\
\hline 245.0 & 16.213 & 3000 & 0.875 & 0.001 & 0.262 & $\begin{array}{l}-0.697 \\
\end{array}$ & 0.210 & $\begin{array}{l}-1.092 \\
\end{array}$ & -0.235 & -15 \\
\hline 296.5 & 16.251 & 3000 & 0.962 & 0.001 & 0.277 & -0.795 & 0.191 & -1.184 & -0.235 & -15 \\
\hline 352.8 & 15.970 & 3000 & 1.050 & 0.001 & 0.308 & -0.950 & 0.180 & -1.228 & -0.229 & -15 \\
\hline 414.1 & 16.132 & 3000 & 1.137 & 0.001 & 0.318 & -1.027 & 0.158 & -1.310 & -0.200 & -15 \\
\hline 480.2 & 16.260 & 3000 & 1.225 & 0.001 & 0.324 & -1.111 & 0.191 & -1.363 & -0.161 & -15 \\
\hline 551.3 & 16.271 & 3000 & 1.312 & 0.001 & 0.345 & -1.242 & 0.196 & -1.392 & -0.123 & -15 \\
\hline 0.0 & 23.616 & 4000 & 0.000 & 0.001 & 0.492 & 0.000 & 0.000 & 0.000 & 0.000 & -15 \\
\hline 2.5 & 24.048 & 4000 & 0.066 & 0.001 & 0.481 & -0.120 & 0.087 & -0.150 & -0.225 & -15 \\
\hline 9.8 & 24.823 & 4000 & 0.131 & 0.001 & 0.460 & -0.245 & 0.174 & -0.298 & -0.409 & -15 \\
\hline 22.1 & 26.084 & 4000 & 0.197 & 0.001 & 0.432 & -0.350 & 0.274 & -0.451 & -0.626 & -15 \\
\hline 39.2 & 27.377 & 4000 & 0.262 & 0.001 & 0.393 & -0.410 & 0.462 & -0.620 & -0.765 & -15 \\
\hline 61.3 & 27.872 & 4000 & 0.328 & 0.001 & 0.359 & -0.436 & 0.567 & -0.740 & -0.699 & -15 \\
\hline 88.2 & 28.243 & 4000 & 0.394 & 0.001 & 0.350 & -0.480 & 0.474 & -0.895 & -0.495 & -15 \\
\hline 120.1 & 28.351 & 4000 & 0.459 & 0.001 & 0.355 & -0.514 & 0.311 & -1.056 & -0.238 & -15 \\
\hline 156.8 & 28.687 & 4000 & 0.525 & 0.001 & 0.349 & -0.573 & 0.212 & -1.226 & -0.090 & -15 \\
\hline 198.5 & 29.100 & 4000 & 0.591 & 0.001 & 0.339 & -0.601 & -0.104 & -1.395 & -0.068 & -15 \\
\hline 245.0 & 29.667 & 4000 & 0.656 & 0.001 & 0.335 & -0.666 & 0.026 & -1.571 & -0.193 & -15 \\
\hline 296.5 & 29.859 & 4000 & 0.722 & 0.001 & 0.323 & -0.737 & 0.088 & -1.698 & -0.263 & -15 \\
\hline 352.8 & 30.564 & 4000 & 0.787 & 0.001 & 0.326 & -0.829 & 0.169 & -1.905 & -0.314 & -15 \\
\hline 414.1 & 30.612 & 4000 & 0.853 & 0.001 & 0.320 & -0.933 & 0.215 & -2.064 & -0.357 & -15 \\
\hline 480.2 & 31.107 & 4000 & 0.919 & 0.001 & 0.334 & -1.015 & 0.284 & -2.247 & -0.367 & -15 \\
\hline 551.3 & 31.363 & 4000 & 0.984 & 0.001 & 0.342 & -1.099 & 0.280 & -2.386 & -0.387 & -15 \\
\hline
\end{tabular}


T-Motor 18x6.1 performance data table at $\theta=-90 \mathrm{deg}$.

\begin{tabular}{|c|c|c|c|c|c|c|c|c|c|c|}
\hline $\mathrm{q}, \mathrm{Pa}$ & T_rho, $\mathrm{N}$ & $\mathrm{RPM}$ & $\mathrm{J}_{\infty}$ & $\mathrm{CP}$ & Q_rho, Nm & Fx_rho, N & Fy_rho, N & Mx_rho, Nm & My_rho, Nm & $\theta, \operatorname{deg}$ \\
\hline 0.0 & 5.532 & 2000 & 0.000 & 0.001 & 0.126 & 0.000 & 0.000 & 0.000 & 0.000 & -90 \\
\hline 2.5 & 6.354 & 2000 & 0.131 & 0.001 & 0.120 & 0.000 & 0.000 & 0.000 & 0.000 & -90 \\
\hline 9.8 & 6.850 & 2000 & 0.262 & 0.001 & 0.113 & 0.000 & 0.000 & 0.000 & 0.000 & -90 \\
\hline 22.1 & 7.091 & 2000 & 0.394 & 0.001 & 0.108 & 0.000 & 0.000 & 0.000 & 0.000 & -90 \\
\hline 39.2 & 5.469 & 2000 & 0.525 & 0.001 & 0.145 & 0.000 & 0.000 & 0.000 & 0.000 & -90 \\
\hline 61.3 & 5.985 & 2000 & 0.656 & 0.001 & 0.134 & 0.000 & 0.000 & 0.000 & 0.000 & $\begin{array}{l}-90 \\
\end{array}$ \\
\hline 88.2 & 6.359 & 2000 & 0.787 & 0.001 & 0.131 & 0.000 & 0.000 & 0.000 & 0.000 & -90 \\
\hline 120.1 & 6.463 & 2000 & 0.919 & 0.001 & 0.132 & 0.000 & 0.000 & 0.000 & 0.000 & $\begin{array}{l}-90 \\
\end{array}$ \\
\hline 156.8 & 6.526 & 2000 & 1.050 & 0.001 & 0.133 & 0.000 & 0.000 & 0.000 & 0.000 & $\begin{array}{c}-90 \\
\end{array}$ \\
\hline 198.5 & 7.494 & 2000 & 1.181 & 0.001 & 0.102 & 0.000 & 0.000 & 0.000 & 0.000 & -90 \\
\hline 245.0 & 11.538 & 2000 & 1.312 & 0.006 & 0.647 & 0.000 & 0.000 & 0.000 & 0.000 & -90 \\
\hline 296.5 & 13.019 & 2000 & 1.444 & 0.007 & 0.715 & 0.000 & 0.000 & 0.000 & 0.000 & -90 \\
\hline 352.8 & 14.608 & 2000 & 1.575 & 0.007 & 0.784 & 0.000 & 0.000 & 0.000 & 0.000 & -90 \\
\hline 414.1 & 16.309 & 2000 & 1.706 & 0.008 & 0.855 & 0.000 & 0.000 & 0.000 & 0.000 & -90 \\
\hline 480.2 & 18.122 & 2000 & 1.837 & 0.009 & 0.928 & 0.000 & 0.000 & 0.000 & 0.000 & -90 \\
\hline 551.3 & 20.049 & 2000 & 1.969 & 0.010 & 1.002 & 0.000 & 0.000 & 0.000 & 0.000 & -90 \\
\hline 0.0 & 13.540 & 3000 & 0.000 & 0.001 & 0.280 & 0.000 & 0.000 & 0.000 & 0.000 & -90 \\
\hline 2.5 & 14.369 & 3000 & 0.087 & 0.001 & 0.265 & 0.000 & 0.000 & 0.000 & 0.000 & -90 \\
\hline 9.8 & 14.947 & 3000 & 0.175 & 0.001 & 0.252 & 0.000 & 0.000 & 0.000 & 0.000 & -90 \\
\hline 22.1 & 15.561 & 3000 & 0.262 & 0.001 & 0.245 & 0.000 & 0.000 & 0.000 & 0.000 & -90 \\
\hline 39.2 & 15.968 & 3000 & 0.350 & 0.001 & 0.235 & 0.000 & 0.000 & 0.000 & 0.000 & -90 \\
\hline 61.3 & 16.274 & 3000 & 0.437 & 0.001 & 0.232 & 0.000 & 0.000 & 0.000 & 0.000 & -90 \\
\hline 88.2 & 12.792 & 3000 & 0.525 & 0.001 & 0.332 & 0.000 & 0.000 & 0.000 & 0.000 & -90 \\
\hline 120.1 & 14.031 & 3000 & 0.612 & 0.001 & 0.308 & 0.000 & 0.000 & 0.000 & 0.000 & -90 \\
\hline 156.8 & 14.761 & 3000 & 0.700 & 0.001 & 0.299 & 0.000 & 0.000 & 0.000 & 0.000 & $\begin{array}{l}-90 \\
\end{array}$ \\
\hline 198.5 & 15.269 & 3000 & 0.787 & 0.001 & 0.296 & 0.000 & 0.000 & 0.000 & 0.000 & $\begin{array}{l}-90 \\
\end{array}$ \\
\hline 245.0 & 15.898 & 3000 & 0.875 & 0.001 & 0.293 & 0.000 & 0.000 & 0.000 & 0.000 & $\begin{array}{l}-90 \\
\end{array}$ \\
\hline 296.5 & 16.165 & 3000 & 0.962 & 0.001 & 0.294 & 0.000 & 0.000 & 0.000 & 0.000 & -90 \\
\hline 352.8 & 16.349 & 3000 & 1.050 & 0.001 & 0.296 & 0.000 & 0.000 & 0.000 & 0.000 & -90 \\
\hline 414.1 & 17.397 & 3000 & 1.137 & 0.001 & 0.229 & 0.000 & 0.000 & 0.000 & 0.000 & -90 \\
\hline 480.2 & 16.511 & 3000 & 1.225 & 0.001 & 0.300 & 0.000 & 0.000 & 0.000 & 0.000 & -90 \\
\hline 551.3 & 26.527 & 3000 & 1.312 & 0.006 & 1.397 & 0.000 & 0.000 & 0.000 & 0.000 & -90 \\
\hline 0.0 & 23.616 & 4000 & 0.000 & 0.001 & 0.492 & 0.000 & 0.000 & 0.000 & 0.000 & -90 \\
\hline 2.5 & 25.003 & 4000 & 0.066 & 0.001 & 0.477 & 0.000 & 0.000 & 0.000 & 0.000 & -90 \\
\hline 9.8 & 26.027 & 4000 & 0.131 & 0.001 & 0.460 & 0.000 & 0.000 & 0.000 & 0.000 & -90 \\
\hline 22.1 & 26.936 & 4000 & 0.197 & 0.001 & 0.444 & 0.000 & 0.000 & 0.000 & 0.000 & -90 \\
\hline 39.2 & 27.795 & 4000 & 0.262 & 0.001 & 0.437 & 0.000 & 0.000 & 0.000 & 0.000 & $\begin{array}{l}-90 \\
\end{array}$ \\
\hline 61.3 & 28.372 & 4000 & 0.328 & 0.001 & 0.424 & 0.000 & 0.000 & 0.000 & 0.000 & -90 \\
\hline 88.2 & 28.895 & 4000 & 0.394 & 0.001 & 0.424 & 0.000 & 0.000 & 0.000 & 0.000 & -90 \\
\hline 120.1 & 29.377 & 4000 & 0.459 & 0.001 & 0.410 & 0.000 & 0.000 & 0.000 & 0.000 & -90 \\
\hline 156.8 & 25.099 & 4000 & 0.525 & 0.001 & 0.274 & 0.000 & 0.000 & 0.000 & 0.000 & -90 \\
\hline 198.5 & 25.377 & 4000 & 0.591 & 0.001 & 0.287 & 0.000 & 0.000 & 0.000 & 0.000 & -90 \\
\hline 245.0 & 27.441 & 4000 & 0.656 & 0.001 & 0.283 & 0.000 & 0.000 & 0.000 & 0.000 & -90 \\
\hline 296.5 & 28.409 & 4000 & 0.722 & 0.001 & 0.293 & 0.000 & 0.000 & 0.000 & 0.000 & $\begin{array}{l}-90 \\
\end{array}$ \\
\hline 352.8 & 28.585 & 4000 & 0.787 & 0.001 & 0.286 & 0.000 & 0.000 & 0.000 & 0.000 & $\begin{array}{l}-90 \\
\end{array}$ \\
\hline 414.1 & 29.925 & 4000 & 0.853 & 0.001 & 0.268 & 0.000 & 0.000 & 0.000 & 0.000 & $\begin{array}{c}-90 \\
\end{array}$ \\
\hline 480.2 & 30.021 & 4000 & 0.919 & 0.001 & 0.264 & 0.000 & 0.000 & 0.000 & 0.000 & $\begin{array}{l}-90 \\
\end{array}$ \\
\hline 551.3 & 30.155 & 4000 & 0.984 & 0.001 & 0.264 & 0.000 & 0.000 & 0.000 & 0.000 & -90 \\
\hline
\end{tabular}


APPENDIX 


\section{References}

[1] S. Gupte, P. I. T. Mohandas, and J. M. Conrad, "A survey of quadrotor unmanned aerial vehicles," Conference Proceedings - IEEE SOUTHEASTCON, 2012.

[2] Aeryon Labs Inc, "Aeryon skyranger the benchmark for vtol suas," accessed 04 June 2017. [Online]. Available: https://www.aeryon.com/aeryon-skyranger

[3] DJI, "Spreading wings s1000," accessed 20 November 2017. [Online]. Available: https: //www.dji.com/spreading-wings-s1000

[4] C. Powers, D. Mellinger, A. Kushleyev, B. Kothmann, and V. Kumar, "Influence of Aerodynamics and Proximity Effects in Quadrotor Flight," Springer Tracts in Advanced Robotics - Experimental Robotics, vol. 88, pp. 289-302, 2013.

[5] G. M. Hoffmann, S. L. Waslander, and C. J. Tomlin, "Aerodynamics and control of autonomous quadrotor helicopters in aggressive maneuvering," 2009.

[6] W. Khan and M. Nahon, "Toward an accurate physics-based uav thruster model," IEEE/ASME Transactions on Mechatronics, vol. 18, no. 4, pp. 1269-1279, 2013.

[7] B. Theys, G. Dimitriadis, P. Hendrick, and J. De Schutter, "Experimental and numerical study of micro-aerial-vehicle propeller performance in oblique flow," Journal of Aircraft, pp. 1-9, oct 2016.

[8] T. B. Carroll, I.-R. George, G. Bramesfeld, and K. Raahemifar, "Design optimization of small rotors in quad-rotor configuration," 54th AIAA Aerospace Sciences Meeting, no. January, pp. 1-17, 2016.

[9] J. Luo, L. Zhu, and G. Yan, "Novel quadrotor forward-flight model based on wake interference," AIAA Journal, vol. 53, no. 12, pp. 3522-3533, 2015. 
[10] P. Ventura Diaz and S. Yoon, "High-Fidelity Computational Aerodynamics of Multi-Rotor Unmanned Aerial Vehicles," 2018 AIAA Aerospace Sciences Meeting, no. January, pp. 1-22, 2018.

[11] R. C. Dingeldein, "Wind-Tunnel Studies of the Performance of Multirotor Configuration," National Advisory Committee for Aeronautics, Tech. Rep. 3236, 1954.

[12] J. De Leeuw and W. Castles, "The normal component of the induced velocity in the vicinity of a lifting rotor and some examples of its application," National Advisory Committee for Aeronautics, Tech. Rep. 1184, 1955.

[13] H. H. Heyson, "Induced Flow Near a Helicopter Rotor: A Review of Present Knowledge." American Helicopter Society, 1959.

[14] I.-R. E. George, "A multirotor vehicle performance prediction method," Master's thesis, Ryerson University, 2017.

[15] W. Castles and H. Durham, "Distribution of Normal Component of Induced Velocity in Lateral Plane of a Lifting Rotor," Tech. Rep. 3841, 1956.

[16] J. G. Leishman, Principles of Helicopter Aerodynamics, 2nd ed. Cambridge University Press, 2006.

[17] T. B. Carroll, "A design methodology for rotors of small multirotor vehicles," Master's thesis, Ryerson University, 2017.

[18] H. H. Heyson and S. Katzoff, "Normal Component of Induced Velocity in the Vicinity of a Lifting Rotor with a Nonuniform Disk Loading," National Advisory Committee for Aeronautics, Langley Field, Va., Tech. Rep. April, 1956.

[19] J. Katz and A. Plotkin, Low-Speed Aerodynamics. Cambridge University Press, 2001.

[20] C. Russell, J. Jung, G. Willinuk, and B. Glasner, "Wind tunnel and hover performance test results for multicopter uas vehicles." AHS 72nd Annual Forum, 2016.

[21] T. Martin, "Testing and Improvements of a Bluff Body Designed for a Small Unmanned Aerial Multirotor Vehicle," Undergraduate Thesis, Ryerson University, 2017.

[22] B. W. McCormick, Aerodynamics, aeronautics, and flight mechanics. Wiley New York, 1995, vol. 2. 
[23] M. Ramasamy, J. G. Leishman, and T. E. Lee, "Flowfield of a Rotating-Wing Micro Air Vehicle," Journal of Aircraft, vol. 44, no. 4, pp. 1236-1244, jul 2007.

[24] Photoking, "3DR Solo - The Smart Drone." [Online]. Available: http://videoking.cz/shop/ multikoptery/3dr-solo/

[25] J. Martin, "3DR Solo review," accessed 06 June 2017. [Online]. Available: https: //www.techadvisor.co.uk/review/drones/3dr-solo-review-uk-3624759/

[26] J. Brandt, "Small scale propeller performance at low speeds," Master's thesis, 2005.

[27] Amazon, "EJH Precision Prop Balancer 3D Robotics 3DR Solo," accessed 12 January 2017. [Online]. Available: https://www.amazon.com/gp/product/B0108LIS4K/ref=as $\{-\}$ li $\{-\}$ tl ie $=$ UTF $8\{\&\}$ tag $=$ thebasicpilot $20\{\&\}$ camp $=1789\{\&\}$ creative $=9325\{\&\} \operatorname{linkCode}=$ as $2\{\&\}$ creativeASIN=B0108LIS4K $\{\&\}$ linkId=43f903b64716279c7cad6895ff07bdf4

[28] J. Brandt, R. Deters, G. Ananda, and M. Selig, "UIUC Propeller Databse," accessed 01 November 2017. [Online]. Available: http://m-selig.ae.illinois.edu/props/propDB.html

[29] R. Niemiec and F. Gandhi, "Multirotor Controls, Trim, and Autonomous Flight Dynamics of Plusand Cross-Quadcopters," Journal of Aircraft, vol. 54, no. 5, pp. 1910-1920, 2017.

[30] D. F. Barcelos, "Aerodynamic Analysis of Multirotor Vehicles Using a Higher-Order Potential Flow Method by," Master's thesis, Ryerson University, 2018. 
